\title{
A POLARIZED PHOTOLUMINESCENCE STUDY OF STRAINED LAYER GAAS PHOTOCATHODES
}

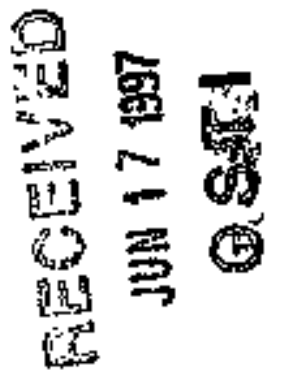

\section{DISCLAIMER}

This tepert was prepared as an acoount of work spossored by an neency of the Unitad Stutes Goweromeat. Nefither the United States Government nor any agency thereof, nor any of their

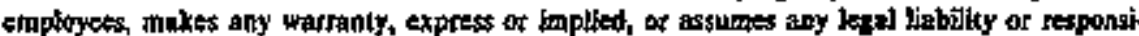

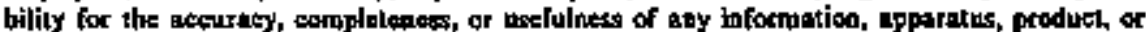
process disclosed, of repsesents that its ust would ank fifinge privglely ontned rights. Reforonce herein to any specifie oommerciat product, process, or service by trade name, tradematk. menufaclurer, or otherevise does not necesserily contstilute or imply hs endortecotonl, recorrmendation, or faporiog by the United states Government or taly agency theteof. The vitus and apinions of authors expressed bereth do nol acocassarly stato or teflect those of the Unthed States Goveramemt or any agency thereof.

\author{
Robin Alexander Mair
}

SLAC-Report-488

July 1996

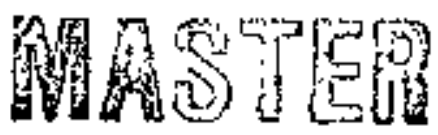

DISTRIBUTION OF THIS DOCUMENTIS UNHIMIH Prepared for the Department of Energy under contract number DE-AC03-76SF00515 
This document and the material and data contajned therein, was devel. oped under sponsorship of the United Stales Government. Neither the United Stutes nor the Department of Energy, nor the Leland Stanford Junior University, nos their employees, nor their respective contractors, subcontractors, or their employees, makes any warranty, express or implied, or assumes any liability or responsibility for accuracy, complete. ness or usefulness of any information, apparatus, product or process disclosed, or represents that jts use will not infringe privalely-owned rights. Mention of any product, its manufacturer, or suppliers shall not, nor is it intended to, imply approval, disapproval, or fitness for any particular use. A royalty-free, nonexclusive right to use and disseminate same for any purpose whatsoever, is expressly reserved to the United States and the University. 


\section{DISCLADMIXR}

Portions of this doctrment may be illegible in electronic image produets. Images are produced from the best svailable original document. 
SLAC-R-488

WISC-EX-96-346

UC-414

\title{
A POLARIZED PHOTOLUMINESCENCE STUDY OF STRAINED LAYER GaAs PHOTOCATHODES*
}

\author{
Robin Alexander Mair \\ Stanford Linear Accelerator Center \\ Stanford University \\ Stanford, California 94309
}

SLAC-Report-488

July 1996

Prepared for the Department of Energy under contract numbers

DE-AC03-76SF00515 (SLAC) and DE-AC02-76ER00881 (UW).

Printed in the United States of America. Available from the National Technical Information Service, U.S. Department of Commerce, 5285 Port Royal Road, Springtield, Virginia 22161.

Ph.D. thesis, University of Wisconsin, Madison WI 53706 


\begin{abstract}
Photoluminescence measurements bave been made on a set of epitaxially grown strained GaAs photocathode structures. The photocathodes are designed to exhibit a strain-induced enbancement of the electron spin polarization obtainable by optical pumping with circularly polarized radiation of near band gap energy. For the case of non-strajned GaAs, the degree of spin polarization is limited to $50 \%$ by crystal symmetry. Under an appropriate uniaxial compression or tension, however, the valence band stracture near the gap minimum is modified such that a spin polarization of $100 \%$ is theoretically possible.

A total of nine samples with biaxial compressive strains ranging from zero to $\sim 0.8 \%$ are studied. $\mathrm{X}$-ray diffraction analysis, utilizing Bragg reflections, is used to determine the crystal fattjce structure of the samples. Luninescence spectra and luminescence circular polarization data are obtained at room temperature, $\approx 78 \mathrm{~K}$ and $\approx 12 \mathrm{~K}$. The degree of luminescence circular polarization is used as a relative measure of the photo-excited electron spin polarization. The room temperature luminescence circular polarization data is compared with the measured electron spin polarization when the samples are used as electron photo-emitters with a negative electron affinity surface preparation.

The luminescence data is also analyzed in conjunction with the crystal structure data with the goal of understanding the strain dependent valence band structure, optical purmping characteristics and spin depolarization mechanisms of the photocathode structures. A simple model is used to describe the luminescence data obtained for the set of samples. Within the assumptions of the model, the defor-
\end{abstract}


mation potentials $a, b$ and $d$ for GaAs are determined. The measured values are $a=-10.16 \pm .21 \mathrm{eV}, b=-2.00 \pm .05 \mathrm{eV}$ and $d=-4.87 \pm .29 \mathrm{eV}$. Good agreement with published values of the deformation potentials provides support for the model used to describe the data. Comparison of the luminescence circular polarization data for the various samples and temperatures is interpreted in terms of a spin depolarization process which occurs at the surface or in the surface band bending region of the samples. 


\section{Acknowledgements}

The experimental studies presented in this dissertation were performed at the Stanford Linear Accelerator Center (SLAC) as part of an active research and development program maintained by a SLAC/Wisconsin collaboration. I am grateful to my advisor, Dick Prepost, for providing me with the opportunity to participate in this program. The key members of the SLAC/Wisconsin collaboration, Dr. Ed Garwin of SLAC, Dr. Takashi Maruyana of SLAC and Professor Richard Prepost of the University of Wisconsin-Madison, were each valuable sources of knowledge, advice and encouragement.

I am additionally grateful to Ed Garwin for the facilities and support made available during my stay at SLAC. I wish to thank the people of the Physical Electronics Group: Jerry Collet, Jean Francis, Dr. Ed Garwin, Frank King, Dr. Bob Kirby, Joe Praxel, Lee Shere and Ladia Turek. In addition to the various technical support and advice, the camaraderie shared with these indivjduals is truly appreciated. The good coffee, popcorn, pizza Junches and conversations at the picnic table will be missed.

The collaborative efforts and frujtful conversations shared with Dr. Huan Tang and Dr. Gregory Mulhollan are genuinely appreciated. I both enjoyed and benefitted from my interaction with these individuals and several other Accelerator Department members involved with electron sources and source polarization at SLAC.

I gratefully acknowledge the financial support provided by SLAC during the majority of my stay, and I thank Dr. David Leith and Dr. Ed Garwin for arranging 
this support.

I would like to extend ar additional expression of gratitude to Bob Kirby and Takashj Maruyama for their assistance in acquiring some critjcal jnformatjon needed daring the writing of this dissertation.

I wish to thank Professors Ludwig Bruch, Willie Haeberli, Richard Matyi, Marshall Onellion and Richard Prepost for their participation on my dissertation defense committee.

The support and encouragement given by my friends and family, particulariy my mother and father, throughout my graduate career are greatly appreciated. It is a great comfort to know there is always an old and familiar place to call home.

Lastly, I thank my wife Christine for her constant support and enduring patience during trying times. I couldn't ask for a greater inspiration. 


\section{Contents}

Abstract

1 Introduction 1

2 Band Structure of GaAs 10

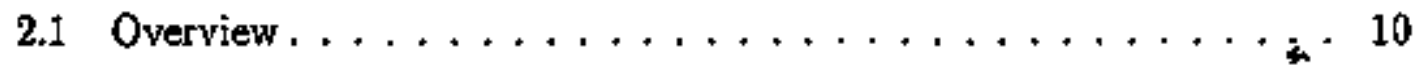

2.2 Band Structure of GaAs $\ldots \ldots \ldots \ldots$. . . . . . . . . . .

2.2 .1 Electrons in a Crystal Lattice . . . . . . . . . . . . 11

2.2 .2 The $k \cdot p$ Method . . . . . . . . . . . 13

$2.2 .3 k \cdot p$ Matrix for GaAs . . . . . . . . . . . 14

2.2.4 Spin Orbit Interaction . . . . . . . . . . . 16

2.3 Strain Induced Effects near $\mathbf{I} \ldots \ldots \ldots$

2.3 .1 Strajo Interaction Matrix . . . . . . . . . . . . 20

2.3.2 Biaxial Compression .................. 22

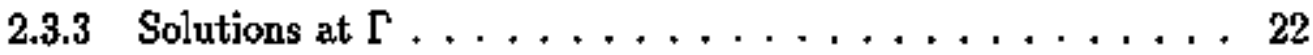

3 Optical Transitions and Spin Polarization 26

3.1 Overview of Transition Probabilities . . . . . . . . . . . . 26

3.2 Spin Polarization from Optical Pumping . . . . . . . . . . 27

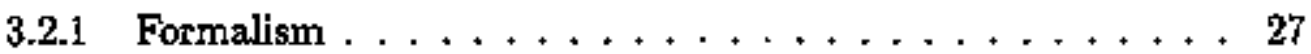


3.2.2 Optical Transitions to Conduction Band . . . . . . 29

3.3 Luminescence Circular Polarization $\ldots \ldots \ldots \ldots \ldots \ldots \ldots$

3.3.1 Description . . . . . . . . . . . . . 33

3.3 .2 Calculation $\ldots \ldots \ldots \ldots \ldots \ldots \ldots \ldots \ldots$

3.3.3 Spin Relaxation . . . . . . . . . . . . 37

3.3 .4 Final Expressions $\ldots \ldots \ldots \ldots \ldots \ldots$

3.4 Consideration of Non-Zero $\vec{k}$ Effects on Polarization $\ldots \ldots \ldots . .40$

4 Experimental Description 44

4.1 System Overview . . . . . . . . . . . . . 44

4.2 Photocathode Description $\ldots \ldots \ldots \ldots \ldots \ldots \ldots$

4.3 Sample Preparation . . . . . . . . . . . . . . . . . 49

4.3.1 General Preparation . . . . . . . . . . . . . . 49

4.3.2 Preparation for Luminescence Experiments . . . . . . . . 31

4.4 Cryostat and Sample Stage . . . . . . . . . . . . . 61

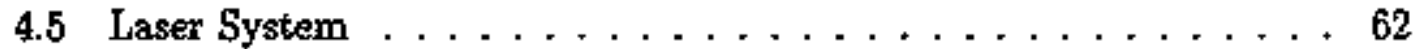

4.6 Optical System $\ldots \ldots \ldots \ldots \ldots \ldots \ldots \ldots$

4.7 Double Spectrometer . . . . . . . . . . . . . . . . 64

4.8 Detector and Data Acquisition System . . . . . . . . 67

4.9 System Calibrations $\ldots \ldots \ldots \ldots \ldots \ldots \ldots \ldots$

4.9 .1 Wave Plate Calibration . . . . . . . . . . . . 68

4.9.2 Spectrometer Wavelength Scale Calibration . . . . . . 69

4.9 .3 Spectral Response. . . . . . . . . . . . . . . 69

4.9.4 Laser Wavelength Calibration . . . . . . . . 70 
4.10 Luminescence Data Acquisition Methods . . . . . . . . . . 70

4.11 X-ray Diffraction Measurement of Lattice Strain . . . . . . . 72

$\begin{array}{ll}5 \text { Data and Discussion } & 76\end{array}$

5.1 X-Ray Diffraction Measurements $\ldots \ldots \ldots \ldots \ldots \ldots$

5.2 Luminescence $\operatorname{Spectra} \ldots \ldots \ldots \ldots \ldots \ldots$

5.2.1 Determination of Phosphorus Content in $\mathrm{GaAs}_{(1-x)} \mathrm{P}_{x} \ldots .85$

$5.2,2$ Determination of Epilayer Strain $\ldots \ldots \ldots 8$

5.2.3 Line-Shape of Luminescence Spectra . . . . . . . . . 95

5.3 Circular Polarization of Active Layer Luminescence $\ldots \ldots \ldots \ldots$

5.3.1 Dependence on Luminescence Wavelength . . . . . . 96

5.3.2 Dependence on Pump Wavelength . . . . . . . . . 107

5.4 Comparison of Luminescence Circular Polarization with Photo-Enitted Electron Spin Polarization . . . . . . . . . . . . . . 111

5.5 Luminescence Measurements at Low Temperatures . . . . . . . 131

5.5 .1 Measurements at $78 \mathrm{~K} \ldots \ldots \ldots \ldots \ldots$

5.5.2 Measurements at $12 \mathrm{~K} \ldots \ldots \ldots \ldots \ldots \ldots$

6 Analysis and Conclusions $\quad 155$

6.1 Deconvolution of Luminescence Spectra . . . . . . . . . . 158

6.2 Calculation of Deformation Potentials for GaAs . . . . . . . 162

6.2.1 Deformation Potential $b \ldots \ldots \ldots \ldots \ldots$

6.2.2 Deformation Potential a . . . . . . . . . . 164

6.2.3 Deformation Potential $d \ldots \ldots \ldots \ldots \ldots$ 
6.3 Characteristics of the Spin Relaxation Factor $R \ldots \ldots 7$

6.4 Conclusions $\ldots \ldots \ldots \ldots \ldots \ldots$

6.4.1 Possible Improvements to Photocathode Structure . . . . . . 177

A Calculation of Electron Spin Polarization Using Cubic Symmetryt80

B Remarks About Non-Thimned Strained Layer Sample \#9 183

C In-Plane Shear Strain and Linearly Polarized Luminescence Asymmetry

C.1 Measurement of In-Plane Shear . . . . . . . . . . . . 188

C.2 Linearly Polarized Luminescence Asymmetry . . . . . . . . . . . . 189

Bibliography 


\section{Chapter 1}

\section{Introduction}

The spin polarization of an ensemble of electrons may be defined as

$$
P_{e-}=\frac{N_{\uparrow}-N_{\downarrow}}{N_{\uparrow}+N_{\downarrow}}
$$

where $N_{\uparrow}\left(N_{\downarrow}\right)$ is the number of electrons with spin parallel (anti-parallel) to the direction along which the polarization is defined. A spin polarized electron beam offers the passibility of examining the spin dependent nature of various electron scattering processes.[1]

One method of producing a beam of polarized electrons is through the photoemission of spin polarized electrons from a semiconductor. When a direct band gap semiconductor of appropriate crystal symmetry is optically pumped with circularly polarized light, there is preferential pumping to one spin state leading to a net spin polarization among the photo-excited electrons. For electrons to escape from the semiconductor, the surface must be made to have negative electron affinity (NEA). A NEA surface is defined as a surface at which the vacuum level lies below the conduction band minimum within the semiconductor.[2] A diagram of a NEA surface is shown in Figure 1.1. Such a surface is created by deposition of a partial mono-layer of an alkali metal and either oxygen or fluorine onto the atomically 


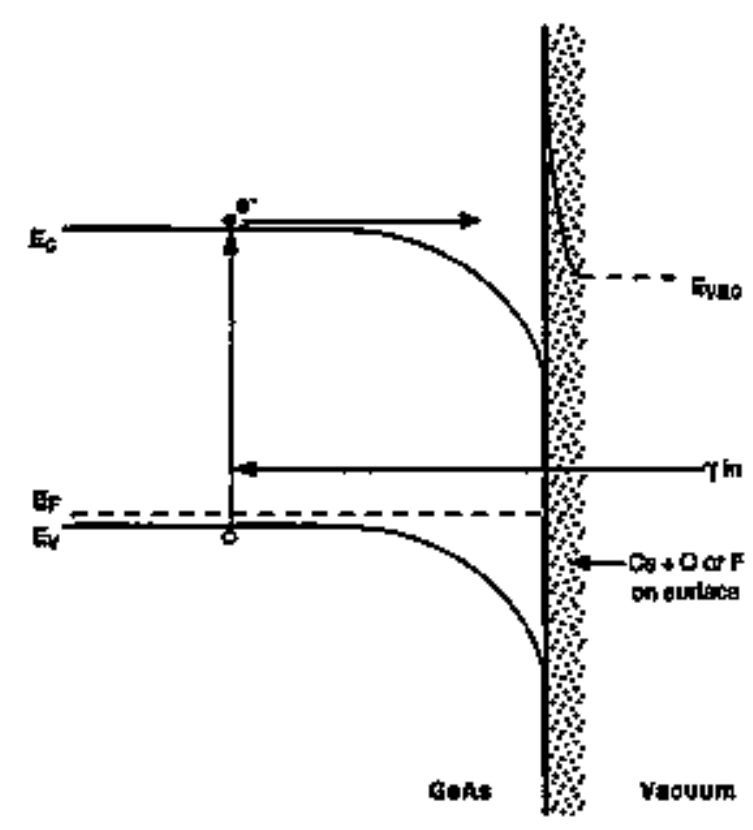

Figure 1.1: Diagram of an NEA surface showing band bending near the GaAs surface and lowering of the vacuum level.

clean semiconductor surface in an ultra-high vacuum environment. A bigh level of p-type doping places the Fermi level near the valence band maximum and provides enough free carriers to create a depletion region at the surface which is thin enough for conduction electrons to traverse without significant thermalization. The layer deposited on the surface of the semiconductor acts to bend the bands and lower the vacuum level relative to the Fermi level. If the band gap of the semiconductor is sufficiently large, the vacuam level can be lowered to a point below the conduction band minimum and the surface is said to be NEA.

Semiconductor candidates for a polarized electron source must meet the requirements described for both polarized electron production and successful activation to a state of negative electron affinity. The direct band gap members of the III.V family of semiconductors meet the requirements for polarized electron pro- 
duction, possessing the zinc-blende crystal symmetry and a corresponding band structure which allows for a theoretical spin polarization of $50 \%$. The requirements of high minority carrier mobility for electron transport to the cathode surface and sufficiently large band gap for NEA activation furtber limit the number of potential candidates. GaAs is a specific example of a III-V semiconductor which bas been used quite successfully as a polarized electron photocathode material and is the focus of the present study.

The GaAs spin polarized electron source has many attractive properties. First, the theoretical $50 \%$ polarizatjon limit has been approached in practice by designing cathodes with sufficiently thin active layers.[3][4] With a thin active layer, the average electron escape time is presumably much shorter than any spin reldtation times assocjated with the material and the effect of spin depolarization within the semiconductor is thus minimized. Secondly, the cathode may be used either in a $\mathrm{CW}$ mode or pulsed, with pulse lengths as short as 40 psec, [5] by appropriate choice of pump laser. The spin polarization is promptly reversible by reversing the helicity of the pump laser. No external magnetic fields are necessary for spin orientation. Finally, the source is necessarily extremely clean and well suited to the high vacuum environment associated with beam transport and acceleration.

Other methods for polarized electron production include:

- Electron scattering from unpolarized targets

- Photo-jonization of polarized atomic beans

- Fano effect (photo-iomization of unpolarized atons with circularly polarized radiation) 
- Optical pumping of a He discharge

- Field emission from W-EuS

Some of these sotrces are capable of achieving up to $90 \%$ polarization and their performance may be well suited to the needs of certain experiments.[1] However, the short pulse, high peak current and pulse to pulse spin reversal capabilities of the GaAs photocathode source make it uniquely suited for the needs of a high-current, short duty cycle accelerator such as that at the Stanford Linear Accelerator Center (SLAC) in Stanford, California. [6]

Perhaps the greatest shortcoming of the zinc-blende photocathode is the obtainable polarization level. The $50 \%$ polarization limit of the zinc-blende photocathode is inherent to its crystaj symmetry and related electronic band structure. There exist, however, semiconductors of appropriate crystal symmetry which should exhibit a theoretical $100 \%$ electron spin polarization. [7] These are the ternary chalcopyrites, of which $\mathrm{CdSiAs}_{2}$ is an example. These semiconductors have a natural crystal lattice of tetragonal symmetry rather than the cubic symmetry of the zinc-blende crystal. The band structure of the direct band gap chalcopyrites is appropriate for the possibility of $100 \%$ electron spin polarization by selective optical pumping of a single valence band. Unfortunately, work towards the development of chalcopyrite based photocathodes has achieved only modest success.[8]

Recent work towards enbancing the spin polarization from III-V senjiconductors has included the development of structures which incorporate a well defined strain into the crystal lattice of the active layer. When a direct band gap semiconductor of the zinc-blende type (e.g. GaAs) is uniaxially strained along one of the 
primary crystalline axes, the crystal symmetry is reduced from cubic to tetragonal and the band structure is modified in a manner which allows for a theoretical 100\% electron spin polarization just as for the chalcopyrites.

One method of straining a crystal is to mechanically apply an external stress. Experiments of this type have been performed and enhanced spin polarization was indeed observed with circularly polarized luminescence from strained GaSb[9] and, later, strained GaAs.[10] However, the application of mechanical stress is technically not a practical approach for a real pbotocathode in an ultra bigh vacuum environment.

Another method of incorporating strain into a crystal is by growing a heteroepitaxial layer of the material on a substrate possessing a slightly different lattice constant. In this case, the layer will grow pseudomorphically, constrained to the lattice constant of the substrate, until a certain thickness where the coherent strain energy within the layer is relieved through the generation of misfit dislocations. Although the original theoretical models for strained layer relaxation[11][12] predicted that relaxation would occur at thicknesses much less tban secessary for a practical photocathode, experiments showed that, in fact, large residual strains were possible in layers of usable thickness.[13][14] The critical thicknesses for relaxation found in these experiments agree reasonably well with a later madel for strain relaxation based on energy ba]ance.[15]

The first enhanced photo-emitted electron polarization from a photocathode employing a stzained hetero-epitaxial layer was reported in 1991.[16] This structure consisted of a thin $(100 \mathrm{~nm})$ active layer of $\mathrm{In}_{.12} \mathrm{Ga}_{.88}$ As grown on GaAs. Subse- 
quently, an enbancement of photo-emitted electron spin polarization was reported for a structure consisting of a strained layer of GaAs grown on $\mathrm{GaAs}_{(1-x)} \mathrm{P}_{x} \cdot[17]$ In each case, the active layer is under biaxial compression so that the normally degenerate 'heavy' hole and 'light' hole valence bands are split with the 'heavy' hole valence band lyjng closer to the conduction band. The latter struçture has a decided advantage over the former because the GaAs active layer has a larger band gap than the $\ln _{.12} \mathrm{Ga}_{.28} \mathrm{As}$, thus malking the attainment of a NEA surface easier. The success of the strained layer photocathodes warrants a research program to investigate the physical properties and operational behavior of the structures, with the possibility of improving photocathode performance.

One method of studying GaAs photocathode structures is through the detection of recombination radiation from optically pumped electrons (photoluminescence). The recombination radiation spectrom is related to the energy dependence and separation of the participating bands. Furthermore, if the polarization states of pump beam and recombination radiation are considered, information regarding the form of valence and conduction band wave functions may be obtained from the luminescence spectra. For example, the degree of circular polarization of the luminescence is related to the electron spin polarization of the photonexcited conduction electrons in the following manner.

$$
P_{\gamma}=P_{s}-P_{h} R \cos \omega
$$

Hexe, $P_{e^{-}}$is the electron spin polarization at the moment of photo-excitation to the conduction band and $P_{k}$ is the recombination radiation coupling factor. These two terms axe determined by the dipole transition selection rules for the appropriate 
band to band transitions and are identical at equivalent pump and recombination photon energy and direction. The term $R$ is a factor which account for any spin relaxation process and is assumed to be independent of photon energy. The angle $\omega$ is measured between the pump and luminescence photon directions. The relationship given in eq. (1.2) allows one to use circularly polarized luminescence as an indirect measure of electron spin polarization.

In this work, we study the polarized photoluminescence from a series of photocathodes consisting of a strained layer of GaAs grown on a fattice-mismatched $\mathrm{GaAs}_{(1-x)} \mathrm{P}_{x}$ buffer which is itself grown on a GaAs substrate. A different type of strained GaAs structure is also studied. This sample consists of a strained layer of $\mathrm{GaAs}$ on a $\ln _{x} \mathrm{Ga}_{(1-x)} \mathrm{P}$ buffer which is grown on a $\mathrm{GaP}$ substrate. Lastly, for reference purposes, an epitaxial GaAs structure possessing no lattice strain is studied. The total of nine GaAs photocathode structures have residual strain levels ranging from zero to $\sim 0.8 \%$ and active layer thicknesses ranging from 100 to 500 nm.

The photocathodes are characterized by both luminescence and X-ray diffraction measurements. X-ray diffraction data provides measurement of the lattice structure for the strajned GaAs layer and the strain-inducing buffer layer, while the luminescence spectra may be used a relative measure of the band gaps for these layers. Furthermore, the luminescence and X-ray diffraction data obtained from the strained GaAs layers and the strajn-inducing buffer layers may be correlated. Such correlation is carried out with the purpose of developing luminescence-based characterization techniques, because the luminescence mea- 
surements are obtained much more readily than the X-ray data. Examples of the use of the characterization techniques for prediction of photocathode electron spin polarization performance and photocathode wafer unjformity mapping are given.

Circularly polarized luminescence measurements acquired for the various photocathodes are used to infer the electron spin polarization. By investigating the dependence of electron spin polarization on strain and active layer thickness, it may be possible to elucidate the mechanisms responsible for the discrepancy between observed spis polarization values and the theoretical $100 \%$ limit. The luminescence measurements are complemented by spin polarization measurements made directly by photo-emission. Comparison of these two types of data may provide a means of distinguishing between effects associated with optical pumpingtof the actjve layer and those specific to the process of electron escape from the material.

In Chapter 2 of this work, the band structure of GaAs is described within the framework of a model based on a single electron traveling in a spatially infinite periodic lattice potential. Wave functions representative of the valence bands near $\Gamma$ are presented for the case of a non-strained lattice. The effects of Jattice strain, specifying to a state of biaxial compression, are then iacluded in the calculation of wave functions and important differences from the non-strained case are discussed.

Optical transition probabilitjes for both non-strained and biaxially compressed GaAs are presented in Chapter 3. The transition probabilities may be used to calculate the expected electron spin polarization and luminescence polarization for the case of circularly polarized optical pumping. The effects of non-zero electron momentum on the spin polarization for strained GaAs are considered at the end 
of this chapter.

In Chapter 4, the stracture of the various samples and special sample preparation techniques are described. The details of the optical pumping systems, luminescence detection hardware, optics and measurement techniques are presented. The X-ray diffraction apparatus and the techniques for measuring lattice strain are also described.

Chapter 5 presents the luminescence and X-ray diffraction data. Luminescence measurements nsed in conjunction with X-ray diffraction data are used to develop luminescence-based characterization techniques for sample structure. The room temperature luminescence polarization data is compared with spin polarization data from photo-emission experiments. Data taken at $\approx 78 \mathrm{~K}$ and $\approx 12 \mathrm{~K}$ atre also presented and discussed.

In Chapter 6, analysis of the data is presented. This analysis includes calculation of the deformation potentials $a, b$, and $d$ for GaAs. The observed agreenent with published values supports the assumptions of the model used in the analysis. Values of the spin relaxation factor $R$ are also determined for the samples. From sample to sample comparison of $R$, it is determined that spin relaxation accurs at the surface or in the surface band bending region of the samples. Conclusions drawn from the data analysis are summarized and possible implications with regard to future photocathode design are discussed. 


\section{Chapter 2}

\section{Band Structure of GaAs}

\subsection{Overview}

In this chapter we discuss the band structure of the zinc-blende crystal in the vicinity of the gamma point $(\Gamma)$. The electron wave functions are determined with the underlying assumption of a single electron moving in a spatially infinite periodic lattice. The epin orbit interaction is included in the Hamiltonian to account for the spin orbit coupling which is critical to the process of spin orientation by optical pumping.

After the electron wave functions near $\Gamma$ have been determined, strain is introduced into the system. A biaxial compression, consistent with that expected for lattice mismatched epitaxial growth on a (001) surface, takes the cubic lattice to a tetragonal symmetry. The Hamiltonian for the strained lattice is expressed in terms of the non-strained coordinates in the manner described by Pikus and Bjr.[18] The wave functions for the strained tattice are determined only after assuming that the strain dependent mixing terms dominate over $k$ dependent terms such that the latter may be ignored. The effect of nog-zero $k$ on the wave functions and optical orientation in the strained crystal is addressed at the end of Chapter 
3.

It will be shown that the incorporation of strain into the lattice has a significant effect on the valence bands near $\Gamma$. A knowledge of the wave functions for both the strained and non-strained cases is necessary to calculate the spin polarization expected from optical transitions under the condition of circularly polarized pump conditions. The theory relevant to the optical transitions and associated spin polarizations will be discussed in Chapter 3 .

\subsection{Band Structure of GaAs}

\subsubsection{Electrons in a Crystal Lattice}

The crystal structure of a semiconductor may be described by the onit cell for the lattice. Similarly, the Brillouin zone describes the reciprocal space of the unit cell. Figure 2.1 shows the Brillouin zome for the zinc-blende structure. This volume

encompasses the reduced wave vector $(\vec{k})$ space for the crystal. The symbols $\Gamma, X, L, \Sigma, \ldots$ represent special symmetry points and directions within the Brillouin zone. These symmetry points may be associated with band extrema or inflection points. The Gamma point ( $\Gamma$ ) corresponds to the condition $\bar{k}=0$ and, for GaAs, also to the band gap minimum.

The wave function for an electron in a crystal is properly expressed in the so called Bloch form[19] as shown in eq. (2.1).

$$
\Psi(\vec{r})=u_{n, \vec{k}}(\ddot{r}) e^{i \vec{k} \cdot \vec{r}}
$$

Here, $\vec{k}$ is related to the 'full' wave vector by $\vec{k}=\vec{k}_{f w l i}-2 \pi \sum m_{j} \hat{a}_{j}^{*}$, where $\hat{a}_{j}^{*}$ 


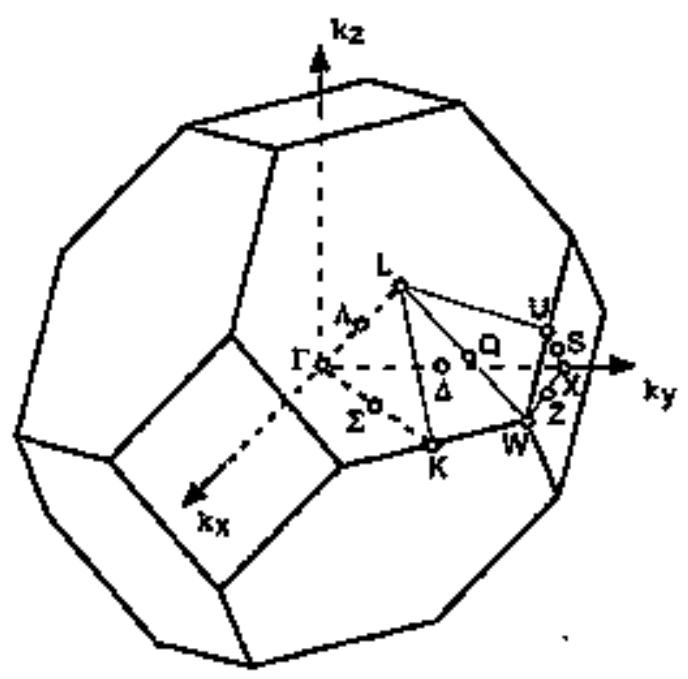

Figure 2.1: First Brillouin zone for the face-centered-cubic lattice. Special symmetry points and directions are labeled.

are the basis vectors for the reciprocal lattice and $m_{j}$ are appropriate integers such that $\left|\ddot{k}_{j}\right| \leq a_{j}^{*}$ for all $j$. The term $n$ in eg. (2.1) is a band index which is analogons to the atomic-orbital shell index. This wave function describes a plane wave modulated by the function $u_{n, k}(\vec{r})$ which carries the periodicity of the crystal lattice and, in general, depends on $\vec{k}$.

Since the electron wave fonctions are so intimately related to the symmetry of the crystal lattice, much can be learned about the electronic properties of the crystal within the framework of group theory without explicit calculation of the wave functions. In fact, using this approach, D'yakonov and Perel' showed that the maximum possible level of optical orientation of electron spin in GaAs is indeed limited to $50 \%$.[20] The 50\% result may also be obtajned by solving the eigenvalue equation for the valence electron wave functions directly and then calculating the appropriate optical transition probabilities. The procedure used to solve the eigen- 
value equation was introduced by Kane to describe $\mathrm{InSb}[21]$ and is known as the $k \cdot p$ method.

\subsubsection{The $k \cdot p$ Method}

The $k \cdot p$ method allows one to find analytic solutions for the valence wave functions near $\Gamma$ within a reduced manifold. The single electron Schroedinger equation is given by

$$
H \Psi_{n}=\left(\frac{p^{2}}{2 m}+V(\bar{r})\right) \Psi_{n}=E_{m} \Psi_{n}
$$

The spin orbit interaction is onitted here but will be included at a later point. The wave function $\Psi_{n}$ must be of the form shown in eq. (2.1). Inserting the Bloch function into eq. (2.2) and allowing the momentum operator to act on the $e^{i \sqrt{2}, r^{*}}$ portion of the term, one obtains

$$
\left(\frac{p^{2}}{2 m}+\frac{\hbar}{m} \tilde{k} \cdot \vec{p}+\frac{\hbar^{2} k^{2}}{2 m}+V(\vec{r})\right) u_{n, \vec{k}}=E_{\mathrm{n}} u_{n, \vec{k}} .
$$

The functions $u_{n} \vec{k}_{0}$ form a complete set for any fixed $\vec{k}=\vec{k}_{o}$ and therefore constitute a basis for all $u_{n, k}$. Because eigenstates in the vicinity of $\Gamma(\vec{k}=0)$ are of interest, a basis is formed with set of functions $u_{n, 0}$. These functions are solutions to eq. (2.3) for $\vec{k}=0$ and they are known from the set of irreducible representations for the symmetry point $\Gamma$.

Next, the general functions $u_{n, k}$ are expressed in terms of the basis functions as

$$
u_{n, \vec{k}}=\sum_{n^{\prime}} c_{n, n^{\prime}}(\vec{b}) u_{n^{\prime}, 0}
$$

where the coefficients $c_{n, n^{\prime}}$, in general, depend on the wave vector $\vec{k}$. The eigenvalue equation given in eq. (2.3) is rewritten with $u_{n, E}$ expressed in terms of the basis 
functions on the left hand side, and a matrix equation then constructed in the following manner.

$$
\sum_{n^{\prime}}\left\langle u_{n^{\prime}, 0}\left|\left(\frac{p^{2}}{2 m}+\frac{\hbar}{m} \vec{k} \cdot \vec{p}+\frac{\hbar^{2} \vec{k}^{2}}{2 m}+V(\vec{r})\right\}\right| u_{s^{\prime}, 0}\right) c_{n, n^{\prime}}(\vec{k})=E_{n_{n}, \mathcal{k}^{\prime} c^{\prime \prime}, n}
$$

The matrix equation given in eq. (2.5) can, in principle, be diagonalized to find the various eigenstates and eigenenergies. However, as expressed, it spans a basis consisting of all functions which transform under the symmetry group of $\Gamma$. The next step in the $k \cdot p$ method is therefore to reduce the matrix to a manifold containing only the basis functions of interest. The technique used is known as the Lowdin perturbation method.[22] Following this method, the basis is separated into two subsets. The states within the first subset ase assumed to interact strongly with one another but only weakly with the states of the latter. The interaction between the two subsets is removed by standard perturbative methods taken to second order. At this point, the second subset is ignored and the working manifold is represented by the first subset. This manifold may then be diagonalized.

\section{$2.2 .3 k \cdot p$ Matrix for GaAs}

The tetrahedral bonding structure for GaAs (and all zinc-blende crystals) is composed of atomic $S$ and $P$ orbitals. The valence states near the band gap have this symmetry and the basis states for the $k \cdot p$ mabifold are chosen to be $S X Y Z$. The 
$k \cdot p$ mattix within this basis is given by

$$
\mathbf{H}_{k^{\prime} \cdot \mathrm{p}}=\left(\begin{array}{cccc}
S & X & Y & Z \\
E_{c}+A k^{2} & B k_{y} k_{x} & B k_{x} k_{x} & B k_{y} k_{z} \\
+\hbar^{2} k^{2} / 2 m & +i P k_{x} & +i P k_{y} & +i P k_{x} \\
& & & \cdot \\
B k_{y} k_{z} & E_{y}+M\left(k_{y}^{2}+k_{z}^{2}\right) & N k_{z} k_{y} & N k_{z} k_{z} \\
-i P k_{x} & +L k_{x}^{2}+\hbar^{2} k^{2} / 2 m & & \\
& & & \\
B k_{x} k_{x} & N k_{x} k_{y} & E_{u}+M\left(k_{x}^{2}+k_{z}^{2}\right) & N k_{y} k_{x} \\
-i P k_{y} & & +L k_{y}^{2}+\hbar^{2} k^{2} / 2 m & \\
& & & \\
-i P k_{x} k_{y} & N k_{x} k_{z} & N k_{y} k_{z} & E_{v}+M\left(k_{x}^{2}+k_{y}^{2}\right) \\
& & & +L k_{z}^{2}+\hbar^{2} k^{2} / 2 m
\end{array}\right)
$$

The terms $E_{c}$ and $E_{v}$ are the gamma point energies of the conduction band and uppermost valence bands, respectively. The constants $A, B, L, M, N$ come from the step of perturbative removal of the weakly interacting basis states and the explicit manner in which they are calculated within the Löwdin procedure is given by Kane. These constants are important in the effective mass description of the band structure, and their values may be determined empirically from cyclotron resonance experiments.[23] The constant $P$ is the result of the $k \cdot p$ interaction within the $S X Y Z$ basis and is defined as

$$
P=-i \frac{\hbar}{\pi}\left\langle S\left|p_{2}\right| Z\right\rangle
$$


The term $P$ has been found to have a fairly large value of approximately $P=(20$ $\left.\mathrm{eV} \times \hbar^{2} / 2 m\right)^{1 / 2}$ for all of the various III-V compounds.[24]

\subsubsection{Spin Orbit Interaction}

The spin orbit interaction strongly influences the valence wave functions for GaAs. It is this interaction which couples the electron spin to the symmetry carrying component, $u_{n, k}$, of the wave function thus providing the possibility for optical orientation of spin. The spin orbit interaction is given by

$$
H_{s a}=\frac{\hbar}{4 m^{2} \mathrm{c}^{2}}(\nabla V(\vec{r}) \times \vec{p}) \cdot \sigma,
$$

where $\sigma$ represents the Pauli matrices for a spin $1 / 2$ particle. When spin is considered, the $S X Y Z$ basis is naturally expanded to the eightfold basis

$$
S \uparrow, S \downarrow, X \uparrow, X \downarrow, Y \uparrow, Y \downarrow, Z \uparrow, Z \downarrow
$$

The expansion of the basis to include spin does not effect the $k \cdot p$ matrix shown in eq. (2.6) except to produce the sbown matrix for both spin up and spin down states with no interaction between opposite spin states.

The spin orbit interaction must be included in the Schroedinges equation before the traveling wave component of $\Psi(\vec{r})$ has been removed. When this is done, the interaction produces a second term due to the action of the momentum operator on the traveling wave component of the Bloch function. The two terms which will act on the functions $u_{n, k}$ are

$$
H_{* 0}^{1}=\frac{\hbar}{4 m^{2} c^{2}}(\nabla V(\vec{r}) \times \vec{p}) \cdot \sigma
$$


and

$$
H_{s o}^{2}=\frac{\hbar}{4 m^{2} c^{2}}(\nabla V(\vec{r}) \times \vec{k}) \cdot \sigma .
$$

The $\vec{k}$ dependent term $H_{s o}^{2}$ is neglected here because, near band edge, this term will be much smaller than $H_{s a}^{1}$. Another approximation in obtaining an interaction matrix which can be diagonalized is to neglect the terms which are generated in the Löwdin perturbation procedure by the spin orbit interaction $H_{s o^{*}}^{l}$. These neglected terms, along with some second order perturbation terms, are linear in $\vec{k}$ and cause a shift of the valence band maxima from the $\Gamma$ point when appropriately accounted for.[21]

The spin orbit interaction terms included in the interaction matrix are of the form

$$
\begin{gathered}
\left\langle X \downarrow(\uparrow)\left|H_{s o}^{1}\right| Z \uparrow(\downarrow)\right\rangle=+(-) \Delta, \\
\left\langle Y \downarrow(\dagger)\left|H_{s 0}^{1}\right| Z \uparrow(\downarrow)\right\rangle=+(-) i \Delta, \\
\left\langle X \uparrow(\downarrow)\left|H_{s o}^{1}\right| Y \uparrow(\downarrow)\right\rangle=\Delta .
\end{gathered}
$$

This interaction requires the eigenfunctions to be states of $J$ and $m_{j}$. Kane obtained solutions for the complete interaction, $H_{k \cdot p}+H_{s a}^{1}$, by rotating the coordinate system so that $\hat{z}$ lies along $\vec{k}$. The rotation transformation matrices are given by

$$
\left(\begin{array}{l}
\dagger^{\prime} \\
\downarrow^{\prime}
\end{array}\right)=\left(\begin{array}{cc}
e^{-i \phi / 2}\left(\cos \frac{\theta}{2}\right) & e^{i \phi / 2}\left(\sin \frac{\theta}{2}\right) \\
-e^{-i \phi / 2}\left(\sin \frac{\theta}{2}\right) & e^{i \phi / 2}\left(\cos \frac{\theta}{2}\right)
\end{array}\right)\left(\begin{array}{l}
\dagger \\
\downarrow
\end{array}\right)
$$

and

$$
\left(\begin{array}{l}
S^{\prime} \\
X^{\prime} \\
Y^{\prime} \\
Z^{\prime}
\end{array}\right)=\left(\begin{array}{cccc}
1 & 0 & 0 & 0 \\
0 & \cos \theta \cos \phi & \cos \theta \sin \phi & -\sin \theta \\
0 & -\sin \phi & \cos \phi & 0 \\
0 & \sin \theta \cos \phi & \sin \theta \sin \phi & \cos \theta
\end{array}\right)\left(\begin{array}{l}
S \\
X \\
Y \\
Z
\end{array}\right),
$$


where $\theta$ is the angle of the $z^{\prime}$ axis from the fixed $z$ axis and $\phi$ is the azimuthal angle of the projection of $z^{\prime}$ on the fixed $x-y$ plane. The fixed (unprimed) coordinate Bystem is defined by and coincident with the crystal axes.

The rotation transformations do not affect the isotropic spin orbit interaction matrix, but the $k \cdot p$ interaction matrix is transformed so that all terms are effectively rewritten with $k_{z}=k$ and $k_{x}=k_{y}=0$. The interaction matrix for the rotated coordinate system may be diagonalized by transforming to the following basis.

$$
\begin{aligned}
i S^{\prime} \uparrow^{\prime}, & i S^{\prime} \downarrow^{\prime} \\
\sqrt{\frac{1}{2}}\left(X^{\prime}+i Y^{\prime \prime}\right) \uparrow^{\prime}, & -\sqrt{\frac{1}{2}}\left(X^{\prime}-i Y^{\prime}\right) \downarrow^{\prime} \\
\sqrt{\frac{1}{6}}\left(X^{\prime}+i Y^{\prime}\right) \downarrow^{\prime}-\sqrt{\frac{2}{3}} Z^{\prime} \uparrow^{\prime}, & -\sqrt{\frac{1}{6}}\left(X^{\prime}-i Y^{\prime}\right) \uparrow^{\prime}-\sqrt{\frac{2}{3}} Z^{\prime} \downarrow^{\prime} \\
\sqrt{\frac{1}{3}}\left(X^{\prime}+i Y^{\prime \prime}\right) \downarrow^{\prime}+\sqrt{\frac{1}{3}} Z^{\prime} \uparrow^{\prime}, & \sqrt{\frac{1}{3}}\left(X^{\prime}-i Y^{\prime}\right) \uparrow^{\prime}-\sqrt{\frac{1}{3}} Z^{\prime} \downarrow^{\prime}
\end{aligned}
$$

The full wave functions have a traveling wave term appended to the above functions to form the Bloch wave functions. The interaction matrix is diagonal in this basis except for a coupling between the $m_{j}= \pm 1 / 2$ conduction states and the $m_{j}= \pm 1 / 2$ valence states. This coupling arises from the term

$$
\left\langle S^{\prime} \uparrow^{\prime}\left(\downarrow^{\prime}\right)\left|H_{k-p}+H_{s o}\right| Z^{\prime} \uparrow^{\prime}\left(\downarrow^{\prime}\right)\right\rangle=P k
$$

where $P$ is defined in eq. (2.7).

Table 2.2.4 summarizes the wave functions and respective energies obtained with the $k \cdot p$ method for electrons in the vicinity of $\Gamma$. The energies are seen to be quadratic in $k$, and each band has an effective mass associated with it. The $\left\langle\frac{3}{2}, \frac{3}{2}\right\rangle$ and $\left|\frac{3}{2}, \frac{1}{2}\right\rangle$ valerice bands are referred to as the 'beavy hole' and 'light hole' bands respectively due to their effective masses $m_{h k}$ and $m_{i k}$. The $\left(\frac{1}{2}, \frac{1}{2}\right)$ 


\begin{tabular}{|c|c|c|}
\hline$S_{1 / 2}$ & $\left\{\frac{1}{2}, \frac{+1}{2}\right\rangle$ & $\left(i S^{\prime} \uparrow^{\prime}\right) e^{i k x^{\prime}}$ \\
\hline & $\left|\frac{1}{2}, \frac{-1}{2}\right\rangle$ & $\left(i S^{\prime} \downarrow^{\prime}\right) e^{i k z^{\prime}}$ \\
\hline
\end{tabular}

$$
\begin{array}{cccc}
P_{3 / 2} & \left|\frac{3}{2}, \frac{+3}{2}\right\rangle & \left(\sqrt{\frac{1}{2}}\left(X^{\prime}+i Y^{\prime}\right) \uparrow\right) e^{i k z^{\prime}} & E_{v}+\hbar^{2} k^{2} / 2 m_{h h} \\
& \left|\frac{3}{2}, \frac{-3}{2}\right\rangle & \left(-\sqrt{\frac{1}{2}}\left(X^{\prime}-i Y^{\prime}\right) \downarrow^{\prime}\right) e^{i k z^{\prime}} & \\
& \left|\frac{3}{2}, \frac{+1}{2}\right\rangle & \left(\sqrt{\frac{1}{6}}\left(X^{\prime}+i Y^{\prime}\right) \downarrow^{\prime}-\sqrt{\frac{2}{3}} Z^{\prime} \dagger^{\prime}\right) e^{i k z^{\prime}} & E_{v}+\hbar^{2} k^{2} / 2 m_{h h} \\
& \left|\frac{3}{2}, \frac{-1}{2}\right\rangle & \left(-\sqrt{\frac{1}{6}}\left(X^{\prime}-i Y^{\prime}\right) \uparrow^{\prime}-\sqrt{\frac{2}{3}} Z^{\prime} \downarrow^{\prime}\right) e^{i k z^{\prime}} & \\
. & & & \\
P_{1 / 2} & \left|\frac{1}{2}, \frac{+1}{3}\right\rangle & \left(\sqrt{\frac{1}{3}}\left(X^{\prime}+i Y^{\prime}\right) \downarrow^{\prime}+\sqrt{\frac{1}{3}} Z^{\prime} \uparrow^{\prime}\right) e^{i k z^{\prime}} & E_{v}-\Delta+\hbar^{2} k^{2} / 2 m_{8 \alpha} \\
& \left|\frac{1}{2}, \frac{-1}{2}\right\rangle & \left(\sqrt{\frac{1}{3}}\left(X^{\prime}-i Y^{\prime}\right) \uparrow^{\prime}-\sqrt{\frac{1}{3}} Z^{\prime} \downarrow^{\prime}\right) e^{i k z^{\prime}} & \\
\hline
\end{array}
$$

Table 2.1: Conduction band and valence band electron wave functions and energies in the vicinity of $\Gamma$ as calculated within the $k \cdot p$ scheme. 


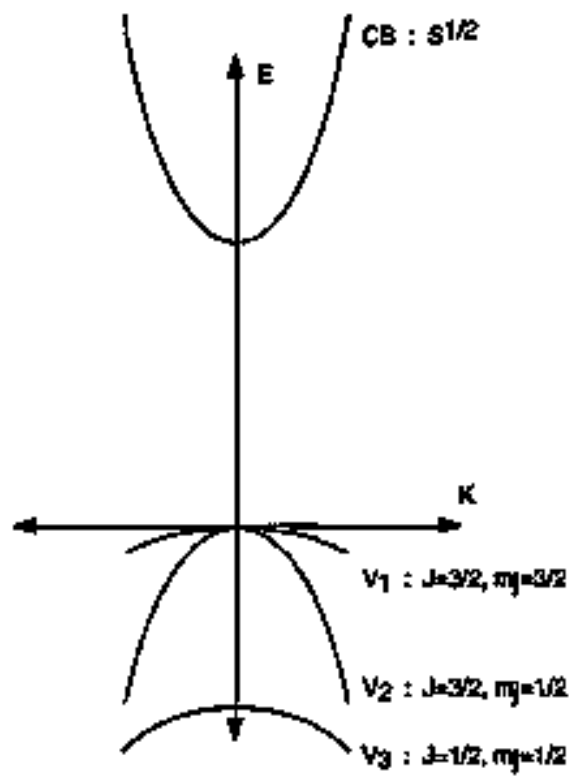

Figure 2.2: Band structure near $\Gamma$ of non-strained GaAs.

valence band is referred to as the 'split off' or 'spin orbit' band because of the energy splitting $\Delta$ which arises from the spin orbit interaction. The conduction and valence band effective masses bave been experimentally measured and are approximately $m_{c}=.067 m, m_{\hbar h}=-.50 m, m_{l h}=-.068 m$ and $m_{s a}=-.13 m,[25]$ where $m$ is the electron mass. Figure 2.2 shows a diagram of the band structure in the vicinity of $\Gamma$.

\subsection{Strain Induced Effects near $\Gamma$}

\subsubsection{Strain Interaction Matrix}

When a crystal is deformed, its symmetry is chazged as is the set of transformation operations which describe the point group of the lattice. The change in symmetry 
will have important effects on the wave functions which describe electrons near the $\Gamma$ point. Because the band structure should remain unchanged in the limit of vanishingly small strains, one is motivated to express the effects of the strain as a perturbation to the existing Hamilitonian. The effects of a lattice strain are thus incorporated by expressing the operators of the strained lattice Haniltonian in terms of the non-distorted lattice operators. [18] This procedure leads to to the generation of the following terms.

$$
\begin{gathered}
D=\sum_{i, j} D_{i j} \epsilon_{i j} \\
D_{i j}=\frac{\hbar^{2}}{m} \frac{\partial^{2}}{\partial x_{i} \partial_{x_{j}}}+V_{i j}-\frac{\hbar}{m} P_{i} k_{j} \\
V_{i j}(\vec{r})=\left.\frac{\partial}{\partial \epsilon_{i j}} V((1+\hat{\epsilon}) \cdot \vec{r})\right|_{i \rightarrow 0}
\end{gathered}
$$

Here, the $\epsilon_{i j}{ }^{\prime} s$ are elements of the strain tensor $\hat{\epsilon}$. The new Hamiltonian terms $\left(D_{i j}\right)$ are carried out through the $k \cdot p$ interaction generated during the removal of the traveling wave component of the Bloch function, but they do not include any terms generated during the Löwdin procedure. Those interactions are all higher order in strain and therefore considered negligible.

The strain Hamiltonian terms $\left(D_{i j}\right)$, when considered within the $S X Y Z$ basis, yjeld the spin degenerate interaction matrix

$$
\mathrm{D}=\left(\begin{array}{cccc}
S & X & Y & Z \\
0 & 0 & 0 & 0 \\
0 & -a \epsilon+b\left(3 \epsilon_{x x}-\epsilon\right) & d \epsilon_{x y} & d \epsilon_{x y} \\
0 & d \epsilon_{x y} & -a \epsilon+b\left(3 \epsilon_{y y}-\epsilon\right) & d \epsilon_{y z} \\
0 & d \epsilon_{x z} & d \epsilon_{y z} & -a \epsilon+b\left(3 \epsilon_{z z}-\epsilon\right)
\end{array}\right) \text {, }
$$


where $\epsilon=$ Trê, and the terms $a, b$, and $d$ are deformation potentials which relate lattice strain with hydrostatic and band-splitting energy shifts.

\subsubsection{Biaxial Compression}

In order to evaluate the effect of the strain matrix (D) on the band struicture, the form of the strain tensor must be specified and applied to eq. (2.22). For the case of a layer of (001) GaAs grown pseudomorphically on a $\mathrm{GaAs}_{(1-x)} \mathrm{P}_{x}$ (or $\mathrm{In}_{\mathrm{r}} \mathrm{Ga}_{(1-x)} \mathrm{P}$, $x<.49)$ substrate, the lattice strain is ideally a pure biaxial compression within the plane of growth. This state of lattice strain is characterized by a strain tensor with the following components.

$$
\begin{gathered}
\epsilon_{x \pi}=\epsilon_{y y} \\
\epsilon_{z x}=\frac{-2 c_{12}}{c_{11}} \epsilon_{x \tau} \\
\epsilon_{x y}=\epsilon_{y z}=\epsilon_{x z}=0
\end{gathered}
$$

The relationskip given in eq. (2.24) between the strain along the direction of crystal growth $\left(\epsilon_{x x}\right)$ and that in the growth plane $\left(\epsilon_{x x}, \epsilon_{y y}\right)$ is found from the stress-strain relationship by assuming there is no applied stress in the $z$ direction. The terms $c_{11}$ and $c_{12}$ are elastic stiffness constants which relate stresses and strains. It is worth noting that the bjaxial compression described bere is equivalent to a uniaxial dilation a]ong the [001] direction.

\subsubsection{Solutions at $\mathrm{r}$}

The strained lattice interaction matrix given by eq. (2.22) becomes greatly simplified under the condition of a pure biaxial compression. Ideally, one would like to 
obtain a set of solutions for the entire Hamiltonian $H=H_{k \cdot p}+H_{s o}+D$. Hotwever a tractable solntion of the entire interaction matrix for arbitrary values of $k$ is not possible. Therefore, solutions are obtajned for the case $k=0$ and the effects of small non-zero electron momentum are subsequently considered. For $k=0$ the interaction matrix is neariy diagonal upon transformation to the $\left[J, m_{j}\right\rangle$ basis with the $\hat{z}$ direction defined along the uniaxial strain.

$$
\mathbf{H}_{k, p}(k=0)+\mathbf{H}_{\alpha c}+\mathbf{D}=\left(\begin{array}{cccc}
S_{1 / 2,1 / 2} & P_{3 / 2,3 / 2} & P_{3 / 2,1 / 2} & P_{1 / 2,1 / 2} \\
E_{g} & 0 & 0 & 0 \\
0 & -\delta_{h}-\frac{1}{2} \delta_{s} & 0 & 0 \\
0 & 0 & -\delta_{h}+\frac{1}{2} \delta_{s} & \frac{1}{\sqrt{2}} \delta_{s} \\
0 & 0 & \frac{1}{\sqrt{2}} \delta_{s} & -\Delta-\delta_{h}
\end{array}\right)
$$

The subscripts on the atomic $S$ and $P$ symbols identify $J$ and $m_{j}$, respectively. The term $\delta_{h}$ represents the shift of valence bands relative to the conduction band due to the hydrostatic component of the lattice strain, and $\delta$, represents the splitting of the uppermost valence bands due to the shear component (uniaxial nature) of the strain. Expressed in terms of the deformation potentials and the in-plane compressive strain, these energy shifts are

$$
\delta_{h}=2 a \frac{c_{11}-c_{13}}{c_{11}} \varepsilon_{x x}
$$

and

$$
\delta_{s}=-2 b \frac{c_{11}+2 c_{12}}{c_{11}} \epsilon_{x x} .
$$

The strain $\epsilon_{x x}$ is that in the plane of growth along the [100] and [010] directions.

The interaction matrix shown in eq. (2.26) is not completely diagonal. It is reasonable to neglect the mixing between the $P_{3 / 2, \pm 1 / 2}$ and $P_{1 / 2, \pm 1 / 2}$ states for 
$\delta_{\Delta} \ll \Delta$. This condition is met reasonably well for the range of lattice strains encountered in this study. Furthermore, the mixing will have no effect upon the electron polarization expected from optical transitions from each specific band to the conduction band when pumping with circularly polarized light. Aithough the mixing will be ignored with regard to exact wave function solutions at $k=0$, the effect of such mixing on the energies of the valence states will be considered.

The energies for the valence states at $k=0$ ase given by $E_{3 / 2,3 / 2}=-d_{h}-\delta_{s} / 2$, $E_{3 / 2,1 / 2}=-\delta_{h}+\delta_{s} / 2+\delta_{s}^{2} / 2 \Delta_{3}$ and $E_{1 / 2,1 / 2}=-\Delta-\delta_{h}-\delta_{s}^{2} / 2 \Delta$ as expanded to second order in $\delta_{s}$. The band structure near $\Gamma$ for a biaxial compressive strain is shown in Figure 2.3. Both the band splitting energy $\delta$, and the hydrostatic energy shift $\delta_{h}$ are seen. Under a biaxial compression, the effective masses of the two upper valence bands near $\vec{k}=0$ become highly anisotropic, so that the constant energy surfaces are expressible as ellipsoids symmetric about the effective uniaxial strain axis $(\hat{z})$. The $m_{j}=3 / 2$ band has an elljptical constant energy surface with major axis along the strain axis, while the major axis for the $m_{j}=1 / 2$ band is orthogonal to the strain axis. The direction-averaged effective mass for each of the valence bands is nearly identical though, as depicted in Figure 2.3, and is approximately $m_{\frac{2}{2}, \frac{3}{2}} \approx m_{\frac{2}{2}, \frac{1}{2}} \approx .16 \mathrm{~m}$.

The effect of a biaxial compression on the valence band wave fumctions at $\Gamma$ is actually two fold. First, the uppermost lying valence bands which comprise the $p_{3 / 2}$ multiplet are split at $\Gamma$ allowing selective optical pumping at the band gap minimum. Secondly, the lattice strain acts to provide a fixed quantization axis for the orbital angular momenturn, and thus for the electron spin (through the spin 


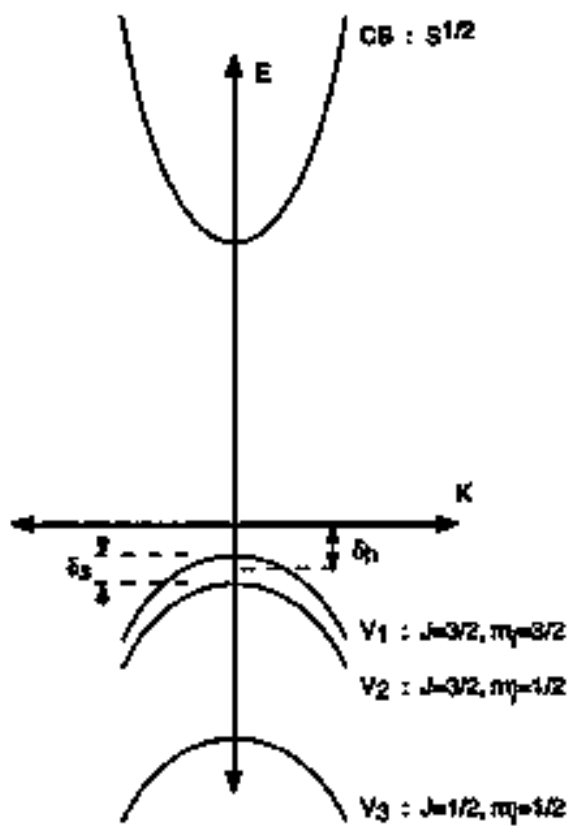

Figure 2.3: Band structure near $\Gamma$ of GaAs under bjaxial compression. orbit interaction), along the direction of the effective uniaxial strain. 


\section{Chapter 3}

\section{Optical Transitions and Spin Polarization -}

\subsection{Overview of Transition Probabilities}

A valence level electron in GaAs near the band gap minimum may be optically excited to the condaction band through the absorption of a single photon. This transition requires that the excitation photon be of sufficient energy to excite the electron across the band gap. Because the valence band maximum and conduction band minimum correspond to the same symmetry point in phase space (F), electrons may experience optical transition across the band gap minimum without the participation of a momentum carrying phonon. These transitions are known as vertical transitions. The respective atomic $S$ and $P$ symmetries of the conduction and valence states are appropriate for an optical electric dipole transition.

A full expression for the probability of transitions from valence level to conduction level involves many factors, and is given as[26]

$$
\frac{d P}{d E}=A M_{J_{i}, \rho_{c}}^{2}(E+h \nu) \rho_{v}(E)\left(F_{v}-F_{c}\right) \delta\left(\vec{k}_{c}-\vec{k}_{v}\right) .
$$

The terms $\rho_{c}(E+h \nu)$ and $\rho_{v}(E)$ refer to the density of states of the conduction and valence bands respectively. $F_{c}$ is the Fermi-Dirac distribution function for a conduction electron state at energy $E+h \nu$, and $F_{v}$ represents the probability of 
an occupied electron valence state at energy $E$. The delta function, $\delta\left(\vec{k}_{c}-\vec{k}_{v}\right)$, is a statement of conservation of crystal momentum. The term $A$ is a constant for a specific photon energy. Finally, the term $M_{f, i}$ is the dipole interaction matrix element between injtial and final electron states.

The photon-emitting conduction to valence transition probability for the same photon energy will differ in the Fermi-Dirac functions for electrons and holes. The expression for the downward transition is[26]

$$
\frac{d P}{d E}=A^{\prime} M_{f, i}^{2} \rho_{c}(E+h \nu) \rho_{\nu}(E) F_{c}\left(1-F_{v}\right) \delta\left(\vec{k}_{c}-\vec{k}_{v}\right)
$$

It is easy to show that the ratio of photon absorption to photon emission probability is a valence band independent term given by

$$
\frac{(d P / d E)_{\text {abtorb }}}{(d P / d E)_{\text {emit }}}=\frac{A}{A^{t}} \frac{F_{y}-F_{c}}{F_{c}\left(1-F_{y}\right)}=\frac{A}{A^{s}}\left(e^{k+/ k_{B} T}-1\right)
$$

The relationship between photon absorbing and photon emitting transitions given in eq. (3.3) is equivalent to a statement of detailed-balance given by Van Roosbroeck and Shockley.[27] It should be true for any specific photon energy, direction, and polarization state as long as there exists thermal equilibrium or quasiequilibrium in each of the bands.

\subsection{Spin Polarization from Optical Pumping}

\subsubsection{Formalism}

The spin state of an ensemble of electrons is quantified in terms of spin polarization. Electron spin is described by a two-state system which is referenced to a particular 
quantization axis. The spin polarization is a measure of the relative difference in population between the two independent spin states. If a quantization direction is defined, then the spin polarization atong that direction may be expressed as

$$
P_{e-}=\frac{N_{\uparrow}-N_{\downarrow}}{N_{\uparrow}+N_{\downarrow}}
$$

where $N_{\uparrow}$ and $N_{7}$ correspond to the number of electrons in spin states parallel and anti-parallel to the quantization direction.

A formal method of characterizing spin polarization is provided by the creation of a spin density matrix.[1] Given a population of ejectrons which may occupy a set of spin states $\Psi_{n}$ the density operator $f$ is defined as

$$
f=\sum_{\mathbf{n}} N_{n}\left|\Psi_{n}\right\rangle\left\langle\Psi_{n}\right|
$$

where $N_{n}$ is the number of electrons in state $\Psi_{n}$. The interaction of the density operator within a spin basis will form a spin density matrix. A quantization axis must be defined for the states $m_{+1 / 2}, m_{-1 / 2}$ which form the spin $1 / 2$ basis. The spin density matrix may then be formed as

$$
f_{m, m^{\prime}}=\sum_{n} N_{n}\left\langle m \mid \Psi_{n}\right\rangle\left\langle\Psi_{n} \mid m^{n}\right\rangle
$$

The average spin polarization is extracted from this matrix with the Pauli-spin matrices. [1]

$$
\vec{P}_{e^{-}}=\frac{\operatorname{Tr}(f \cdot \vec{\sigma})}{\operatorname{Tr}(f)}
$$

Here, $\vec{\sigma}=\sigma_{x} \hat{x}+\sigma_{y} \hat{y}+\sigma_{x} \hat{z}$ and $\sigma_{x}, \sigma_{y}, \sigma_{z}$ are the Pauli-spin matrices as standardly defined for a spin quantization axis lying along $\hat{z}$. 


\subsubsection{Optical Transitions to Conduction Band}

When discussing the spin polarization resulting from optical transitions from a valence state to a conduction state, the spin density matrix formalism may still be used. However, the appropriate density operator is given by

$$
\left.\left.f=\sum_{n} N_{s}|\mathbf{D}| \bar{\Psi}_{n}\right\rangle\left\langle\bar{\Psi}_{n}\right| \mathbf{D}\right] .
$$

The wave functions $\Psi_{n}$ correspond to a given valence band and $\mathbf{D}$ is the electric dipole operatox corresponding to the absorbed radiation.

The spin density matrix is formed within the basis defined by the conduction states of $S \uparrow$ and $S \downarrow$ but, in order for the matrix elements to be calculated, a specific dipole operator must be chosen. The matrix will be calculated for circularly polarized light with the quantization direction for the spin basis, $\hat{z}$, placed along the photon direction. Pure left circularly polarized $\left(\sigma^{+}\right)$radiation is chosen for the calculation. A separate spin density matrix will be calculated for transitions from each of the two uppermost valence bands, yjelding individual results for the expected spin polarization. Because the excitation photon fixes the quantization axis in space, the valence band wave functions must be expressed in the same caordinate system by using the rotation matrices given in eqs. (2.15) and (2.16). The spin density matrices are

$$
f^{(1)}=\frac{1}{12}\left(\begin{array}{cc}
\frac{3}{4} \sin ^{2} \theta & -\frac{3}{8} \sin 2 \theta e^{i \phi} \\
\frac{2}{8} \sin 2 \theta e^{i \phi} & \frac{3}{4}+\frac{9}{4} \cos ^{2} \theta
\end{array}\right)
$$


and

$$
f^{(2)}=\frac{1}{12}\left(\begin{array}{cc}
1-\frac{3}{4} \sin ^{3} \theta & \frac{3}{4} \sin 2 \theta e^{i \phi} \\
\frac{3}{4} \sin 2 \theta e^{i \phi} & \frac{9}{4} \sin ^{2} \theta
\end{array}\right),
$$

where the superscripts (1) and (2) refer to transitions from the $\left|\frac{3}{2}, \frac{3}{2}\right\rangle$ and $\left|\frac{3}{2}, \frac{1}{2}\right\rangle$ bands, respectively. The angle $\theta$ in eqs. (3.9) and (3.10) comes from the rotation matrices given in eqs. (2.15) and (2.16). It is the angle between the quantization axis of the valence wave functions and the axis defined by the absorbed photon beam.

The spin polarization of the conduction electrons excited from each valence band is caiculated from the spin density matrices using eq. (3.7) and is found to be

$$
P_{e-}^{(1)}=-\frac{2 \cos ^{2} \theta}{1+\cos ^{2} \theta}
$$

and

$$
P_{\mathrm{e}}^{(2)}=\frac{2-6 \sin ^{2} \theta}{2+3 \sin ^{2} \theta}
$$

where the orientation is $\vec{P}=P_{z} \hat{z}$ such that the spin polarization is along the excitation photon direction in both cases.

The spin polarizations found in eqs. (3.11) and (3.12) refer to transitions from a specific pair of valence states. That is, the polarization calculated for each valence band is valid for excitations from a valence state of specified wave vector $\vec{k}$. The polarization actually expected for a given photon energy $h \nu$ must be derived from a spin density matrix which includes integration over all allowed transitions. Integration is simplified by approximating the constant energy surfaces 
of the valence bands as spherically symmetric. In this way, a given photon energy corresponds to a specific magnitude of wave vector involved in the transition. The averaging of spin density matrix elements over all allowed transitions is then simply an average over wave vector direction.

$$
F_{m, m^{\prime}}=\int d \Omega_{k} f_{m, m} r(h)
$$

Here, $F_{m, m^{\prime}}$ is the spin density matrix element after averaging over the solid angle $\Omega_{k}$.

\section{Non-Strained GaAs}

The spin density matrix elements $f_{m, m}$ for the case of non-strained GaAs have a dependence on the polar angle $\theta$ between the spin quantization axis ( $\hat{z}$ ) defined by the absorbed photon direction and the quantization axis for the valence states $(\vec{k})$. This angle is integrated over when finding the terms $F_{m, m^{\prime}}$ from eq. (3.13). The result of the integration for the non-strained case gives

$$
F^{(1)}=\frac{1}{12}\left(\begin{array}{cc}
1 / 2 & 0 \\
0 & 3 / 2
\end{array}\right)
$$

and

$$
F^{(2)}=\frac{1}{12}\left(\begin{array}{cc}
1 / 2 & 0 \\
0 & 3 / 2
\end{array}\right)
$$

The spin polarization is then calculated from eqs. (3.14) and (3.15) in the manner of eq. (3.7) giving $P_{t^{+}}^{(1)}=P_{e^{-}}^{(2)}=-0.5$. 
When a non-strained sample of GaAs is optically pumped neat band edge, both of the uppermost valence bands contribute to the overall photo-excited conduction electron population. The resulting spin polarization will therefore be an average of the spin polarized population from each valence band to conduction band transition. The valence band averaged polarization is given by .

$$
P_{\sigma-}=\frac{I^{(1)} P^{(1)}+I^{(2)} P^{(2)}}{I^{(1)}+I^{(2)}}
$$

where $I$ is the pump rate from the indicated valence band. Equation (3.16) shows that the spin polarization is $P_{e^{-}}=-0.5$ regardless of the relative pump rates from the two valence bands. The significance of the siga on the polarization is to describe the spin direction relative to the angular momentum vector of the incident photon. A positive polarization means the spin is parallel to the angular momentum vector of the photon, while a negative polarization means the spin is anti-parallel. The $50 \%$ result is valid without the simplifying assumption of spherically symmetric constant energy surfaces, as shown in a calculation provided in Appendix A.

\section{Uniaxially Strained GaAs}

The results for spin polarization due to optical transitions from each of the two upper valence bands given in eqs. (3.11) and (3.12) are valid for the case of uniaxially strained GaAs as well as gon-strained GaAs. The angular dependence in these equations has a different meaning for the uniaxially strained case, though. As previously stated, the angle $\theta$ is measured between the photon direction and the quantization axis of the valence level wave functions. For strained GaAs, the quantization axis for the valence wave functions is along the effective uniaxial strain 
axis. The angle $\theta$ is therefore fixed and integration over all allowed transitions leaves the equations for polarization ((3.11), (3.12)) unchanged. When the optical pump beam direction is along the effective uniaxial strain axis, spin polarizations of $-100 \%$ and $+100 \%$ are obtained for transitions from the $\left\langle\frac{3}{2}, \frac{3}{2}\right\rangle$ and $\left|\frac{3}{2}, \frac{1}{2}\right\rangle$ states, respectively.

The valence level wave functions which were used to obtain the spin polarization relationships for strained GaAs are strictly valid only at $\Gamma$. For finite strain, nonzero electron momentum breaks the symmetry which is responsible for the wave functions at $\Gamma$ and causes mixing of the pure $J, m_{j}$ states. This mixing will affect the expected spin polarizations obtained by optical pumping. The effects of nonzero $\vec{k}$ on spin polarization will be considered at the end of this chapter.

\subsection{Luminescence Circular Polarization}

\subsubsection{Description}

In p-type GaAs, the thermal equilibrium density of electrons in the conduction band is extremely small compared to the valence band hole population. Therefore, the spin polarized conduction electrons produced by optical pumping are assumed to constitute the entire population of conduction electrons while the valence hole population is assumed to remain virtually unchanged. It is further assumed that hole spin depolarization and thermalization is rapid relative to radiative recombjnation times. Luminescence circular polarization is then the result of a well defined situation of radiative recombination of spin polarized electrons with unpolarized holes. 


\subsubsection{Calculation}

The radiative recombination of minority electrons in GaAs may be described by the same electric dipole interactions which describe photo-excitation. In a manner similar to the spin polarization calculation, a density operator of valence level states may be used to construct an interaction matrix which describes the photon helicity obtainable from radiative recombination. The density operator is given by eq. (3.5) and the basis it is to act witkin is given by

$$
\left.\left.\left.\left.\left|\mathbb{D}^{+}\right| m_{j}=1 / 2\right\rangle,\left|\mathbf{D}^{-}\right| m_{j}=1 / 2\right\rangle \text { or }\left|\mathbf{D}^{+}\right| m_{j}=-1 / 2\right\rangle,\left|\mathbf{D}^{-}\right| m_{j}=-1 / 2\right\rangle \text {. }
$$

The terms $\mathrm{D}^{+}$and $\mathrm{D}^{-}$are dipole operators corresponding to $\sigma^{+}$and $\sigma^{-}$radiation respectively. The operator/conduction state pairs shown in eq. (3.17) form bases for the possible radiative recombination. Choosing the basis which incorporates conduction states of $m_{j}=+1 / 2$, a luminescence helicity density matrix can be formed by

$$
f_{D, D^{\prime}}(\uparrow)=\sum_{n} N_{n}\left(m_{j}=1 / 2|\mathrm{D}| \Psi_{n}\right\rangle\left\langle\Psi_{\mathbf{n}}\left|\mathrm{D}^{\prime}\right| m_{j}=1 / 2\right\rangle .
$$

The subscripts $D$ and $D^{\prime}$ correspond to the two Dipole operators for circularly polarized light, and the term $N_{n}$ refers to the population density of holes for the valence state $\mathbb{\Psi}_{n}$.

The degree of polarization of luminescence is obtained from the matrix given in eq. (3.18) by

$$
\vec{P}_{\gamma}(\uparrow)=\frac{\operatorname{Tr}(f(\uparrow) \cdot \vec{\sigma})}{\operatorname{Tr}(f(\uparrow))},
$$

where the components of $\vec{\sigma}$ are identical to the Pauli spin matrices. The meaning 
of the various components of $\vec{P}_{\gamma}$ is somewhat different than for the case of electron epin polarization. Here, the $\hat{z}$ component of $\vec{P}_{\gamma}$ gives the degzee of circular polarization of the radiation while the $\hat{x}$ and $\hat{y}$ components give the degree of linear polarization in the $\hat{x}$ and $\hat{y}$ directions, respectively.

A separate luminescence helicity density matrix may be calculated for radjative transitions to each of the two $J=3 / 2$ valence bands. In order to calculate the matrices, a luminescence photon direction must be defined. The valence level states are expressed in terms of the coordinate system defined by the luminescence direction with the rotation matrices from eqs. (2.15) and (2.16). The conduction states, which are quantized along the original pump beam direction, must also be expressed in terms of the luminescence photon direction. This is accomplished with the rotation matrix given in eq. (2.15), but the angle $\theta$ involved in this rotation will be called $\omega$ for clarity. After calculating the luminescence helicity matrices, the luminescence circular polarizations originating from conduction $m_{j}=+1 / 2$ states are found to be

$$
P_{\gamma}^{(1)}(\uparrow)=\left(\frac{-2 \cos ^{2} \theta}{1+\cos ^{2} \theta}\right) \cos \omega
$$

and

$$
P_{\gamma}^{(2)}(\dagger)=\left(\frac{2-6 \sin ^{2} \theta}{2+3 \sin ^{2} \theta}\right) \cos \omega .
$$

The superscripts (1) and (2) jentify circulas polarizations from transitions to the $\left|\frac{3}{2}, \frac{3}{2}\right\rangle$ and $\left|\frac{3}{2}, \frac{1}{2}\right\rangle$ valence bands, respectively. The $\cos \omega$ term takes into account any difference in orientation between the conduction electron spin quantization axis and the luminescence photon direction. However, because the spin quantization axis lies along the pump beam direction in an optical pumping scheme, the angle 
$\omega$ is equivalent to the angle between the purnp photon and luminescence photon directions. The $\theta$ dependent terms in parentheses are identical to the electron polarization terms found in eqs. (3.11) and (3.12) and their components must be averaged over all allowed wave vectors $\vec{k}$ just as done for the spin polarization case. The results of such averaging are identical to those obtained for the spin polarization case. It is convenient to define these terms as recombination radiation coupling factors $P_{h}^{(n)}$ which are equivalent to the electron spin polarization factors $P_{\mathrm{e}^{-}}^{(n)}$. Equations (3.20) and (3.21) are then rewritten as

$$
P_{r}^{(1)}(\dagger)=P_{h}^{(1)} \cos \omega
$$

and

$$
P_{\gamma}^{(2)}(\uparrow)=P_{h}^{(2)} \cos \omega
$$

The luminescence polarization expected for a given luminescence photon energy $h \nu$ must be averaged over transitjons to both $J=3 / 2$ valence bands in a manner similar to eq. (3.16). Because eq. (3.3) showed that the ratio of photon absorption to photon emission is a term common to all valence bands, it follows that the recombination radiation coupling factor $P_{k}$ must be equivalent to the spin polarization term $P_{\mathrm{e}}$ - upon averaging over the valence bands.

When the luminescence circular polarizations aue to transitions from $m_{j}=$ $-1 / 2$ conduction states are calculated, it is found that

$$
P_{y}(\downarrow)=-P_{\gamma}(\uparrow)
$$

With this equation, a complete relationship for luminescence circular polarization may be written. Transitions from both conduction band spin states may con- 
tribute, and it is therefore necessary to express the polarization as an average of the calculated polarizations from each of the spin states.

$$
P_{\gamma}=\frac{N_{t} P_{\gamma}(\uparrow)+N_{\downarrow} P_{r}(\downarrow)}{N_{\uparrow}+N_{\downarrow}}
$$

Here, $N_{\uparrow}$ and $N_{4}$ are the population deasities of $m_{j}=+1 / 2$ and $m_{j}=-1 / 2$ conduction states respectively. Using eq. (3.4), the population densities can be expressed as

$$
N_{\uparrow}=\left(1+P_{e}-\right) \bar{N}, N_{+}=\left(1-P_{e^{-}}\right) \bar{N},
$$

where $\bar{N}$ is the average population density. Combining eqs. (3.25) and (3.26), the complete expression for luminescence circular polarization is given by

$$
P_{\gamma}=P_{e}-P_{h} \cos \omega
$$

\subsubsection{Spin Relaxation}

The luminescence polarization was shown to be proportional to the conduction band electron spin polarization. The value for spin polarization used in eqs. (3.26) and (3.27) was that expected from optical pumping with circularly polarized light. It did not include the possibility of a lower electron spin polarization due to spin relaxation processes which may occur before radiative recombination of the conduction electrons. There are many proposed mechanisms for spin relaxation in GaAs which include, but are not limited to, the following:[28][29]

- The D'yakonov-Perel' (DP) process arises because of the lack of an inversion center in III-V structures. The degeneracy of spin-up and spin-down 
conduction states is broken, allowing depolarization to occur in a manner analogous to spin precession about an effective magnetic fiejd.

- The Bir-Aronov-Pikus (BAP) process explains spin relaxation in terms of the electron-hole exchange interaction. The coulomb potential between an interacting electron and hole is spin dependent, resulting in a spin flip probability.

- The Elliott-Yafet (EY) process takes into account the mixing between conduction and valence states which is found within the $\vec{k} \cdot \vec{p}$ analysis. The possibility of spin disorientation during scattering processes exists because the conduction states are not pure spin states due to mixing with valence $m_{j}=1 / 2$ states.

- The Hyperfine interaction calculates the electron spin relaxation due to the interaction between oriented electron and randomly oriented nuclear spin states.

- Reabsorption of recombination radiation accounts for the effective dilution of the average electron spin population by reabsorption of luminescence with a low average circular polarization along the quantization axis. The effectiveness of this mechanism requires a sizable luminescence efficiency and an optically thick active layer for reabsorption.

For the case of highly doped $p$-type GaAs, the so called BAP process[30] is considered to be the dominant spin relaxation mechanism. The DP process is actually 
enhanced under uniaxial strain along certain crystal directions, but not for a uniaxial strain along [001].

The strength of a spin relaxation mechanism is characterized by an exponential spin decay time $\tau_{s}$. The relaxation factor for a particular $\tau_{s}$ is given by[29]

$$
R=\frac{\tau_{s}}{\tau_{*}+\tau_{L}}
$$

where $\tau_{L}$ is the exponential lifetime of a conduction electron. It is assumed that the spin relaxation time and minority carrier lifetime are independent of both pump and luminescence photon energy. Therefore, the relaxation factor $R$ is considered to be independent of photon energy and simpiy a constant $0<R<1$. When the average electron polarization at the time of radiative recombination is adjusted for spin relaxation and used in eq. (3.26), the luminescence polarization $P_{\gamma}$ becomes

$$
P_{\gamma}=P_{e}-P_{h} R \cos \omega,
$$

as given in the introduction.

\subsubsection{Final Expressions}

\section{Non-Strained GaAs}

For non-strained GaAs, both $P_{t}$ and $P_{h}$ are equal to -0.5 so that the luminescence polarization will be given by

$$
P_{\gamma}=0.25 \cos \omega
$$

For an experimental geometry with an approximately normal incjdence pump beam and luminescence collected from within a small cone about the surface normal, 
the angle $\omega$ is approximately $180^{\circ}$ and the luminescence polarization is given by $P_{\gamma} \approx-0.25$.

\section{Uniaxially Strained GaAs}

For an experimental geometry equivalent to that described for non-strained GaAs, the Iuminescence polarization is given by

$$
P_{\gamma}=-P_{e}-\left(h \nu_{i n}\right) P_{h}\left(h \nu_{p}\right) R,
$$

where $h \nu_{i n}$ and $h \nu_{p l}$ are the incident pump photon energy and the luminescence photon energy, respectively. It is worth noting that $P_{\varepsilon}-$ and $P_{h}$ bave the same energy dependence, where $P_{e}$-carries the pump photon energy dependetite and $P_{h}$ carries the luminescence photon energy dependence.

\subsection{Consideration of Non-Zero $\vec{k}$ Effects on Polarization}

As stated in Section 3.2.2, the wave functions and subsequent polarization calculations for the case of strained $\mathrm{GaAs}$ are valid only at the $\Gamma$ point. Away from $\Gamma$, when $\vec{k}$ has a non-zero value, the interaction matrix is no longer diagonal within the $J, m_{j}$ basis. Although we know of no published solution which simultaneously incorporates strain and electron momentum, we may make an estimate of the $\bar{k}$ dependent solutions and the resulting spin polarizations obtainable. The process involves three steps beginging with unitary rotations within the valence band $X Y Z$ basis. First, for a general wave vector $\vec{k}=k_{x} \hat{x}+k_{y} \hat{y}+k_{z} \hat{z}$, mixing of $Y$ states with like-spin $X$ and $Z$ states as seen in eq. (2.6) is removed by a $\phi$ 
rotation which effectively makes $k_{y}=0$. The second step is to apply a proper $\theta$ rotation, thereby eliminating mixing between like-spin $X$ and $Z$ states. At this point, the interaction matrix is diagonal in the $X Y Z$ basis, however the diagonal $X$ and $Y$ terms are not equivalent. The non-equivalence of these terms leads to mixing between between $m_{j}= \pm 3 / 2$ and $m_{j}=\mp 1 / 2$ states upon transformation to the $J, m_{j}$ basis. Fortunately, these states are not degenerate and the final step is the application of standard perturbation theory to remove the mixing. The mixing between $\left|\frac{3}{2}, \pm \frac{3}{2}\right\rangle$ and $\left|\frac{3}{2}, \mp \frac{1}{2}\right\rangle$ states is treated to first order while the mixing between $\left(\frac{3}{2}, \pm \frac{3}{2}\right)$ and $\left(\frac{1}{2}, \mp \frac{1}{2}\right\rangle$ states is ignored because it is much smaller due to the larger energy splitting between the states. All necessary effective mass band parameters for the calculation are obtained from referesce [25].

Once solutions are found with the described process, the expected spin palarization is calculated by creating spin density matrices. The spin density matrices will be given by

$$
f^{\left(1^{\prime}\right)}=\frac{1}{1+M^{2}}\left(f^{(1)}+M^{2} f^{(2)}\right)
$$

and

$$
f^{(2)}=\frac{1}{1+M^{2}}\left(f^{(2)}+M^{2} f^{(1)}\right),
$$

where $f^{(1)}$ and $f^{(3)}$ are given by eqs. (3.9) and (3.10). The term $M$ in eqs. (3.32) and (3.33) is simply the state mixing found from the first order perturbation process. The spin density matrices are dependent on the magnitude and direction of $\vec{k}$. The magnitude of $\vec{k}$ is related to the photon energy by assuming direct ( $\vec{k}$ conserving) transitions. The meaning of $\theta$ in the $f^{(1)}$ and $f^{(2)}$ terms is the angle between the guantization axis and the pump beam. For the case of a pump beam 
along [001], $\theta$ is taken as the rotation necessary to remove the mixing between $X$ and $Z$ and is also dependent on $\vec{k}$.

The expected $\vec{k}$ dependent spin polarizations associated with transitions from each of the valence bands are shown in Figure 3.1 for various photon energies. The photon energies are chosen to be greater than the valence band specific gap minimum by an amount $0.25 \times \delta_{s} 0.5 \times \delta_{*}$, etc..., where $\delta_{s}$ is the strain induced band splitting energy. The spin polarizations are plotted against the polar angle of $\vec{k}$ where the effective uniaxial strain axis lies along $\hat{z}$. From Figure 3.1 we find that the spin polarization for transitions from the $\left|\frac{3}{2}, \frac{3}{2}\right\rangle$ band, averaged with equal weighting over the solid angle $\Omega_{k}$, only decreases from 1 to 0.95 for photon energies from the $\left(\frac{\frac{8}{2}}{2}, \frac{3}{2}\right\rangle$ band edge up to the $\left(\frac{3}{2}, \frac{1}{2}\right\rangle$ band edge. 

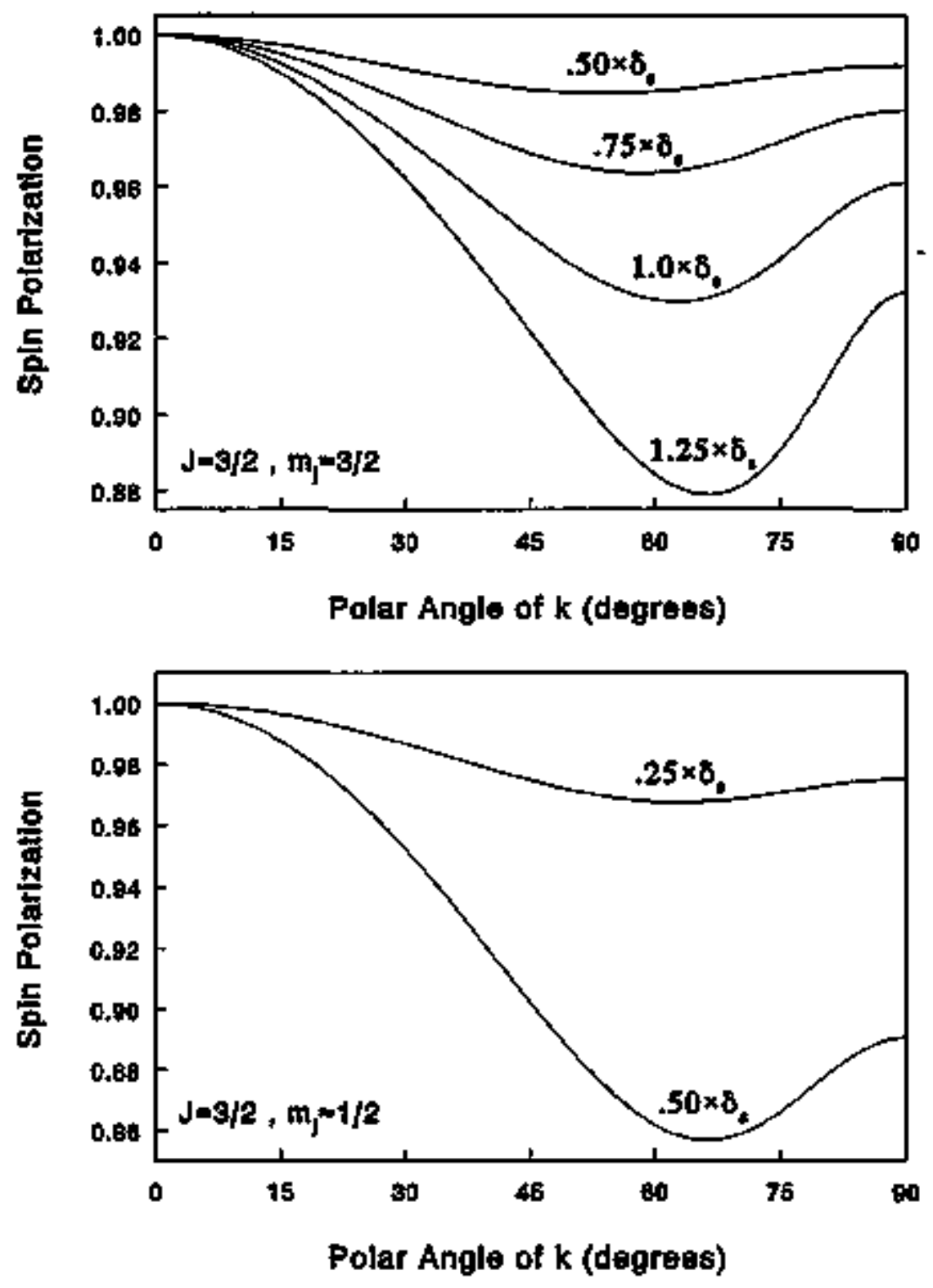

Figure 3.1: Spin polarization of conduction electrons photo-excited from the $\left|\frac{3}{2}, \frac{3}{2}\right\rangle$ and $\left|\frac{3}{2}, \frac{1}{2}\right\rangle$ valence bands plotted as a function of electron wave vector angle measared from the uniaxial strain axis. Each line corresponds to a different photon energy $-E_{\gamma}-E_{g a p}=0.50 \times \delta_{s}$ etc. - where $E_{\text {gap }}$ is valence band specific. 


\section{Chapter 4}

\section{Experimental Description}

\subsection{System Overview}

The experimental apparatus was designed for the study of polarized luminescence from optically pumped samples of GaAs photocathode structures. The pump source may be any one of several different lasers installed. These include a $\mathrm{CW}$ HeNe laser, a CW diode laser with either a $750 \mathrm{~nm}$ or $780 \mathrm{~nm}$ head and a CW tunable Ti-doped sapphire laser pumped with an Argon Ion laser. Laser polarization is controlled with a linear polarizer/quatter wave plate pair such that both linear and circular polarization states are possible. Luminescence is transported via an array of lenses and facussed onto the entrance slit of a double-spectrometer assembly. Luminescence polarizatjon is analyzed with a quarter wave plate/linear polarizer pair similar to the pump laser polarizer arrangement. The double-spectrometer is comprised of two spectrometers used in a negative dispersion arrangement. The dispersion of each spectrometer is different so that the pair still provides a net dispersion. The luminescence is recorded with a djode-array detector with roughly 700 active elements spanning an approximate wavelength range of $112 \mathrm{~nm}$. Samples may be mounted on a stage for room temperature measurements or in a double 
dewar system with a cold finger assembly capable of holding up to six samples. Temperatures as low as $\sim 8 \mathrm{~K}$ are accessjble with the dewar system. Both the dewar system arid the sample stage used for room temperature measurements have full linear $x y z$ translation capability for accurate sample alignment.

\subsection{Photocathode Description}

A total of nine different photocathode structures were used in this experiment. Seven of the samples were grown by the Spire Corporation[31] using Metal-OrganicChemical-Vapor-Deposition (MOCVD). The basic structure for each of these samples consists of the following. A $250 \mathrm{~nm}$ thick p-type GaAs buffer layer was grown on a vicinal (100) p-type GaAs substrate oriented two degrees toward the nearest [110] direction. One sample, \#2 in Table 4.1, used a substrate with less than $0.5^{\circ}$ miscut from [100]. After the GaAs layer, a. $2.5 \mu \mathrm{m}$ layer of $p$-type $\mathrm{GaAs}_{(1-x)} \mathrm{P}_{x}$ was grown with the phosphorus fraction $x$ increasing from zero to the target value $x_{0}$. This layer provides a transition from the lattice constant of GaAs to the smalles lattice constant of $\mathrm{GaAs}_{\left(1-x_{0}\right)} \mathrm{P}_{x_{0}}$ through the controlled introduction of misfit dislocations.[32][33] Next, $2.5 \mu \mathrm{m}$ of $p$-type GaAs(1-x) $\mathrm{P}_{ \pm}$was grown with the phosphorus fraction fixed at $z=x_{0}$. Finally, a thin layer of $p$-type GaAs was grown. This layer is intended to be in a state of strain due to pseudomorphic growth on the lattice mismatched 'substrate' of $\mathrm{GaAs}_{(1-x)} \mathrm{P}_{z}$ beneath. The thickness of the GaAs active layer varies from 100 to $500 \mathrm{~nm}$ for the different cathodes studied. All layers in these structures were $\mathrm{Zn}$ doped at a level of $4-6 \times 10^{18} / \mathrm{cm}^{3}$. A diagram of the Spire photocathode structure is shown in Figure 4.1. 


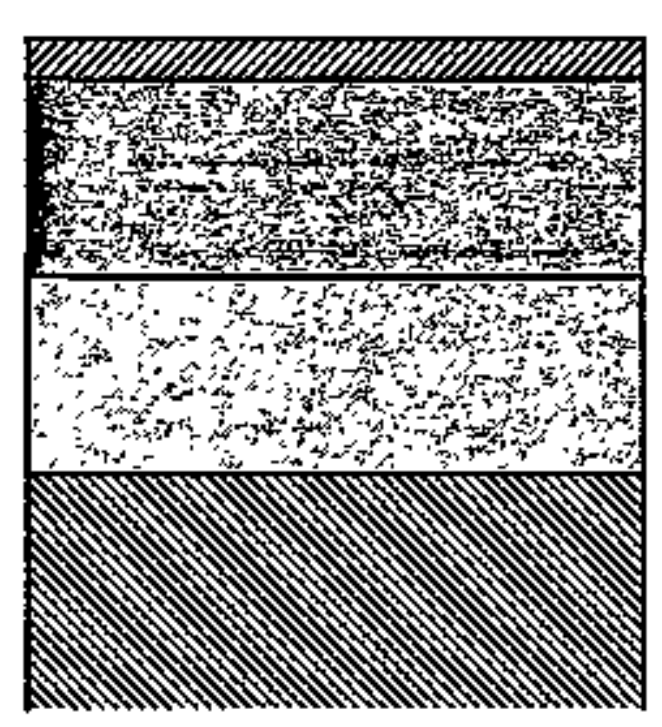

\author{
Strained GaAs \\ $t=100 \cdot 500 \mathrm{~nm}$ \\ GaAsP \\ P constant \\ $=2.5 \mu m$
}

GaAsP

$P$ graded

$\mathrm{t}=2.5 \mu \mathrm{mm}$

GaAs

Substrate

Figure 4.1: Structure of the GaAs/GaAs $\mathrm{s}_{(1-x)} P_{x}$ photocathodes grown by Spire Corporation.

In addition to the seven strained GaAs photocathodes grown by Spire, a nonstrained cathode was included in the study. This photocathode was grown by Molecular Beam Epitaxy (MBE) at the University of Ilinois in Urbana-Champaigz. The structure consists of a $200 \mathrm{~nm}$ strain-free active layer of GaAs grown on a 900 nIn $\mathrm{Al}_{, 30} \mathrm{Ga}_{70} \mathrm{As}$ layer which was itself grows on a GaAs substrate. The cathode is considered strain free because the $\mathrm{Al}_{.30} \mathrm{Ga}_{.70} \mathrm{As}$ lattice constant differs from that of GaAs by less than $0.05 \%$. All MBE layers of this cathode were Be doped to a level of $5 \times 10^{18} / \mathrm{cm}^{3}$. This cathode represents the lower bound for the range of active layer strains studied, namely zero.

The final photocathode used in this study was a hetero-epitaxially strained GaAs structure grown by Gas Phase MBE at the University of California at San Diego. This structure is similar in nature to the $\mathrm{GaAs}_{3} / \mathrm{GaAs}_{(3-x)} \mathrm{P}_{x}$ photocath- 
odes, except the substrate and grown layers are of different material. On a GaP

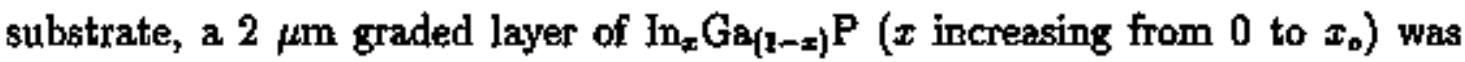
grown. Next, $2 \mu \mathrm{m}$ of $\mathrm{In}_{x_{0}} \mathrm{Ga}_{\left(1-\Phi_{0}\right)} \mathrm{P}$ was grown, followed by the $100 \mathrm{nIn}$ strained GaAs layer. All layers were Be doped at $4-6 \times 10^{18} / \mathrm{cm}^{3}$. This sample did not become available until most data had been collected for the other samples. It was included because it allowed for a check of any effects on the luminescence asymmetry data due to the necessary sample preparation procedures of the other photocathodes. The details of sample prepaxation and the difference between this photocathode and the other eight epitaxial layer photocathodes will be discussed in the following section.

Table 4.1 shows a list of the nine photocatiodes used in this study and jnformation such as active layer thickness, compasition of the strain-inducing buffer layer and the target strain for each active layer. When comparing luminescence data for the samples, two of the primary parameters of interest will be the active layer thickness and its state of strain. Characteristics of the layer immediately below the active layer will ideally only affect the luminescence data indirectly in the sense that these characteristics are related to the state of strain in the active layer. The degree of strain present in each of the photocathodes is dependent on both the initial lattice mismatch (provided by the GaAsP or InGaP layer) and on the degree of strain relaxation which has taken place. Therefore, the degree of strain in a photocathode is not accurately known simply from the buffer layer parameters and must be measured with X-ray diffraction techniques. The results of such measurements will be discussed in the next chapter. 
Buffer Layer

\begin{tabular}{cccc} 
Sample \# & $\mathrm{t}(\mathrm{nm})$ & (nominal) & $\epsilon^{\text {turget }}\left(\times 10^{-3}\right)$ \\
\hline 1 & 100 & $\mathrm{GaAs}_{.72} \mathrm{P}_{.28}$ & -10.0 \\
2 & 100 & $\mathrm{GaAs}_{.70} \mathrm{P}_{.30}$ & -10.7 \\
3 & 100 & $\mathrm{GaAs}_{.74} \mathrm{P}_{.25}$ & -9.3 \\
4 & 100 & $\mathrm{GaAs}_{.80} \mathrm{P}_{.20}$ & -7.1 \\
5 & 200 & $\mathrm{GaAs}_{.76} \mathrm{P}_{.24}$ & -8.6 \\
6 & 300 & $\mathrm{GaAs}_{.76} \mathrm{P}_{.24}$ & -8.6 \\
7 & 500 & $\mathrm{GaAs}_{.76} \mathrm{P}_{.24}$ & -8.6 \\
8 & 200 & $\mathrm{Al}_{.30} \mathrm{Ga}_{.70} \mathrm{As}$ & 0.0 \\
9 & 100 & $\mathrm{In}_{.35} \mathrm{Ga}_{.65} \mathrm{P}$ & -10.0 \\
\hline
\end{tabular}

Table 4.1: Nominal parameters for the photocatbode samples included in the luminescence study. Parameters include active layer thickness ( $t$ ), buffer layer composition and active layer target strain $\left(\epsilon_{\|}^{\text {targef }}\right)$. 


\subsection{Sample Preparation}

\subsubsection{General Preparation}

The various photocathode structures used in this study were grown on large (2-3 inch) diameter wafers. An exception is the non-strained epitaxial GaAs sample (\#8 from Table 4.1) which was grown on a $12 \mathrm{~mm}$ diameter wafer - the desired size for a single photocathode. All of the other photocathode materials had to be cut to a size suitable for the luminescence and photo-emission experiments.

Prior to cutting, materials were prepared for storage. Because GaAs oxidizes in air, it was necessary to protect the active layer surface of the epitaxial photocatbodes. The protection methods used for each photocathode material are listed below.

- Photocathodes grown by the Spire corporation were oxidized in a controllable manner to protect the surface from continual oxidation. This was accomplished with anodic oxidation in a weak solution of $\mathrm{H}_{3} \mathrm{PO}_{4}$-[34] Typically, an oxide layer of approximately $100 \AA$ was created.

- The photocathode structure of strajned $\mathrm{GaAs}$ on $\mathrm{In}_{x} \mathrm{Ga}_{(1-x)} \mathrm{P}$ was protected in the same manner as above.

- The non-strained epitaxial GaAs photocatbode was protected by an overcoating of elemental $\mathrm{Sb}$ deposited at the completion of growth. The $\mathrm{Sb}$ effectively protects the active layer surface from oxidation.[35] This sample required no further protection upon arrival at SLAC. 
After the photocathode materials were protected, they were waxed with paraffin to a piece of glass, so that the active layer was against the glass. They were then stored until cut for use.

The photocathode materials were cut with a diamond impregnated wire saw while still waxed to a piece of glass so that the front surface was well protected. For photo-enission experiments, a $12 \mathrm{~mm}$ diameter puck was cut from the wafer. This was also a suitable size for luminescence experiments. The circular shape of the cut was accomplished by waxing a geared cylinder to the 'backside' of the photocathode. The cylinder served as both a template for the wire saw and as a means of slowly rotating the wafer as it was cut.

After a photocathode sample bad been cut from the wafer, it was removed from the glass and cleaned with a cycle of boiling solvents. The standard cycle of solvents included trichloroethane, acetone and methanol, respectively. The sample was also subjected to ultrasound during the cleaning process.

The final step involved with the general preparation of a photocathode was to remove the protective oxide layer if it had one. This was done by simply rinsing the sample in ammonium bydroxide to dissolve the oxide layer.[34] If the cathode had a protective layer of elemental Sb (or As), the layer was not removed until the in situ heat cleaning performed during the cathode activation process of an electron photo-emissjon experiment. In this luminescence study, the photocathode which had a protective layer of elemental $\mathrm{Sb}$ was used for luminescence studies only after it had been used in a prior photo-emission experiment. 


\subsubsection{Preparation for Luminescence Experiments}

The samples ased in this study were grown as functional photocathode structures, which provided the opportunity to compare luminescence data with photo-emission data. Although, in principle, a luminescence study of functional photocathodes was preferred over the use of structures grown specifically for luminescence experiments, it proved quite difficult in practice. The epitaxial GaAs photocathodes (both strained and non-strained) were all grown on GaAs substrates with the single exception of sample \#9 from Table 4.1. It was quickly realized that when optically pumping one of these samples near band edge, the GaAs substrate was also pumped because the larger band gap buffer did not absorb the remaining pump beam. Recombization luminescence from the substrate occurs over the sane wavelength region as that from the GaAs active layer of interest, and it was found to be as much as several hundred times greater than that from the active layer.

The relatively low luminescence intensity seen from a thin GaAs active layer is attributable to two distinct factors. First, the active layer of the sample is very thin $(.1-.5 \mu \mathrm{m})$, so that, under the condition of near band edge optical pumping, only a small fraction of the incident pump beam was absorbed within the layer. Secondly, the possibility existed for non-radiative recombination of the optically pumped electrons at both the GaAs/air and GaAs/buffer layer jnterfaces. The parameter used to characterize this type of recombination is the surface recombjnation velocity. Other 'bulk' non-radiative recombination channels may also limit the Juminescence intensity, but the interface recombination is most likely a much stronger effect considering the active layer thicknesses of the samples ured in this 
study. We may make an estimate of the interface and thickness effects on luminescence efficiency by using the steady state rate equation given by Duggan and Scott[36] which takes into account non-radiative recombination at both surfaces. The steady-state excess minority-carrier concentration, $N$, is given as

$$
N=L\left[n_{2}\left(e^{d / L}-I\right)-n_{1}\left(e^{-d / L}-1\right)\right]-\frac{n_{3}}{\alpha}\left(e^{-\alpha d}-1\right),
$$

where

$$
\begin{gathered}
n_{3}=\frac{I_{o} \tau \alpha(1-R)}{\left(1-\alpha^{2} L^{2}\right)} \\
n_{2}=n_{3}\left(\frac{\left(S_{1}+\alpha D\right)\left(S_{2}-D / L\right) e^{-d / L}-\left(S_{1}+D / L\right)\left(S_{2}-\alpha D\right) e^{-\alpha d}}{\left(S_{1}+D / L\right)\left(S_{2}+D / L\right) e^{d / L}-\left(S_{1}-D / L\right)\left(S_{2}-D / L\right) e^{-d / L}}\right) \\
n_{1}=-n_{3}\left(\frac{\left(S_{1}+\alpha D\right)\left(S_{2}+D / L\right) e^{d / L}-\left(S_{1}-D / L\right)\left(S_{2}-\alpha D\right) e^{-\alpha d}}{\left(S_{1}+D / L\right)\left(S_{2}+D / L\right) e^{d / L}-\left(S_{1}-D / L\right)\left(S_{2}-D / L\right) e^{-d / L}}\right) n_{n}
\end{gathered}
$$

Here, $\tau$ is the bulk minority-carrier lifetime, $R$ is the reflectivity of the sample, $I_{a}$ is the incident pump photon fux density, $d$ is the active layer thickness, $D$ is the diffusion coefficient for the electrons, $S_{1}, S_{2}$ are the surface recombination velocities, $L$ is the diffusion length, and $\alpha$ is the absorption coefficient for the incident light. In order to calculate a minority carrier concentration ratjo between the case of a bulk material and an epitaxial active layer, we use material parameter values of $D=50 \mathrm{~cm}^{2} / \mathrm{sec}, L=1 \mu \mathrm{m}$, and $S_{1}=S_{2}=5 \times 10^{5} \mathrm{~cm} / \mathrm{sec}$. The absorption coefficient is taken as $\alpha=0.5 / \mu \mathrm{m}$ to represent absorption near band edge, and the active layer thicknesses are taken as $d=.1 \mu \mathrm{m}$ and $d=600 \mu \mathrm{m}$ for epitaxial and bulk photocathodes, respectively. With these values for the equation parameters, we find that a ratio of $353 \times$ is expected for the relative bulk/epitaxial PL intensities.

It should be mentioned that attempts were made to 'passify' the surface of the cathode samples in the hope of achieving large enough luminescence intensities 
with which to work. Many authors have described methods for passifying the surface of $\mathrm{GaAs}$ by removing native surface oxides and terminating surface bonds with alternative chemical makeups.[37]-[41] We tried two different techniques and did achieve modest enhancements in the luminescence efficiency of the samples. The first technique tried was anodic deposition of sulfur on the surface.[40] Here, the sample was anodized in a solution of $\left(\mathrm{NH}_{4}\right)_{2} \mathrm{~S}$ at a potential on the order of tens of volts. With this technique, luminescence enhancements of approximately a factor of two were observed for a bulk GaAs material pumped with a $633 \mathrm{~nm}$ laser. However, the epitaxial samples did not show improvement. In fact, it appears that the process oxidized all of or a large portion of the active layer on these samples. The second method attempted was application of I Molar $\mathrm{Na}_{2} \mathrm{~S} \cdot 9 \mathrm{H}_{2} \mathrm{O}$ to the sample surface.[41] This technique showed the similar factor of two enhancement in luminescence efficiency for bulk GaAs. Enbancements in the luminescence efficiency of epitaxial samples were also observed. A $300 \mathrm{~nm}$ active layer strained GaAs photocathode sample showed approximately a 3 fold enharcement, while a sample of $200 \mathrm{~nm}$ non-strained GaAs showed an apparent enhancement factor of 10 to 20 times.

\section{Substrate Etching}

The problem of unwanted luminescence from the GaAs substrate of the epitaxial photocathodes was overcome by removing the substrate from beneath an area of the photocathode active layer. This was done with a light-controlled wet chemical etching technique which we developed for our specific purpose. The substrates of 


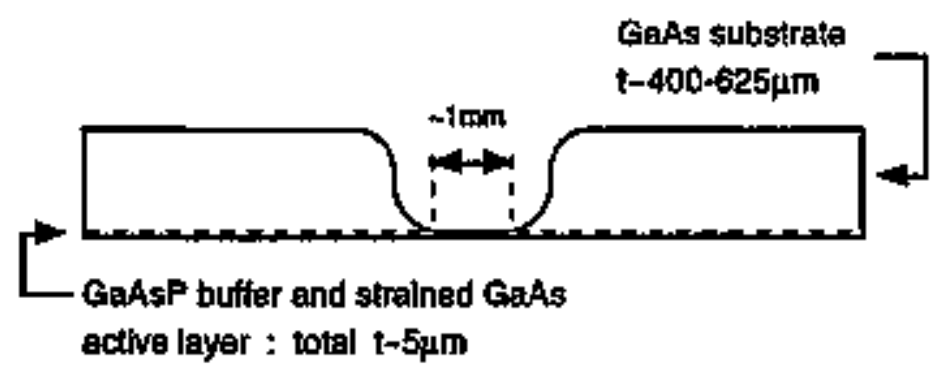

Figare 4.2: Schematic Profile of an etched $\mathrm{GaAs} / \mathrm{GaAs}_{(1-x)} P_{x=}$ photocathode. An area of approximately $1 \mathrm{~mm}^{2}$ devoid of GaAs substrate material was typically achieved.

these cathodes were 400-625 $\mu \mathrm{m}$ thick. A region was etched on the 'backside' of the substrate all of the way through to the substrate/buffer interface. Typically, a roughly circular area of $\approx 1 \mathrm{~mm}$ diameter was created which was completely devoid of any GaAs substrate. Figure 4.2 shows schematically a profile of an etched photocathode of strained GaAs on $\mathrm{GaAs}_{(1-x)} \mathrm{P}_{x-}$.

There exist many different etching techniques for III-V compounds, several of which are designed to express an etching selectivity of one material over another. Selectivity between $n$-type and $p$-type $\mathrm{GaAs}$ is possible with the proper wet chemjcal solution.[42] Selective etching between GaAs and AlGaAs is also possible with the proper solutions.[43] Selectivity between GaAs and GaAsP has been demonstrated with a photochemical dry-etching technique.[44] The type of selectivity needed for the purposes of this study was the preferential etching of p-type GaAs relative to $p$-type GaAsP (or, for sample \#9, p-type $\mathrm{Al}_{3} \mathrm{Ga}_{7} \mathrm{As}$ ). The only promising method found in the literature was the photochemical dry-etching technique mentioned. Unfortunately, this is a fairly complex technique which would likely involve a substantial investment of equipment and time before providing useful 
results. Therefore, a method of achieving the necessary etch characteristjcs in a wet-etching arrangement was pursued.

Although the successful removal of several hundred microns of GaAs from a 1 $\mathrm{mm}$ area leaving a self-supporting layer of $\leq 5 \mu \mathrm{m}$ is quite impressive, the etching technique itself is fairly simple. The light-controlled nature of the-process is not new. It is known that photo-generated minority carrier accumulation at an etching surface can effect the chemical reaction rates.[45]-[47] When GaAs is optically pumped, an excess minority carrier population is generated. These minority carriers can diffuse to a strface where they will affect the charge distribution of the surface depletion region and surface states. For the case of p-type GaAs in an acidic etching solution, minority carrier diffusion to the etch surface tends to slow or stop the etching process, while it enhances the etching of n-type GaAs.[48] We developed an etching scheme in which the samples were illuminated from the protected active layer side while etching occurred from the substrate side. When the etch surface reached the GaAsP graded phosphorus layer, it was automatjcally suppressed by the photo-generated minotity carriers. While the illumination stopped the etch, jt also provided a visible signal that the etch was complete be cause the region devoid of GaAs substrate transmitted an easily observable amount of the red (long wavelength) portion of the etch-controlling illumination.

The solution used for etching was 8:1:1 $\mathrm{H}_{2} \mathrm{O}_{2}: \mathrm{H}_{2} \mathrm{SO}_{4}: \mathrm{H}_{2} \mathrm{O}$ at room tenperature. With this solution, the p-type GaAs was etched at a rate of $6.8 \mu \mathrm{m}$ per misute. Samples were prepared for etching by waxing thern to a thin cover-glass with the actjve layer against the glass. A coating of an etch resistant material called 
Coverlag[49] was then applied to the exposed surface of the sample with a small brush. An area of approximately $2 \mathrm{~mm}$ in diasneter was left unprotected to serve as the etch site. The thin layer of wax between photocathode surface and the cover-glass was visibly transparent such that it would not inhibit illumination of the sample. The sample and cover-glass, ready for etching, were then placed in a flat-bottomed quartz container with the exposed portion of the substrate facing up. Lateral movement of the sample within the contajner was limited by the container size, ensuring proper sample position for illumination. Ten to twenty milli-liters of the etch solution was freshly prepared for each sample and some fraction of this was used in the quartz container at any given time.

The eptire etching apparatus was erected on a ring stand as shown in Figure 4.3. The light source was a tungsten filament projector lamp. A cutofi filter, which passed only wavelengths shorter than $\lambda \sim 700 \mathrm{~nm}$, was placed between the light source and the sample. This allowed only photons of energies large enough to be absorbed in the $\operatorname{GaAs}_{(1-x)} P_{x}$ layers to reach the sample. If photons of energy less than the bandgap of the $\mathrm{GaAs}_{\{1-x)} \mathrm{P}_{x}$ were allowed to illuminate the sample, they could reach the substrate and create a minority carrier density which might stop the etch before the entire substrate was removed. An iris with a diameter of approximately $4 \mathrm{~mm}$ was also placed between the light source and the sample simply to block light which was not contributing to the etch process. The measured light intensity at the approximate sample position was in the range of $1-3 \mathrm{~W} / \mathrm{cm}^{2}$. It was clear that a higher light intensity was necessary for the $500 \mathrm{~nm}$ strained GaAs active layer sample as compared to the $100 \mathrm{~nm}$ active layer samples. This 


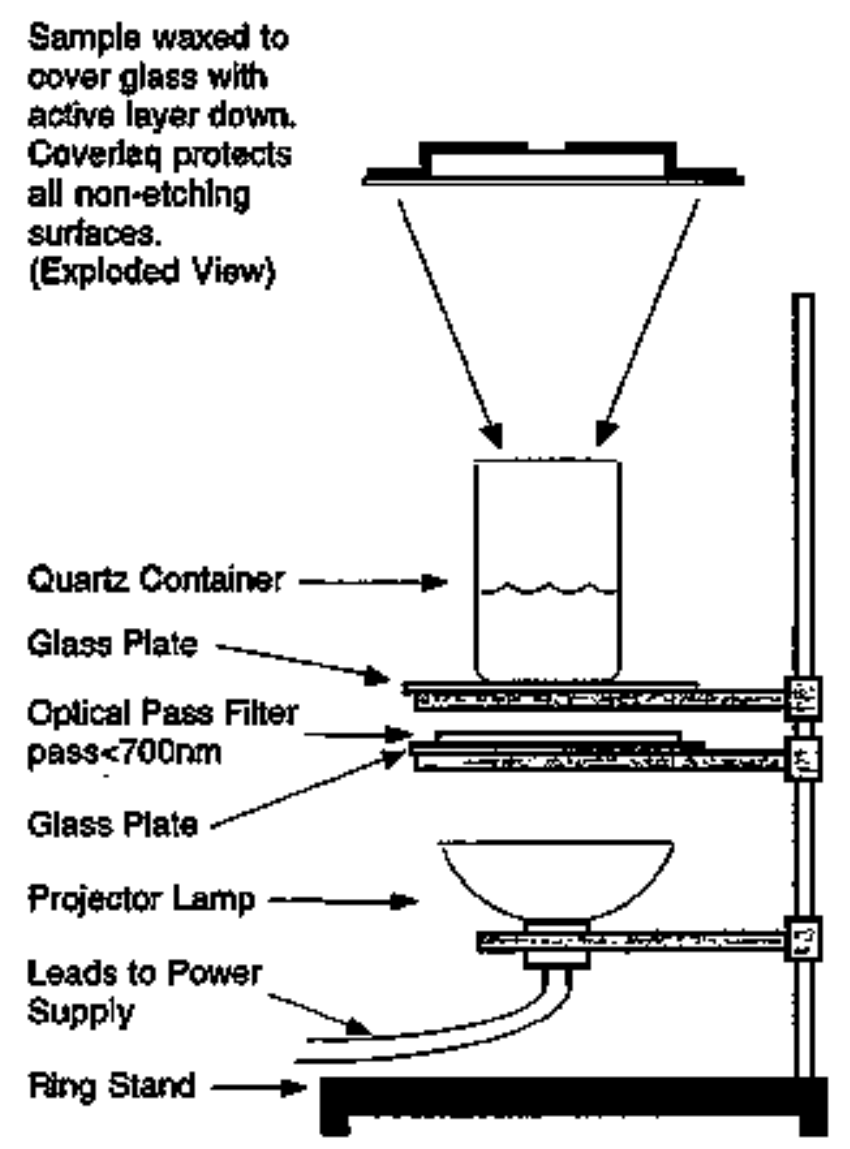

Figure 4.3: Diagram of the apparatus used to etch the photocathode samples. The system and technique are described in the text.

was due to the absorption of some of the incident light in the active layer which does not contribute to the minority carrier density within the $\operatorname{GaAs}_{(\mathbf{y}-\mathbf{x})} \mathrm{P}_{x}$ layer. When a sample etch was near completion, a small spot of transmitted red light appeared in the thinned region. The area of this spot would grow over the next several tens of seconds and when it appeared to be large enough (approximately $1 \mathrm{~mm}$ in diameter) the etch was stopped by adding a large amount of de-jonized water to the quartz container. 
After etching was complete, the samples were thoroughly rinsed in de-ionized water to ensure complete removal of the etchant. Next, the protective Coverlaq was removed from the sample. This was most easily accomplished by carefully peeling the material off of the sample substrate with a razor blade. Finally, the sample was placed in hot (but not boiling) trichloroethane to dissolve the wax which held it to the cover-glass. The sample was held in a pair of tweezers and cleaned in hot trichloroethane followed by acetone and then methanol. Photoluminescence measurements taken by optically pumping and viewing from the substrate side of the thinned region showed no detectable level of luminescence attributable to the GaAs substrate, indicating that the entire GaAs substrate was indeed removed.

All of the strained $\mathrm{GaAs} / \mathrm{GaAs}_{(1-x)} \mathrm{P}_{x}$ photocathode samples were etched with this light-controlled etch technique. It was also necessary to etch the non-strained epitaxial GaAs photocathode, grown with a $900 \mathrm{~nm}$ thick buffer layer of $\mathrm{Al}_{3} \mathrm{Ga}_{3} \mathrm{As}$. For this sample, a region had to be tbinned to a thickness of approximately $1 \mu \mathrm{m}$. The same light-controlled etch technique was used but, due to the thinness of the $\mathrm{Al}_{r 3} \mathrm{Ga}_{4} \mathrm{As}$ buffer layer, it was quite difficult to obtain a satisfactory etch. However, after several attenpts, a sample was successfully thinned over an area smaller than typical but still large enough for use.

\section{Application of Colloidal Graphite to Thinned Region}

After successful removal of the GaAs substrate, photocathode samples could be optically pumped and luminescence observed from the epitaxial layer. It was discovered though, that the relative laminescence intensity from the thinned region 
was greater than that from a non-thinned region of the sample. Also, in the thimned region, the circular polarization of the luminescence was lower than that from the non-thinned region. This effect was due to the reflective nature of the two surfaces of the thinned region. When the isotropic luminescence is emitted in a non-thinned sample, only the luminescence within an escape cone of an approximate $16^{\circ}$ half angle bas an opportunity to exit. The rest of the luminescence is already moving backwards or is reflected backwards and absorbed in the GaAs substrate. However, when the substrate is removed and replaced with a GaAsP/air interface, the large fraction of luminescence which is normally absorbed by the substrate can undergo multiple internal reflections until it escapes from one of the two surfaces or is absorbed. The internally refecting luminescence may ultimately escape because the front and rear surfaces are not optically flat.

This problem was solved by applying a coating of colloidal graphite (DAG)[50] to the back surface of a thinned sample. A single drop is applied to the back surface of the thinned region of a photocathode and allowed to dry in air. The proposed function of the DAG is to reduce or eliminate the possibility for multiple internal reflections of the luminescence. A sample of strained $\mathrm{GaAs}$ on $\mathrm{GaAs}(1-x) \mathrm{P}_{x}$ with the substrate removed was studied before and after the application of DAG to the thinned region. The luminescence measurements were made with the sarnple cooled to $78 \mathrm{~K}$ because the effects of multiply reflected luminescence were observed to be greater at this temperature. This is assumed to be due to reduced selfabsorption of the luminescence within the thinned layer at lower temperatures. With no DAG applied to the sample, there was an approximate $800 \%$ enhascement 
of the active layer Juminescence intensity in the thinned region relative to the nonthinned region. The circular polarization of the luminescence, under circularly polarized pump conditions, was correspondingly reduced in the thinned region relative to the non-thinned region by an approximate factor of 5.6. After applying DAG to the back surface of the thinned region, we found that the luminescence intensity from the thinmed region was enhanced by no more than $50 \%$ relative to the non-thinned region. The circular polarization of the luminescence also improved to a level in agreement with the polarization from the non-thinned region. These results were satisfactory, and DAG was applied to all of the etched samples.

The luminescence behavior of a photocathode which has had the GaAs substrate removed and effectively replaced with a coating of DAG may not be equivalent to the behavior from a photocathode which is not modified. For instance, it is possible that the DAG on a thinned sample does not inhibit internal reflection sufficient\}y, so that circular polarization measurements are affected. Fortunately, during the luminescence study, a photocathode grown on a GaP substrate was made available. This photocathode is sample \#9 (Table 4.1), described in Section 4.2 of this cbapter. Because the band gaps of both the substrate and buffer layer are much larger (several bundred meV) than that of the strained GaAs active layer, the photocathode may be optically pumped near band edge and only the active layer enits luminescence. This sample, therefore, did not have to be thinned. DAG was applied to the back surface of the substrate, however, so that internal reflection of luminescence would be suppressed. The DAG also prevented the pump beam from reflecting from the rear of the substrate or from exiting the 
substrate and scattering off of the sample mount.

\subsection{Cryostat and Sample Stage}

A double dewar system with a cold finger assembly provided a means to cool samples to temperatures as low as $\sim 8 \mathrm{~K}$. The vacuum level of the dewar system was approximately $10^{-6}$ Torr at room temperature and $10^{-8}$ Torr at $8 \mathrm{~K}$ and mainlained with an ion pump rated at 8 liter/sec. The system had no type of heating element or controlled gas flow incorporated for temperature control, so it was used at only liquid $\mathrm{N}_{2}$ and liquio He temperatures. The cold finger was in direct thermal contact with the base of the inner dewar which held the cooling liquid. The outer dewar, filled with liquid $\mathrm{N}_{2}$, was used as a thermal jacket. A shroud surrounding the cold finger was attached to the outer dewar in order to minimize thermal radiation to the cold finger. Only a small window faced the Juminescence optics line so that a cooled sarnple could be optically pumped and luminescence observed.

The temperature of the cold finger was monitored with two temperature calibrated diodes, model CY7-Cu-4, from Omega Engineering, Inc.[51] The quoted uncertainty for the diodes is $\pm 1 \mathrm{~K}$ in the range of 2-100 $\mathrm{K}$. The diodes were mounted on opposite sides of the cold finger and read within $1 \mathrm{~K}$ of each other at reduced temperature. When the inner dewar was filled with liquid $\mathrm{N}_{2}$, the diodes gave a temperature reading of $78 \mathrm{~K}$ which is within one degree of the normal boiling point for Nitrogen. The temperature readings during liquid He filts were also within $1 \mathrm{~K}$ of each other. However, depending upon the position of the rotatable 
cold finger, the readings varied from $\approx 8 \mathrm{~K}$ to $\approx 13 \mathrm{~K}$. This is believed to be due to variation in the quality of the thermal contact of the cold finger to the base of the dewar. The temperature for all measurements acquired during liquid He fills is referred to as $T \approx 12 \mathrm{~K}$ for convenience, with the tunderstanding that this number encompasses a range of $\sim 8-13 \mathrm{~K}$.

The cold finger and shroud, which hung below the dewars, were surrounded by a Pyrex cylinder with O-ring seals to stajnless steel flanges at each end. The Pyrex cylinder acted as a window for the incoming pump beam and exiting luminescence, so that care was taken to minimize any stress birefringence present. However, most data was taken at room temperature and samples were not mounted in the dewar, thus precluding concerns of the effects of the window. Both the room temperature mount (outside of dewar) and the dewar assembly were mounted on a translation stage rigidly attached to the luminescence optics bench. The stage provided full three dimensional linear translation capability so that samples could be accurately aligned without the need for any optical component adjustments.

\subsection{Laser System}

Although several lasers were available for the optical pumping of samples, nearly all of the luminescence data was obtained while pumping with the tunable Tidoped sapphire laser, model 3900s from Spectra Physics,[52] which is continuously tunable over wavelengths from $700 \mathrm{~nm}$ to $1 \mu \mathrm{m}$. A chilled water cooling system was used with the laser to remove excess beat from the sapphire crystal. The Ti:Sapphire laser was pumped with an argon jon laser, Spectra Physics model 
$171,[52]$ operated in a multi-line mode.

The Ti:sapphire laser can dejiver approximately 5 Watts of power when pumped with 20 Watts of power from the argon ion laser. The minimum lasing powers from this laser are, in fact, larger than the pumping requirements of the luminescence experiment. In order to deliver only the intensities needed to the luminescence samples, a $2 \%$ refiection pickoff/beam dump was constructed from a glass prism mounted in a black-anodized aluminum box. The lower intensity beam was then transported to the luminescence optics bench. Because the entire laser system was enclosed in secure boxes and beam pipes until emerging at the luminescence optics bench at a significantly reduced power, the dangers associated with the pump laser were greatly reduced.

\subsection{Optical System}

Figure 4,4 shows a top-view diagran of the optics bench layout for this experiment. The luminescence optics were enclosed in a box for the purpose of laser safety and extraneous toom light rejection. Mirror 4 in Figure 4.4 , a first surface protectedaluminum mirror, served to steer the selected laser beam down the incident beam optics line. In this optics line, an iris aided in the aljgnment of the laser beam, and a single lens focussed the beam after its polarization state was determined by a linear polarizer/quarter-wave plate pair. The Glan-Taylor polarizing prism and quarter-wave plate, consisting of a cleaved mica sheet cemented between two sheets of glass, were mounted in rotating click-lock devices which allowed for accurate $22.5^{\circ}$ incremental rotations. The excitation laser beam came into the sample at 
an angle of approximately $15^{\circ}$ to the surface normal, which faced directly down the luminescence optics line. The beam spot was focussed to approximately 100 $\mu \mathrm{In}$ at the sample.

A small cone of luminescence, subtending a solid angle of approximately 0.01 $\mathrm{Sr}$, was collected by the first lens pair of the luminescence optics line. The optics line, which imaged the point of sample excitation onto the entrance slit of a spectrometer, consisted of a series of lens elements, an iris, and a quarter-wave plate/linear polarizer pair. The net magnification of the lens array was about $2 x$. The linear polazizer and quarter-wave plate, a first-order multi-layer achromatic retarder designed for $\lambda / 4$ retardation centered at $850 \mathrm{~nm}$, were mounted in $22.5^{\circ}$ click-lock rotation devices similarly to the pump beam elements.

\subsection{Double Spectrometer}

The luminescence spectra were analyzed with a double spectrometer system consisting of two ISA model HR320 spectrometers[53] arranged in series. Each spectrometer has a $320 \mathrm{~mm}$ focal length with an acceptance of $f / 5.0$. The quoted light rejection for a single spectrometer is $10^{-5}$ at $1 \mathrm{~nm}$ from the laser line while using a 1200 groove/mm holographic grating. Because of very poor luminescence effciencies and high levels of scattered laser light due to sample surface roughness, it was found that the single spectrometer rejection level was insufficient for our measurements.

The use of two spectrometers in series provided adequate rejection of scattered laser light for the experiments. Coupling the spectrometers in a subtractive 


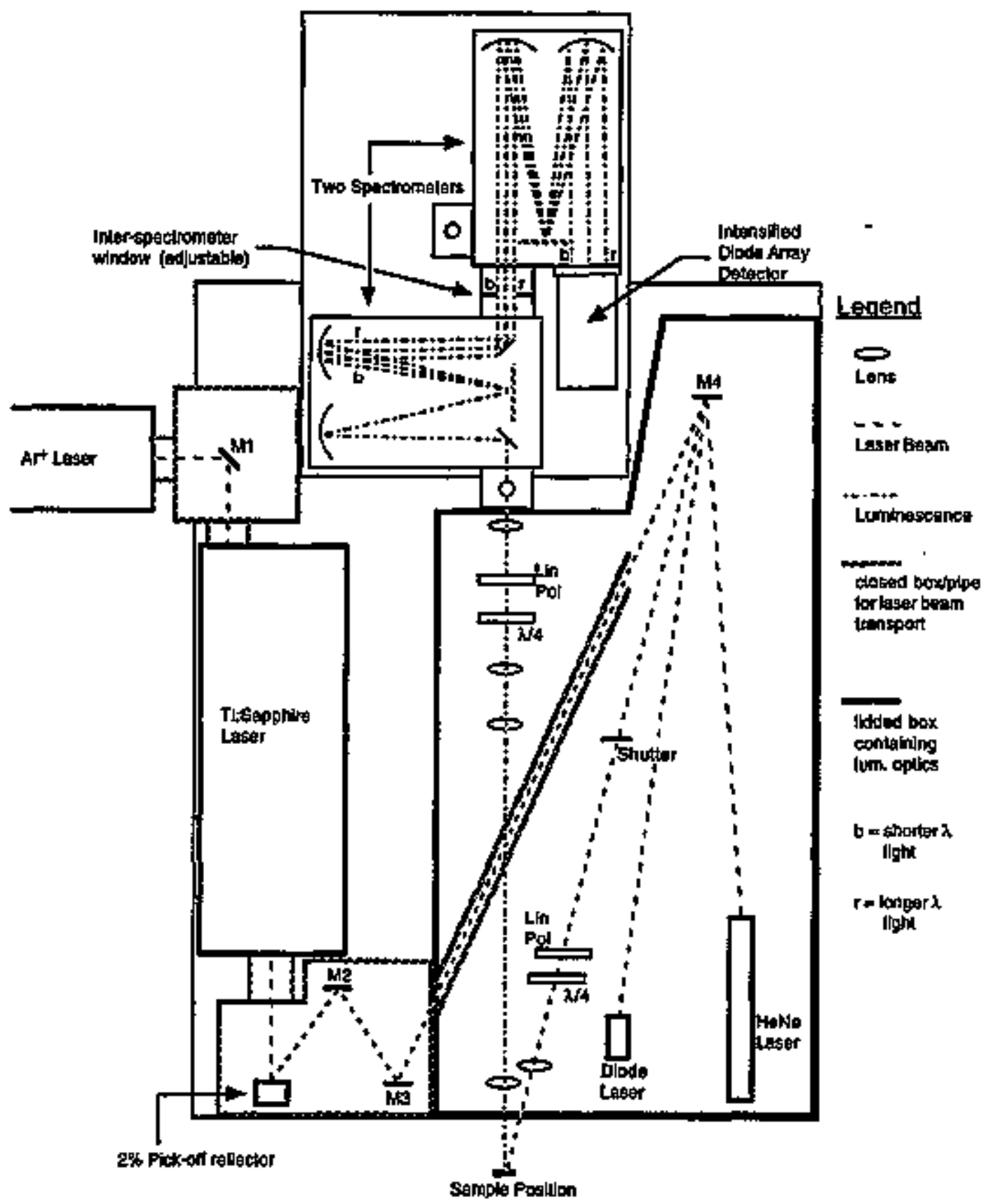

Figure 4.4: Top view diagram of luminescence bench and associated lasers. The system is described in the text. 
dispersion mode allowed for much higher rejection than an additive dispersion arrangement. Furthermore, by using gratings of different groove densities in each spectrometer, a net dispersion was maintajned. This was necessary in order to make use of an array-type detector which allowed for acquisition of a spectrum without scanning. The spectrometers were coupled as shown in Figure 4.4. The first spectrometer used a grating with a groove density of 150 groove/mm while the second spectrometer used a grating density of 600 groove $/ \mathrm{mm}$. An adjustable window mounted between the two spectiometers at the intermediate image plane allowed for selective passage of the luminescence into the second spectrometer while blocking the laser line. The window was a rectangular aperture approximately 7 mm wide and $2.3 \mathrm{~mm}$ high, and it's position along the dispersion direction was adjustable by micrometer movement so that the desired wavelength range could be passed to the second spectrometer.

The spectrometers were used in the following manner. The grating of the first spectrometer was adjusted to a position where wavelengths from approximately $560 \mathrm{~nm}$ to $940 \mathrm{~nm}$ could be passed into the second spectrometer without any further adjustments of the first spectrometer. Then, the desired wavelength range was inaged onto the array-type detector by proper adjustment of the interspectrometer window and grating position of the second spectrometer. The net dispersion of the system was $160 \mathrm{~nm} / \mathrm{inch}$ which corresponded to approximately $112 \mathrm{~nm}$ over the active zegion of the array-type detector. The vertical slit at the entrance to the first spectrometer was generally open to $200 \mu \mathrm{m}$ which corresponds to a resolution of $1.25 \mathrm{~nm}$, inuch narrower than any luminescence feature observed. 
When testing the double spectrometer performance, an improvernent of $10^{4}$ in the rejection of scattered laser light was found as compared to a singie spectrometer with a 600 groove $/ \mathrm{mm}$ grating. Further rejection wonld have been possible if the double-spectrometer were run in a traditional mode of subtractive dispersjon with identical grating and a narrow slit between spectrometers. This arrangement offers no net dispersion however, and luminescence spectra would have to be acquired in a scanning mode. The luminescence signals were sufficiently weak that data acquisition in scanning mode with a single detector was not practical.

\subsection{Detector and Data Acquisition System}

The detector used in this experiment was an Intensified Diode Array produced by Princeton Instroments.[54] The diode array is a 1024 element silicon array, one inch in length. Photons reaching the detector cause the emission of electrons from a multi-alkali transmission photocathode. The electrons are then accelerated and proximity focussed onto a micro-channel plate for amplification. The amplified signal excites a phosphor screen which is coupled to the diode array via a fiber optic grid. It is the photons from the phosphor which are detected and 'counted' by the elements of the diode array. The gain of the micro-channel plate is variable up to a level on the order of one count at the diode array for one electron created at the detector's photocathode. The detector has a usable range of approximately $370-920 \mathrm{~nm}$.

Dark current in the detector is greatest from the diode array itself but is bighly stable so that it can easily be subtracted out of data. The dark current is also 
greatly reduced with decreased operating temperature, so the detector is equipped with a thermoelectric cooler coupled to a water cooling system. During this study, the detector was run at a temperature of $-20^{\circ} \mathrm{C}$.

The detector was controlled by the Princeton Instruments ST120 Detector Controller[54], which was operated from a personal computer via a proprietary interface. The software which controlled the detector was also supplied by Princeton Instruments and provided control over such parameters as exposure time, number of spectra in a file, corresponding wavelength calibration file, and background subtraction file.

\subsection{System Calibrations}

\subsubsection{Wave Plate Calibration}

The quarter-wave retarders used in this experiment were both first order retarders, so that they offered near $\lambda / 4$ retardation over a fairly wide wavelength range. The ordinary (or extraordinary) axis of a retarder was found relative to the pass axis of the linear polarizing prism it was coupled with by placing it between a pair of crossed polarizers. When the axes of the retarder were coincident with the axes of the crossed polarizers, the extinction of a HeNe laser beam being passed through the elements was a maximum. The identity of each axis (ordinary or extraordinary) was then found by using the self-aligned quarter wave plate/linear polarjzer pair to analyze a partially circularly polarized bean of known helicity. The elliptically polarized beam was created with a linear polarizer followed by two total-internal-reflections in a glass prism. During total-internal-reflection, a phase 
shift of the reflected $s$ component relative to the $p$ component occurs. This phase shift may be calculated from the equations of reflectivity.[55] Furthermore, the phase shift ineurred is highly achromatic, so that with a white light source and a spectrometer analyzer, the wavelength dependence of the retarders was also easily determined.

\subsubsection{Spectrometer Wavelength Scale Calibration}

The wavelength scale of the double spectrometer systern was calibrated with the line spectrum from a Neon discharge lamp placed at the sample position of the optics bench. A separate calibration scale was obtained for each of several spectrometer grating positions (second spectrometer grating). The movement of the grating from spectrum to spectrum corresponded to $26.67 \mathrm{~nm}$ which is much smaller than the approximate $112 \mathrm{~nm}$ range of view of each spectrum. All of the calibrations were least-squares fits to a linear wavelength scale and the calibrations were stored as data files referred to by the data acquisition software.

\subsubsection{Spectral Response}

The overall spectral response of the luminescence optics, double spectroneter, and diode-array detector was determined for several grating positions (second spectrometer grating) similarly to the wavelength scale calibration. A tungsten ribbon filament lamp was placed at the sample position on the luminescence bench. The operating temperature of the lamp was calibrated as a function of current by the manufacturer, and was also checked with an optical pyrometer which has a calibrar tion traceable to the National Bureau of Standards. The radiation spectrum from 
the lamp was then calculated from the known surface temperature of the filament by assuming it was representative of black body radiation. The various spectra taken were thus tarned into spectral response correction data files and these correction files were subsequently used to correct luminescence spectra taken at the same spectrometer grating settings. It is worth noting that only the relative response was determined with this calibration. The absolute response at any given wavelength is not accurately known.

\subsubsection{Laser Wavelength Calibration}

The Ti:sapphire laser system was tunable by micrometer adjustment of a birefringent prism located within the resonating cavity of the laser. Two interchangeable mirror sets allowed lasing between roughly $700-850 \mathrm{~nm}$ and $850-1000 \mathrm{~nm}$, respectively. The wavelength scale relative to micrometer position was calibrated for each of the lasing ranges by observing the lasing wavelength with the caljbrated spectrometer system and recording the corresponding micrometer reading. The calibration obtained was used for wavelength tuning thereafter. Although it was possible for the calibration to change due to occasional 'tweaking' of the Ti:sapphire cavity mirrors, periodjc calibratjon checks indicated that such changes. were small and the wavelength uncertainty was estimated as $\pm 0.5 \mathrm{gm}$.

\subsection{Luminescence Data Acquisition Methods}

Accurate sample alignment and location of the center of the thinned region of samples was perfotmed prior to luminescence measurement. This was accomplished by 
monitoring the luminescence spectrum in real time while the sample was aligned. Luminescence measurements were generally performed with an appropriate bandpass filter in the incident beam optics and either a band-pass or long-wavelengthpass filter in the luminescence optics. Laser filters were installed 'upstream' of the polarizing optics and luminescence filters were installed 'downstream' of the polarizing optics. This insured that the filters did not interfere with polarization creation or analysis. The center of a sample's thinned region was relocated each time the pump beam filter was changed to insure optical pumping of the same region.

For luminescence polarization measurements, the pump power was monitored during data acquisition. The laser power was found to be highly stable with only a slight monotonic drift over full data acquisition times, so recording and correcting for pump power was unnecessary. Polarization measurements utilized all four pump/luminescence helicity combinations and the spectra were recorded consecutively in the order $\sigma^{+} / \sigma^{-}, \sigma^{+} / \sigma^{+}, \sigma^{-} / \sigma^{-}$, and $\sigma^{-} / \sigma^{+}$. Acquisition in this order minimized the small effect of the monotonic drift of laser power. The use of all four helicity combinations was found also to minimize false asymmetries due to beam steering effects associated with $\lambda / 4$ retarder rotation. The background files, which measure detector dark current, were recorded with each set of polarization spectra for low intensity data, while for higher intensity data it was necessary to record only a single background file for use with many subsequent polarization measurements. 


\subsection{X-ray Diffraction Measurement of Lattice Strain}

The degree of strain for the varjous cathodes was determined by X-ray diffraction measusement of the lattice spacing in the active layers. The measurement consists of acquiring a Bragg reflection peak from the active layer, the position of which is measured relative to the Bragg reflection peak for the non-strained substrate material. By acquiring an appropriate set of Bragg reflections, the precise state of strain of a photocathode may be determined.

The Bragg equation for reflection from atomic planes is

$$
2 d \sin \theta=n \lambda,
$$

where $d$ is the plane spacing, $\theta$ is half the reflection angle as measured from the incident beam direction, $\lambda$ is the $\mathrm{X}$-ray wavelength, and $n$ is the order of the Bragg reflection. For the most general case of a triclinic crystal, the plane spacing $d$ is related to the crystal lattice structure by[56]

$$
\begin{aligned}
& \frac{1}{d_{A k l}^{2}}=\frac{1}{1+2 \cos \alpha \cos \beta \cos \gamma-\cos ^{2} \alpha-\cos ^{2} \beta-\cos ^{2} \gamma} \\
& \times\left[\frac{h^{2} \sin ^{2} \theta}{a^{2}}+\frac{k^{2} \sin ^{2} \rho}{b^{2}}+\frac{\beta^{2} \sin ^{2} \alpha}{c^{2}}\right. \\
& +\frac{2 h k}{a b}(\cos \alpha \cos \beta-\cos \gamma) \\
& +\frac{2 k t}{b t}(\cos \beta \cos \gamma-\cos \alpha) \\
& \left.+\frac{2 h h}{c a}(\cos \gamma \cos \alpha-\cos \beta)\right\}
\end{aligned}
$$

The Miller indices $h, k$ and $l$ identify a specific reflection plane. The angles $\alpha, \beta$ and $\gamma$ are measured between the three primary axes of the crystal, and the lengths $a, b$ and $c$ are the lattice constants associated with each axis. GaAs is a cubic crystal so that, in a relaxed state, $\alpha=\beta=\gamma=90^{\circ}$ and $a=b=c=a_{0}$. Under the condition of a pure biaxial compression, as expected for pseudomorphic growth 
on a cubic crystal of slightly smaller laftice constant, the angles are preserved but the lattice constants change. The lattice constants in the plane of growth assume the value of the underlying substrate and are referred to as a constant in the direction orthogonal to the (001) growth plane is referred to as $a_{\perp}$. Equation (4.6) may be simplified for the case of tetragonal (or higher) symmetry to

$$
\frac{1}{d_{h \cdot j}^{2}}=\frac{h^{2}+k^{2}}{a_{\|}}+\frac{l^{2}}{a_{\perp}}
$$

such that $a_{\|}$and $a_{\perp}$ may be determined from two unrelated Bragg reflections. The strain (c) in each direction is defined as

$$
\epsilon_{\|(L)}=\frac{a_{\|(t)}-a_{o}}{a_{0}}
$$

Diffraction measurements for the strained layer samples were made with an X-ray diffractometer[57] which is part of a multi-user facility called the Center for Materials Research at Stanford University. Figuze 4.5 shows a diagram of the diffractometer. It has a $12 \mathrm{~kW}$ X-ray source producing $\mathrm{Cu}$ radiation lines. The X-ray beam may be passed through a Bartel's monochrometer, consisting of four (220) or (440) Bragg reflections from Ge crystals, to produce a highly monochromatic and collimated beam of $\mathrm{Cu}-K \alpha_{1}$ radiation. The monochrometer was used in the (220) configuration duxing measurements performed for this study. The goniometer has independent $\omega$ and $2 \theta$ movement capability and it is equipped with a Eulerian cradle sample stage to provide rotation capability along two more independent axes $\phi$ and $\psi$. The detector and assocjated optics are situated on the $2 \theta$ arm. The detector is a proportional counter with the final output given in counts/sec. Choices of detection optics include an open detector, a receiving slit 
aperture, a Bonse-Hart collimator or a parallel beam collimator. A recejving slit of $0.45 \mathrm{~mm}$ was used for the measurements performed for this study.

$\mathrm{X}$-ray diffraction measurements were made in the form of $\omega / 2 \theta$ scans. As shown in Figure 4.5, the sample angle relative to the incident beam is $\omega$ and the detector arm angle is $2 \theta$. During a $\omega / 2 \theta$ scan the angles are varied simultaneously, with $2 \theta$ sweeping at twice the rate of $\omega$, while the diffracted beam intensity is recorded. The incremental step of angular movement $\Delta 2 \theta$ was typically $.002^{\circ}$ to $.004^{\circ}$, which was sufficiently small to resolve the narrowest diftraction peale coming from the substrate. Scan times were chosen to provide sufficient counting statistics for the weak diffraction signals from the active layer. 

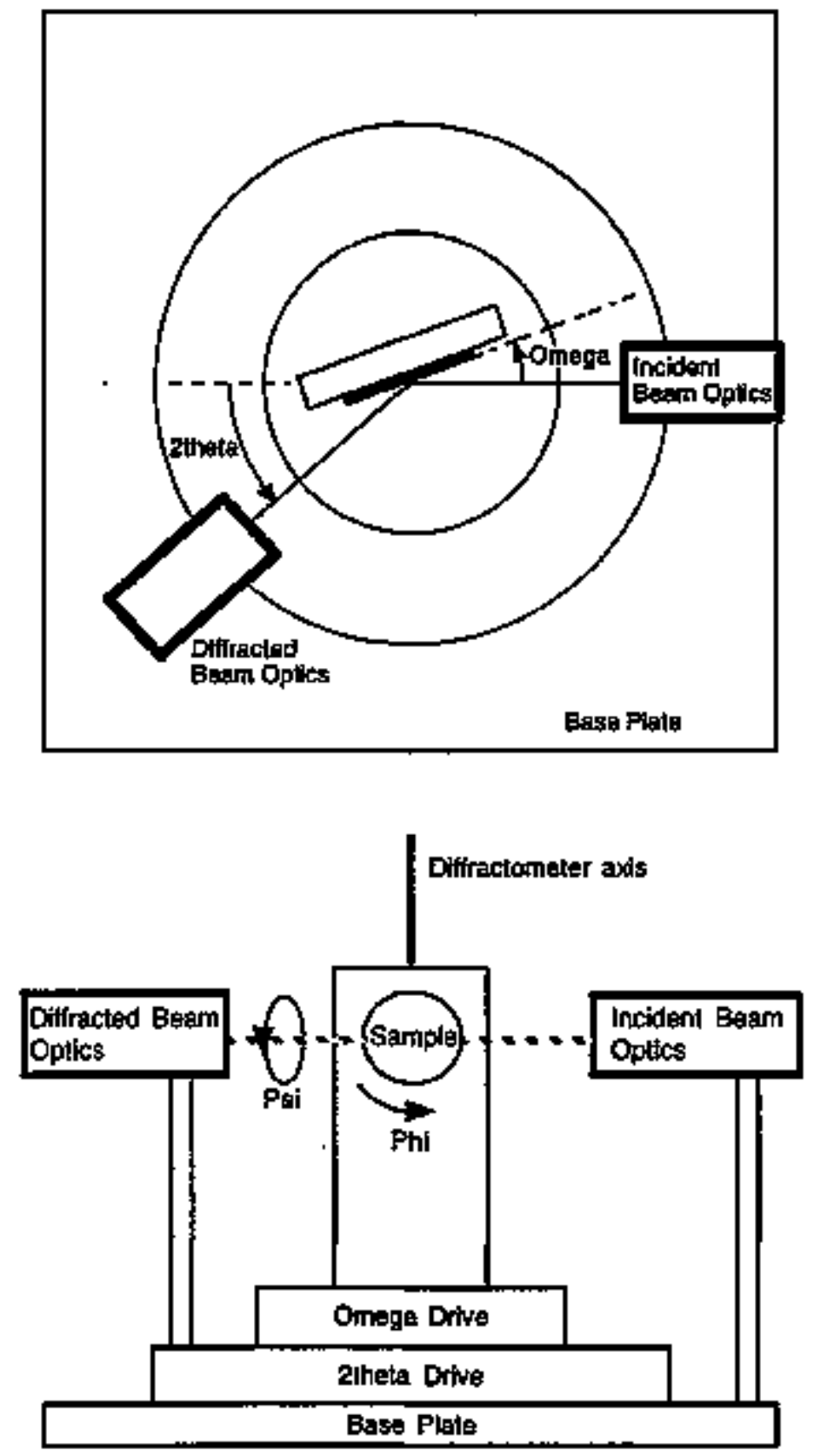

Figure 4.5: Plan view (top) and side view (bottom) diagrams of the X-ray diffractometer used to measure the lattice structure of the photocathode samples. The various controllable motions $(\omega, 2 \theta, \phi$ and $\psi)$ are shown. 


\section{Chapter 5}

\section{Data and Discussion}

\subsection{X-Ray Diffraction Measurements}

The photocathode structures studied in this work all incorporate an active layer of GaAs which is p-type doped to a level of approximately $N_{A}^{-}=46 \times 10^{18} / \mathrm{cm}^{3}$. Ideally, the only differences between the various photocathode active layers are thickness, degree of strain and degree of strain relaxation. The active layer thickness of a given photocathode is known reasonabiy well from the target thickness stated in the growth specifications because growth parameters are pre-calibrated. The crystalline state of the layers must, on the other hand, be measured by means of X-ray diffraction techniques.

$\mathrm{X}$-ray diffraction measurements were carried out on the eight strained layer photocathode samples included in this study. Sample number \#8 from Table 4.1 is comprised of a layer of GaAs Grown on $\mathrm{Al}_{3} \mathrm{Ga}_{7} \mathrm{As}$. The lattice spacings for these two layers are neazly identical so that no measurable strain will be present. Therefore, no X-ray diffraction messurements were performed for this sample.

The diffraction measurements were obtaised using the equipment and techniques described in Chapter 4 . The $0.45 \mathrm{~mm}$ detector slit corresponded to a $2 \theta$ 
acceptance of $0.15^{\circ}$, which is Inuch narrower than the $2 \theta$ width of the Bragg peaks from the highly mosaic active and buffer layers of the samples. This detector geometry provided both a sufficient count rate from the thin active layers of the samples and an adequate degree of accuracy in determining peak positions.

Single (004) Bragg reflections provided measurement of the lattice constant along the direction of growth $\left(a_{\perp}\right)$ for the strained GaAs layers and strain-inducing $\mathrm{GaAs}_{(1-x)} \mathrm{P}_{x}$ layers. Single (224) Bragg reflections were also acquired for samples \#1 through \#7 from Table 4.1. These reflections provide a measure of $d_{224}$ for the $\mathrm{GaAs}_{(1-x)} \mathrm{P}_{*}$ buffer layers and, in conjunction with $a_{\perp}$, allow the lattice constant within the plane of growth $\left(a_{\|}\right)$to be determined from eq. (4.7).

Figure 5.1 shows typical (004) and (224) scans for a GaAs/GaAs $(1-x) P_{x}$ sample. These reflections were obtajned for sample \#4 from Table 4.1. The (004) Bragg reflection shows clearly resolved peaks for the strained GaAs active layer (left), the GaAs substrate (center), and the $\mathrm{GaAs}_{(1-x)} \mathrm{P}_{x}$ buffer layer (right). The GaAs substrate peak is taken to lie at the known (004) Bragg angle for relaxed GaAs, and the other peak positions are measured relative to this angle. The (224) Bragg reflection shows peaks for the GaAs substrate (left) and the $\mathrm{GaAs}_{(1-x)} \mathrm{P}_{x}$ buffer (right). The strajned GaAs layer is not seen in this scan.

Table 5.1 lists the results for the quantities calculated from the X-ray measurements performed for the eight samples. Once lattice constants have been extracted from the X-ray data, the other quantities shown in Table 5.1 are calculated. The lattice strain along the direction of sample growth $\left(\epsilon_{1}\right)$ is calculated from the definition for sirain and the in-plane strain $\left(\epsilon_{\|}\right)$is related to $\epsilon_{\perp}$ through the tensorial 

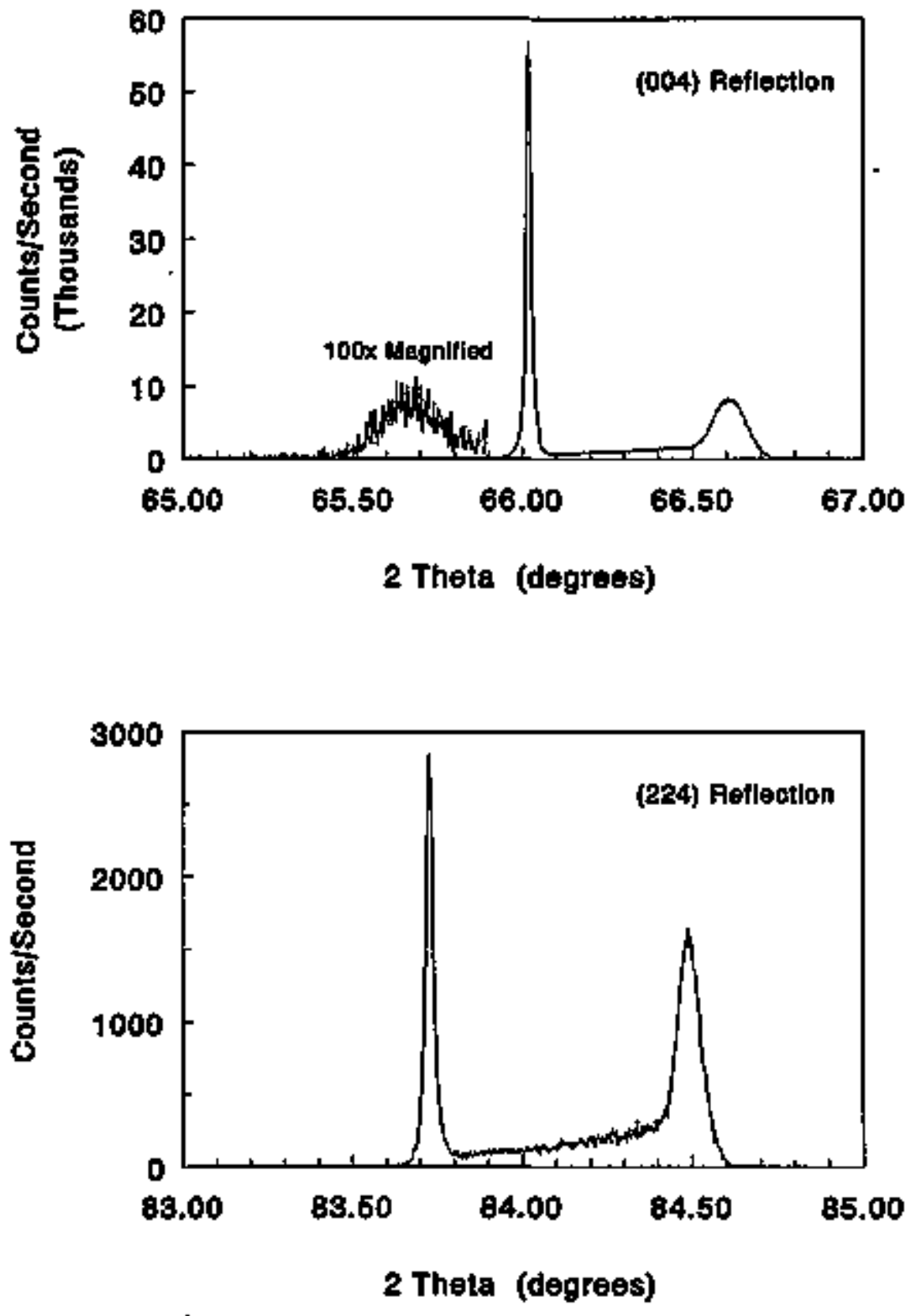

Figure 5.1: (004) and (224) X-ray scans from sample \#4. 


\begin{tabular}{ccccccccc} 
& GaAsP & GaAsP & GaAsP & GaAsP & GaAs & GaAs & GaAs & GaAs \\
Sample & $a_{\perp}(\AA)$ & $a_{\|}(\AA)$ & \% Phosphorus & $\epsilon_{\|}\left(\times 10^{-4}\right)$ & $a_{\perp}(\AA)$ & $\epsilon_{\perp}\left(\times 10^{-3}\right)$ & $\epsilon_{\|}\left(\times 10^{-3}\right)$ & \% Relaxation \\
\hline & & & & & & & & \\
1 & 5.59374 & 5.59680 & 28.8 & 2.87 & 5.69365 & 7.14 & -7.88 & 21.1 \\
2 & 5.58586 & 5.59130 & 32.1 & 5.11 & 5.68910 & 6.33 & -6.99 & 36.3 \\
3 & 5.59490 & 5.60674 & 26.1 & 11.1 & 5.68886 & 6.29 & -6.94 & 15.7 \\
4 & 5.60901 & 5.61700 & 20.0 & 7.46 & 5.68005 & 4.73 & -5.22 & 18.7 \\
5 & 5.59981 & 5.60739 & 24.7 & 7.10 & 5.68512 & 5.63 & -6.24 & 23.5 \\
6 & 5.59966 & 5.61417 & 23.1 & 1.36 & 5.67298 & 3.48 & -3.84 & 44.5 \\
7 & 5.60145 & 5.61144 & 23.3 & 9.35 & 5.66142 & 1.44 & -1.59 & 78.5 \\
8 & $\ldots$ & $\ldots$ & $\ldots$ & $\ldots$ & $\ldots$ & $\ldots$ & $\ldots$ & $\ldots$ \\
9 & $\ldots$ & $\ldots$ & $\ldots$ & $\ldots$ & 5.67243 & 3.38 & -3.74 & $\ldots$ \\
\hline
\end{tabular}

Table 5.1: Resuits of X-ray analysis for the set of photocathode samples. Refer to Table 4.1 for nominal sample parameters. Methods of calculation for the various quantities are described in the text. 
stress-strain relation by[58]

$$
\epsilon_{\|}=\frac{-c_{11}}{2 c_{12}} \epsilon_{\perp}
$$

where $c_{11}$ and $c_{12}$ are elastic stiffness constants for GaAs. The values of $c_{11}$ and $c_{12}$ are well known.[59] This relation is derived by assuming no stresses along the perpendicular direction and equivalent strains in the two jn-plane directions.

The values of the relaxed lattice constant $\left(a_{0}\right)$ and residual in plane strain $\left(a_{\|}\right)$ for the GaAsP buffer layers are calculated simultaneously from the relations

$$
\begin{gathered}
a_{\|}=\left(1+\epsilon_{\|}\right) a_{o} \\
a_{\perp}=\left(1+\epsilon_{\perp}\right) a_{o}=\left(1-\frac{2 c_{12}}{c_{11}} \epsilon_{\|}\right) a_{o} .
\end{gathered}
$$

Strictly speaking, the value of $\left(\frac{2512}{s_{11}}\right)$ is dependent upon the phosphorus fraction of the buffer layer and the equations must be solved iteratively. However, the dependence on phosphorus fraction is not strong (the ratio varies by $2 \%$ between GaAs and GaP) so that a constant value may be used in the equations with negligible effect on the calculated values. Next, the phosphorus content of a buffer layer is calculated from the relaxed lattice constant $\left(a_{0}\right)$ of the layer by linear interpolation between the lattice constants for GaAs and GaP.

The final parameter of interest which may be extracted from the X-ray data is the degree of relaxation within the strained GaAs active layer of a sample. This quantity is is generally expressed as a percentage of the fully strained state. The value which represents the fully strained state for an active layer is taken as the strain which would exist if the active layer possessed an in-plane lattice constant equivalent to that of the underlying GaAsP layer. In this manner, the percent 
relaxation of an active layer is given by

$$
\% R=\left(1-\frac{\epsilon_{\prod a A s}^{G a x}}{\epsilon_{0}^{\text {max }}}\right),
$$

where

$$
\epsilon_{\|}^{m a z}=\frac{a_{\|}^{G a A s P}-a_{o}^{G a A s}}{a_{o}^{G a A s}} .
$$

\subsection{Luminescence Spectra}

A typical luminescencespectrum acquired from the non-thinned region of a strained layer GaAs/GaAs(1-x) $P_{x}$ photocathode is shown in Figure 5.2. The sample, \#1 from Table 4.1, was at room temperature and the pump source was a CW HeNe laser $(633 \mathrm{~nm}, .5 \mathrm{~mW})$. The larger luminescence peak at approximately $710 \mathrm{~nm}$ is due to radiative recombination in the constant phosphorus fraction buffer layer. Recombination radjation from the $100 \mathrm{~nm}$ strajned GaAs layer occurs near 847 nm and is approximately two orders of magnitude weaker than the buffer layer luminescence. By pumping with radiation of energy less than the buffer layer band gap, the luminescence from the buffer is suppressed. However, at such photon energies the GaAs substrate is effectively pumped and produces luminescence over the same wavelength range as the active layer. As described in Chapter 4 , the substrate luminescence is handreds of times more intense than that from the active layer and must be eliminated by removal of the substrate via chemical etching so samples may be pumped near band edge.

All of the strained layer GaAs/GaAs (1 $-x)_{x} P_{x}$ samples were etched, as described in Section 4.3.1, in order to eliminate substrate luminescence. After etching, one 


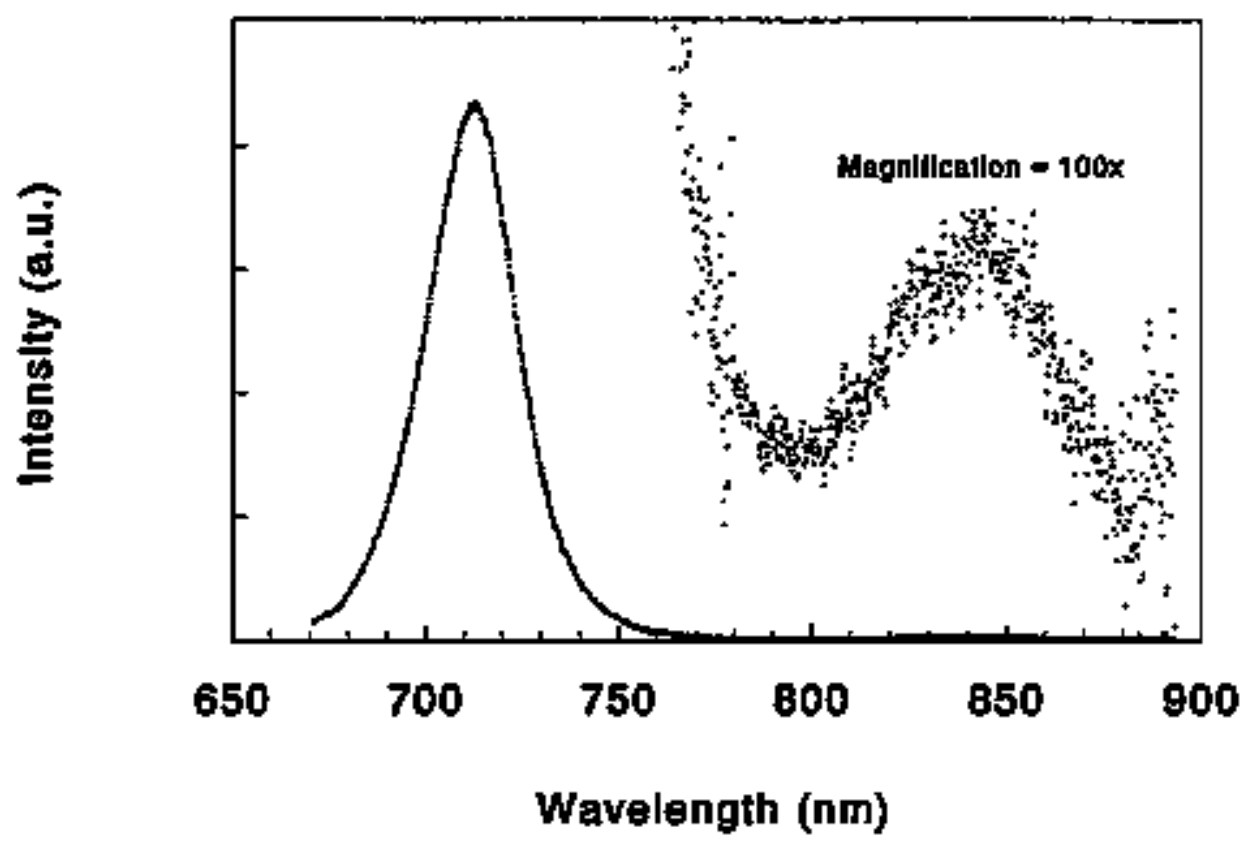

Figure 5.2: Typical room temperature luminescence spectrum from a strained GaAs/GaAs $s_{(1-x)} P_{x}$ photocathode pumped at a wavelength of $633 \mathrm{gm}$. The spectrum is from the non-thinned region of sample \#1. 
sample (\#2 from Table 4.1) showed an interference pattern superimposed upon the active layer luminescence spectrum. The interference pattern appears to be due to a multiple reflection within the thinned region of the sample. The fact that only one thinned sample showed such an interference pattern may be related to the miscut of the substrate. The photocathode sample which exhibited the interference pattern was grown on a (001) GaAs substrate with a $0^{\circ}$ miscut, while the other strained layer samples were grown on substrates with an approximate $2^{\circ}$ miscut. The presence of a miscut may possibly result in a growth surface which is not parallel to the original substrate and, upon etching, the front and back surfaces may not be parallel.

A room temperature luminescence spectrum from this thinned sample is shown in Figure 5.3. The pump source was $750 \mathrm{~nm}$ CW radiation. As seen in Figure 5.3 , interference maxima appear at an interval of approximately $18.5 \mathrm{~nm}$. The joterference fringes can be used to estimate the thickness of the thinned region by

$$
2 n \cdot t=m \lambda_{1}=(m+1) \lambda_{2}=\ldots
$$

An index of refraction of $n=\mathbf{3 . 5 5}$ is calculated from the static dielectric constants for GaAs and GaP [59] as an average for the GaAsP(graded)/GaAsP(constant P)/GaAs structure. Using interference maxima at $\lambda_{1}=850.5 \mathrm{~nm}$ and $\lambda_{2}=832$ $\mathrm{nm}$, the thickness is found to be approximately $t=5.4 \mu \mathrm{m}$ which is in good agreement with the nominal thickness of $5.1 \mu \mathrm{m}$ for the buffer layers and active layer.

The presence of interference fringes inplies that the DAG, which is applied to the substrate-side of the thinned region in order to prevent reflections, is not perfectly suppressing reflections. One can estimate the minimum refectivity re 


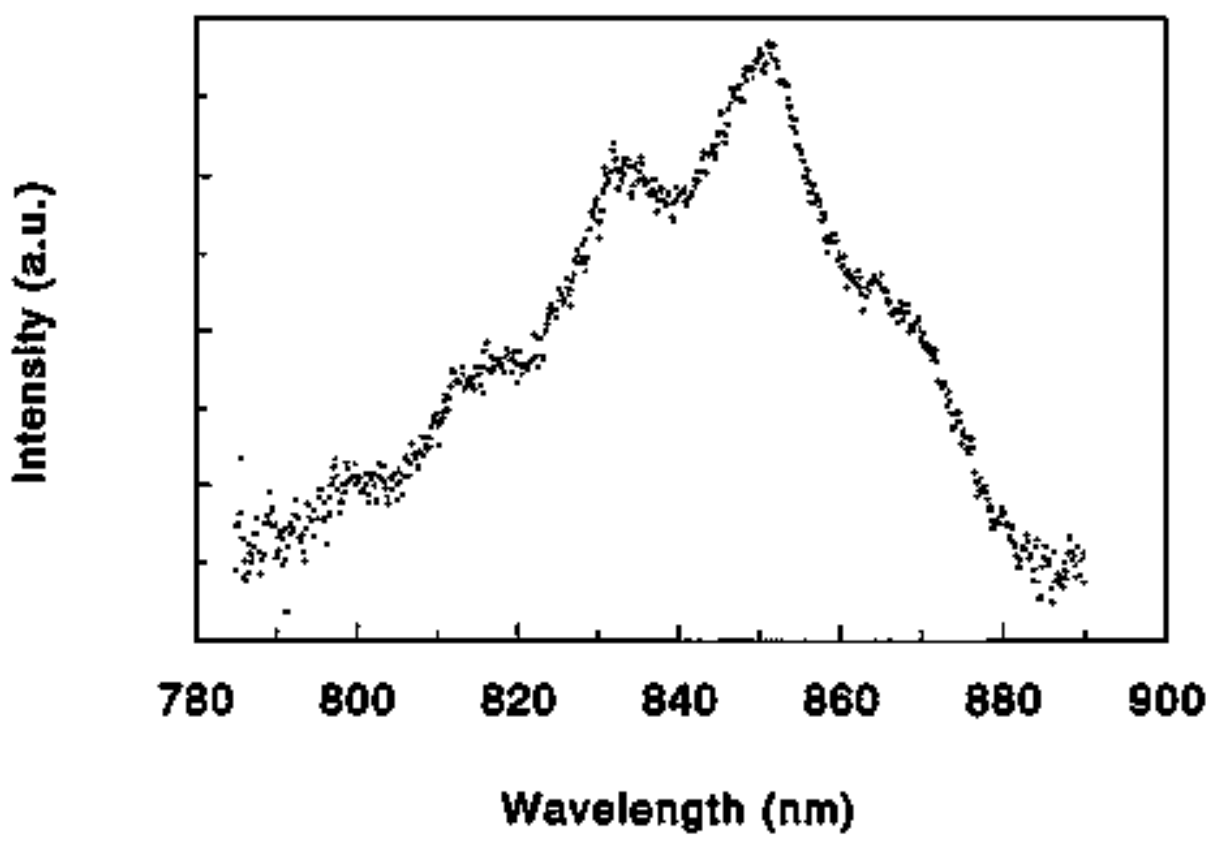

Figure 5.3: Room temperature luminescence spectrum from active layer of sample \#2 (etched region) showing interference fringes. Pump wavelength $=750 \mathrm{~nm}$. 
quired at the GaAs/DAG interface to produce interference fringes of the observed magnitude. Doing so, the minimum necessary reflectivity is found to be as small as $1 \%$, which is an acceptable level.

Although substrate etching was necessary so that luminescence could be observed while pumping samples near band edge, there is some information which may be obtained from the luminescence spectra of non-thinned strained layer photocathodes. The luminescence spectrum shown in Figure 5.2 was obtained from a region of the sample which was not thinned by etching. It is comprised of two spectra obtained at different spectrometer grating positions with each spectrum requiring an acquisition time of only five seconds. Such short acquisition times are made possible by the use of the intensified diode aray detector, described in Chapter 4, and are an attractive aspect of luminescence-based characterization techniques. Both the $\operatorname{GaAs}_{(1-x)} P_{x}$ layer and strajned GaAs layer luminescence peales are used to characterize the photocathode samples.

\subsubsection{Determination of Phosphorus Content in $\mathrm{GaAs}_{(1-x)} \mathbf{P}_{\boldsymbol{x}}$}

The physical and electrical properties of a II-V ternary alloy may be described functionally as a combination of the two III-V binary constituents. Often, the properties follow a simple lizear relationship with the alloy mixture as described by Vergad's Law. The dependence on phosphorus fraction of the GaAs(1-x) $P_{r}$ band gap has, however, been found to be slightly quadratic.[60]

$$
E_{g}(x)=\left(1.424+1.15 \cdot x+0.176 \cdot x^{2}\right) \mathrm{eV}
$$

Here, $E_{g}(x)$ is the band gap energy for intrinsic $\operatorname{GaAs}_{(1-x)} \mathrm{P}_{x}$ at room temperature. 
The luminescence spectrum for a direct band gap semiconductor is directly related to the band gap energy of the semiconductor. Doping level and temperature are factors which affect the explicit relationship between band gap and the luminescence peak maximum. However, by assuming a situation where doping level and temperature are constants, one expects the lnminescence peak energy to depend on phosphorus content in the same manner as the band gap. The luminescence peak position for $\mathrm{GaAs}_{(1-\infty)} \mathrm{P}_{x}$ is, therefore, expected to follow

$$
E_{P L}(x)=E_{P L}(x=0)+\left(1.15 \cdot x+0.176 \cdot x^{2}\right) \mathrm{eV}
$$

where $E_{P L}(x)$ is the observed laminescence peak energy from a $G a A s_{(1-x)} P_{x}$ layer.

Room temperature Juminescence spectra from the $\operatorname{GaAs}_{(1-x)} \mathrm{P}_{z}$ layer of the seven $\mathrm{GaAs}_{3} / \mathrm{GaAs}_{(1-x)} \mathrm{P}_{x}$ photocathode structures were recorded in order to verify the relationship between peak position and phosphorus content. All spectra were obtained while pumping with a CW HeNe laser ( $633 \mathrm{~nm}, .5 \mathrm{~mW})$ and corrected for spectrometer/diode array response as described in Section 4.9.3. Peak positions for the spectra were determined by 'eye' and are estimated to be accurate to within $2 \mathrm{meV}$ (approximately $1 \mathrm{~nm}$ ). This data is shown in Figure 5.4 plotted agajnst the phosphorus content of the layers as determined by X-ray diffraction. The solid line in Figure 5.4 represents a least squares fit of the relationship given by eq. (5.8) where the only free parameter is the GaAs $(x=0)$ peak position. The good agreement between the fit and the data indicates that the phosphorus content of the buffer layer may be determined accurately using the observed luminescence peak position and the relationship given by Casey and Panish (eq. (5.8)).

Determination of the phosphorus content of the $G a A s_{(1-x)} P_{x}$ buffer layer by 


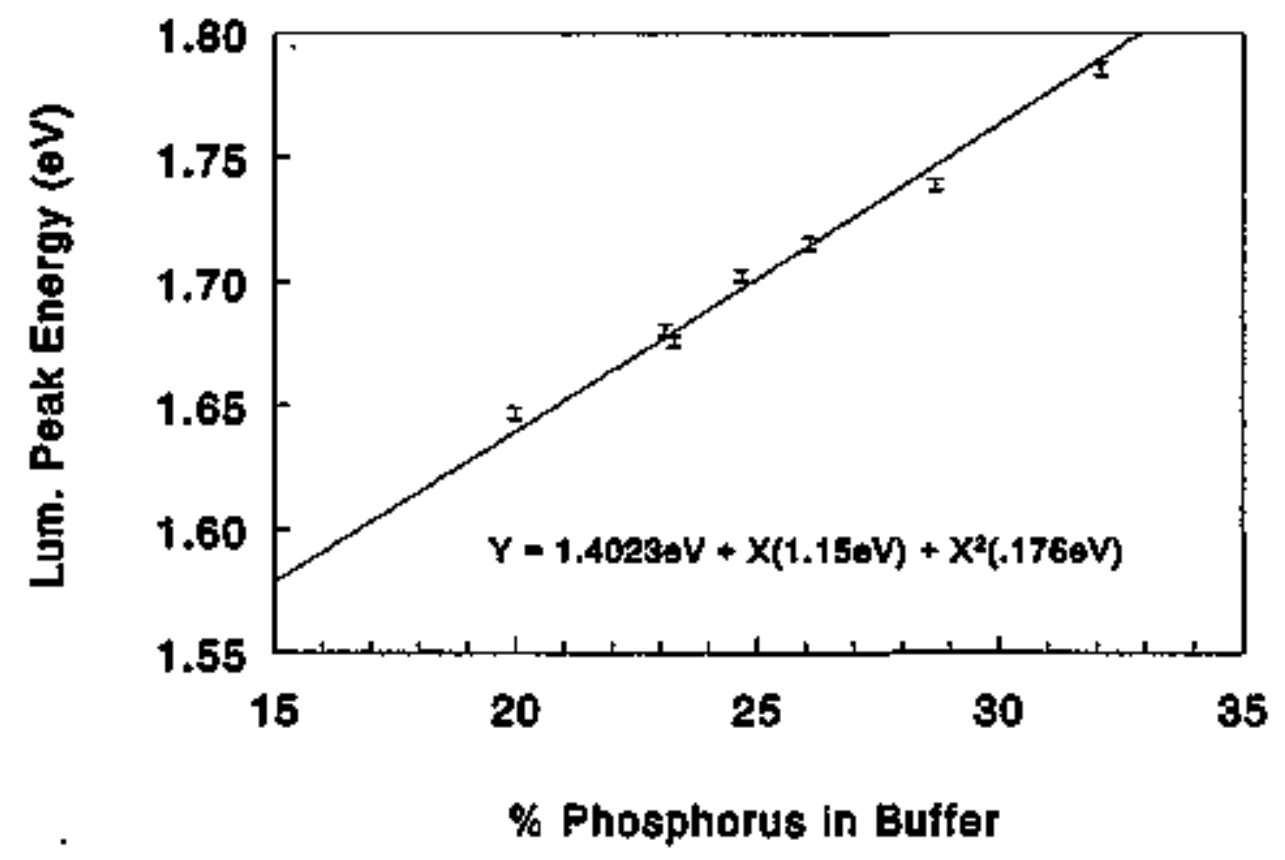

Figure 5.4: Room temperature GaAsP buffer layer luminescence peak energy plotted against the phosphorus content as measured by $\mathrm{X}$-ray diffraction. Soljd line is a least squares fit of eq. (5.8) with $E_{P L}(x=0)$ as a free parameter. 
luminescence techniques is extremely useful for wafer uniformity mapping. The luminescence measurements are non-destructive and peals positions may be recorded in real time when using the intensified diode array detector. Figure 5.5 shows two examples of phosphorus content mapping across a 2 in. photocathode wafer. Wafer $\mathbf{A}$ is that from which sample \#3 was cut. The two circular areas seen are places where circular cathodes were cut from the wafer before mapping. The + symbols represent positions where a buffer layer luminescence spectrum was recozded, and the solid lines represent the approximate constant-phosphorus surfaces. Wafer B is shown as a comparison of phosphorus content uniformity. This MOCVD grown photocathode waier was obtained from another vendor. It is clearly much less uniform than wafer A, exhibiting a variation in phosphorus content of over $8 \%$ (absolute) across its full surface. Such non-uniformity is undesirable because it affects the ability to predict photocathode material performance based on testing of a single sample cut from the material.

\subsubsection{Determination of Epilayer Strain}

The room temperature luminescence spectra obtained from the strained layer photocathode structures may be used to estimate the degree of biaxial compressive strain within a given $\mathrm{GaAs}$ active layer. The band gap of $\mathrm{GaAs}$ is affected by the biaxial compression associated with pseudomorphic growth on $\mathrm{GaAs}_{(1-x)} P_{x}$. With the presence of strain, the band gap energy shifts in a well defined manner and is directly observed as an equivalent shift of the luminescence peak. An example of the magnitude of strain-jnduced luminescence peak shift is shown in Figure 5.6. 


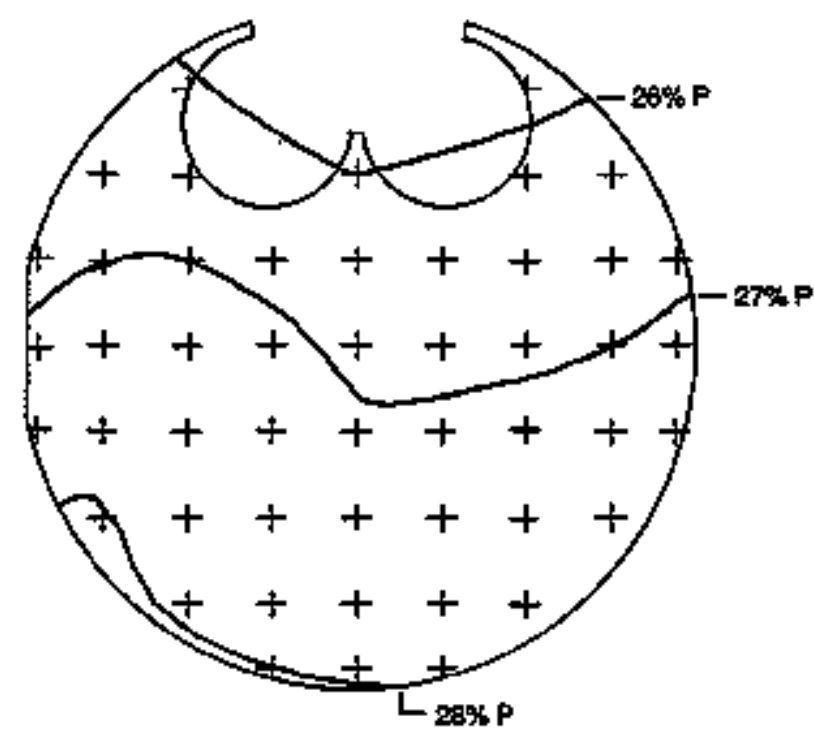

A

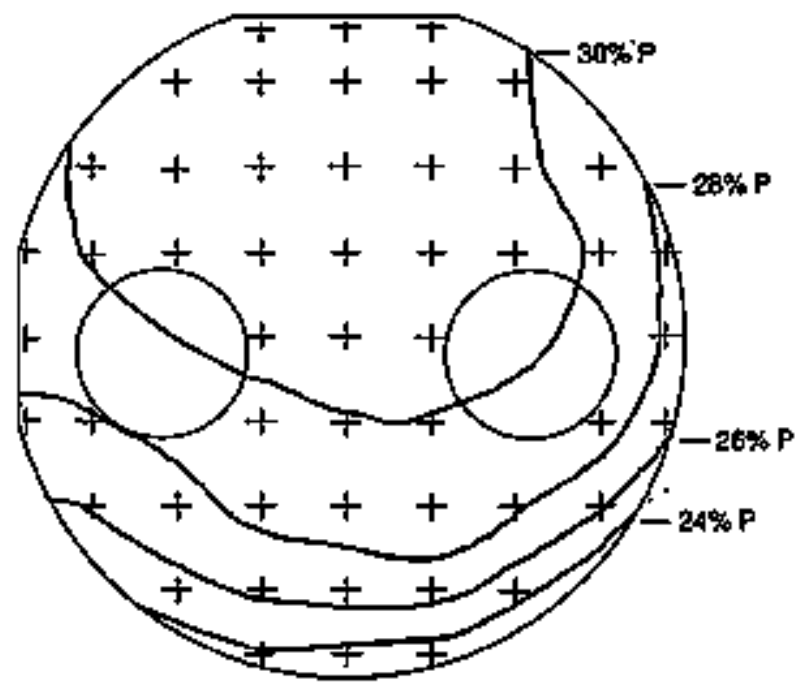

B $\quad$ indloges

Figure 5.5: Examples of wafer mapping using luminescence measurements. Here, the phosphorus content of the $\operatorname{GaAs}_{[([-x]} \mathrm{P}_{x}$ layer is mapped. 


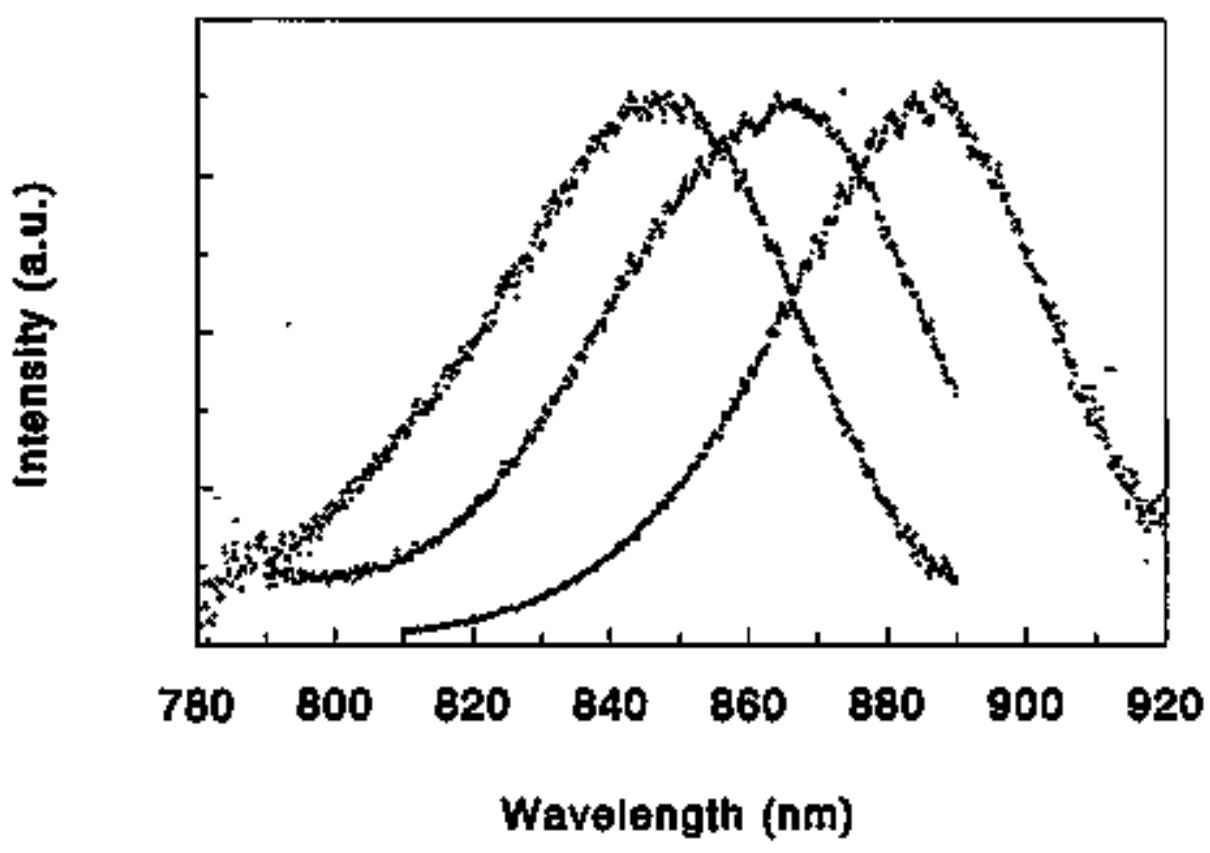

Figure 5.6: Room temperature luminescence spectra from the active layers of (left to right) sarnples \#1, \#6 and \#8. Purnp wavelength $=750 \mathrm{~nm}$.

This figure shows three room temperature luminescence spectra from samples of varying strains scaled to approximately equal intensity. The spectra were recorded while pumping with $750 \mathrm{~nm} \mathrm{CW}$ radiation from the Tj:sapphire laser. The three spectra, as viewed from left to right, correspond to measurements taken from the thinned region of samples \#1, \#6 and \#8, respectively. Each spectrum represents the sum of four separate acquisitions which correspond to the four possible pump bearn/luminescence helicity combinations of $\sigma^{+}$(pump) $/ \sigma^{-}(\mathrm{lum}), \sigma^{+} / \sigma^{+}$, $\sigma^{-} / \sigma^{+}$, and $\sigma^{-} / \sigma^{*}$. The sum represents a luminescence spectra acquired with no polarization analysis while pumping with a non-polarized source.

Active layer luminescence spectra were recorded for all of the GaAs $/ \mathrm{GaAs}_{\{1-x\}} P_{x}$ photocathode samples. A luminescence spectrum from the actjve layer of the 
$\mathrm{GaAs} / \mathrm{AJ}_{30} \mathrm{Ga}_{70} \mathrm{As}$ sample was also acquired in order to represent the situation of zero residual strain. These spectra were all empirically fit with curves of the form

$$
I(\lambda)=C_{0} \exp \left(-0.5\left(\ln \left(\left(C_{1}-\lambda\right) / C_{2}\right) / C_{3}\right)^{2}\right)
$$

where $C_{\mathrm{D}}-C_{3}$ are parameters of the fit. This curve is known as Log-Normal and was used because it provided a good fit to the luminescence line-shape. The spectrum from sample \#2 (Figure 5.3) was not used because of the difficulty in determining the peak center in the presence of interference fringes. The luminescence peak positions (measured in $\mathrm{eV}$ ) were then plotted against the residual strains in the active layers of the samples as measured by X-ray diffraction. The plot is shown in Figure 5.7 with a linear least squares fit to the data.

The radiative transitions comprising the luminescence spectra are expected to be predominantly (bat not exclusively) to $\left.\mid \frac{3}{2}, \frac{3}{2}\right)$ states. Recombination transitions to this band are favored by three to one over transitions to the $\left(\frac{3}{2}, \frac{1}{2}\right)$ valence band from selection rules alone, and Fermi statistics further favor transitions to the higher lying (in energy) $\left(\frac{3}{2}, \frac{3}{2}\right)$ valence band. It is therefore appropriate to compare the observed strain dependence of luminescence peak position with the expected strain dependence of the conduction band to $\left|\frac{3}{2}, \frac{9}{2}\right\rangle$ valence band transition. The strain dependence of this transition is given by $\Delta E=\delta_{h}+\delta_{s} / 2$, where $\delta_{h}$ and $\delta_{s}$ are defined in eqs. (2.27) and (2.28). Using deformation potential values of $a=-9.77$ $\mathrm{eV}$ and $b=-1.7 \mathrm{eV}[25]$ and elastic stiffuess constants of $c_{11}=11.88 \times 10^{11} \mathrm{dyn} / \mathrm{cm}^{2}$ and $c_{12}=5.38 \times 10^{11} \mathrm{dyn} / \mathrm{cm}^{2}[59]$, one predicts the strain dependence to be $\Delta E$ $=(-7.45 \mathrm{eV}) \epsilon_{\|}$, which is in reasonable agreement with the observed dependence.

Luminescence peaks from the GaAs active layers of the photocathode structures 


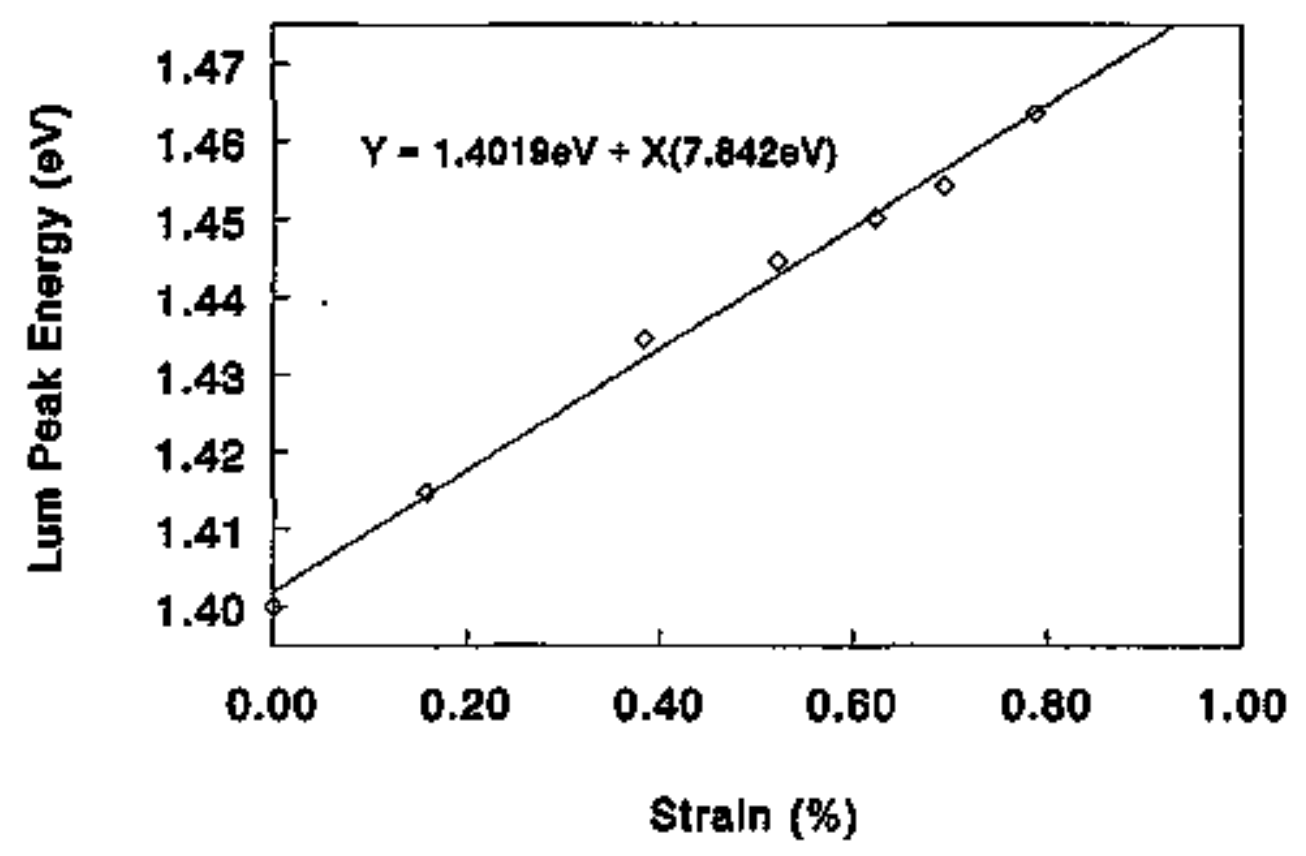

Figure 5.7: GaAs active layer luminescence peak energy vs. in-plane strain $\left(\epsilon_{\|}\right)$ as measured by $\mathrm{X}$-ray diffraction (strain values are actually negative). Pump wavelength $=750 \mathrm{~nm}$. Solid line is a linear least squares fit to data. 
may be observed without the special preparation procedure of substrate removal by etching (see Figure 5.2). However, in order to observe the active layer luminescence from a sample which has not been thinned, one must use a pump beam of short enough wavelength such that it will be absorbed within the GaAs(1-2) $\mathrm{P}_{-}$layer, thus avoiding optical pumping of the sabstrate. As seen in Figure 5.2, under these purnp conditions the active layer luminescence peak may lie on the non-flat tail of the $\mathrm{GaAs}_{(1-x)} \mathrm{P}_{x}$ layer luminescence, thereby complicating accurate determination of the peak position. Nevertheless, these luminescence spectra still provide a fairly accurate and reliable measurement of the residual strain within the active layer of a photocathode sample, and such measurements are routinely performed.

GaAs active layer luminescence measurements are also useful in predicting the performance of photocathode materials with respect to maximum electron spin polarization. Figure 5.8[61] shows the maximum observed photo-mitted electron spin polarization plotted against the strain-induced luminescence peak shift for several photocathode samples. All data points are representative of the Spire[31] GaAs/GaAss $s_{(1-x)} P_{2}$ structure. The luminescence spectra were acquired at room tenperature with a $\mathrm{HeNe}(633 \mathrm{~nm})$ punp source, and the shift was measured relative to an expected zero-strain peak energy obtained from a plot similar to Figure 5.7. The luminescence measurement is well correlated with the obtainable electron spin polarization. 


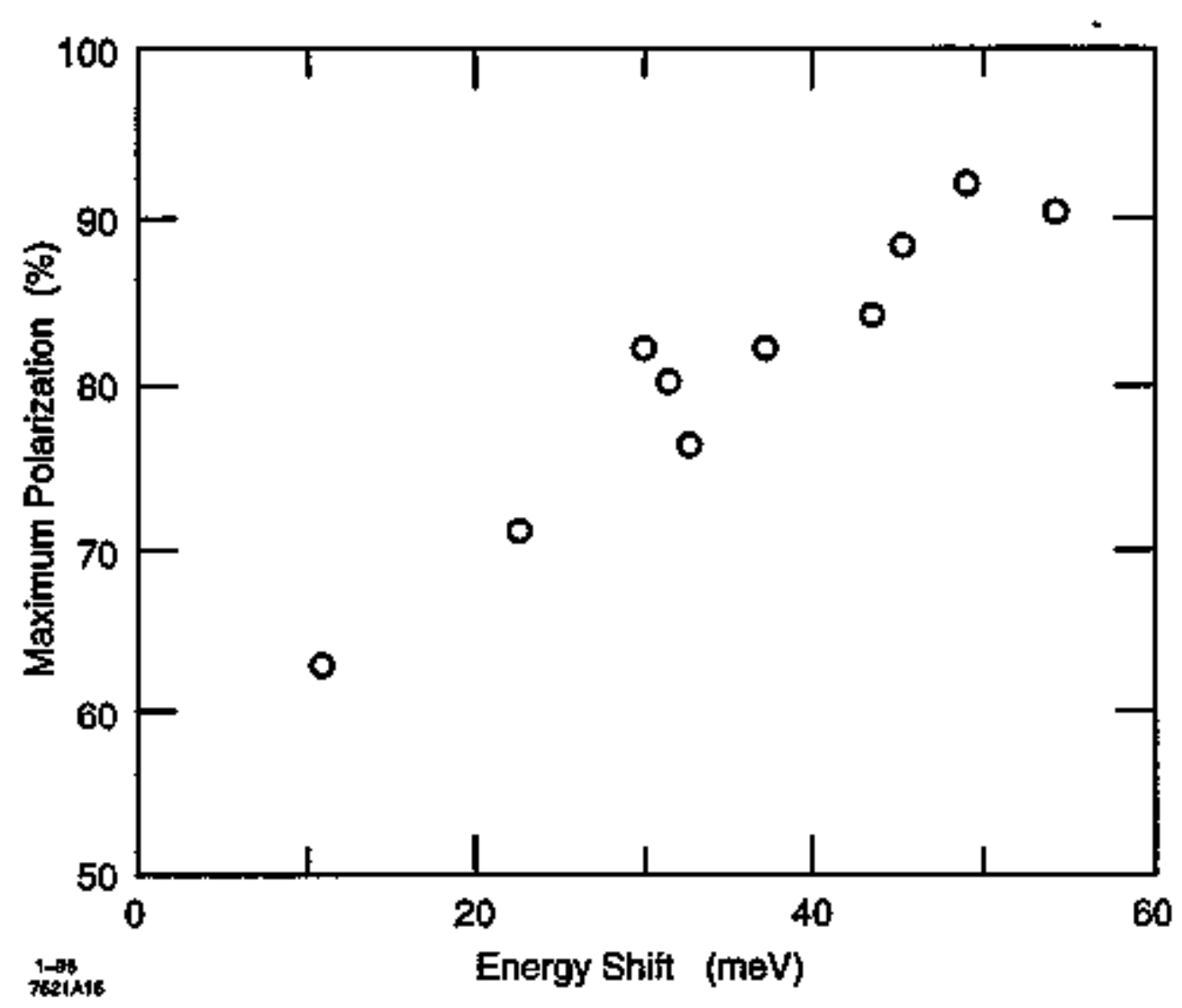

Figure 5.8: Maximum photo-emitted electron spin polarization plotted against the observed luminescence energy shift for several samples of strained GaAs on $\operatorname{GaAs}_{(1-x)} \mathrm{P}_{\boldsymbol{x}}$. 


\subsubsection{Line-Shape of Luminescence Spectra}

The general line-sbapes of the luminescence spectra shown in Figure 5.2, and more clearly in Figure 5.6, indicate significant deviation from the line-shape expected from the assumption of parabolic energy bands. The spectra exhibit low energy tails which are explained by exponentjal or gaussian tails on the valence and conduction band density of states $(\rho(E))$ which extend into the forbidden gap near $\vec{k}$ $=0 .[62][63]$ These so called band tails are described by several models[64][65] as an effective smearing of the density of states due primarily to spatial fluctuations in the ionized impurity density. Consequently, within these models, the degree of band tailing is related to the doping level. The Kane model[65], for example, gives the density of states as

$$
\rho(E)=\left(\frac{m^{* 3 / 2}(2 \eta)^{1 / 2} V}{\pi^{2} \hbar^{3}}\right) \times \frac{I}{\pi^{1 / 2}} \int_{-\infty}^{x}(x-\zeta)^{1 / 2} \exp \left(-\zeta^{2}\right) d \zeta,
$$

where

$$
\eta=\left(e^{2} / 4 \pi \epsilon_{d}\right)\left[2 \pi\left(N_{D}^{+}+N_{A}^{-}\right) L\right]^{1 / 2} .
$$

Here, $m^{*}$ is the effective mass, $V$ is the unit cell volume, $e$ is the electron charge, and $L$ is the screening length. The density of states given by eq. (5.10) has the form $\rho \sim \exp \left(-E^{2} / \eta^{2}\right)$ for energy less than band edge $(E<0)$ and approaches the parabolic band form of $\rho \sim E^{1 / 2}$ for large energy. The dependence of the band tails on doping level is carried in $\eta$. In addition, the tails may exhibit a temperature dependence because the screening length will, in general, depend on temperature. For instance, the Debye (free carrier) screening length is given as $L=\left(\epsilon_{\mathrm{d}} k_{B} T / p e^{2}\right)^{1 / 2}$ ( $p$ is the hole density) which results in a $T^{3 / 4}$ dependence for $\eta \cdot$ 
The presence of band tails will affect the absorption edge of each of the $J=$ $3 / 2$ valence bands, so that the transition from optical pumping of both bands to selective pumping of the $J=3 / 2, \dot{m}_{j}=3 / 2$ valence band will be smeared in photon energy. In fact, the presence of tails may actually limit the electron spin polarization which may be obtained. Band tailing will therefore be considered when analyzing luminescence circular polarization data.

\subsection{Circular Polarization of Active Layer Luminescence}

As discussed in Chapter 3, the circular polarization of luminescence from an epitaxial layer of GaAs is related to the degree of spin polarization of the photo-exited conduction electrons and to the recombination radiation coupling factor. The expression for $P_{\gamma}$ was given as $P_{\gamma}=-P_{e}-\left(h \nu_{i n}\right) P_{h}\left(h \nu_{p i}\right) R$, where $R$ is assumed constant. From this expression, it is seen that the pump photon energy (wavelength) dependence and luminescence photon energy (wavelengtb) dependence of luminescence circular polarization are carried exclusively by $P_{c-}$ and $P_{h}$ respectively, allowing for separate measurements of both $P_{0}-$ and $P_{h}$.

\subsubsection{Dependence on Luminescence Wavelength}

Roon temperature luminescence spectra from the nine photocathode samples were recorded for the purpose of measuring the wavelength dependence of the luminescence circulat polarization. The spectra were obtained while pumping with the $\mathrm{CW}$ Ti:sapphire laser tuned to $750 \mathrm{~nm}$. It was necessary to pump one of the samples with $780 \mathrm{~nm}$ radiation in order to achieve the necessary suppression of $\mathrm{GaAs}_{1-x} \mathrm{P}_{x}$ 
layer optical pumping relative to the strained GaAs layer. The pump power for all samples was approximately $3 \mathrm{~mW}$. In order to measure luminescence circular polarization asymmetries, all four pump helicity/luminescence helicity combinations were recorded while the exposure and pump power were held constant. The circular polarization was then calculated as

$$
P_{\gamma}(\lambda)=-1 \cdot\left(\frac{I^{++}(\lambda)+I^{--}(\lambda)-I^{+-}(\lambda)-I^{-+}(\lambda)}{I^{++}(\lambda)+I^{--}(\lambda)+I^{+-}(\lambda)+I^{-+}(\lambda)}\right) .
$$

Here, $I(\lambda)$ is the intensity (ot total counts) of the given spectrum at the wavelength $\lambda$, and the superscript notations $(++,-\infty,+-,-+)$ denote the four possible pump/luminescence belicity combinations. The sign of the polarization is made positive for ease of plotting by multiplication with -1 as indicated in eq. (5.12). The resulting polarization curves for the samples are shown in Figures 5.9 through 5.17 with the corresponding luminescence spectra. The spectra shown here represent the sum over all four helicity combinations as described previously.

All of the circular polarization curves from strained layer photocathodes presented in Figures 5.9-5.17 show the expected behavior of polarization enhancement with increasing wavelength (decreasing photon energy) as discussed in Chapter 3, while the non-strained sample (\#8) does not show any enhancement. When compared with the X-ray measured strains of each sample, the family of circular polarization curves also reveals that the onset of polarization enbancement moves towards sborter wavelengths (larger photon energies) with increasing strain as expected from the strain-induced hydrostatic shift of the valence band edge. The data from sample \#9 does not follow this trend, though. This sample has a luminescence peak as well as an enhanced polarization at shorter wavelengths than 


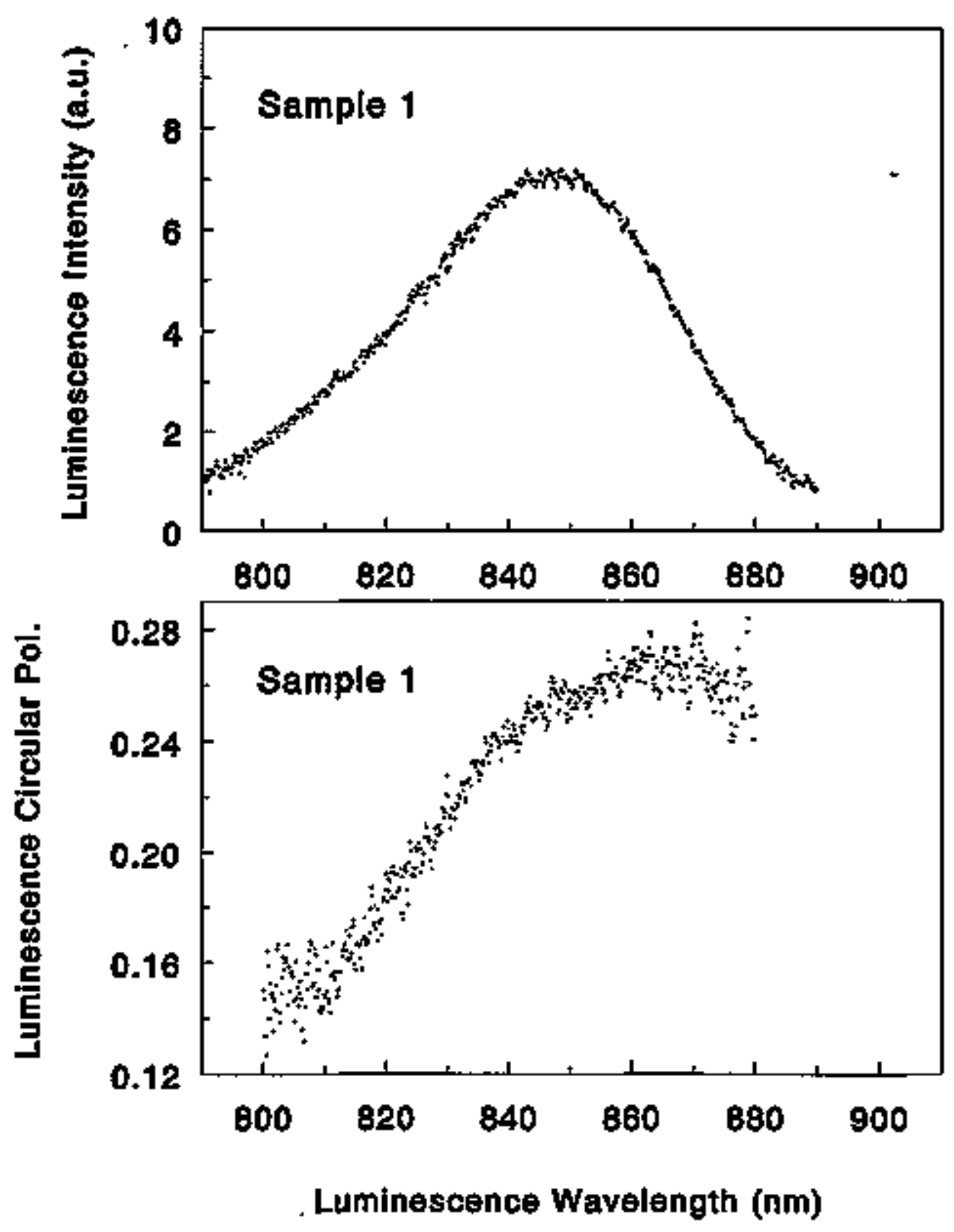

Figare 5.9: Room temperature luminescence spectrum and corresponding circular polarization of laminescence for sample \#1. Pump wavelength $=750 \mathrm{~nm}$. 


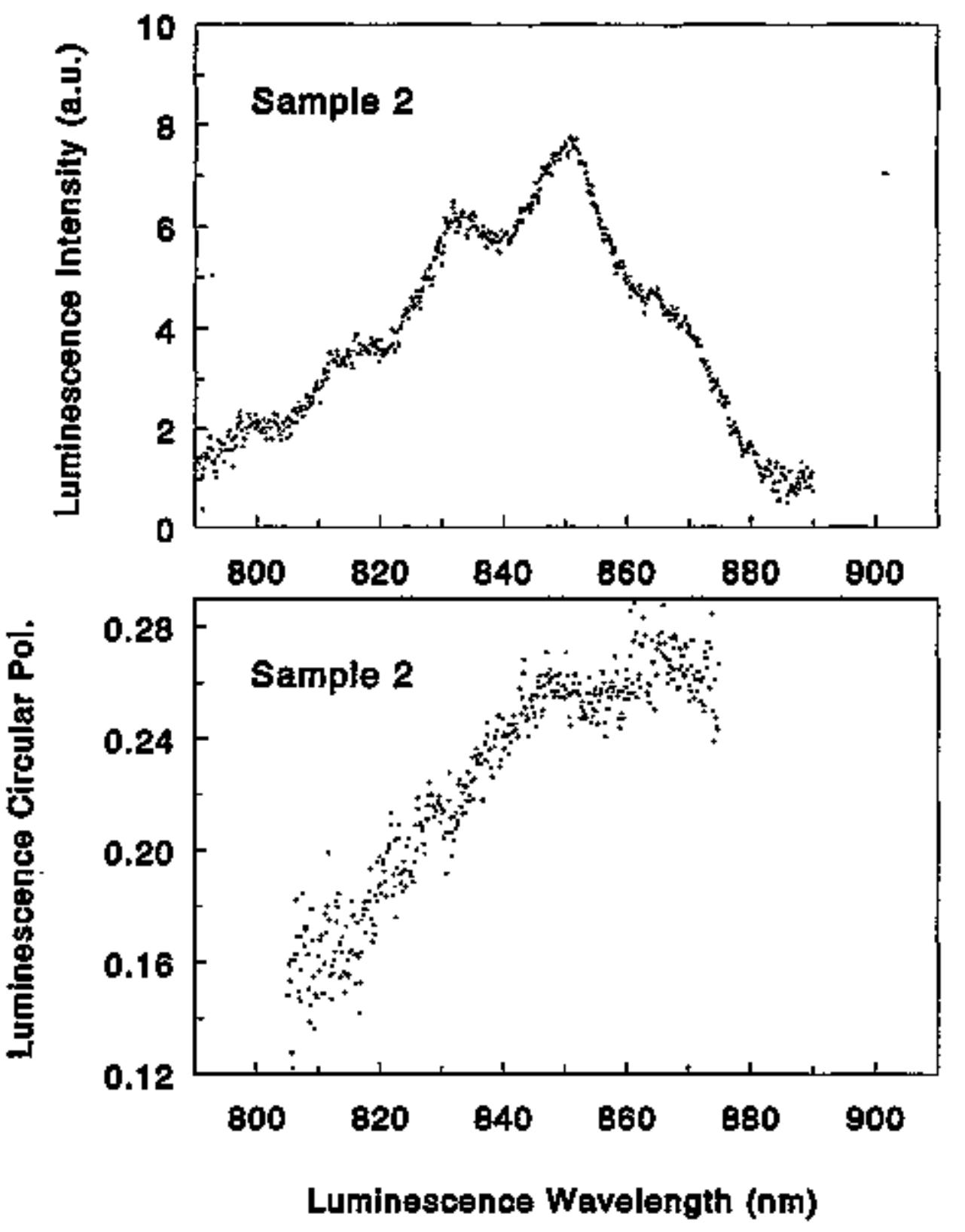

Figure 5.10: Room temperature luminescence spectrum and corresponding circular polarization of luminescence for sample \#2. Punnp wavelength $=750 \mathrm{~nm}$. 


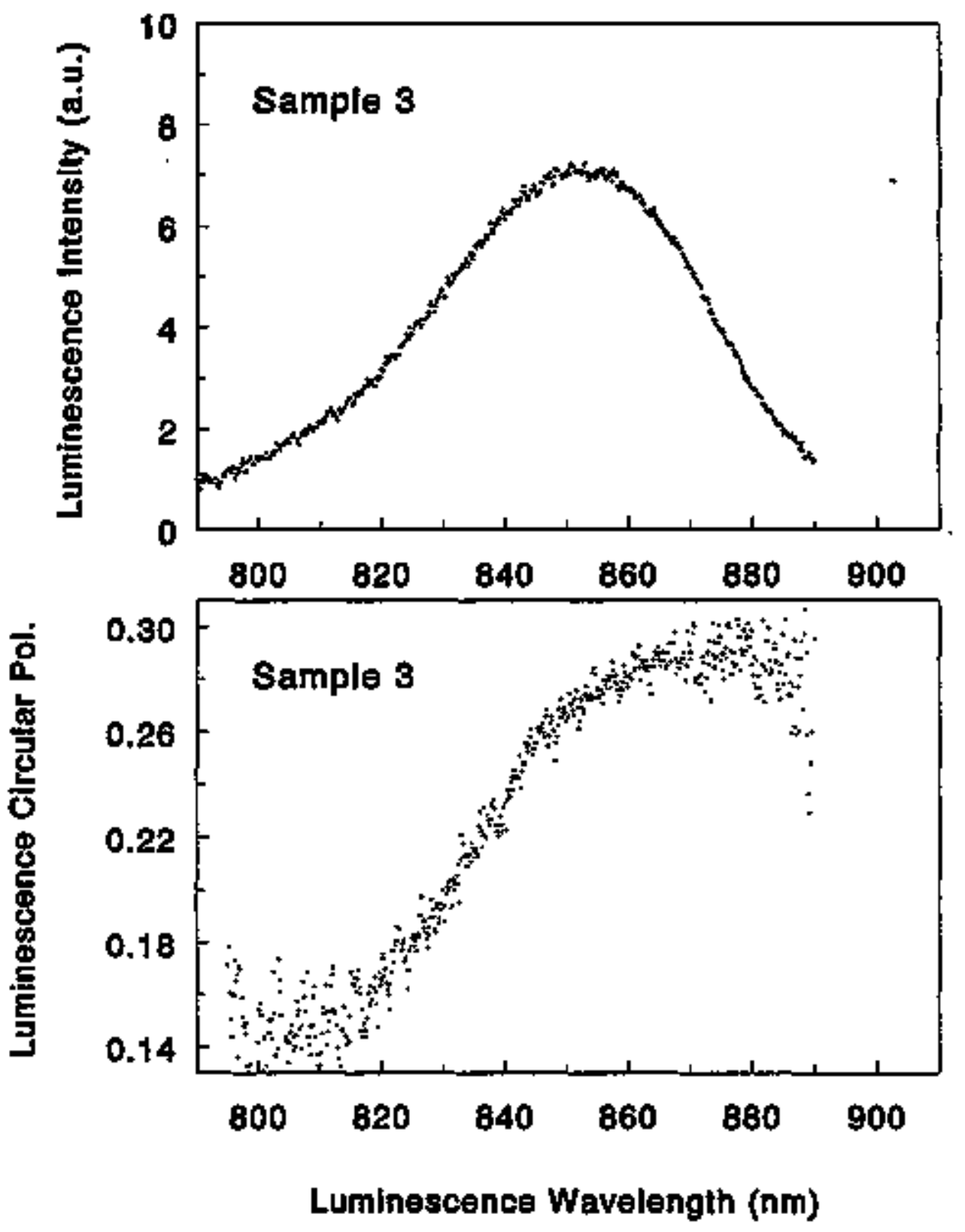

Figure 5.11: Room temperature luminescence spectrum and corresponding circular polarization of luminescence for sample \#3. Pump wavelength $=750 \mathrm{~nm}$. 


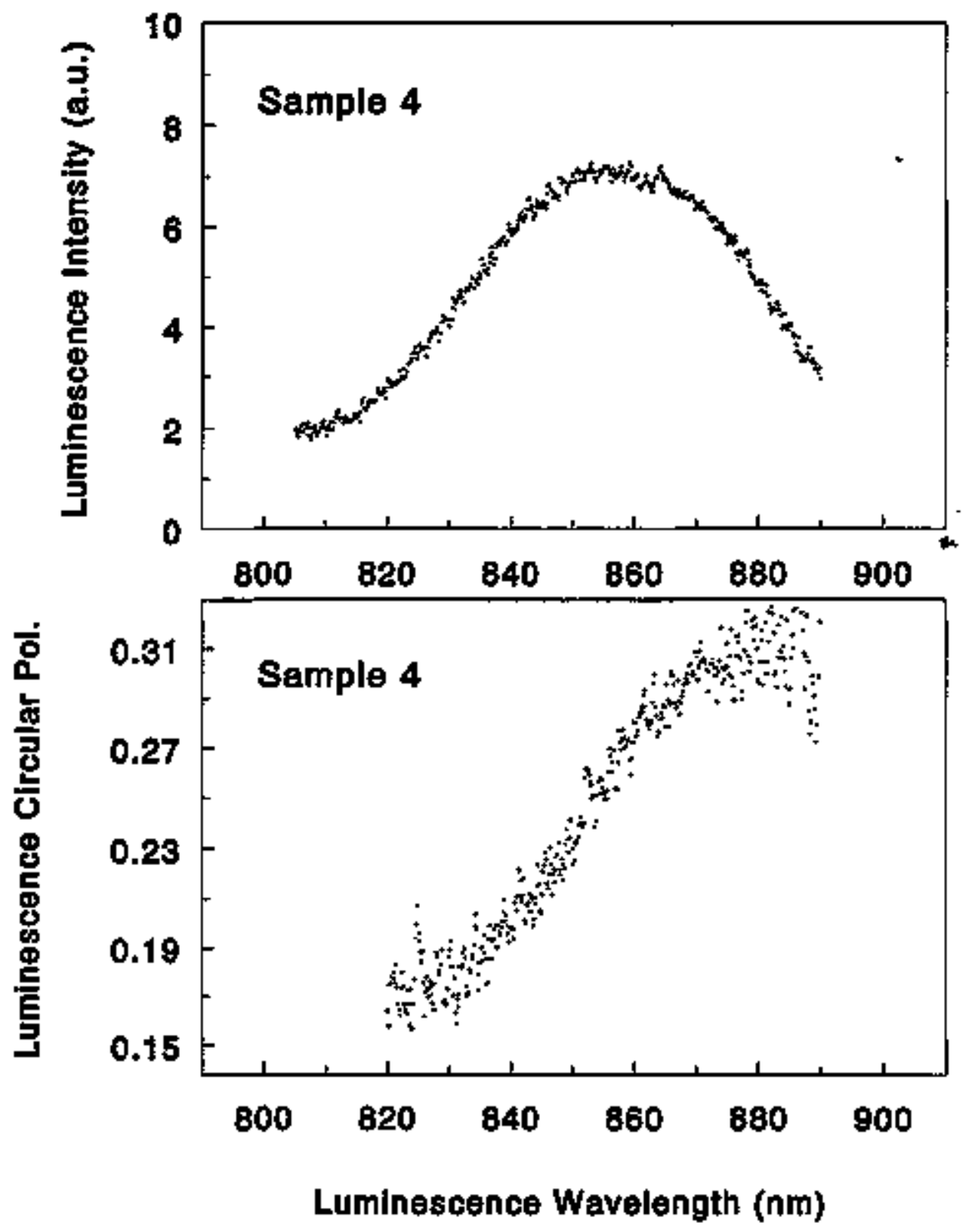

Figure 5.12: Room temperature luminescence spectrum and corresponding circular polarization of luminescence for sample \#4. Pump wavelength $=780 \mathrm{~nm}$. 


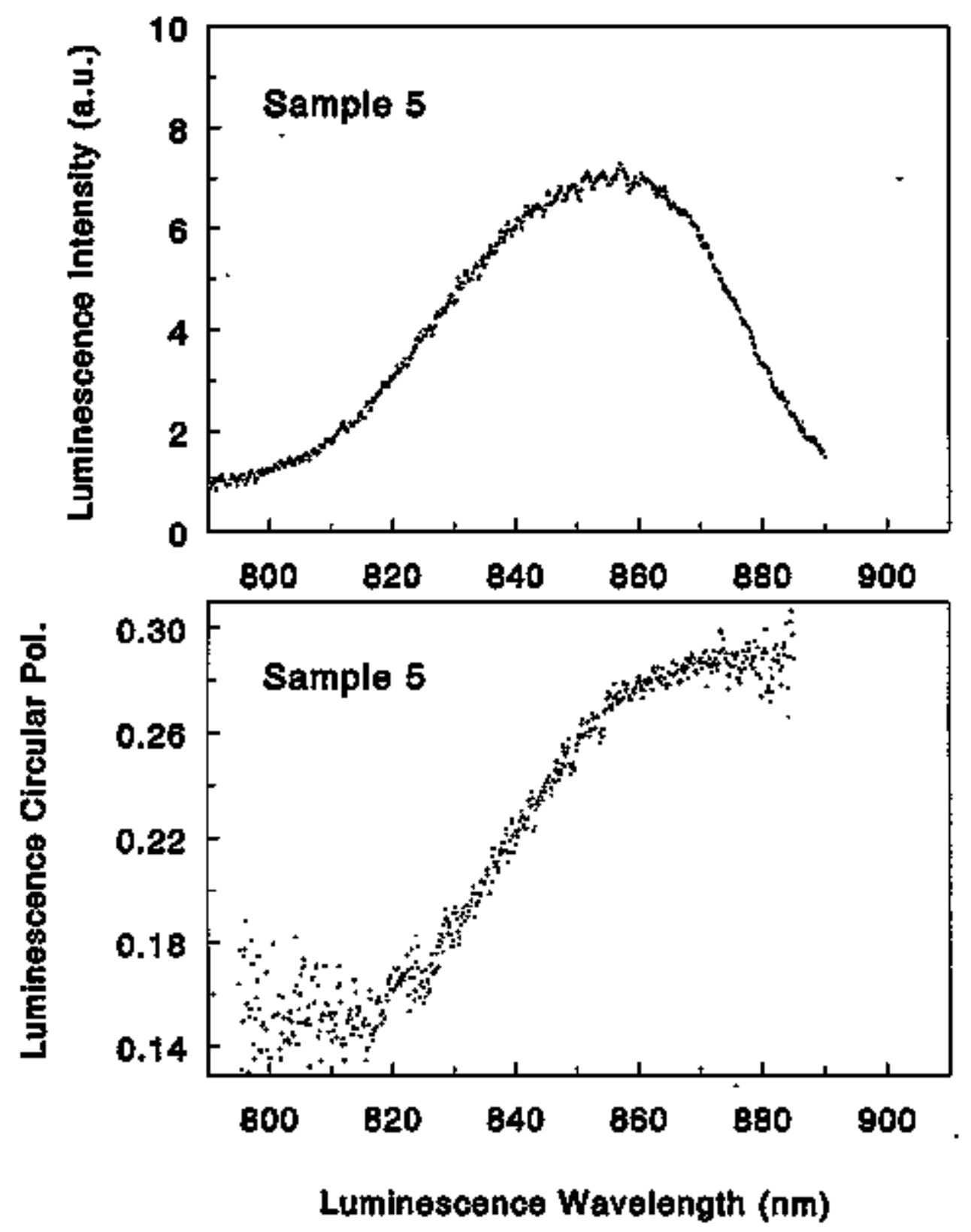

Figure 5.13: Room temperature luminescence spectrum and corresponding circular polarization of luminescence for sample \#5. Pump wavelength $=750 \mathrm{~nm}$. 


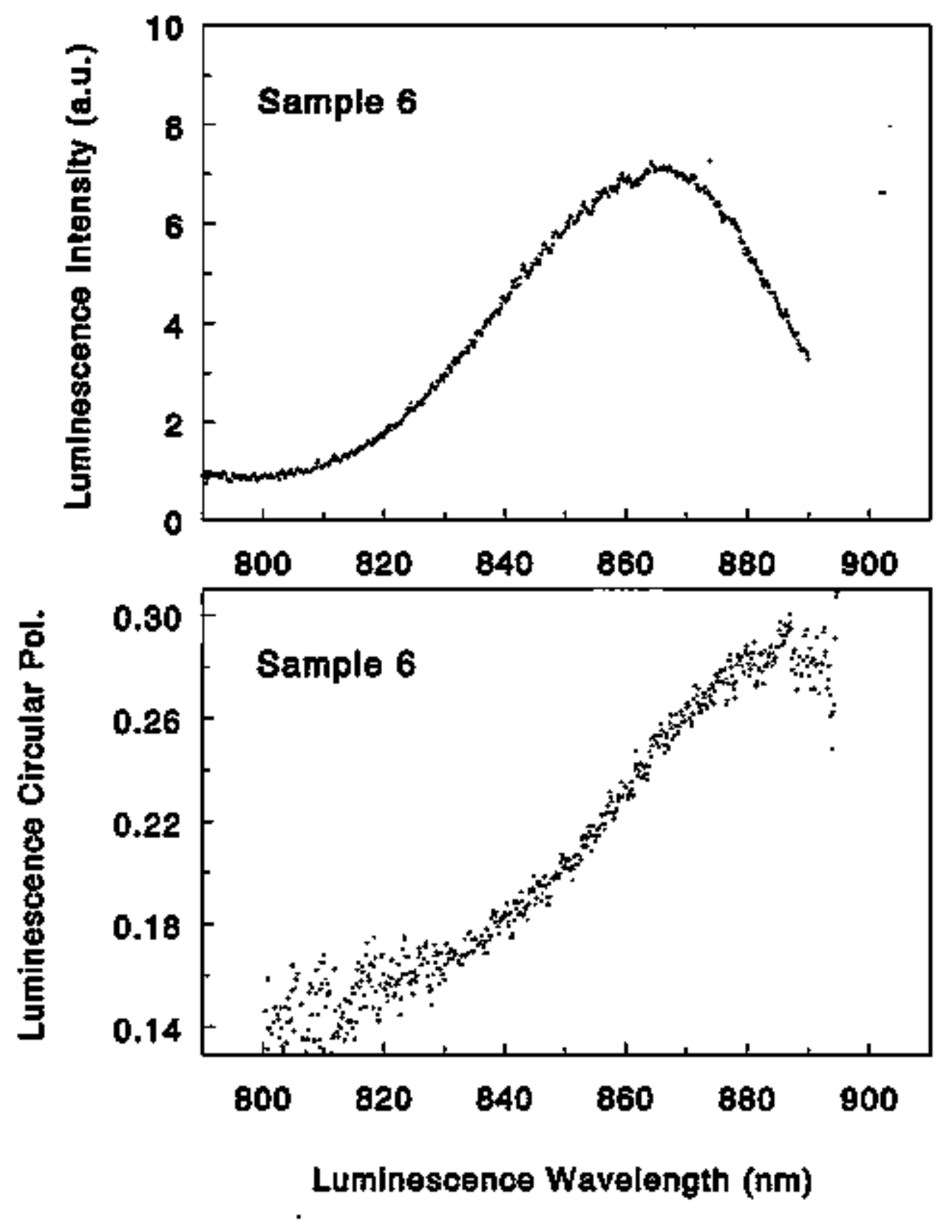

Figure 5.14: Room temperature luminescence spectrum and corresponding circular polarization of luminescence for sample \#6. Pump wavelength $=750 \mathrm{~nm}$. 


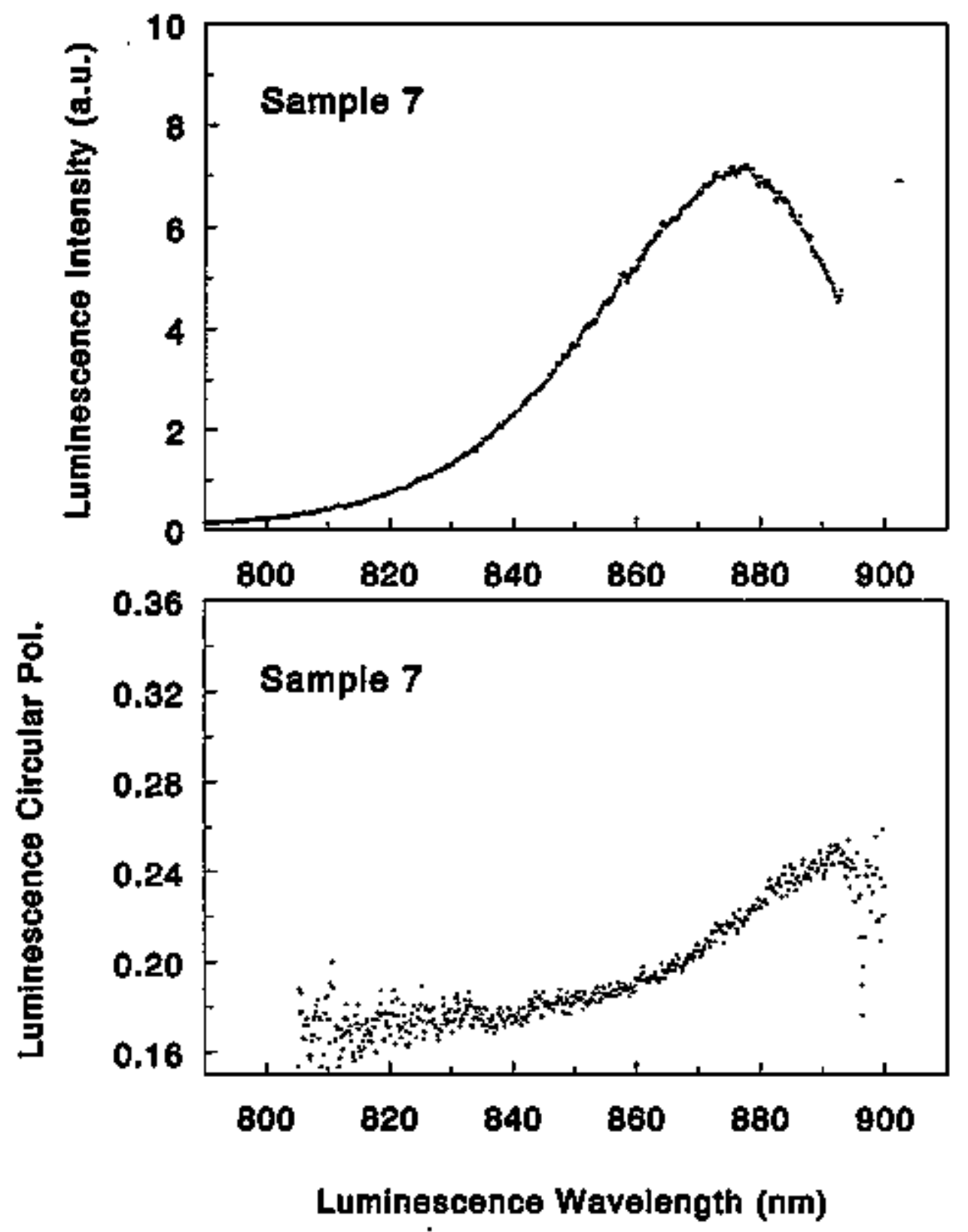

Figure 5.15: Room temperature luminescence spectrum and corresponding circular polarization of luminescence for sample \#7. Pump wavelength $=750 \mathrm{~nm}$. 


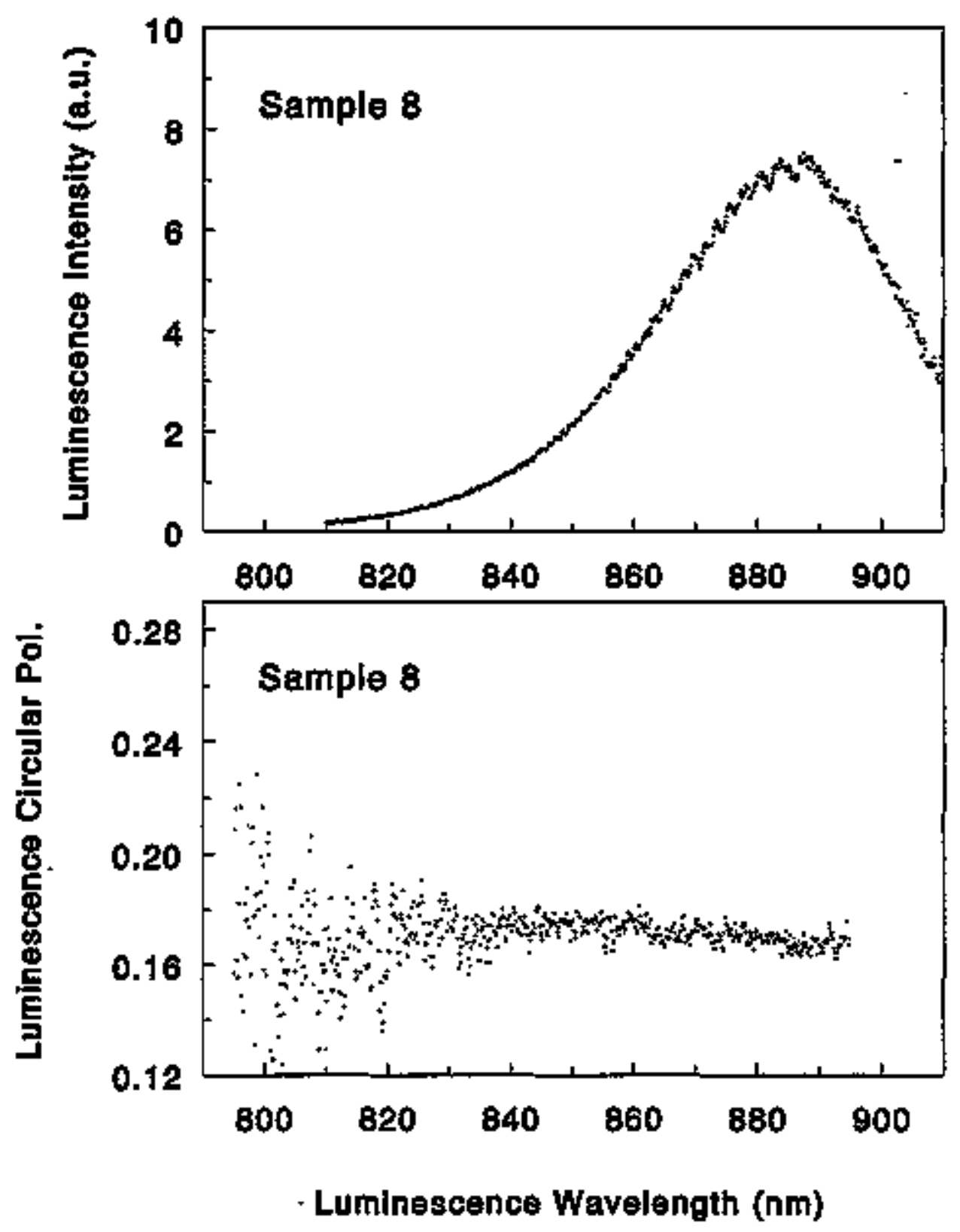

Figure 5.16: Room temperature luminescence spectrum and corresponding circular polarization of luminescence for sample \#8. Pump wavelength $=750 \mathrm{~nm}$. 


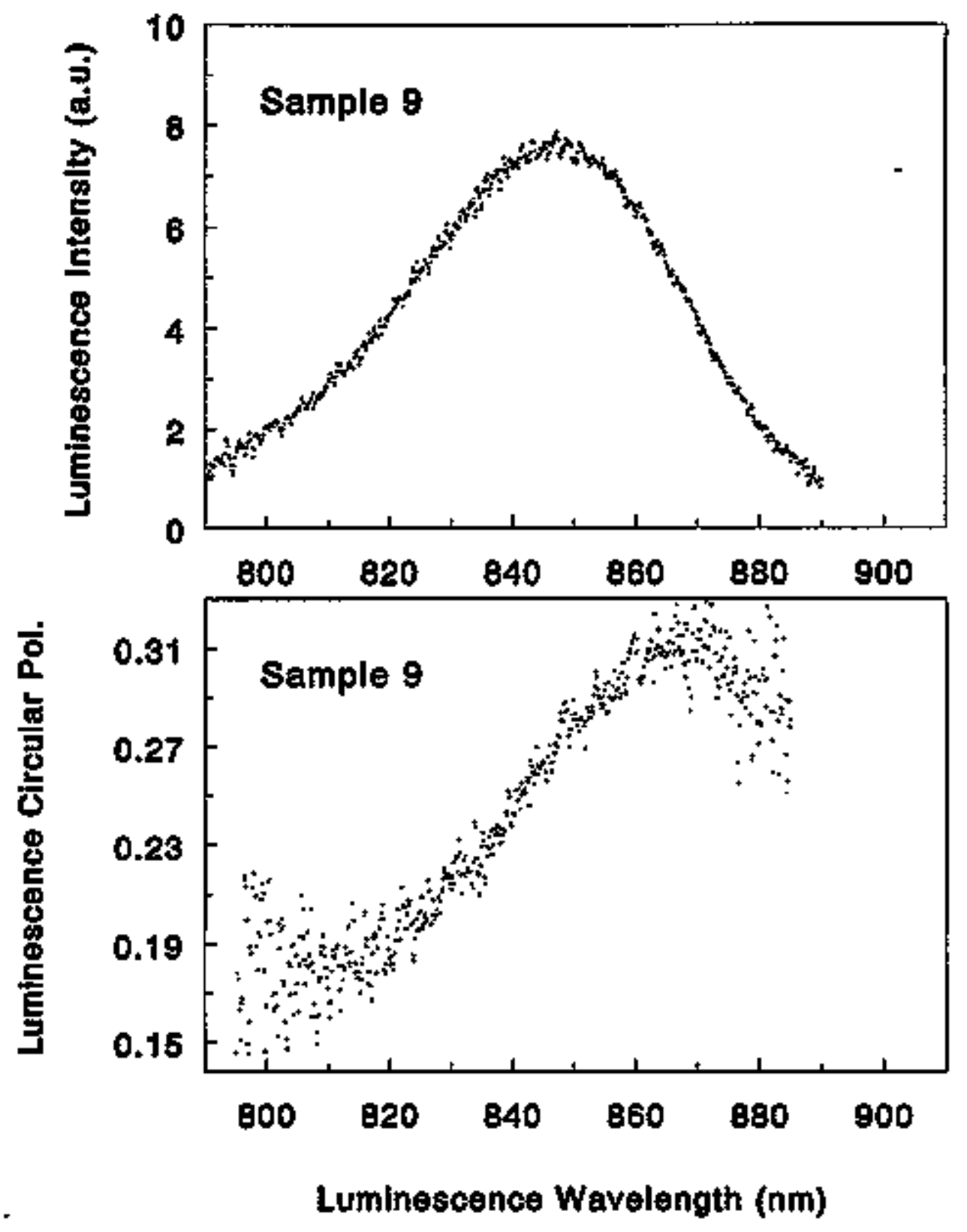

Figure 5.17: Room temperature luminescence spectrum and corresponding circular polarization of luminescence for sample \#9. Pump wavelength $=750 \mathrm{~nm}$. 
expected from the degree of strain measured by X-ray diffraction. This point will be discussed in Appendix B.

Another interesting aspect of the circular polarization curves becomes evident when viewed with their corresponding lurinescence spectra. As the degree of sample strain increases, so does the portion of the luminescence spectrum contributing to the enhanced level of circular polarization. For example, the highly strained sample \#1 shows an approximate twofold enhancement in luminescence polarization over the range of 800 to $850 \mathrm{~nm}$. The entanced polarization is achieved near the luminescence intensity maximum. The less highly strained sample \#6, on the other hand, only approaches such a degree of luminescence polarization enhancement in the long wavelength (low energy) tail of the luminescence spectrum. The relationship between the luminescence line-shape and the polarization for a given sample may be used as a measure of the degree of strain-induced splitting of the $m_{j}=3 / 2$ and $m_{j}=1 / 2$ valence bands. This correlation will be further developed and utilized in the following chapter to deconvolve the luminescence contributions of the two highest lying valence bands.

\subsubsection{Dependence on Pump Wavelength}

The dependence of luminescence circular polarization on the pump source wavelength was measured for each sample. Only a small portion of the luminescence spectrum from a given sample was observed and recorded during these measurements. The luminescence radiation passed tbrough an optical band-pass filter of nominally $10 \mathrm{~nm}$ pass-width. This filter was used to reject the pump laser radiation 
scattered from the sample surface in the direction of the spectrometer. Further rejection of the pump laser radiation was achieved by appropriate setting of the band-pass window which lies between the two spectrometers. Luminescence circular polarization measurements were then recorded for several pump wavelengths.

Figure 5.18 shows the luminescence spectra which were recorded for a typical polarization measurement under the condition of near-band-edge pump energy. The data were recorded for sample \#1 while pumping with $845 \mathrm{~nm}$ radiation. This is within $5 \mathrm{~nm}$ of the longest pump wavelength used successfully with this sample. Furthermore, the approximate $22 \mathrm{~mm}$ separation between pump wavelength and luminescence wavelength is among the smallest that was used for all measurements. In Figure 5.18, the filtered luminescence is seen at $867 \mathrm{~nm}$ while the unwanted pump laser light is seen at wavelengths less than $841 \mathrm{~nm}$. The laser wavelength is actually $845 \mathrm{~nm}$ and the apparent position and width of the laser line on the shown spectra are artifacts of the negative dispersion arrangement of the double spectrometer.

Because the luminescence polarization is not constant across the luminescence spectrum, measurement of the pump wavelength dependence on circular polarization required that the monitored luminescence wavelength range remain fixed for a given sample. It was necessary, however, to use two separate luminescence wavelength ranges for some samples in order to achjeve measurements at all of the desired pump wavelengths. When this was necessary, polarization measurements at both luminescence wavelength positions were made for some pump wavelengths such that a scale factor between the two luminescence wavelength positjons could 


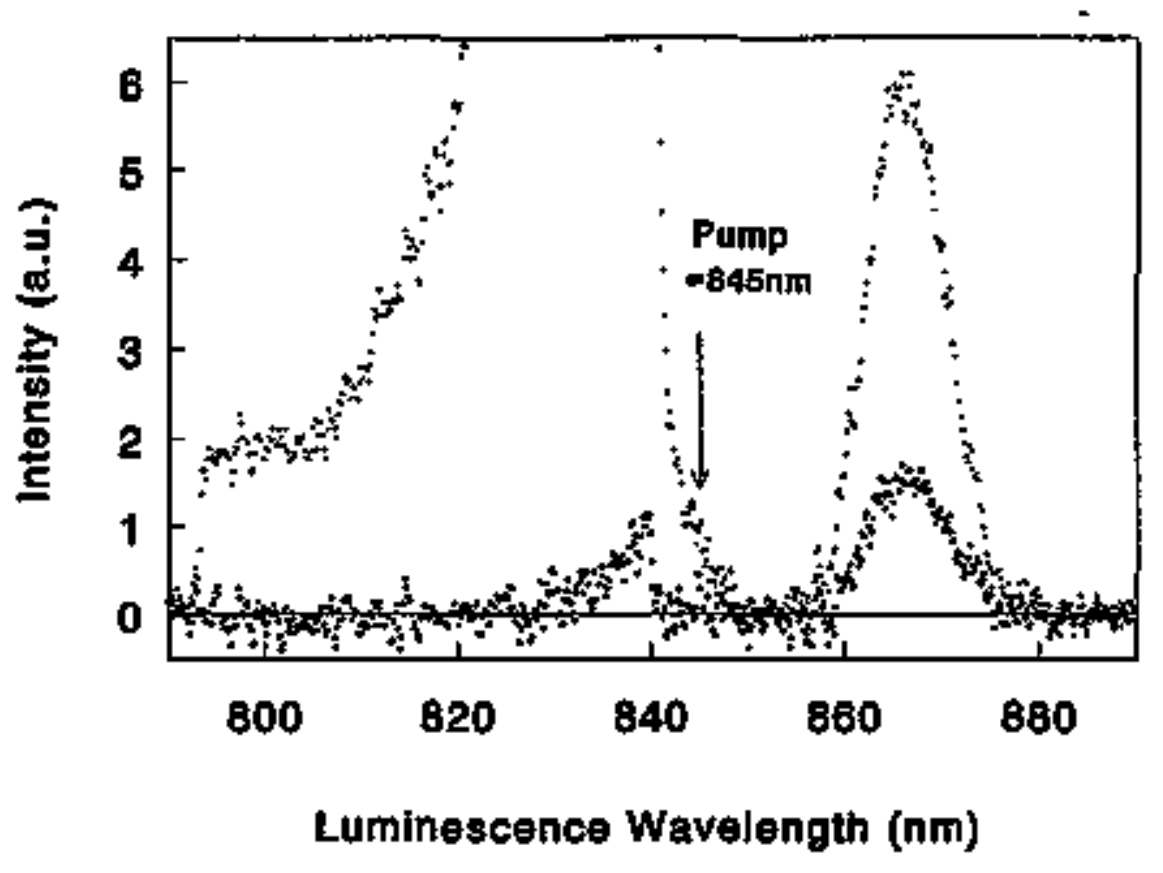

Figure 5.18: Example of spectra taken for a measurement of luminescence circular polarization as a function of punp wavelength. The spectra were recorded for sample \#1 while pumping with $845 \mathrm{~nm}$, and the spike-filtered luminescence is seen at $867 \mathrm{~nm}$. The larger luminescence peak represents the sum of (pump/lum) $\sigma^{+} / \sigma^{-}$and $\sigma^{-} / \sigma^{+}$acquisitions, while the other represents the sum of $\sigma^{+} / \sigma^{+}$and $\sigma^{-} / \sigma^{-}$acquisitions. 
be determined. The scale factors were determined by a least squares fit of the wavelength-overlapped data.

The data presented here have some systematic and statistical uncertainties. The leading source of uncertainty for most of the data is false asymmetries introduced by beam steering under rotation of the $\lambda / 4$ retarders. These false asymmetries are believed (conservatively) to be smaller than $0.5 \%$ absolute. Some of the data taken at the longest pump wavelengths for a given sample have the smallest luminescence signals and statistical errors on the order of $1 \%$ absolute, but the majority of the data bas negligible statistical uncertainty. False asymmetries may also be introduced by stray laser light contamination of the luminescence. Several attempts were made to measure any such false asymmetry with the result of no evidence for values significantly larger than the $0.5 \%$ quoted for false asymmetries due to steering.

The circular polarization for each of the samples is plotted against pump wavelength in Figures 5.19 through 5.27. The luminescence wavelength that the measured polarizations pertain to is indicated for each plot. Also plotted with the data are the luminescence wavelength dependent circular polarization curves which were shown in Figures 5.9-5.17. The wavelength scale of the plots corresponds to pump source wavelength for the discrete dats points and to luminescence wavelength for the contisuous curves. For each sample, the two polarization axes are scaled to provide the best visual fit. The good agreement observed between the wavelength dependence of each type of measurement is a confirmation of the expected equivalence of $P_{e-}$ and $P_{h}$. It is also an indication that $R$ is independent of both pump 
and luminescence photon energy.

The plot for sample \#6 (Fig. 5.24) shows some apparent disagreement between the two types of measurement. Additional data was acquired to check for a possible error in the original data which might explain the apparent disagreement, but no such error was found. The measurements taken for purnp wavelengths less than $820 \mathrm{~nm}$ and those taken for pump ravelengths longer than $860 \mathrm{~nm}$ both use a luminescence wavelength of $840 \mathrm{~nm}$ so that no scale factor is required. The time of polarization measurement in relation to sample history could possibly affect the final data. The data taken for pump wavelengths longer than $860 \mathrm{~nm}$ was acquired at a time after the sample had been cooled to $\sim 12 \mathrm{~K}$ while the measurements for wavelengths less than $820 \mathrm{~nm}$ were taken prior to that time. It may be possible that the handling and cooling of the sample between the two sets of measurements had an effect which lowered the observed luminescence polarization slight]y. However, this possibility is not confirmed in any conclusive way.

\subsection{Comparison of Luminescence Circular Polarization with Photo-Emitted Electron Spin Polarization}

The photocathode materials used in this study were also used in photo-emission experiments to measure electron spin polarization directly. The photo-emission experiments are part of the SLAC/Wisconsin photocathode research and development program. The photocatbodes were activated and operated in the PEGGY Il Polarized Electron Gun and the photo-emitted electron spin polarization was analyzed with a calibrated Mott polarimeter. The details of the PEGGY II system 


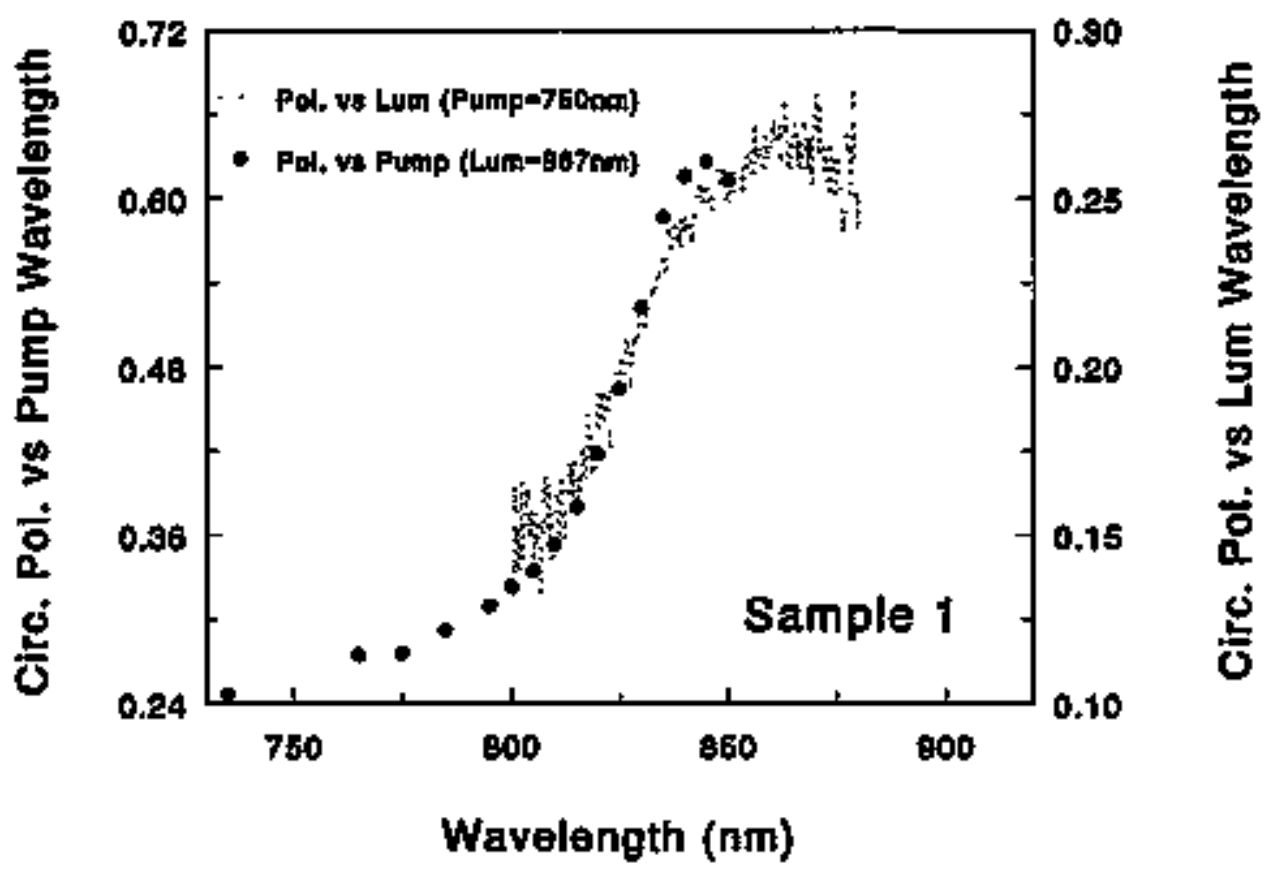

Figure 5.19: Room temperature pump wavelength dependence of luminescence circular polarization for sample \#1 shown with the luminescence wavelength de pendent polarization curve from Figure 5.9. 


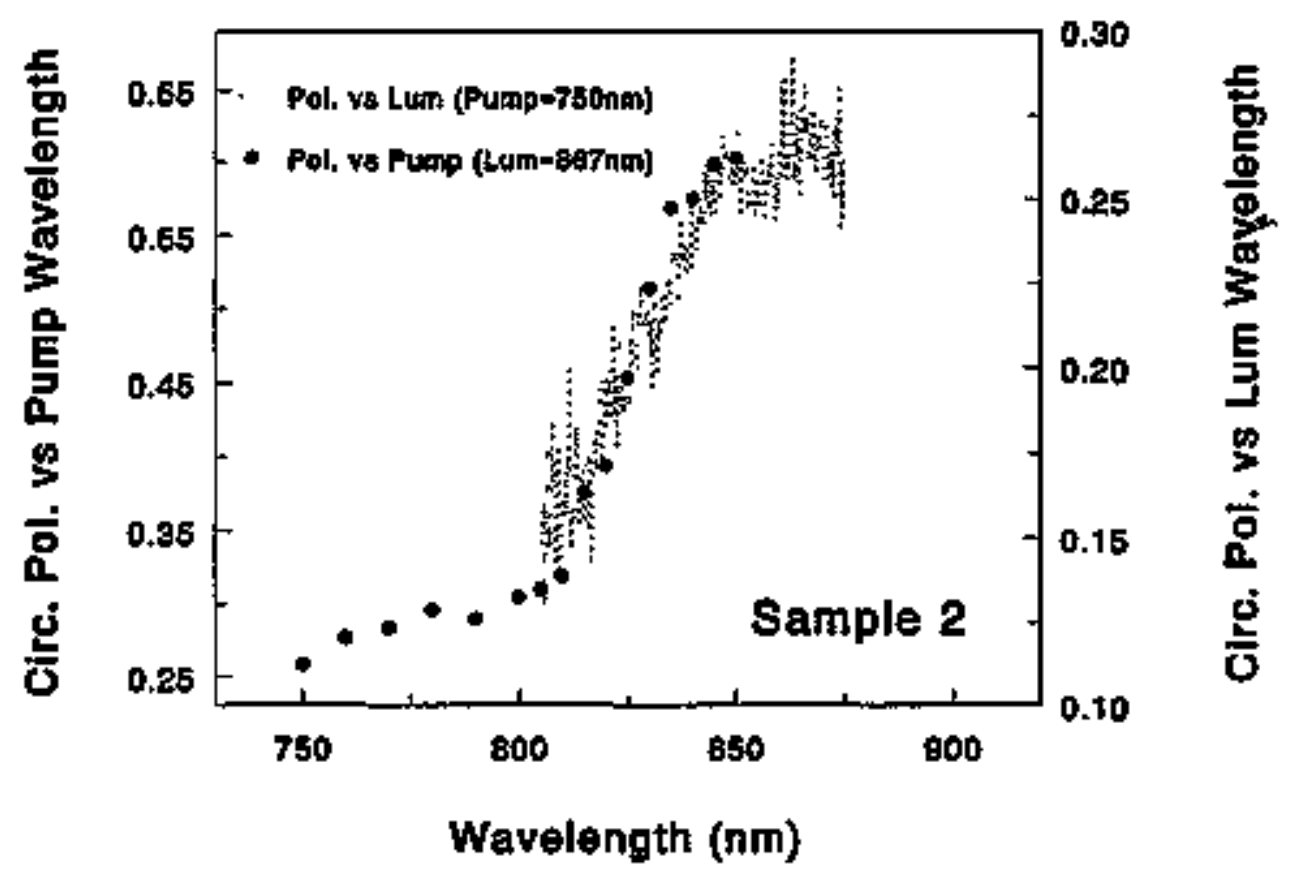

Fjgure 5.20: Room temperature pump wavelength dependence of luminescence circular polarization for sample \#2 shown with the luminescence wavelength dependent polarization curve from Figure 5.10. 


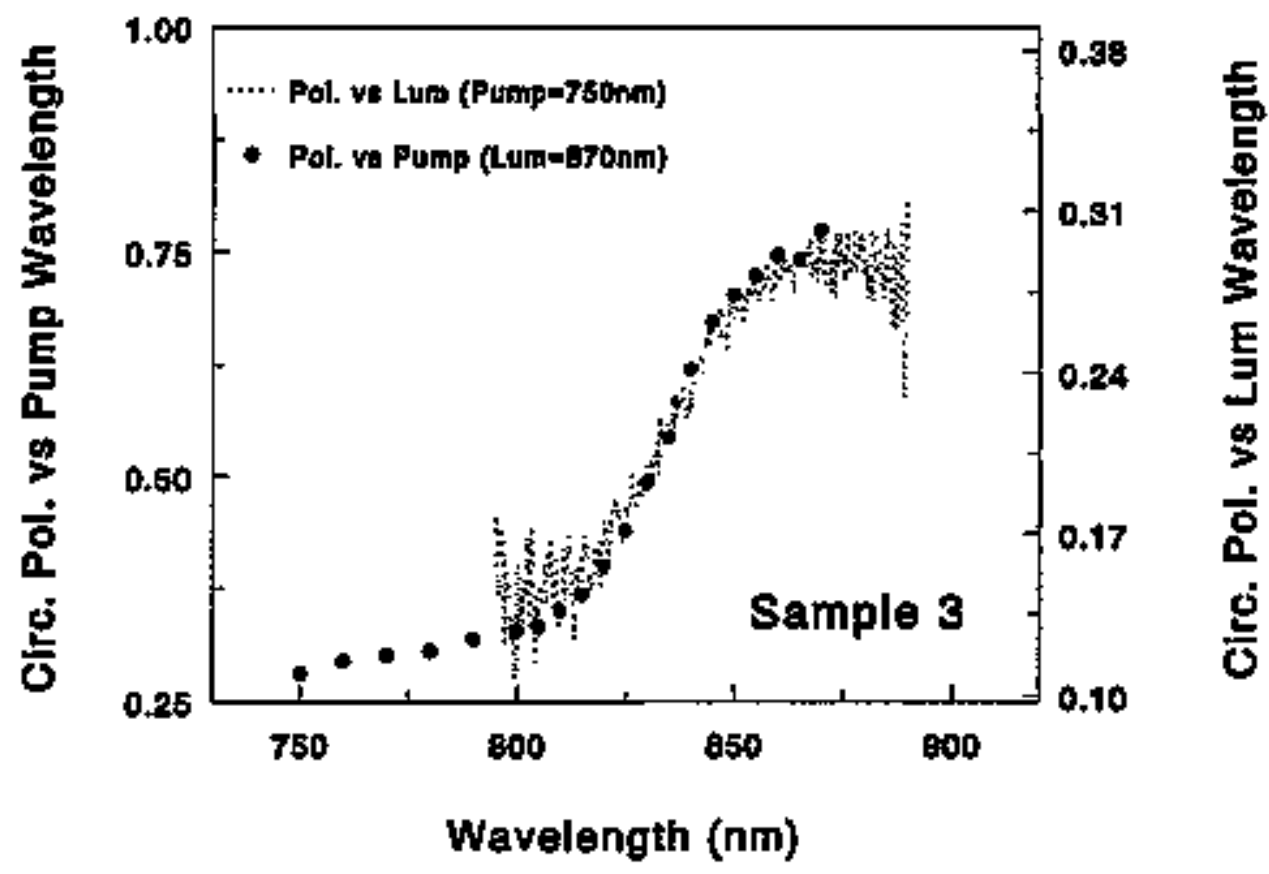

Figure 5.21: Room temperature pump wavelength dependence of luminescence circular polarization for sample \#3 shown with the luminescence wavelength dependent polarization curve from Figure 5.11. 


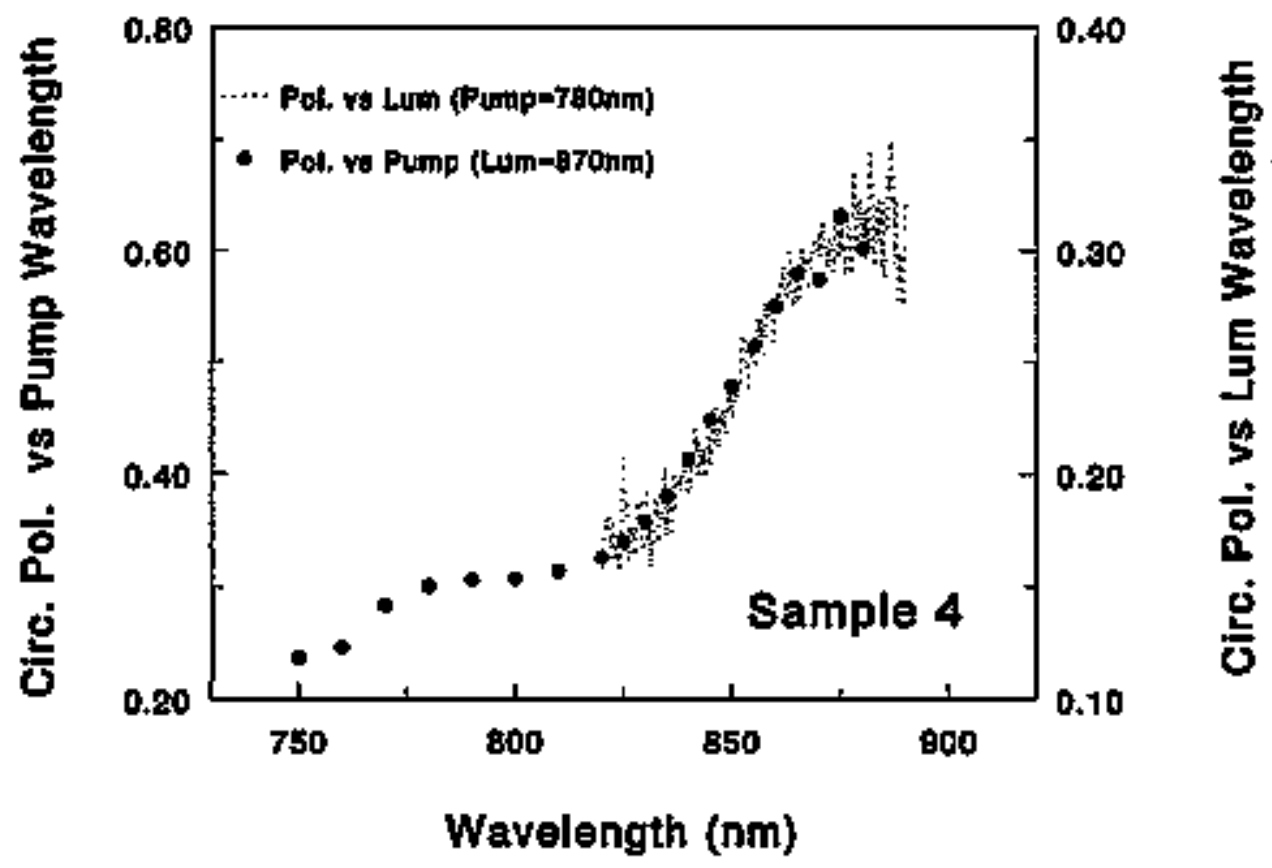

Figure 5.22: Room temperature pump wavelength dependence of luminescence circular polarization for sample \#4 shown with the luminescence wavelength dependent polarization curve from Figure 5.12 . 


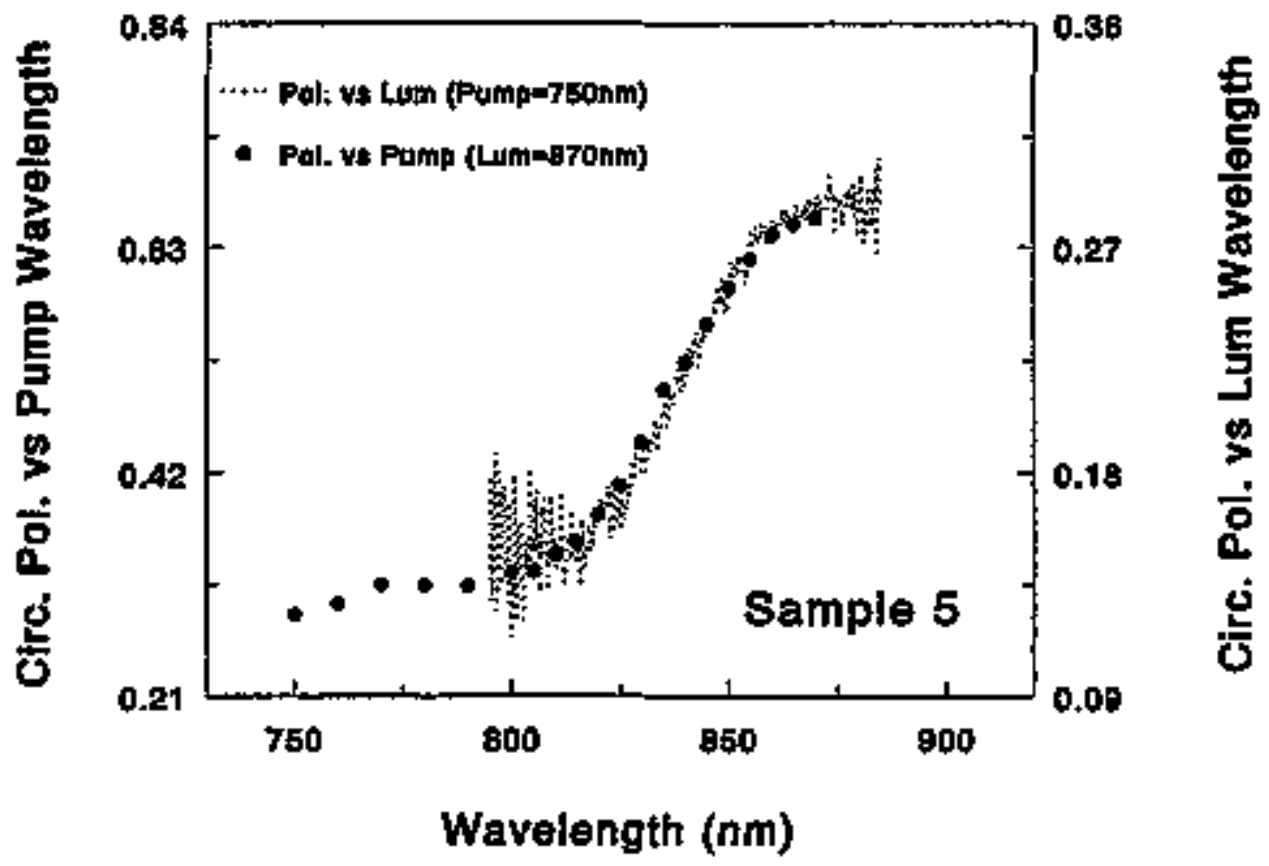

Figure 5.23: Room temperature pump wavelength dependence of luminescence circular polarization for sample \#5 shown with the lominescence wavelength dependent polarization curve from Figure 5.13 . 


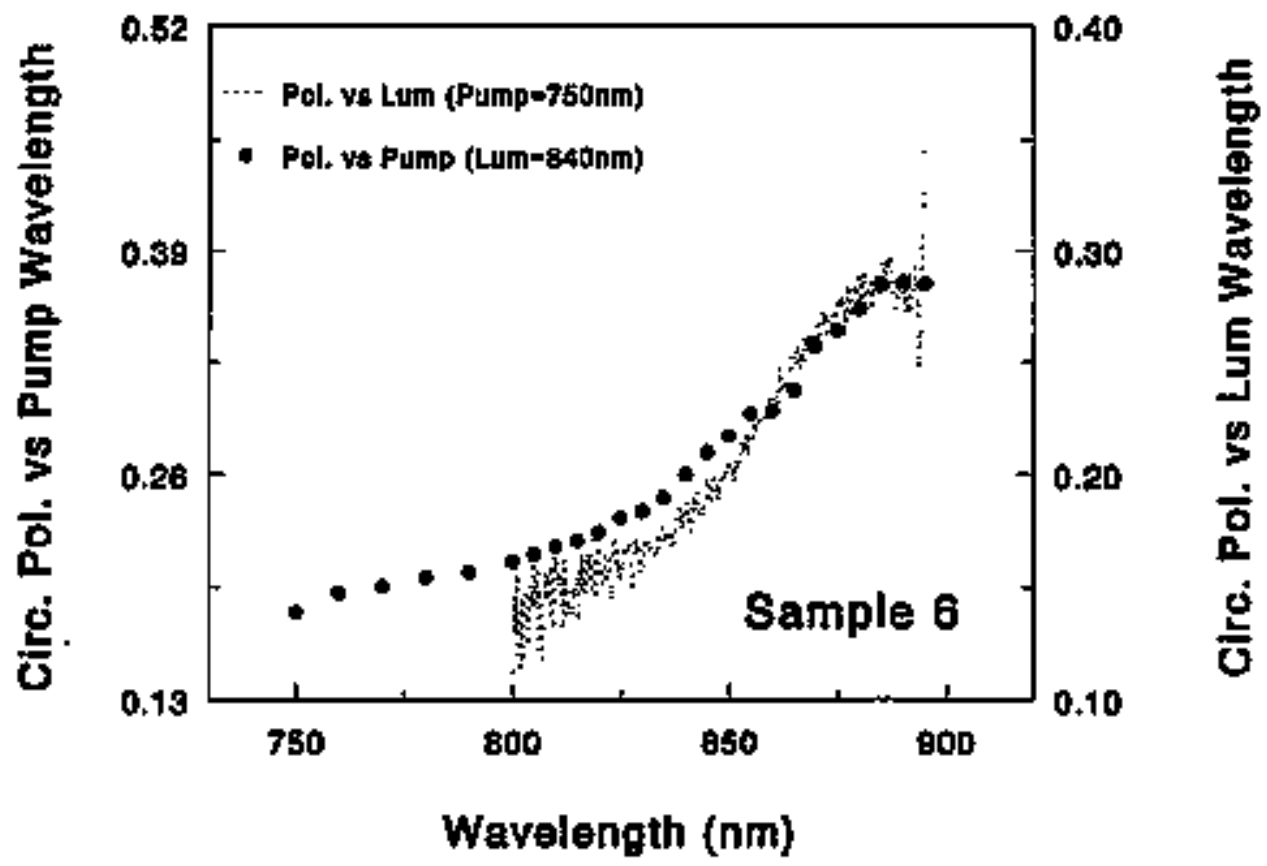

Figure 5.24: Room temperature pump wavelength dependence of luminescence circular polarization for sample \#6 shown with the luminescence wavelength dependent polarization curve from Figure 5.14. 


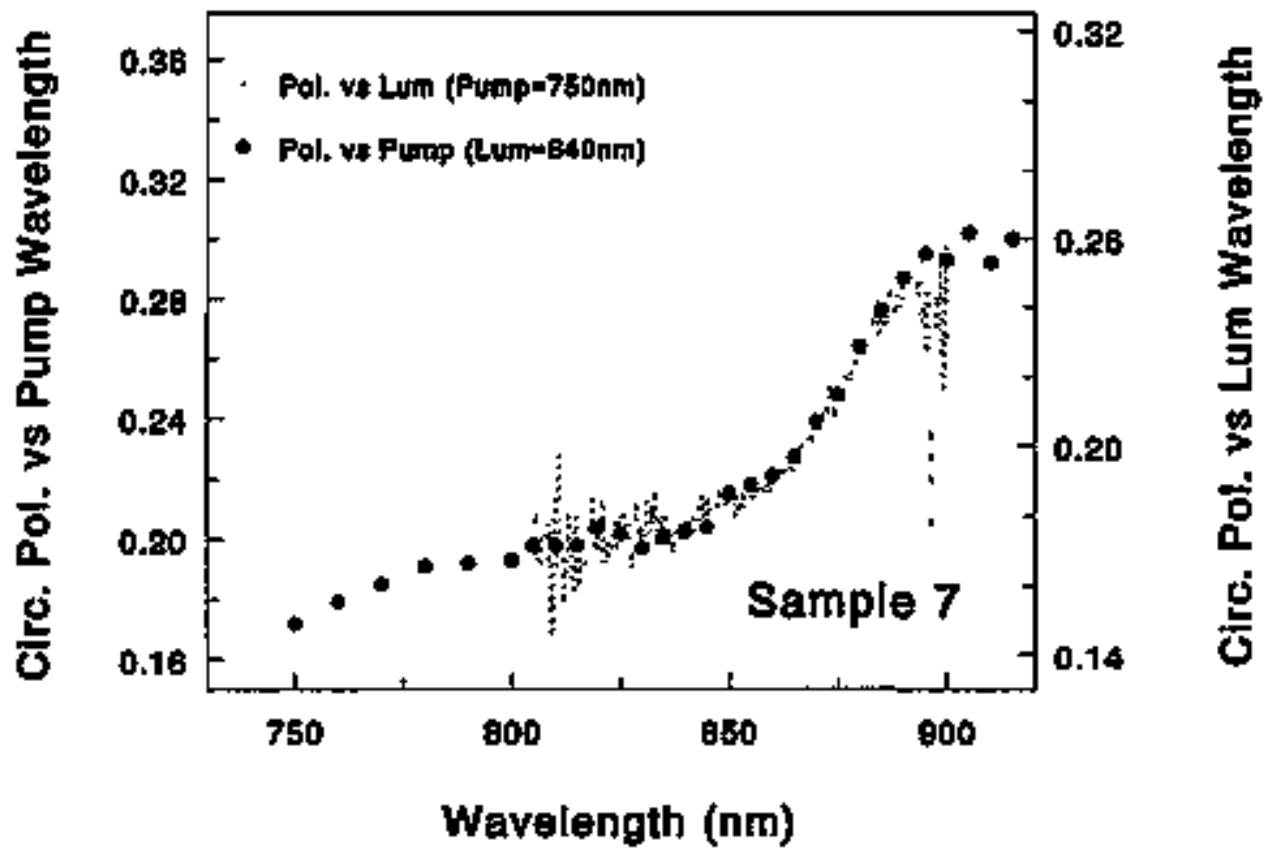

Figure 5.25: Room temperature pump wavelength dependence of luminescence circular polarization for sample \#7 shown with the luminescence wavelength dependent polarization curve from Figure 5.15 . 


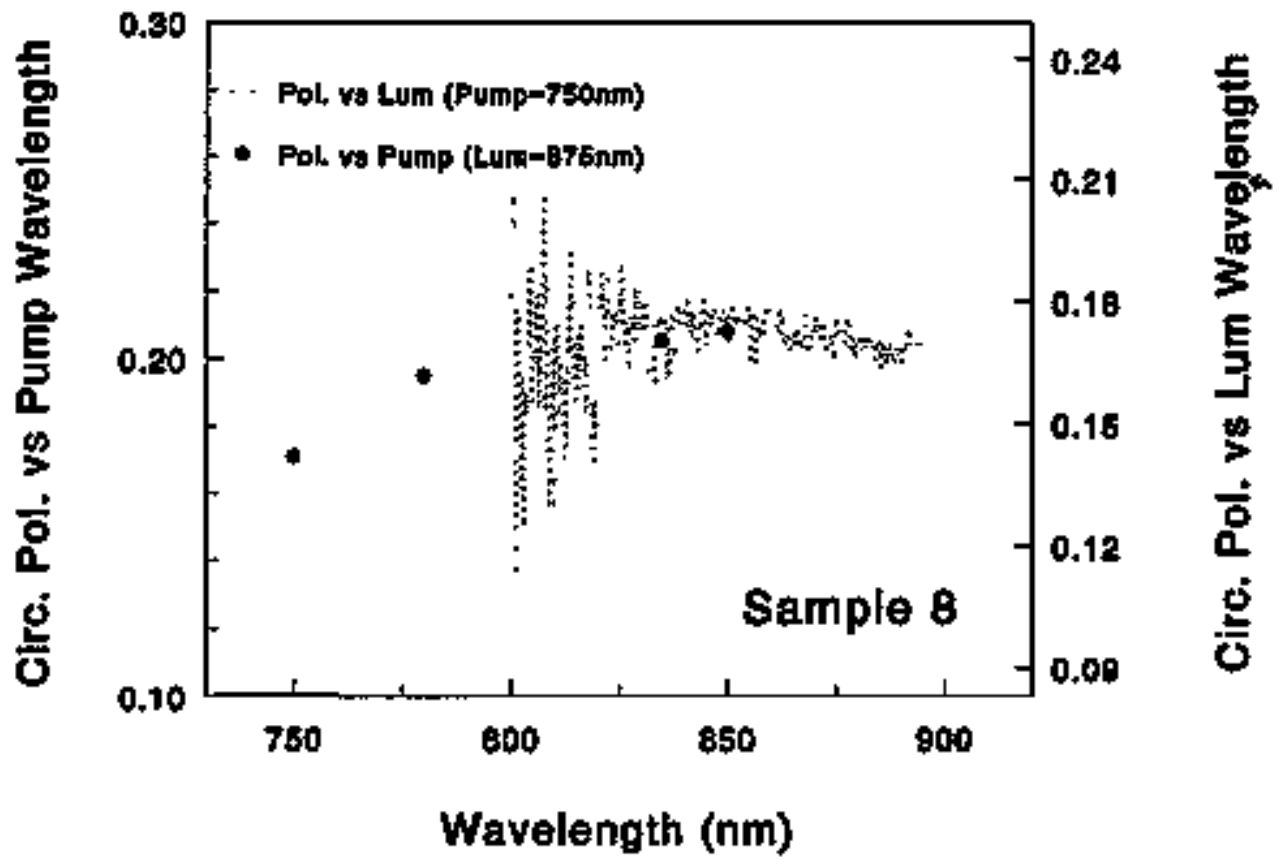

Figure 5.26: Room ternperature pump wavelength dependence of luminescence circular polarization for sample \#8 shown with the luminescence wavelength dependent polarization curve from Figure 5.16. 


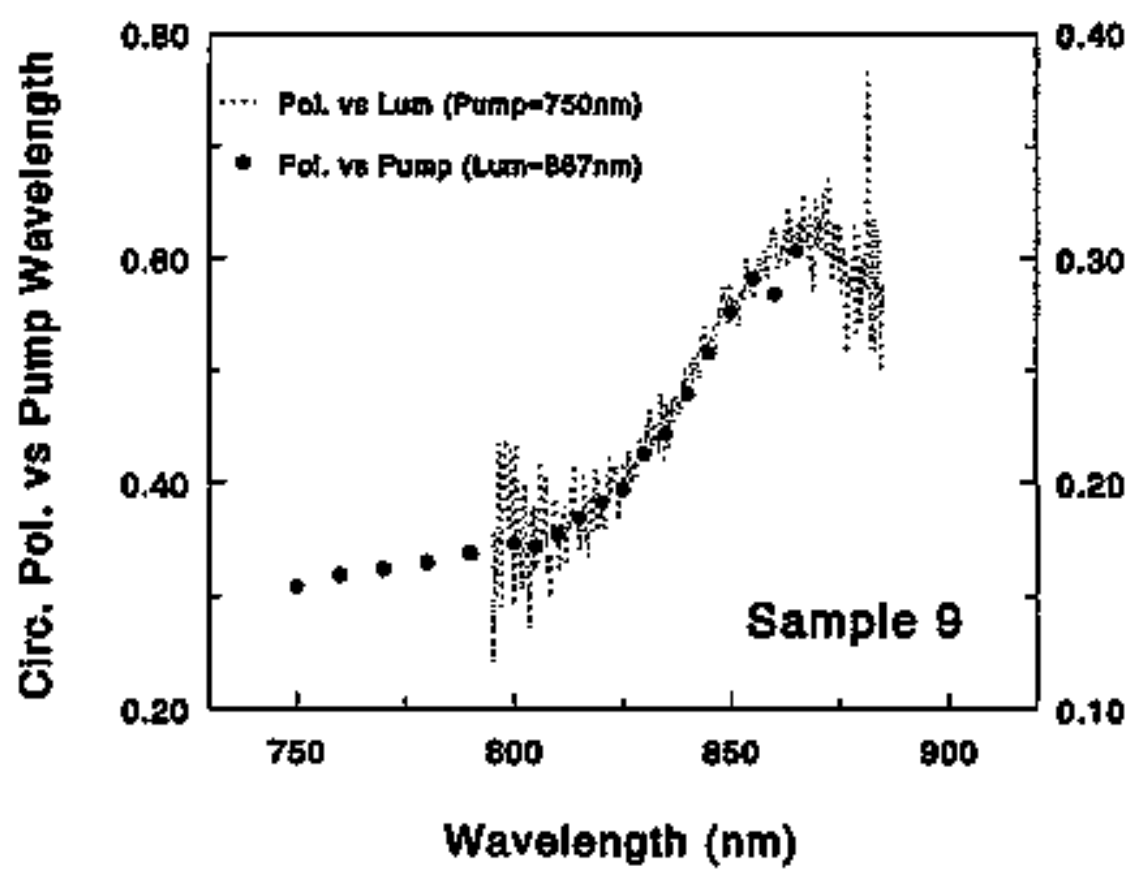

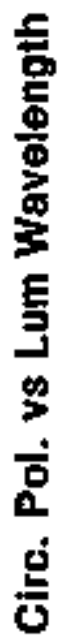

Figure 5.27: Room temperature pump wavelength dependence of luminescence circular polarization for sample \#9 shown with the luminescence wavelength dependent polarization curve from Figure 5.17. 
are described elsewhere. [66]

Spin polarization measurements obtained with the PEGGY II system were available for samples \#1 through \#8. It should be noted that these measurements were performed independently of the present stady. In some cases the mensurements were performed on the actual samples used in this photoluminescence study, while in other cases the measurements were performed on separate samples cut from the same photocatbode wafers. The spin polarization measurements are estimated to have a systematic uncertainty of approximately $5 \%$ relative.

Some concern existed regarding the accuracy of the wavelength scale for the pump laser used with the PEGGY II system. The data from individual samples, when compared with luminescence data and spin polarization data from a second polarimeter system at SLAC[67], was found to be consistent with an offset in wavelength of approximately $10 \mathrm{~nm}$. Therefore, the spin polarization data is presented with a positjve $10 \mathrm{~nm}$ offset added to the wavelength scale.

Figures 5.28 through 5.35 show the electron spin polarization data plotted with the corresponding luminescence circular polarization data. The spin polarization and luminescence polarization axes are scaled to provide a minimum (rms) deviation over common wavelength data points in the region of pump wavelengths indicated by vertical dashed lines. A horizontal dashed line on each of the plots indicates the approximate maximum luminescence circular polatization levels. These levels are taken from Figures 5.19 through 5.27. Figures 5.28, 5.29 and 5.31 show that the maximum scaled luminescence polarization is consistent with a $100 \%$ maximum spin polarization value $\left(P_{r}\right.$ (scaled $\left.) \geq 97.5 \%\right)$. Also observed in Figures 5.28, 


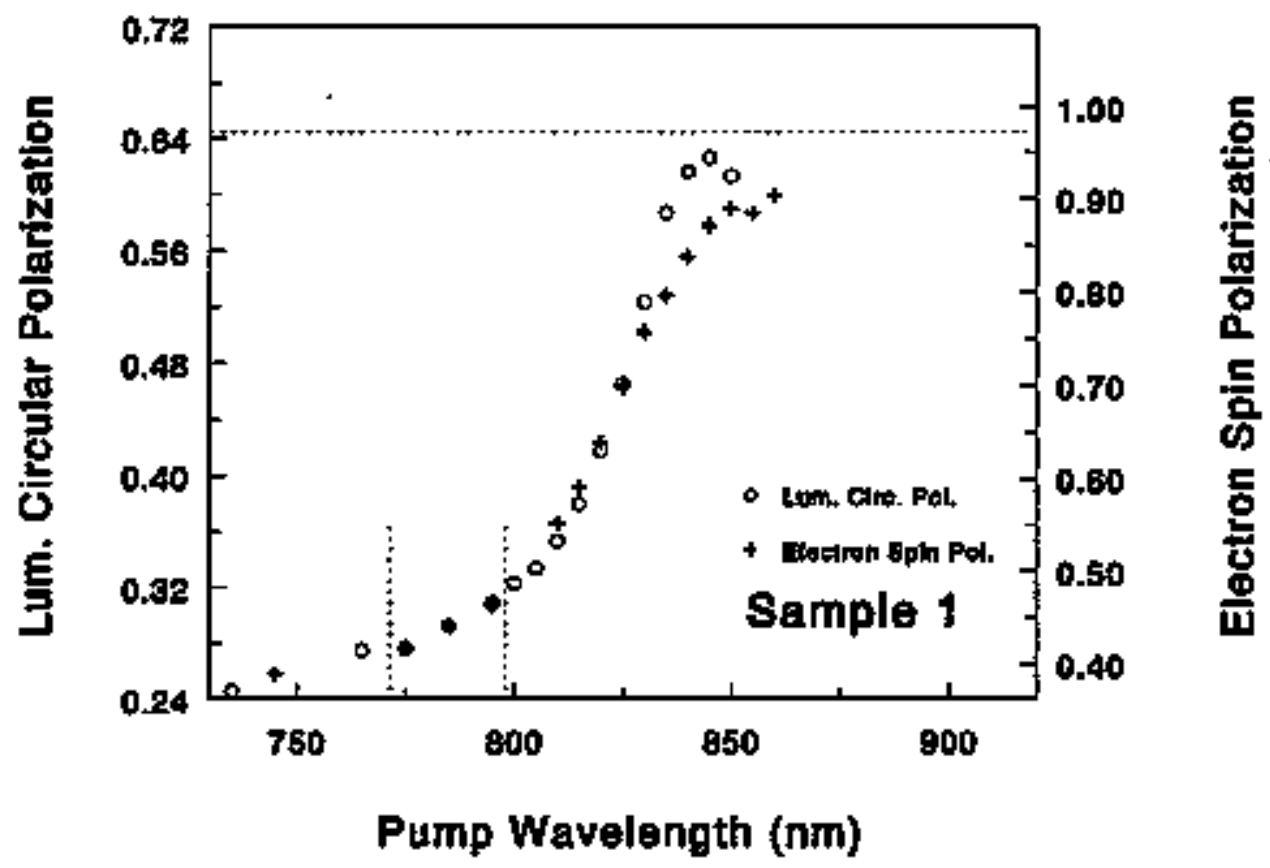

Figure 5.28: Pump wavelength dependent electron spin polarization (from photo-mission) and luminescence circular polarization for sample \#1 at room temperature. 


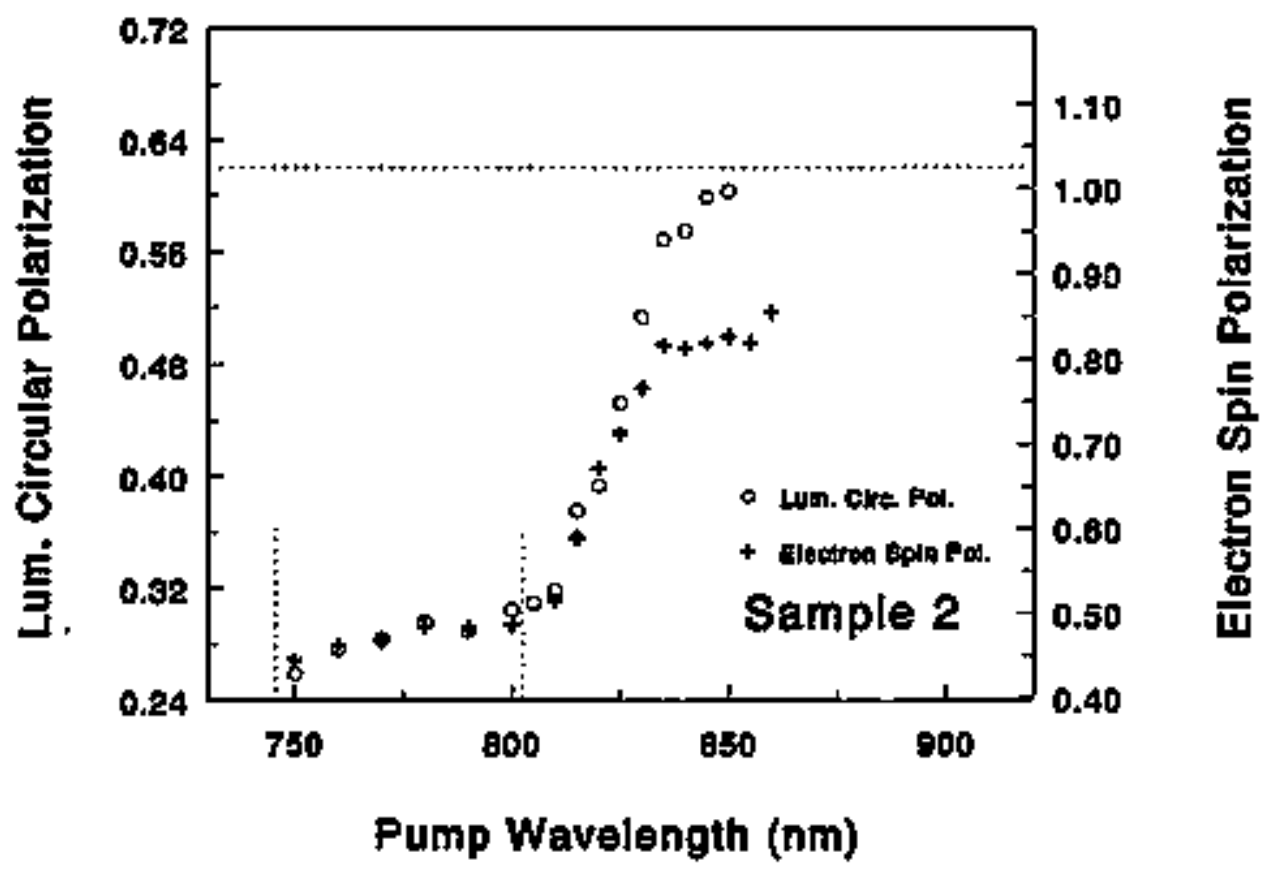

Figure 5.29: Pump wavelength dependent electron spin polarization (from photo-emission) and luminescence circular polarization for sample \#2 at room temperature. 


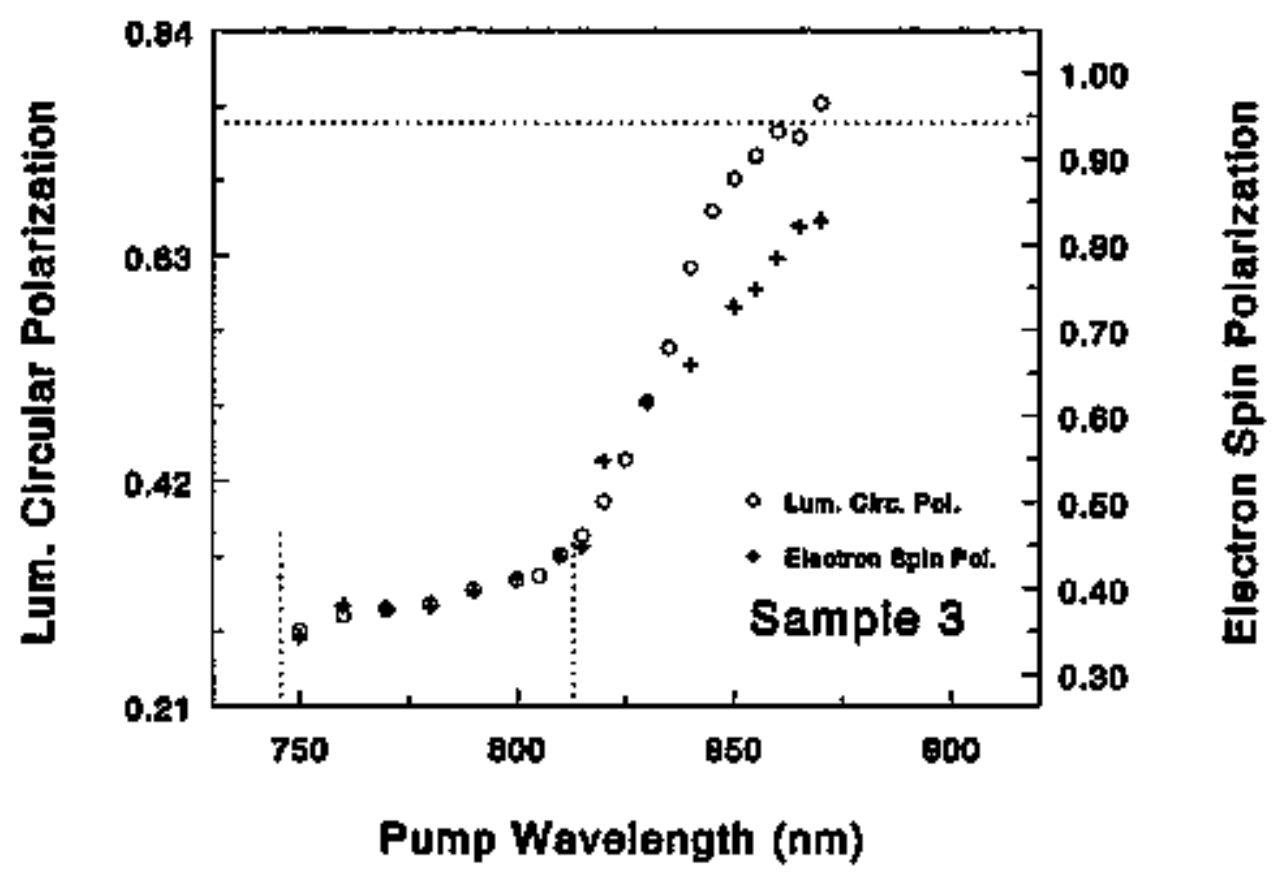

Figure 5.30: Pump wavelength dependent electron spin polarization (from photo-emission) and luminescence circular polarization for sample \#3 at room temperature. 


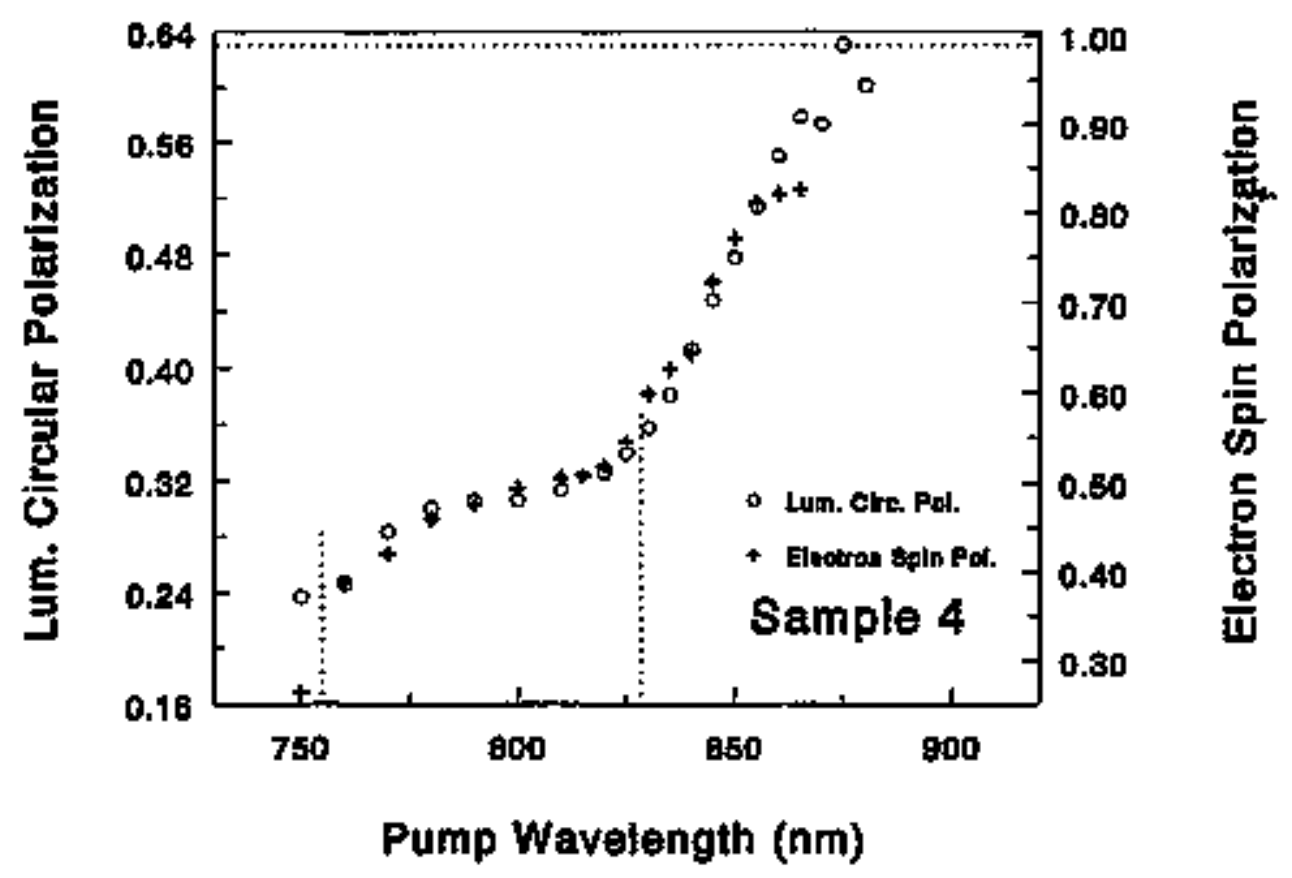

Figure 5.31: Pump wavelength dependent electron spin polarization (from photo-emission) and luminescence circular polarization for sample \#4 at room temperature. 


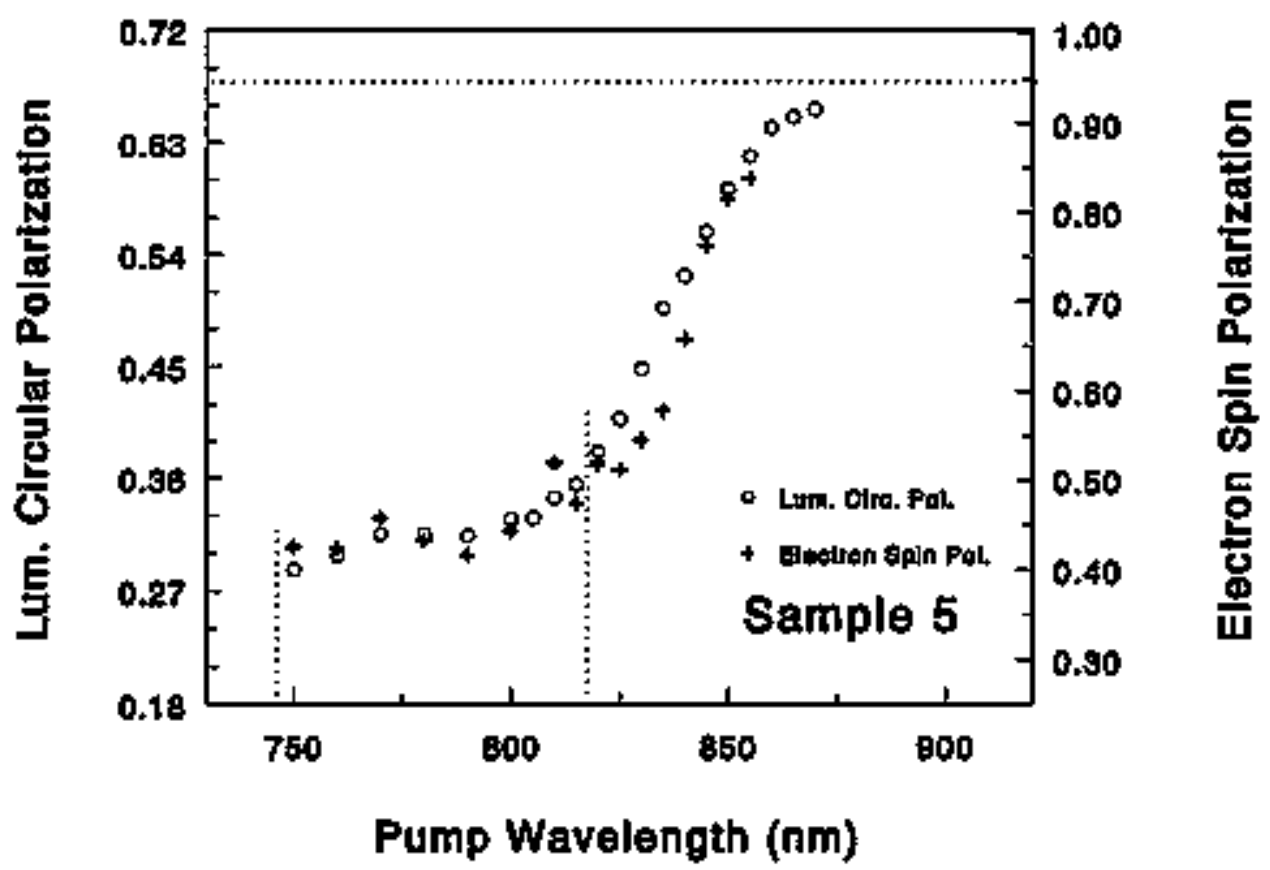

Figure 5.32: Pump wavelength dependent electron spin polarization (from photo-emission) and luminescence circular polarization for sample $\# 5$ at room temperature. 


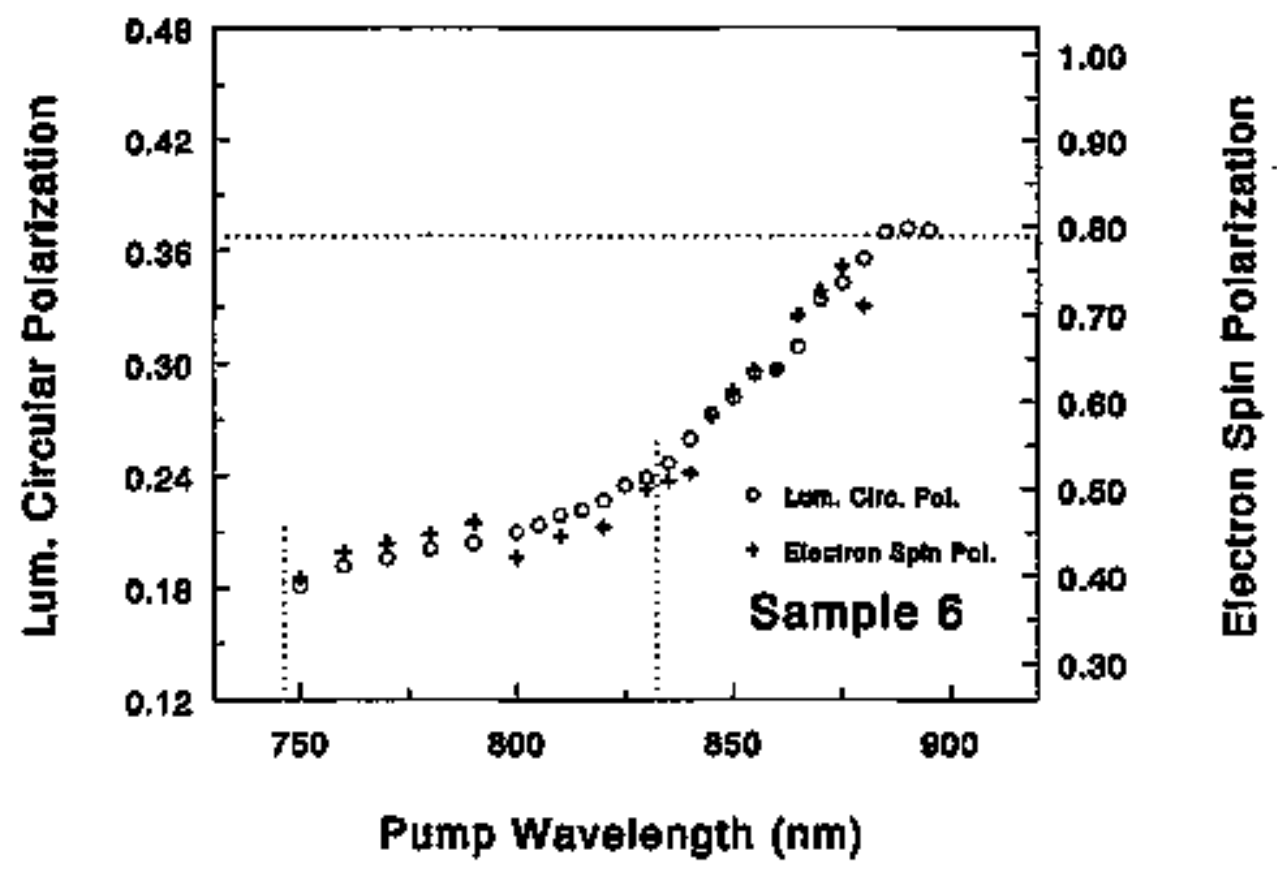

Figure 5.33: Pump wavelength dependent electron spin polarization (from photo-enission) and luminescence circular polarization for sample \#6 at room temperature. 


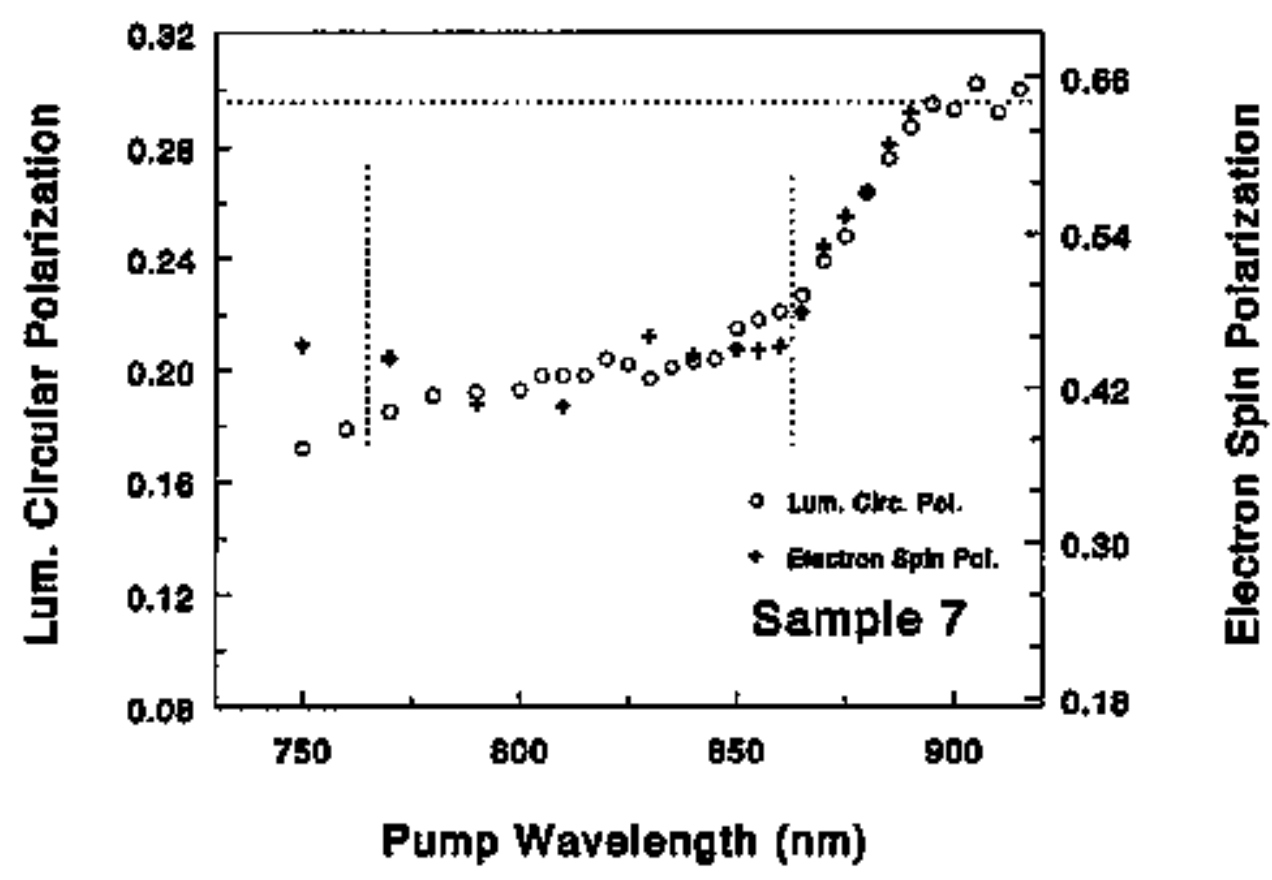

Figure 5.34: Pump wavelength dependent electron spin polarization (from photo-emission) and luminescence circular polarization for sample \#7 at room temperature 


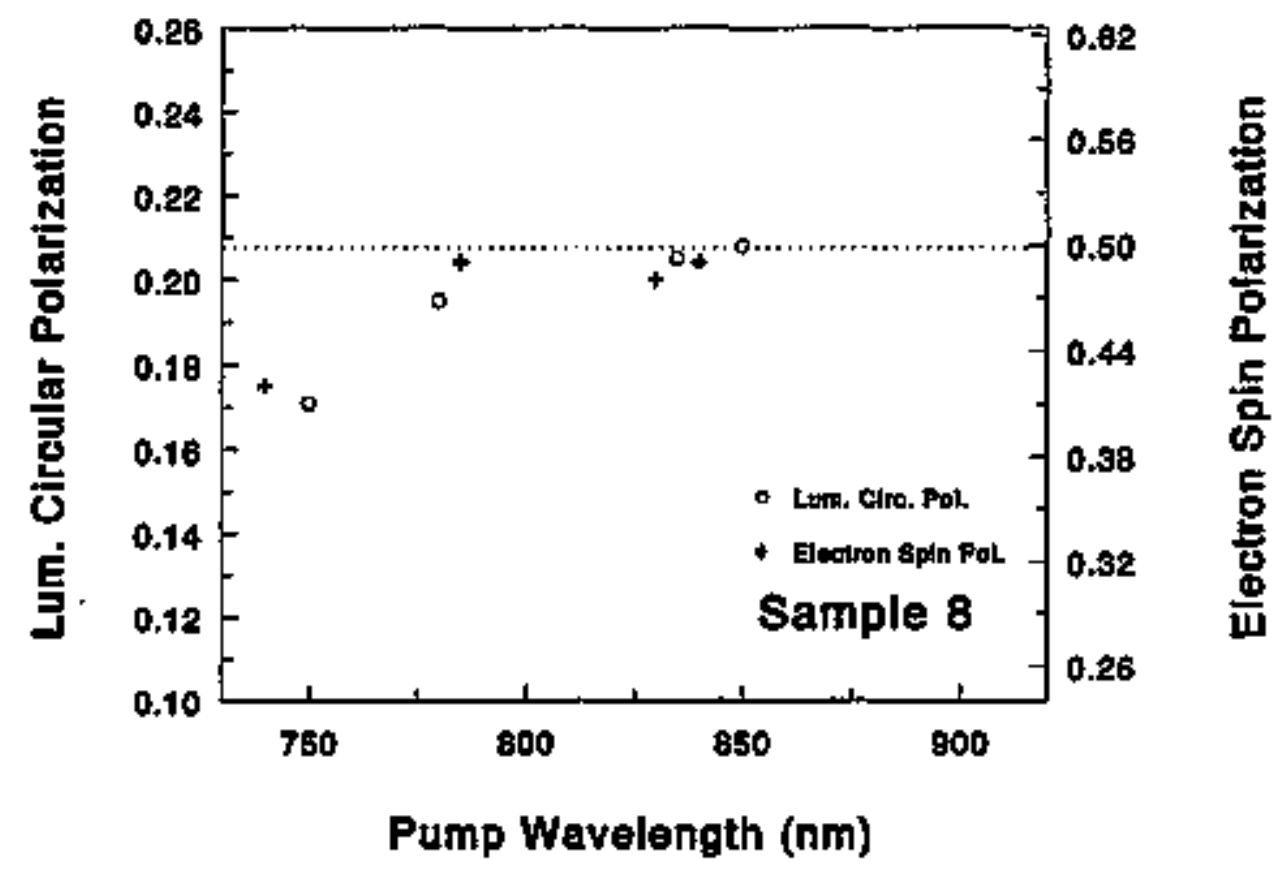

Figure 5.35: Pump wavelength dependent electron spin polarization (from photo-emission) and luminescence circular polarization for sample \#8 at room temperature. 
5.29 and 5.31 is that the 'knee' where the polarization is to begin increasing strongly with increasing wavelength is typically in the vicinity of $50 \%$ electron spin polarization, such that there is a twofold polarization enhancement from the 'knee' to the maximum level. The same twofold enhancement in luminescence circular polarization is seen for samples \#3 and \#5 in Figures 5.30 and 5.32. In these figures though, the maximum luminescence polarization is consistent with an electron spin polarization of only approximately $94-95 \%$. It is argued that, for these samples, the twofold increase in luminescence polarization from the 'knee' to the maximum is an indication that the maximum electron spin polarization at the time of the optical transitjon does reach $100 \%$, and the less than $100 \%$ (scaled) value observed is an jndication of a spin depolarization factor associated with the electron photo-emission process for these samples. On the other hand, the data for samples \#6 and \#7 shown in Figures 5.33 and 5.34 do not show a twofold enbancement in luminescence circular polarization from the 'knee' to the maximum. This is interpreted as an indication that the electron spin polarization at the time of the optical transition never reaches the level of $100 \%$ because the situation of parely selective optical pumping from only the $\left|\frac{3}{2}, \frac{3}{2}\right\rangle$ valence band is never reached. Rather, some level of optical transitions from the $\left(\frac{3}{2}, \frac{1}{2}\right)$ valence band persist at even the longest pump wavelengths. The final figure, Fig. 5.35, shows data for the non-strajned GaAs sample. Because of the limited number of data. points for this sample, the scaling of luminescence polarization to electron spin polarization was simply chosen to provide a reasonable visual fit. It is seen in Figure 5.35 that the maximum luminescence circular polarization corresponds 
roughly to the maximum expected electron spin polarization of $50 \%$.

\subsection{Luminescence Measurements at Low Temperatures}

In addition to the room temperature data, luminescence measurements were made at $\approx 78 \mathrm{~K}$ for all of the samples used in this study. This data was acquired primarily to jnvestigate the possibility of a temperature dependence on luminescence circular polarization for the various samples. Some of the samples were also cooled to $\approx 12$ $\mathrm{K}$ with liquid $\mathrm{He}$ to further investigate any possible temperature dependent effects. As discussed in Section 4.4, the measured temperatare with the liquid He filled dewar is expressed as $T \approx 12 \mathrm{~K}$ for all samples, even though the temperature varjed from $\sim 8$ to $\sim 13 \mathrm{~K}$ over the set of samples.

The luminescence spectra for all samples will shift to shorter wavelengths (larger energy) upon cooling. This is due to the temperature dependence of the band gap for GaAs given by[68]

$$
E_{g}(T)=E_{g}(0)-\frac{5.405 \times 10^{-4} T^{2}}{204+T},
$$

where temperature $T$ is expressed in Kelvin. From eq. (5.13), a shift in band gap of $83.5 \mathrm{meV}$ is expected for the GaAs samples upon cooling from room temperature $(\approx 297 \mathrm{~K})$ to $\approx 78 \mathrm{~K}$. It is observed as a shift of the luminescence peak position and the wavelength where strain enhancement of luminescence circular polarization occurs. Similarly, upon cooling to $\approx 12 \mathrm{~K}$, a ehift of $94.8 \mathrm{meV}$ is expected. Another expected temperature dependent effect is the narrowing of the luminescence spectra at low temperatures. This is due to both a somewhat decreased 
level of band tailing and a smaller $k_{B} T$ spread in the Fermi-statistical distrjbution. Finally, a smaller degree of band tailing and reduced $k_{B} T$ spread at low temperatures is expected to result in a more rapid enhancenent in iuminescence circular polarization as the pump photon energy is decreased and transitions from the $\left(\frac{3}{2}, \frac{1}{2}\right)$ valence band are suppressed. In fact, this may also result in a higher obtainable degree of electron spin polarization in the less strained samples due to a larger obtainable ratio of transitions from the $\left(\frac{3}{2}, \frac{3}{2}\right)$ valence band to those from the $\mid \frac{3}{2}, \frac{1}{2}$ ) valence band.

Using luminescence peak positions from spectra recorded at $\approx 78 \mathrm{~K}$ and those found at room temperature, a peak shift of $69.1 \pm 3.9 \mathrm{meV}$ was observed for the samples. The value given is an average over the samples and the uncertainty is the standard deviation over the samples. The peak shift observed upon cooling to $\approx 12 \mathrm{~K}$ was $72.6 \pm 2.7 \mathrm{meV}$. These shifts are smaller than the band gap shifts expected from eq. (5.19), but this is not surprising if one considers the temperature dependence of the relationship between band gap energy and luminescence peak energy. The luminescence peak position relative to the band gap minimum is related to the band tailing present and the Fermi-statistical distributions within the conduction and valence bands. At room temperature, the distributions are broader than at low temperature, resulting in a wider luminescence spectrum and a peak energy further from band edge. A more accurate correspondence between the luminescence spectann and the temperature dependent band gap is found if one measures the luminescence position from an extrapolation of the low energy side of the spectrum. [69] 


\subsubsection{Measurements at $78 \mathrm{~K}$}

Luminescence circular polarization measurements were made at $78 \mathrm{~K}$ for all of the samples used in this study. The method of measurement was the same as described for room temperature measurements. Figure 5.36 shows the luminescence spectrum and corresponding luminescence wavelength dependent polarization curve for sample \#4 at $78 \mathrm{~K}$. The room temperature luminescence spectrum and polarization curve are also shown in Figure 5.36 for comparison. As expected, the 78 $\mathrm{K}$ luminescence spectrum is narrower than the room temperature spectrum. In Figure 5.36, an approximate twofold enhancement in luminescence circular polarization is seen over the wavelength range of 795 to $825 \mathrm{~nm}$ for the $78 \mathrm{~K}$ curve, while the twofold enhancement for the room temperature curve occurs over the wavelength range of 820 to $880 \mathrm{~nm}$. Thus, the expected shift and sharpening of the luminescence circular polarization curve at lower temperature are both observed.

Samples with strains larger than that of sample \#4 did not show $78 \mathrm{~K}$ luminescence polarization curves with the structure (twofold enkancement) seen in Figure 5.36, while those possessing a lesser degree of strain did show similar structure. This is believed to be due simply to some level of contamination in the luminescence spectrum from either scattered laser light or luminescence from other structures within the sample. The highly strained samples are expected to show the lurninescence polarization structure further into the high energy tails of the luminescence spectrum where the luminescence intensity is low, and small levels of contamination may have a large effect on the measured polarization.

- Figures 5.37 through $\mathbf{5 . 4 5}$ show luminescence circular polarization measured as 


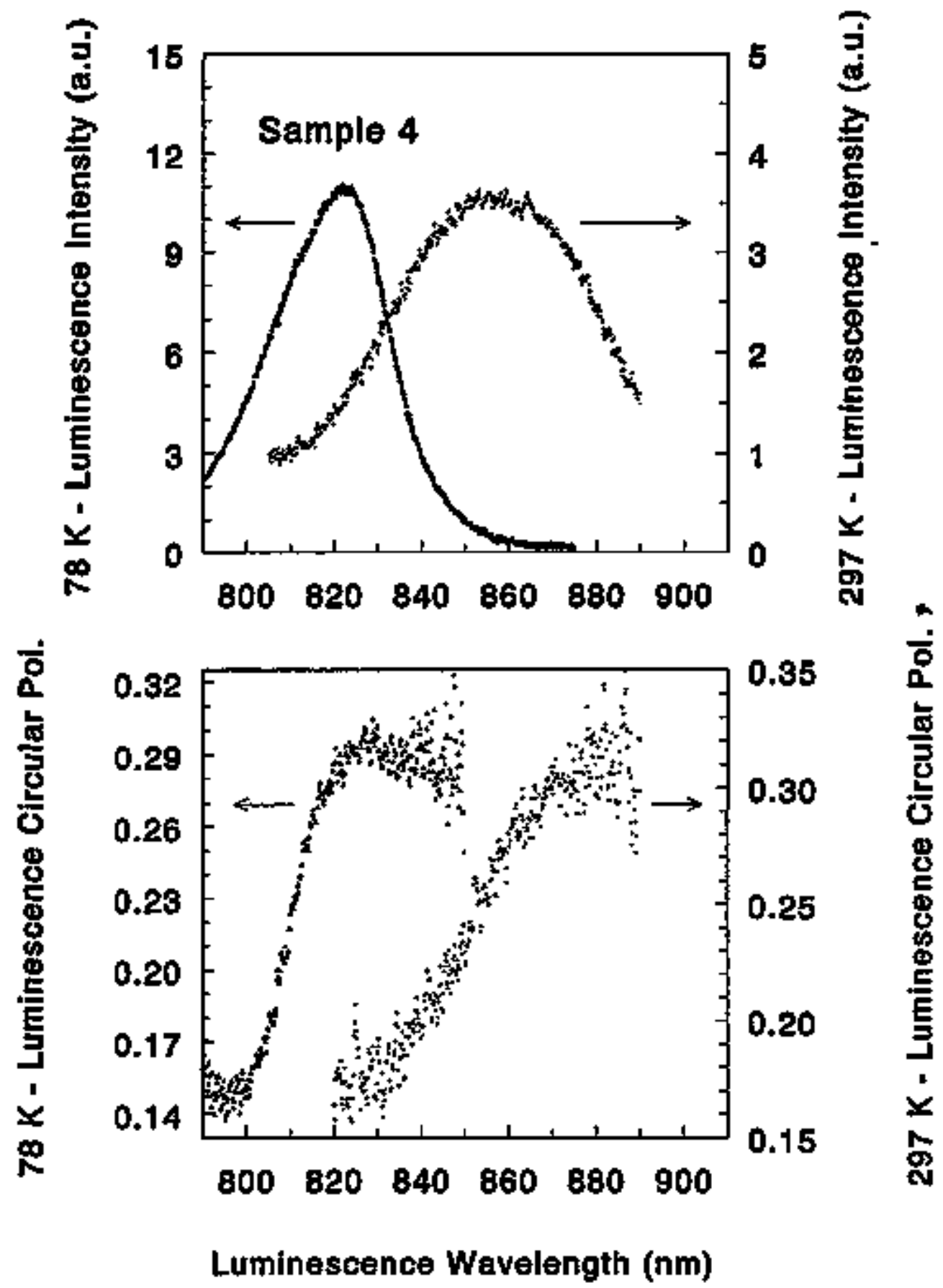

Figure 5.36: Luminescence spectrum and corresponding circular polarization of luminescence for sample \#4 at $78 \mathrm{~K}$ and $297 \mathrm{~K}$. Pump wavelength $=750 \mathrm{~nm}(78$ K), $780 \operatorname{nm}(297 \mathrm{~K})$. 
a function of pump wavelength. These measurements were performed in the same manner as the room temperature measurements. It necessary to utilize nore than one luminescence wavelength for only two samples (\#6 and \#7) in obtaining data for all pump wavelengths. For each of these two samples, a scale factor for polarization at each luminescence wavelength was estimated from the lominescence wavelength dependent circular polarization curve.

The data shown in Figures 5.37 through 5.45 may be compared with the room temperature data from Figures 5.19 through 5.27 in order to investigate any possible change with temperature in the Iuminescence circular polarization magnitude for a given sample. Equation (3.31) gave the relationship for the luminescence circular polarization as $P_{\gamma}=-P_{e}-\left(h \nu_{\xi_{k}}\right) P_{k}\left(h \nu_{\mathrm{lvm}}\right) R$. Room temperature and 78 $\mathrm{K}$ luminescence circular polarization data are compared at pump wavelengths (energies) corresponding to the lower polarization plateau ( $P_{0^{-}}^{\text {piateau }}$ ) and luminescence wavelengths corresponding to the maximum recombination radiation coupling facfor $\left(P_{h}=P_{h}^{\max }\right)$ for the particular sample and temperature. In this way, the room temperature and $78 \mathrm{~K}$ polarization values being compared may be expressed as

$$
P_{\gamma}(T)=P^{\text {plateau }} P_{h}^{\max }(T) R(T)
$$

The electron polarization in the plateau region is assumed to be approximately $50 \%$ and independent of temperature, while $P_{h}^{\text {max }}$ and $R$ may depend on temperature. The $78 \mathrm{~K}$ data was recorded norninally at the luminescence waveleagth where $P_{h}^{\text {max }}$ occurred for each of the strained layer samples. The room temperature data for some samples, on the other hand, was recorded at luminescence wavelengths corresponding to a recombination radiation coupling factor less than the maxi- 


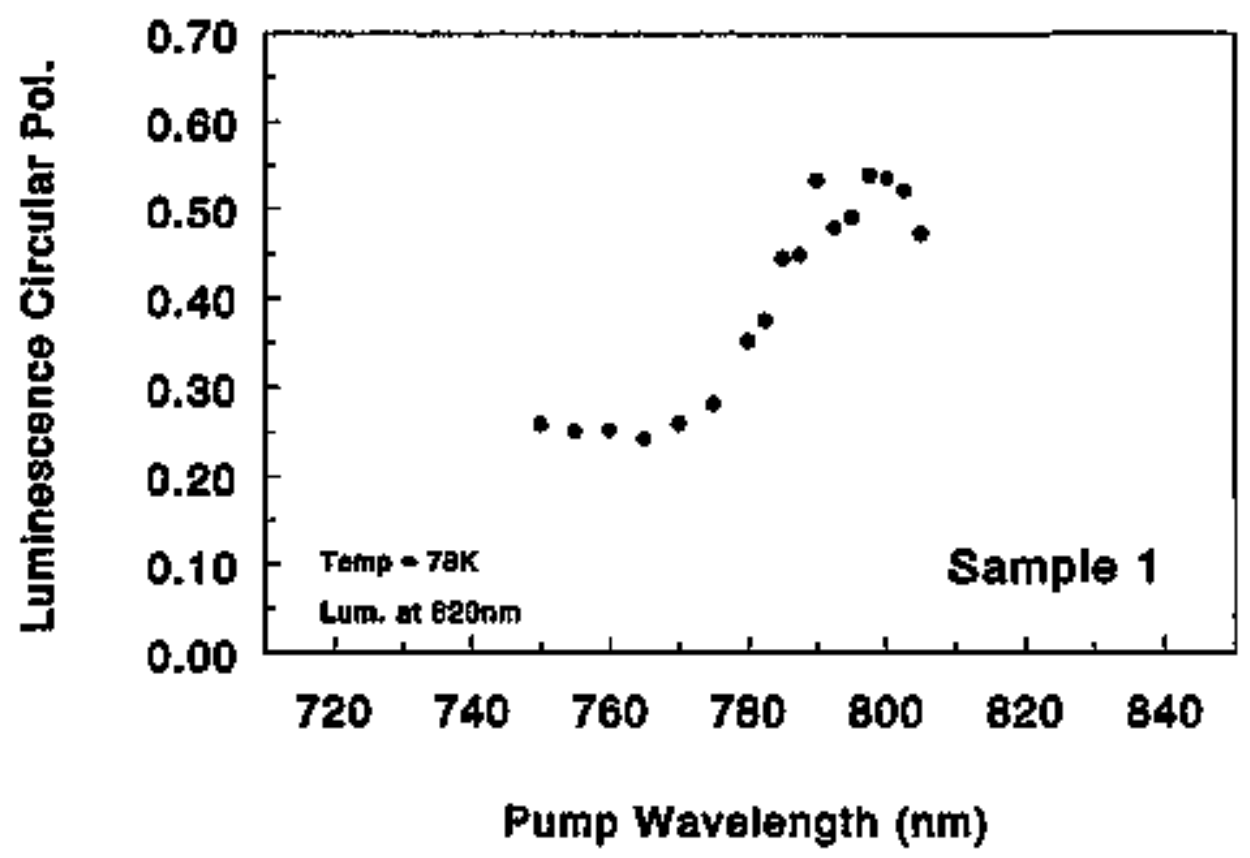

Figure 5.37: Pump wavelength dependence of luminescence circular polarization for sample \#1 at $78 \mathrm{~K}$. 


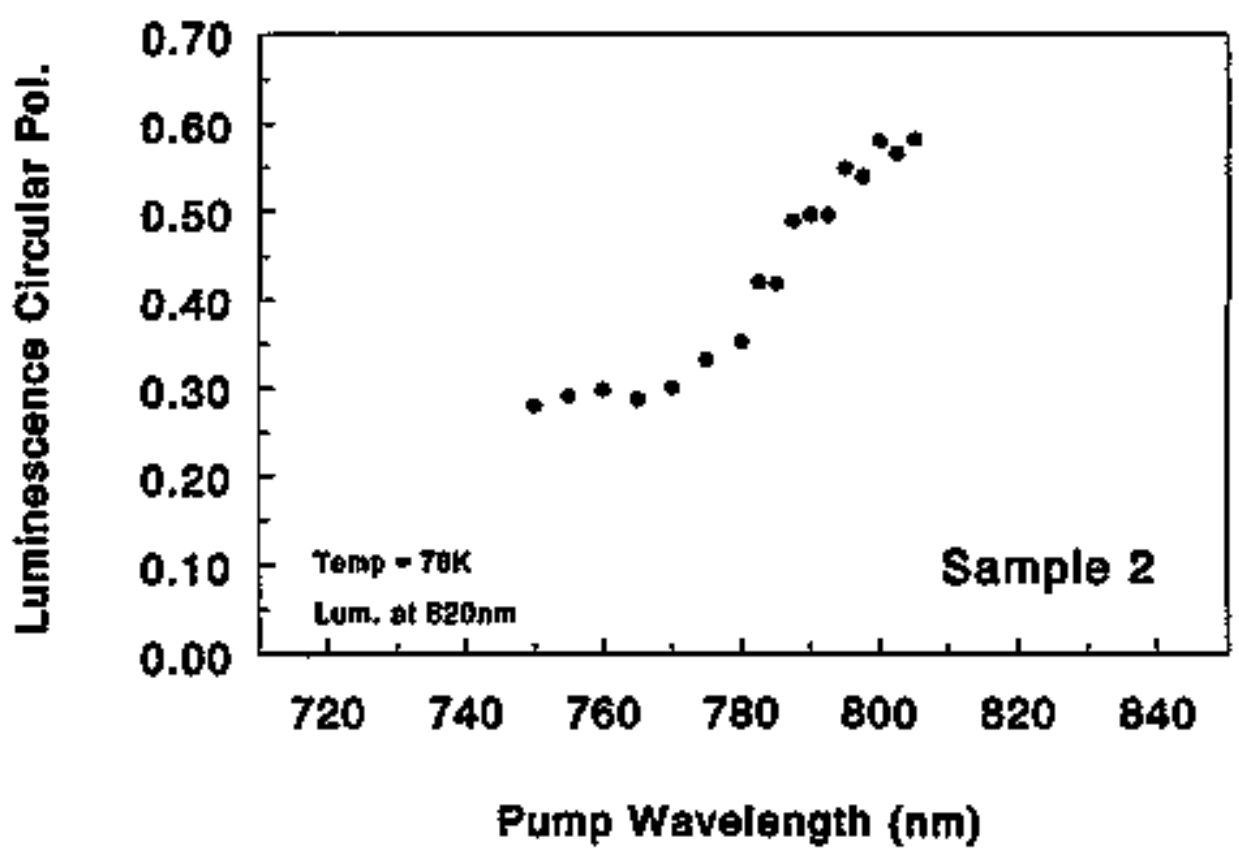

Figure 5.38: Pump wavelength dependence of luminescence circular polarization for sample \#2 at $78 \mathrm{~K}$. 


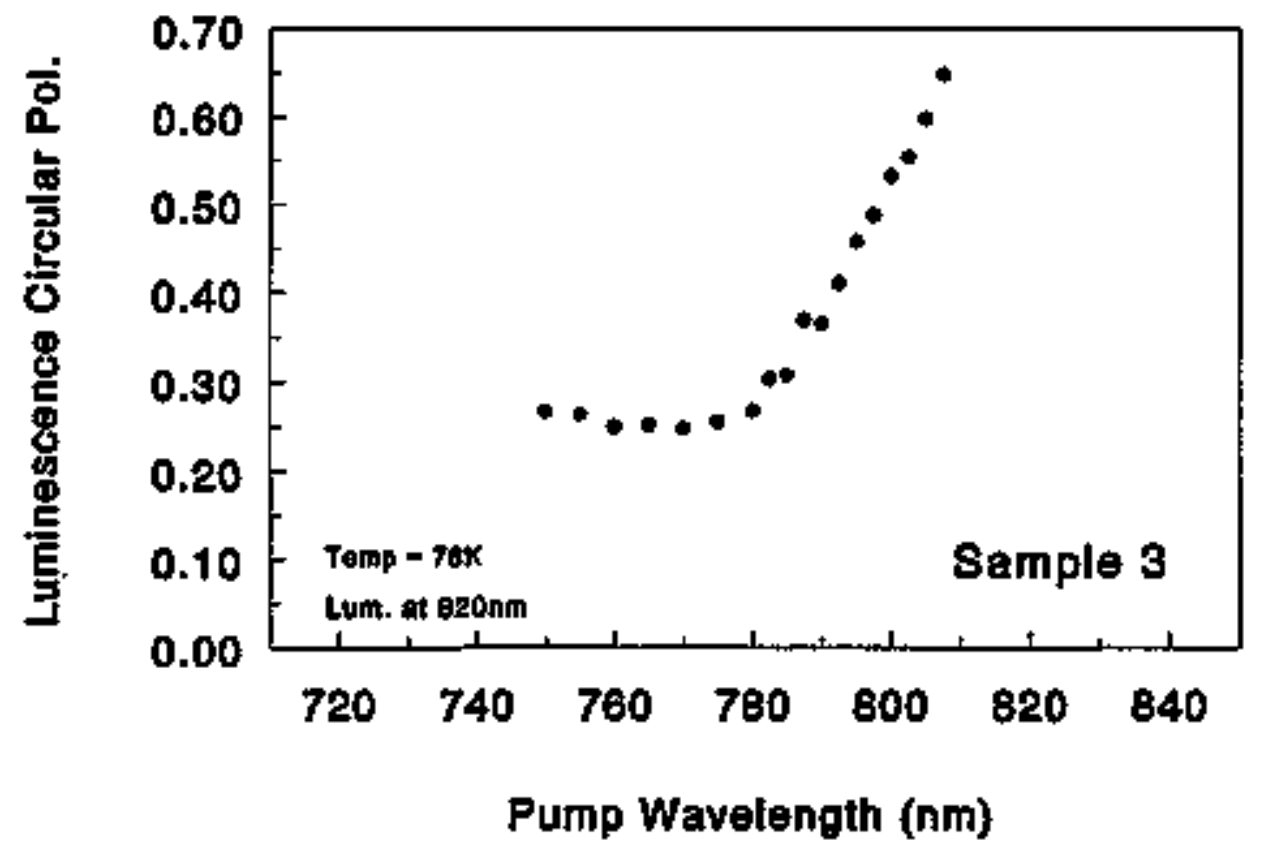

Figure 5.39: Pump wavelength dependence of luminescence circular polarization for sample \#3 at $78 \mathrm{~K}$. 


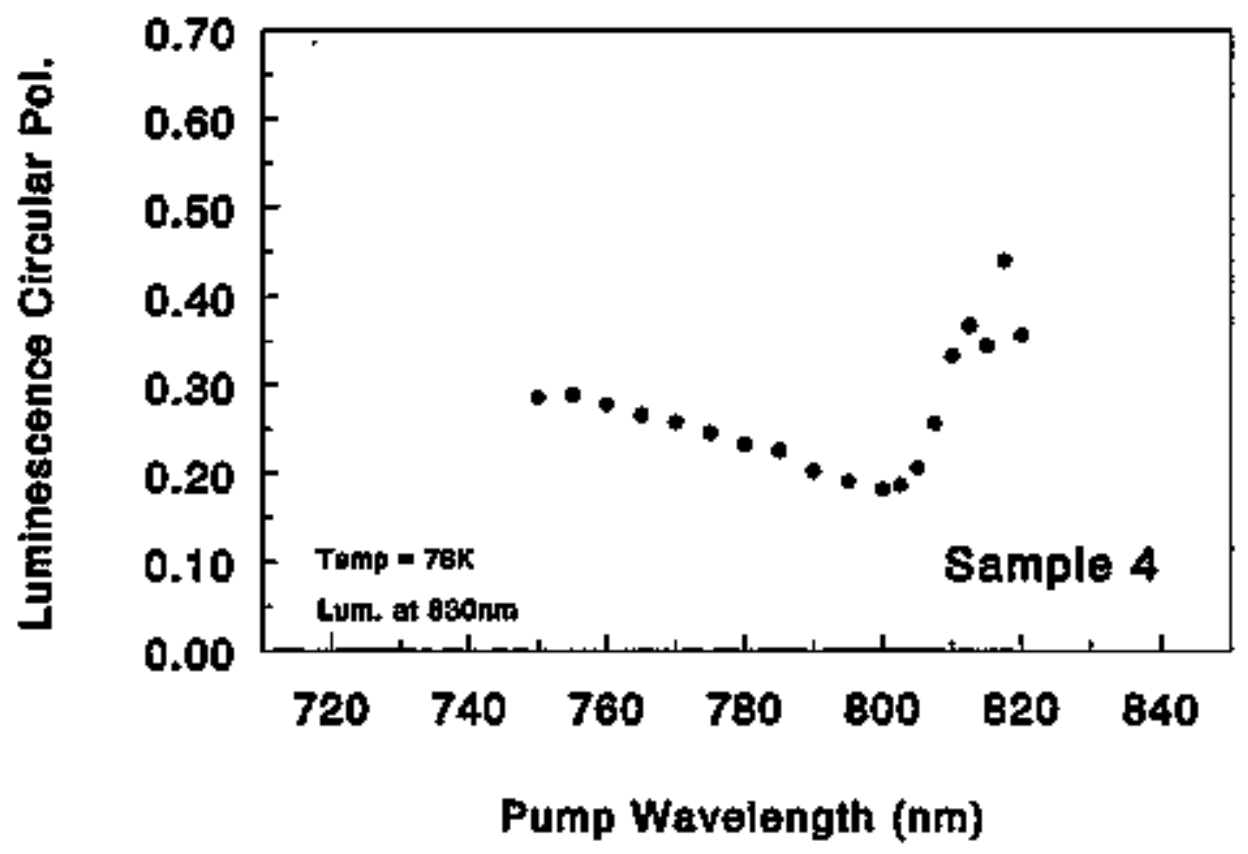

Figure 5.40: Pump wavelength dependence of luminescence circular polarization for sample \#4 at $78 \mathrm{~K}$. 


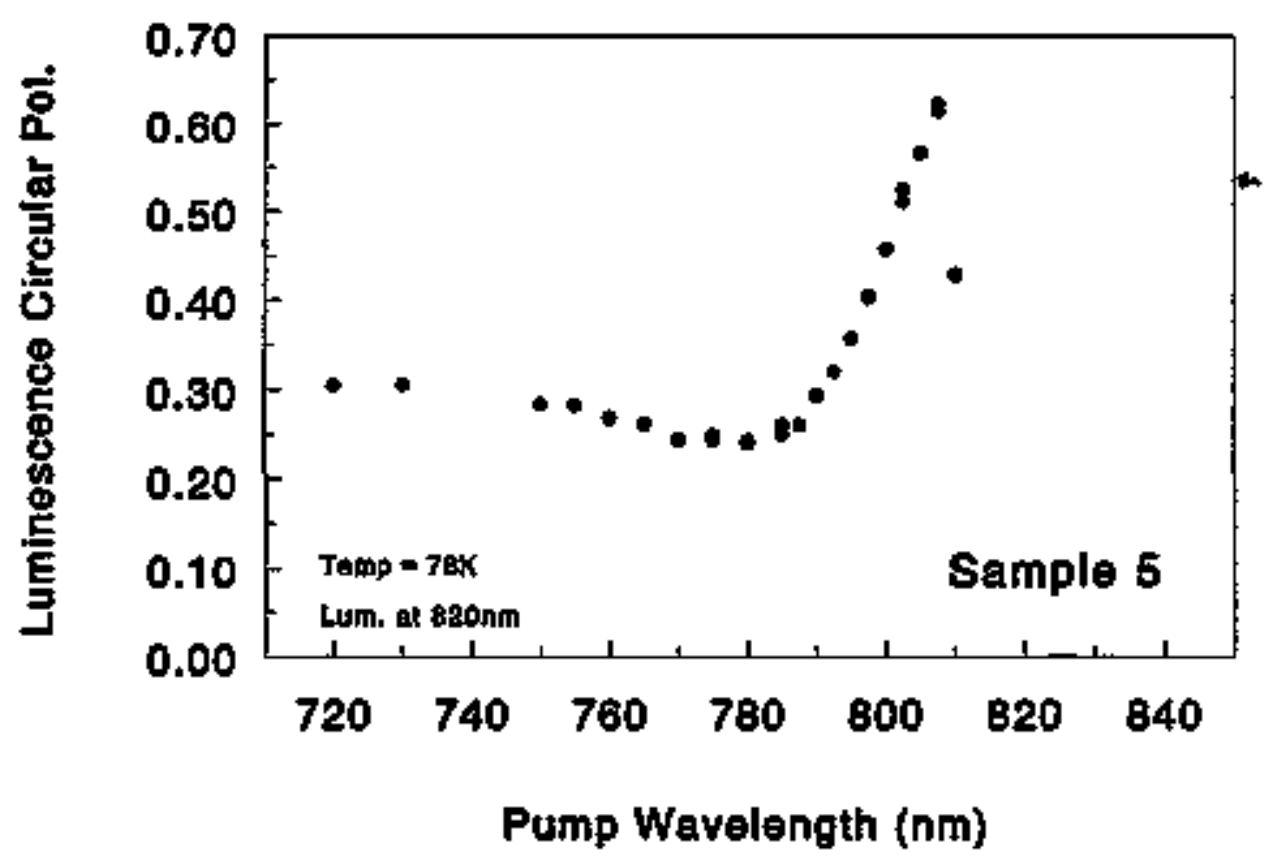

Figure 5.41: Pump wavelength dependence of luminescence circular polarization for sample $\# 5$ at $78 \mathrm{~K}$. 


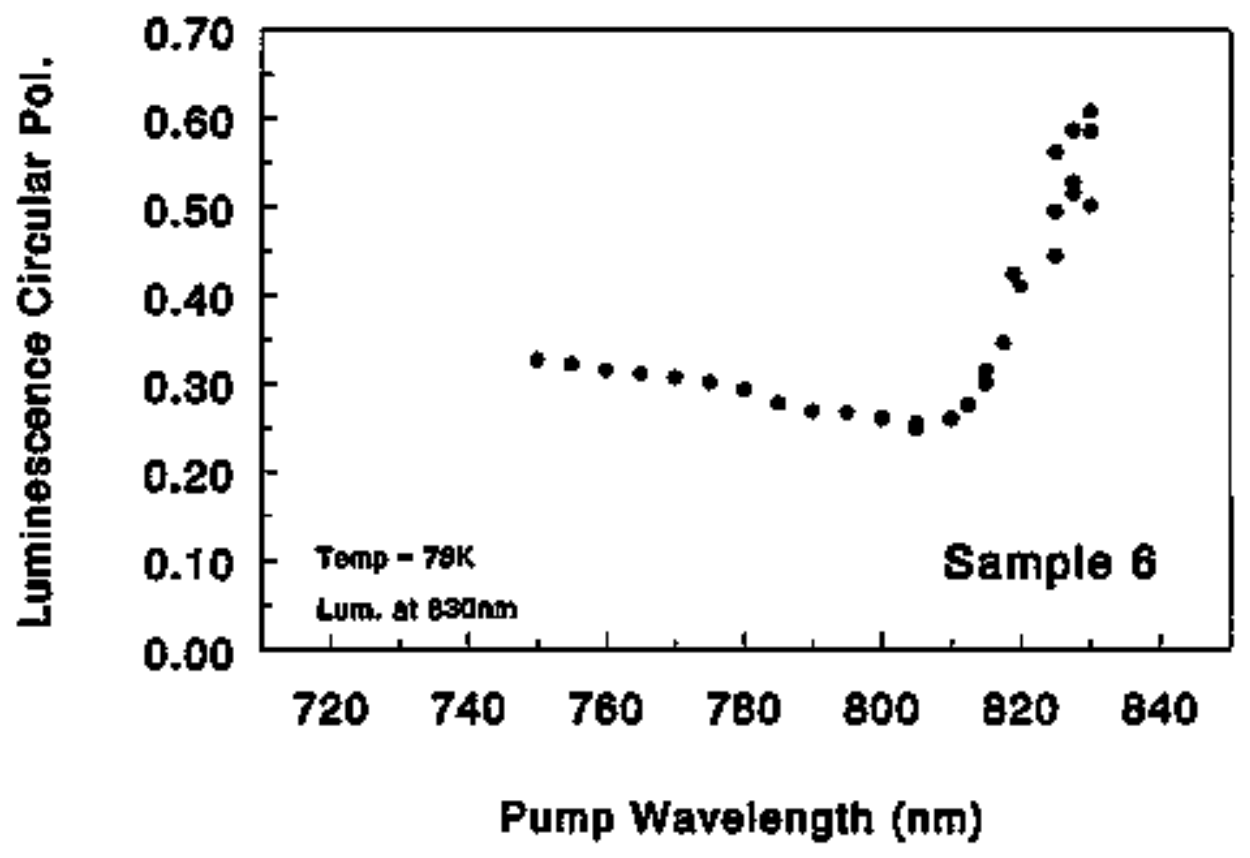

Figure 5.42: Pump wavelength dependence of luminescence circular polarization for sample $\# 6$ at $78 \mathrm{~K}$. 


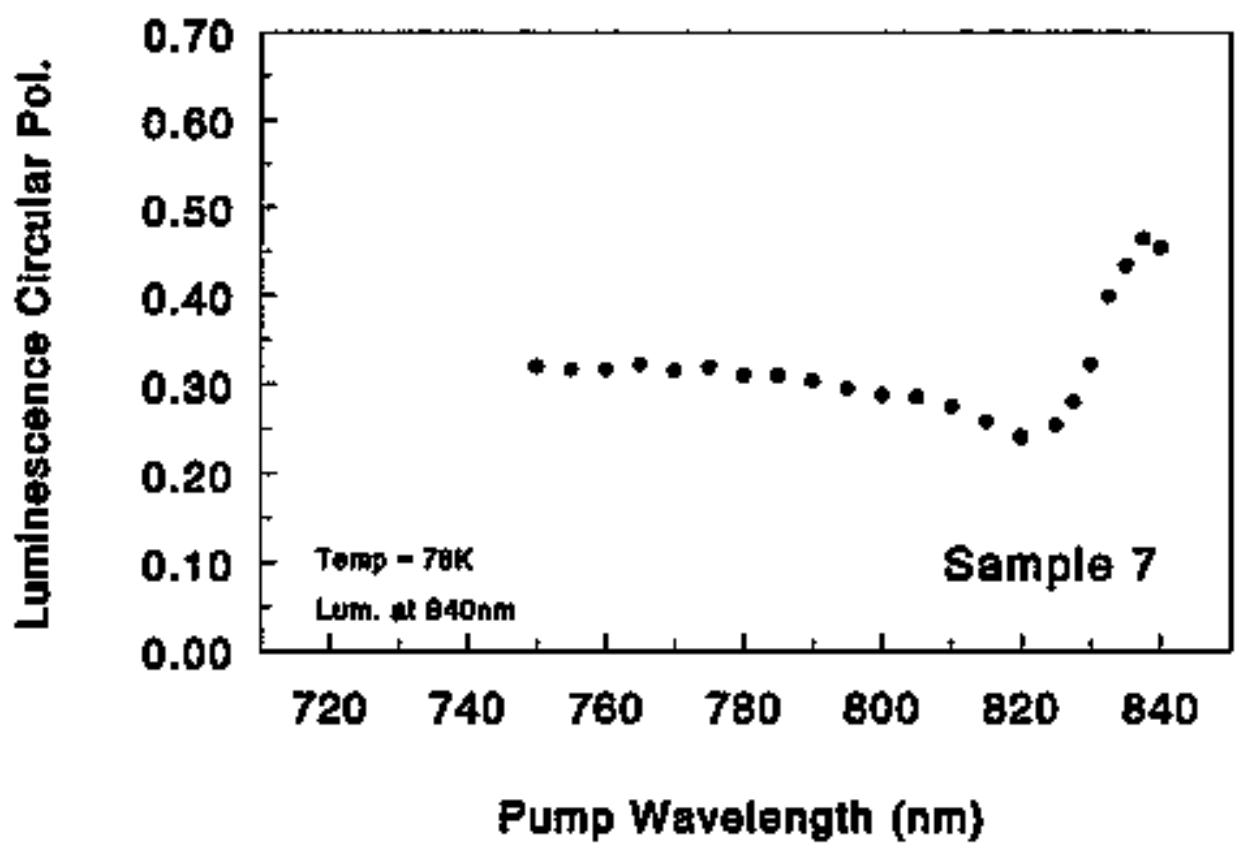

Figare 5.43: Pump wavelength dependence of luminescence circular polarization for sample \#7 at $78 \mathrm{~K}$. 


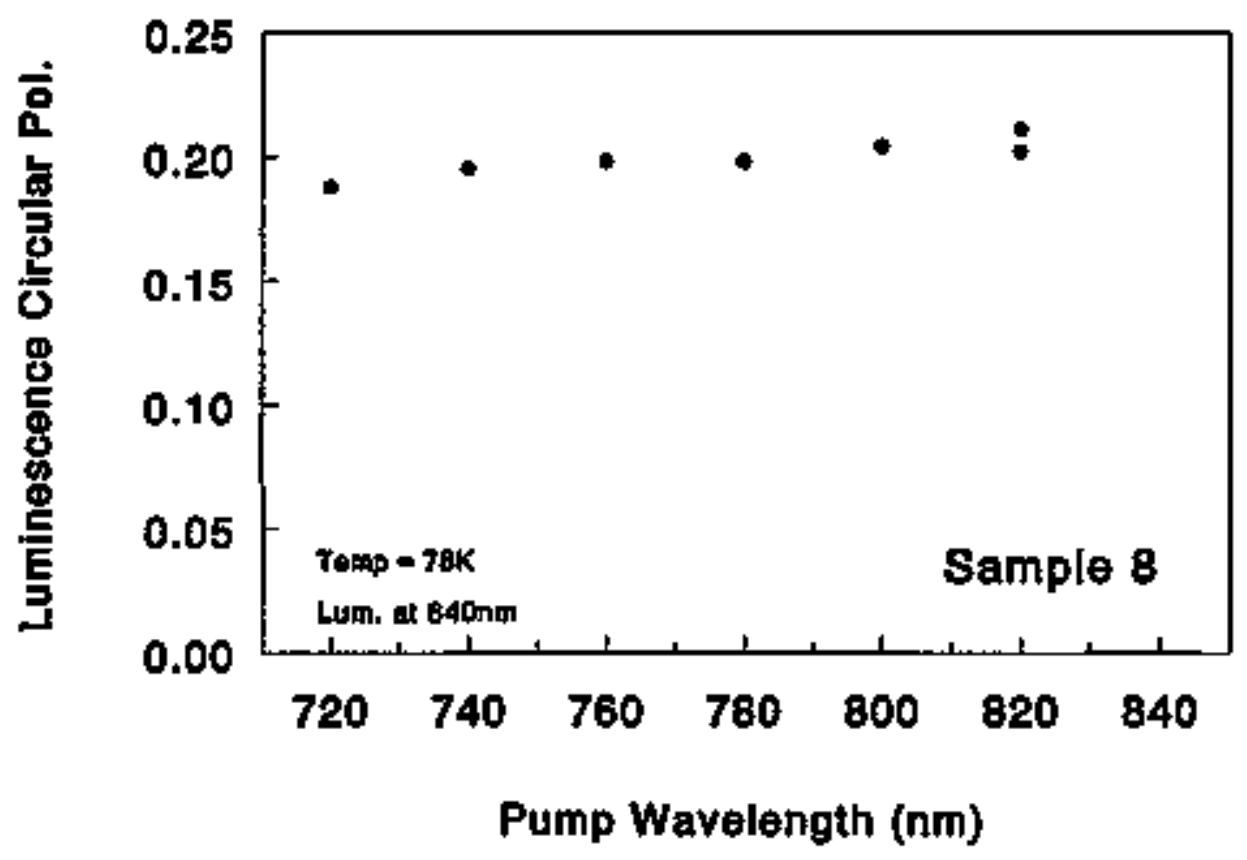

Figure 5.44: Pump wavelength dependence of luminescence circular polarization for tmple \#8 at $78 \mathrm{~K}$. 


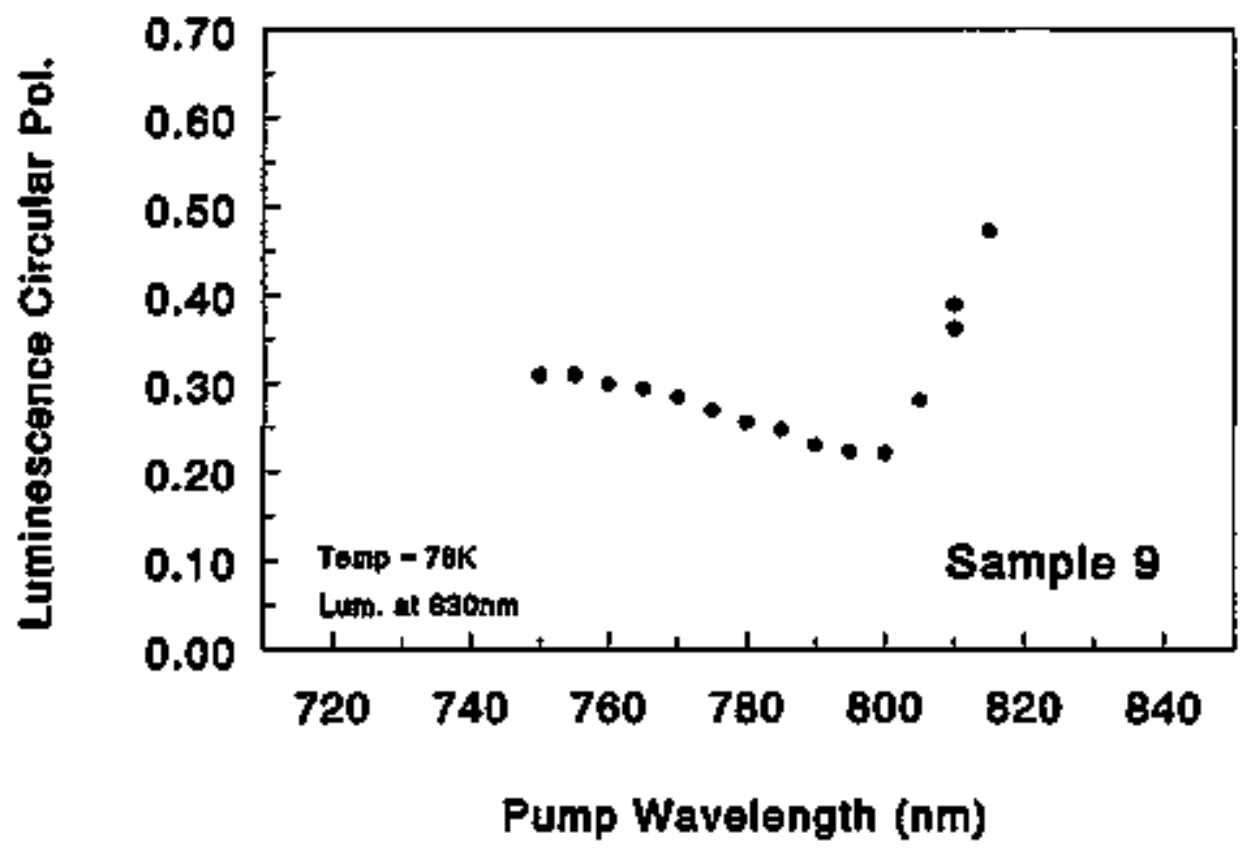

Figure 5.45: Pump wavelength dependence of luminescence circular polarization for sample \#9 at $78 \mathrm{~K}$. 
mum observed value. This is because the luminescence wavelength corresponding to the maximum recombination radiation coupling factor was in a region of the spectrum where the intersity was too low for practical use. For those cases, the polarization data is scaled by an appropriate factor which is obtained from the sample's luminescence wavelength dependent polarization curve. For example, the room temperature luminescence circular polarization data for sample \#6 shown in Figure 5.24 is representative of measurements taken at a luminescence wavelength of $840 \mathrm{~nm}$ while maximum polarization occurs at $\approx 890 \mathrm{~nm}$. This data must therefore be scaled by a factor of $P_{\mathrm{r}}\left(\lambda_{\mathrm{l} \mathrm{m}}=890 \mathrm{~nm}\right) / P_{\mathrm{\gamma}}\left(\lambda_{\mathrm{T} \mathrm{rm}}=840 \mathrm{~nm}\right)$, where the values of $P_{\gamma}$ are obtained from the huminescence circular polarization curve given in Figure 5.14.

Table 5.2 lists $78 \mathrm{~K}$ and room temperature luminescence circular polarization values for comparison. Values are representative of the indicated pump wavelength and the maximum recombination radiation coupling factor for the given sample and temperature. For each of the strained samples, the $78 \mathrm{~K}$ polarization value chosen for the table was the largest recorded value in the plateau region. The room temperature values were then chosen to correspond to a pump wavelength $35-40 \mathrm{~nm}$ longer than the pump wavelength of the $78 \mathrm{~K}$ data used. The 35$40 \mathrm{~nm}$ wavelength difference accounts for the temperature dependent band gap shrinkage. Tables 4.1 and 5.1 may be referred to for nominal sample parameters and $\mathrm{X}$-ray measured lattice parameters, respectively. Table 5.2 shows that the nonstrained GaAs sample (\#8) exhibits virtually no change in luminescence circular polarization between the two temperatures. Six of the eight strained layer samples 


\begin{tabular}{ccccc} 
& $297 \mathrm{~K}$ & $297 \mathrm{~K}$ & $78 \mathrm{~K}$ & $78 \mathrm{~K}$ \\
Sample \# & Pump $\lambda(\mathrm{mm})$ & $P_{r}$ & Pump $\lambda(\mathrm{nm})$ & $P_{\mathrm{r}}$ \\
\hline 1 & 785 & .292 & 750 & .259 \\
2 & 800 & .305 & 760 & .298 \\
3 & 790 & .319 & 750 & .267 \\
4 & 790 & .316 & 755 & .289 \\
5 & 770 & .315 & 730 & .306 \\
6 & 790 & .314 & 750 & .327 \\
7 & 805 & .279 & 765 & .322 \\
8 & 835 & .205 & 800 & .204 \\
9 & 790 & .338 & 750 & .309 \\
\hline
\end{tabular}

Table 5.2: Comparison of room temperature circular polarization with that at $78 \mathrm{~K}$. Values are representative of pump wavelengths within the $P_{\mathrm{e}^{-}} \approx 50 \%$ polarization plateau and luminescence wavelengths corresponding to the maximum recombination radiation coupling factor.

also exhibit either no change or a small decrease in polarization when the sample is cooled to $78 \mathrm{~K}$. However, the two strained layer samples with the lowest level of lattice strain (\#6 and \#7) show an increase in polarization upon cooling to $78 \mathrm{~K}$. These results are consistent with a situation where, upon cooling to $78 \mathrm{~K}$, there is little or no change in the spin relaxation factor $R$, but there is an increase in the maximum recombination radiation coupling factor $P_{h}^{\max }$ for those samples which had $P_{h}^{\max }<1$ at room temperature.

Several of the $78 \mathrm{~K}$ plots from Figures $5.37-5.45$ show a region where the luminescence circular polarization actually decreases, wjth increasing pump wave- 
length, before ultimately exhibiting the characteristic enhancement due to strain. This actually complicates the luminescence polarization comparison somewhat and may explain why some of the samples show a decrease in polarization as large as $20 \%$ (relative) upon cooling to $78 \mathrm{~K}$. These apparent decreases may not be real but related to the 'dip' and the fact that there was, unfortunately, no data recorded at pump wavelengths fally outside of the 'dip' region for some samples.

\subsubsection{Measurements at $12 \mathrm{~K}$}

In addition to $78 \mathrm{~K}$ measurements, the pump wavelength dependence of lumivescence circular polarization was measured at $\approx 12 \mathrm{~K}$ for several of the samples. The data is shown in Figures 5.46 through 5.51 and may be compared with room temperature data as was the $78 \mathrm{~K}$ data. Again, no significant change in the luminescence circular polarization relaxation factors is observed.

It is observed that the small 'dip' in polarization noticed in the $78 \mathrm{~K}$ data is more pronounced in the $12 \mathrm{~K}$ data. In fact, the 'dip' is so severe for samples \#6 and \#9 that the polarization decreases to almost zero with no overall polarization enhancement seen. We believe that this phenomenon seen in the $78 \mathrm{~K}$ and 12 $\mathrm{K}$ data may be explained by transitions involving the impurity levels becoming increasingly predominant at lower temperature. It was shown in Chapter $\mathbf{3}$ (eq. (3.3)) that the ratio of absorption probability to emission probability is given by $\frac{A}{A^{\prime}}\left(e^{h \nu / k_{B} T}-1\right)$ for a band to band transition. Transitions to or from acceptor irspurity states were not considered. When one calculates transition probabilities for the acceptor states it is found that the ratio of absorption to emission probability 


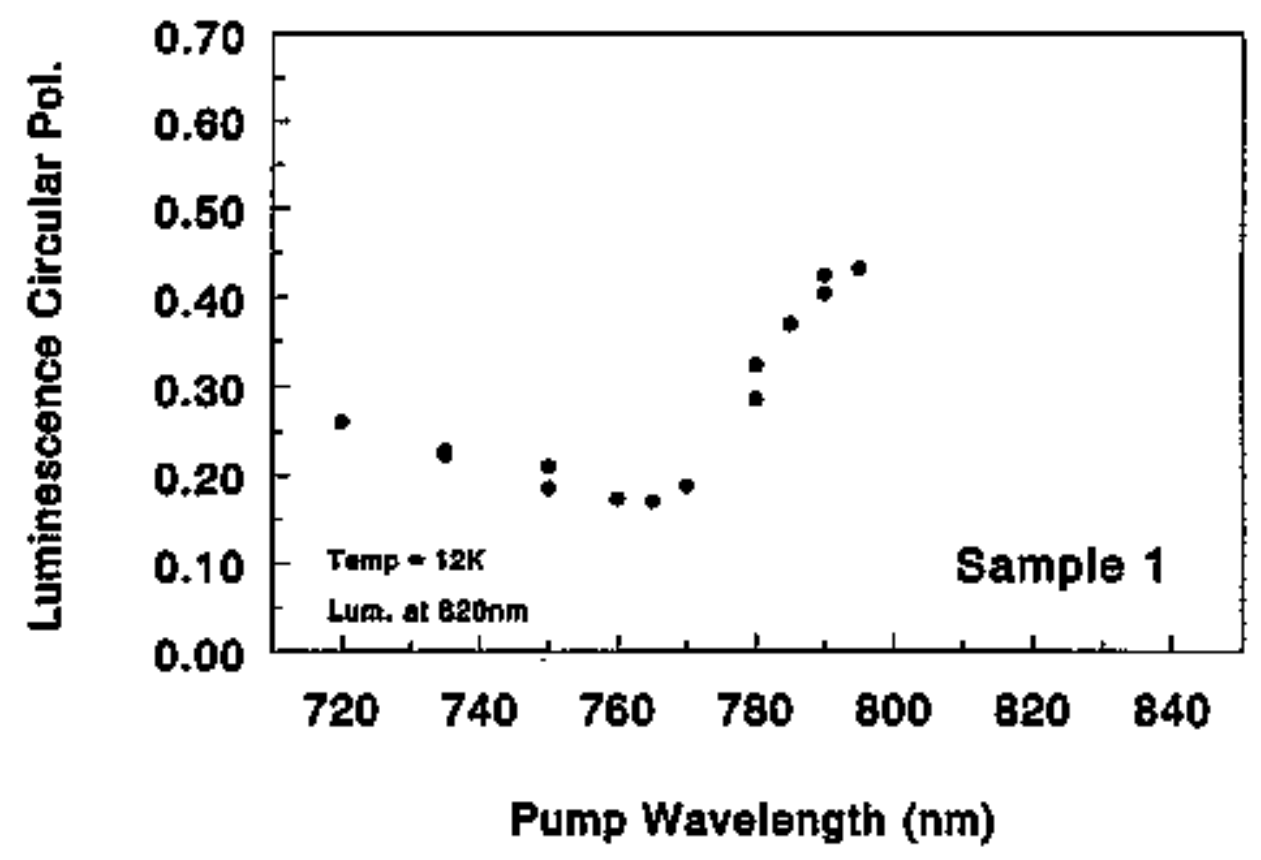

Figure 5.46: Pump wavelength dependence of luminescence circular polarization for sample \#1 at $\approx 12 \mathrm{~K}$. 


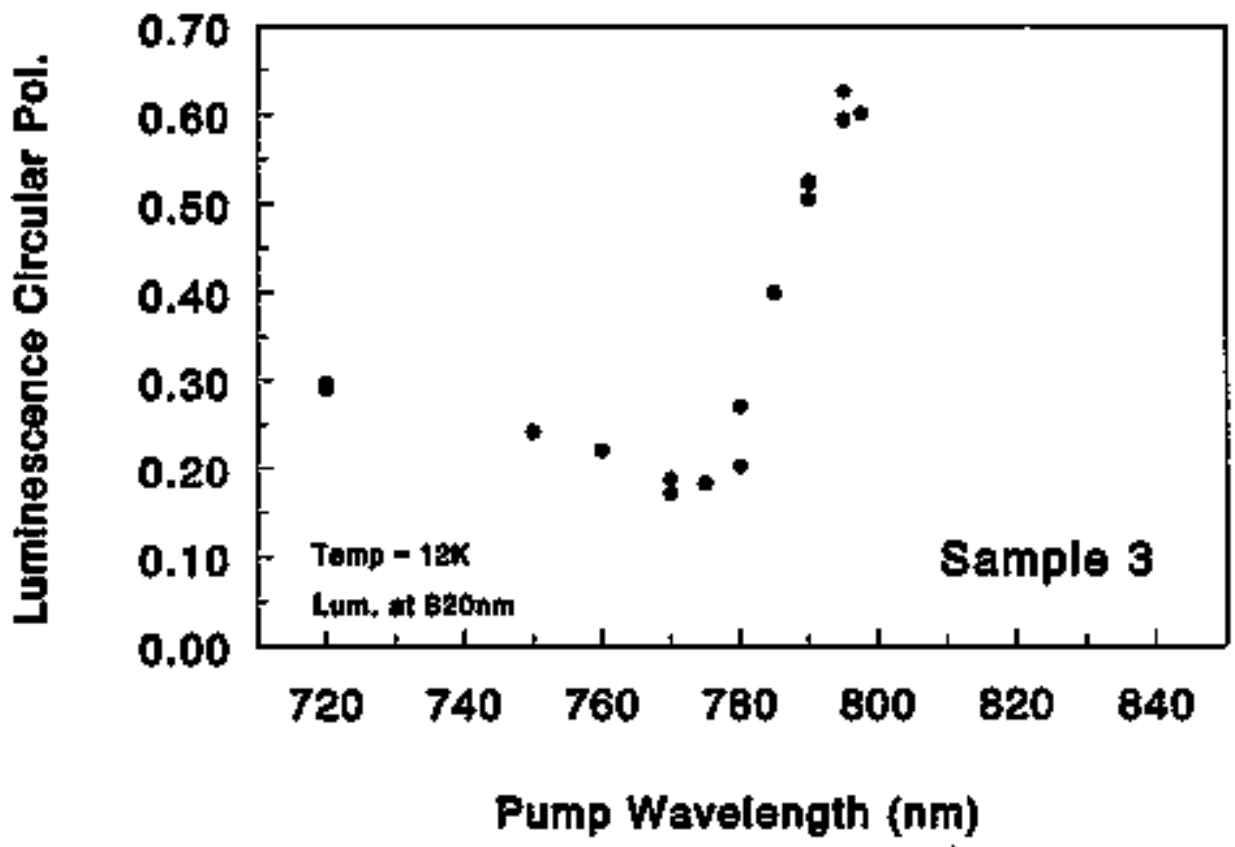

Figure 5.47: Pump wavelength dependence of luminescence circular polarization for sample \#3 at $\approx 12 \mathrm{~K}$. 


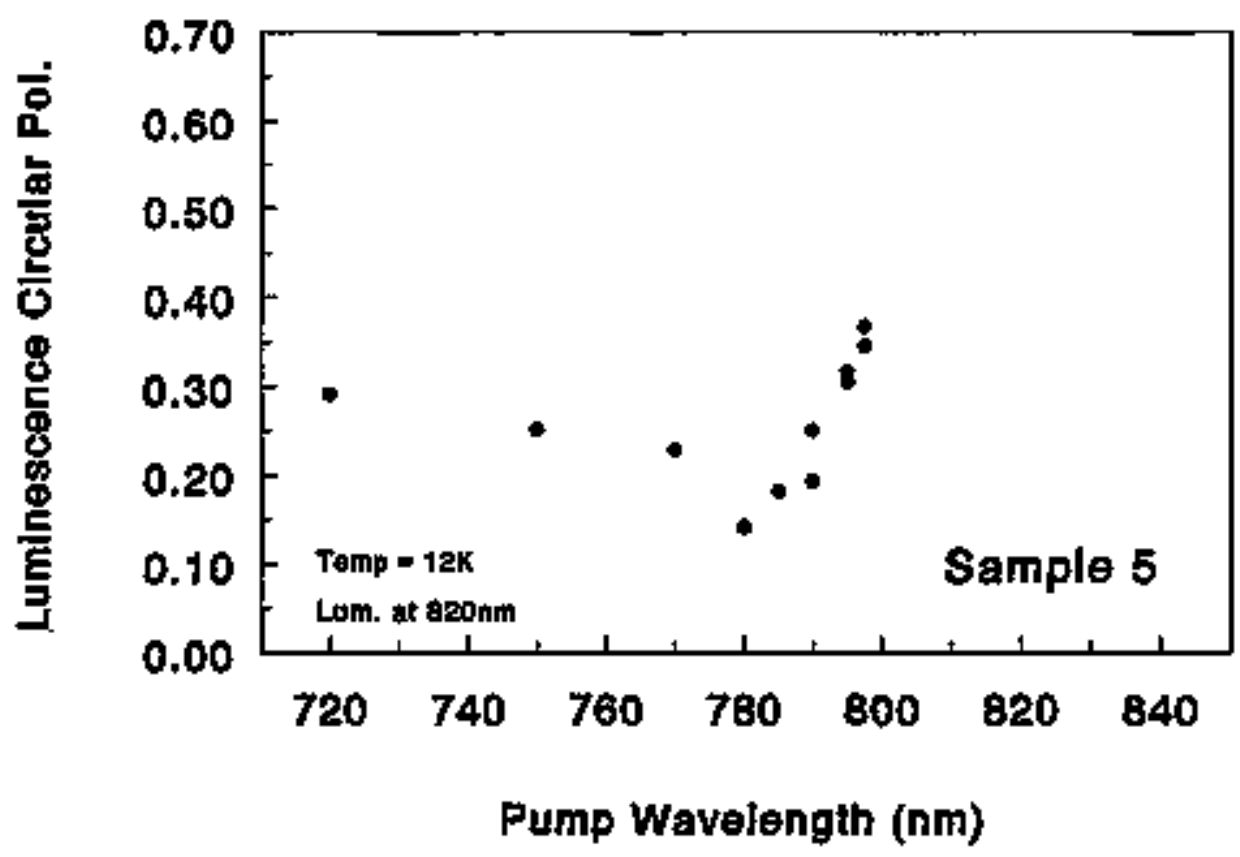

Figure 5.48: Pump wavelength dependence of luminescence circular polarization for sample \#5 at $\approx 12 \mathrm{~K}$. 


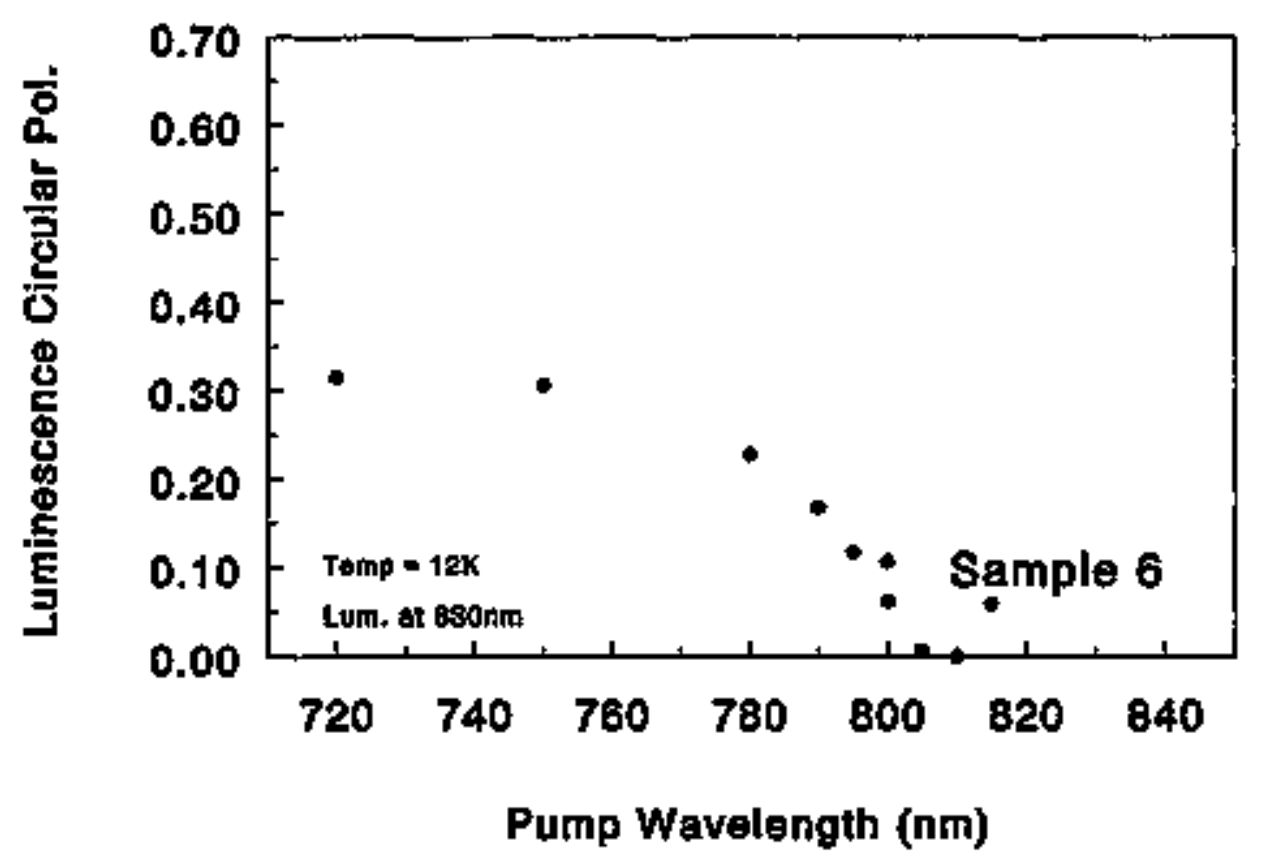

Figure 5.49: Pump waveleggth dependence of luminescence circular polarization for sample \#6 at $\approx 12 \mathrm{~K}$. 


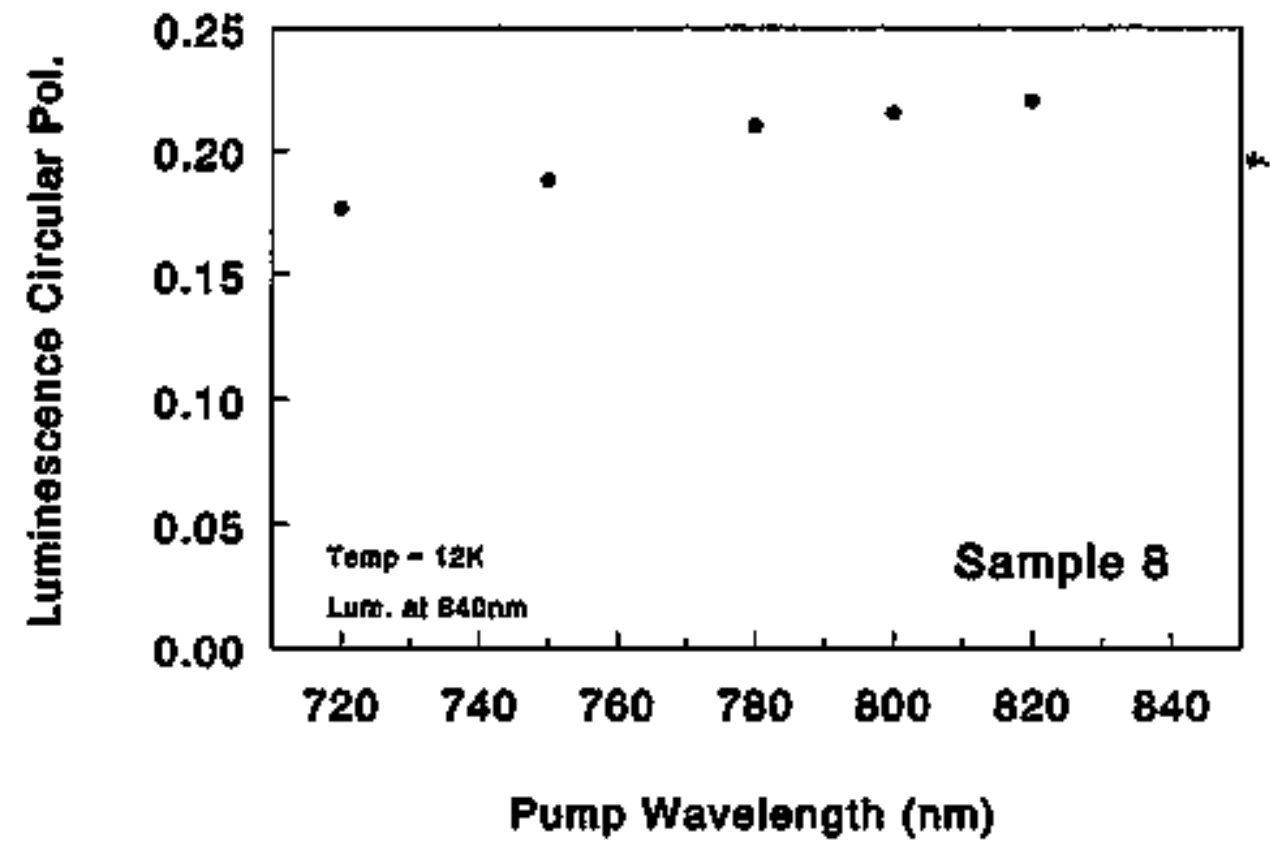

Figure 5.50: Pump wavelength dependence of luminescence circular polarization for sample \#8 at $\approx 12 \mathrm{~K}$. 


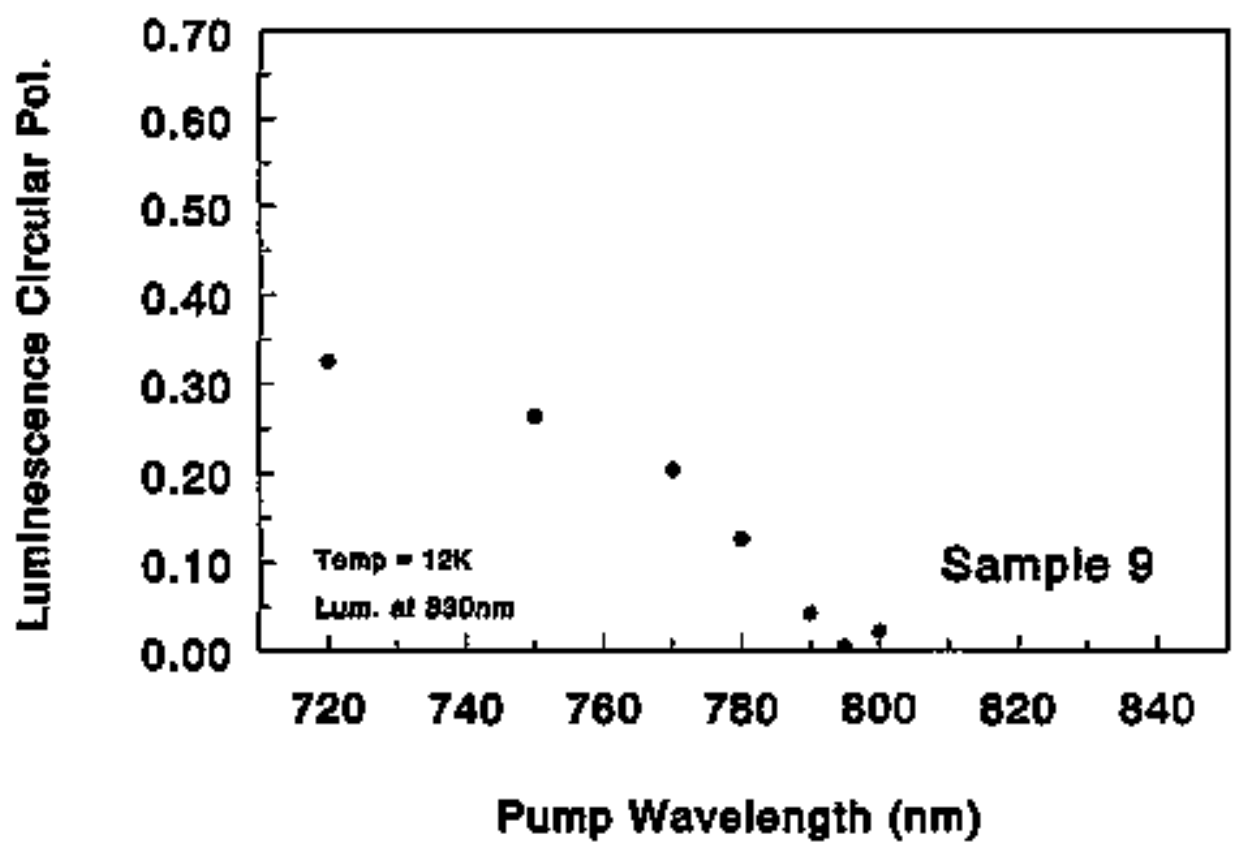

Figure 5.51: Purnp wavelength dependence of luminescence circular polarization for sample \#9 at $\approx 12 \mathrm{~K}$. 
is not given by the same relation, but ratber by

$$
\frac{\left(d P_{A(1 / 2)} / d E\right)_{\text {ebtorbb }}}{\left(d P_{A\{1 / 2)} / d E\right)_{\text {emit }}}=\frac{A}{A^{i}}\left[e^{h \nu / k_{E} T}\left(1+2 e^{\left(E_{j / 2}^{A}-E_{P}\right) / k_{B} T}+e^{\left(E_{1 / 2}^{A}-E_{F}\right) / k_{B} T}\right)-1\right]
$$

and

$$
\frac{\left(d P_{A(3 / 2)} / d E\right)_{\text {absorib }}}{\left(d P_{A(3 / 2)} / d E\right)_{\text {emit }}}=\frac{A}{A^{\prime}}\left[e^{h \nu / k_{B} T}\left(1+2 e^{\left(E_{1 / 2}^{A}-E_{F}\right) / k_{B} T}+e^{\left(E_{S / 2}^{A}-E_{F}\right) / k_{B} T}\right)-\dot{1}\right] .
$$

The acceptor states have the same symmetry as the valence states at $\Gamma$, and in the uniaxially strained crystal, the $J=3 / 2$ acceptor states are split under the lattice strain in the same manner as the valence states.[70] Equatjons (5.15) and (5.16) correspond to transitions involving the strain-split $m_{j}=1 / 2$ and $m_{j}=3 / 2$ acceptor states, respectively. These results differ from eq. (3.3) because the distribution function for the monovalent acceptor states differs from the standard Fermi-Dirac distribution function due to the intrinsic interdependence of the various acceptor states.[71]

Equations (5.15) and (5.16) indicate that for a specific photon energy, the spin polarization associated with excitation from the impurity levels is not necessarily equivalent to the coupling factor associated with recombination to the impurity levels. Furthermore, the difference is expected to be temperature dependent because of the presence of the temperature dependent $E_{F}$ in the equations. Qualitatively, the 'dip' observed in the low temperature circular polarization data can be explained as an increased level of transitions from the $m_{j}=1 / 2$ impurity states relative to transitions from the valence bands, while the recombination process does not exhibit such an increase of transitions involving the $m_{j}=1 / 2$ impurity states. 


\section{Chapter 6}

\section{Analysis and Conclusions}

The data presented in Chapter 5 provide much information regarding spin polarization and lominescence circular polarization in uniaxially strained GaAs. First, when luminescence circular polarization data from strajned layer samples were compared with electron spin polarization data (photo-emission), several samples showed that the maximum luminescence circular polarization corresponded to an electron spin polarization of $\approx 100 \%$. Also, for the six most highly strained samples, luminescence circular polarization data showed an approximate twofold enbancement in polazization from waveleagths in the lower polarization plateau to wavelengths where the polarization reached a maximum. Finally, room temperature data presented in Figures 5.19 through 5.27 showed that the recombination radiation coupling factor $P_{h}$ and electron spin polarization factor $P_{e-}$ are equivalent as expected and that the spin relaxation factor $R$ is independent of pump and luminescence photon energy. Otberwise, one would not expect equivalence (with an appropriate scale factor) of the pump/luminescence wavelength dependent polarizations.

In Chapter 3, an estimate was made of the effect of nonzero $\vec{k}$ on the spin polarization obtainable by optical pumping. The calculation was performed for 
photon energies measured relatjve to the bandgap and parameterized by the strain induced valence band splitting $\delta_{s}$ so that the results could be generalized to any degree of lattice strain. For transitions from the $m_{j}=3 / 2$ valence band, the expected spin polarization was only found to be reduced from $100 \%$ to $95 \%$ as the photon energy was increased from the $m_{j}=3 / 2$ gap minimum to the $m_{j}{ }^{*}=1 / 2$ gap minimum. With this calculation, the assumption that over at least some portion of the luminescence spectrum the respective $m_{j}=3 / 2$ and $m_{j}=1 / 2$ coupling factors are $-100 \%$ and $+100 \%$ appears to be a reasonable approximation. Unfortunately though, this calculation was made for parabolic bands with no doping-induced (or otherwise) band tailing. The samples used in the study have significant band tails due to the high level of p-type doping and this complicates any calculation involving the valence band wave functions near band edge. Still, the hypothesis of $\pm 100 \%$ coupling factors may be made and tested (for consistency) with the data.

Considering the observations regarding the luminesceece circular polarization data and the calculated estimate of the effect of non-zero $\vec{k}$ on spin polarization, we formulate the following model for electron spin polarization in the strained layer GaAs photocathodes.

- $P_{e^{-}}=P_{h}=-100 \%$ and $+100 \%$ for transitions involving the $\left|\frac{3}{2}, \frac{3}{2}\right\rangle$ and $\left|\frac{3}{2}, \frac{1}{2}\right\rangle$ valence bards, respectively. This is assumed valid for photon energies within the observed luminescence spectrum.

- The spin relaxation factor $R$ for a given sample is independent of photon energy.

- For those samples with relatjvely low kevels of lattice strain, maximum lumi- 
nescence circular polarization values (at the longest pump/lum. wavelengths) less than $R \times 100 \%$ are the result of incomplete suppression of transitions to/from the $m_{j}=1 / 2$ valence band. This is a direct result of the band tailing present in the samples.

- For increasing luminescence/pump photon energies, the circular polarization level moves toward a value corresponding to $P_{\mathrm{t}^{-}}=P_{h}=50 \%$ for two distinct reasons. First, with increasing pump/luminescence photon energy, there is an increasing level of transitions involving the $\left\langle\frac{3}{2}, \frac{1}{2}\right\rangle$ band relative to the $\left\langle\frac{3}{2}, \frac{3}{2}\right\rangle$ valence band. The trassition ratio tends toward 1:3 as determined solely by by the Glebsch-Gordon coefficients involved in the dipole transitions. Second, $P_{e}-$ and $P_{h}$ must, for sufficiently large photon energy, tend towards $50 \%$ for each of the valence bands because the strain induced effects on band structure are no longer dominant over the $\vec{k}$ effects. The former reason is assumed to be dominant in the luminescence spectrum energy range.

Working within the framework of this model, the luminescence and X-ray data presented in Chapter 5 may be further analyzed. Analysis includes deconvolution of the luminescence contributions from the two $J=3 / 2$ valence bands and subsequent determination of the deformation potentials $a$ and $b$ for GaAs. A value of the deformation potential $d$ may also be determined with linearly polarized luminescence asymmetry measurements, as described in Appendix C. Finally, spin relaxation factors $R$ are determined for each sample and compared for the purpose of identifying the nature of the spin relaxation in the lumimescence data. 


\subsection{Deconvolution of Luminescence Spectra}

The expression for luminescence circular polarization from a strajned layer GaAs sample gaven in eq. (3.31). It becomes simplified under the condition of a fixed purnp photon energy $h v_{0}$ as

$$
P_{\gamma}\left(h \nu_{h u m}\right)=S P_{h}\left(h \nu_{l v m}\right)
$$

where $S$ is a constant equal to the product $P_{e}-\left(h \nu_{o}\right) R . \quad P_{h}$ in eq. (6.1) is the average of the contributing recombination radiation coupling factors for each of the $J=3 / 2$ valence bands. By assigaing fixed values of +1 and -1 to the individual coupling factors associated with the $m_{j}=3 / 2$ and $m_{j}=1 / 2$ valence bands, the averaged coupling factor is expressed as

$$
P_{h}\left(h \nu_{l s m}\right)=\frac{(+1) I^{(1)}\left(h \nu_{l u m}\right)+(-1) I^{(2)}\left(h \nu_{l u m}\right)}{I^{(1)}\left(h \nu_{l s m}\right)+I^{(2)}\left(h \nu_{l u m}\right)} .
$$

Here, $I^{(1)}$ and $I^{(2)}$ are the luminescence intensitjes related to the $\left\langle\frac{3}{2}, \frac{1}{2}\right\rangle$ and $\left|\frac{3}{2}, \frac{1}{2}\right\rangle$ valence bands, respectively. It is important to note that the $+1 / \mu 1$ assignments take into account the sign convention in eq. (5.12) chosen for plotting purposes. The luminescence spectrum may also be expressed in terms of the intensities associated with each valence band.

$$
I_{t u m}\left(h \nu_{l u m}\right)=I^{(1)}\left(h \nu_{l u m}\right)+I^{(2)}\left(h \nu_{l \mathrm{um}}\right)
$$

Equations (6.1) through (6.3) can be combined to form equations for the bandspecific intensities $I^{(1)}$ and $I^{(2)}$. Doing so, one obtajns

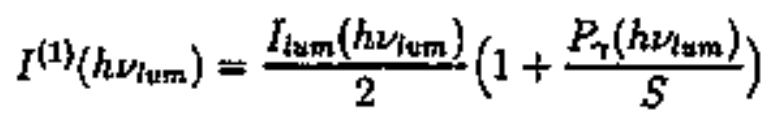




\begin{tabular}{ccc} 
Sample \# & $S$ & Comment \\
\hline 1 & $.265 \pm .005$ & Used $P_{\gamma}^{\max }$ \\
2 & $.270 \pm .005$ & Used $P_{\gamma}^{\max }$ \\
3 & $.290 \pm .005$ & Used $P_{\gamma}^{\max }$ \\
4 & $.315 \pm .005$ & Used $P_{\gamma}^{\operatorname{mam}}$ \\
5 & $.290 \pm .005$ & Used $P_{\gamma}^{\max }$ \\
6 & $.320 \pm .010$ & Used $2 \times P_{\gamma}(h \nu \sim 820 \mathrm{~nm})$ \\
7 & $.345 \pm .010$ & Used $2 \times P_{\gamma}(h \nu \sim 830 \mathrm{~nm})$ \\
\hline
\end{tabular}

Table 6.1: List of the choice of $S$ for various samples. $S$ is used in eqs. (6.4) and (6.5) for luminescence spectia deconvolution.

and

$$
I^{(2)}\left(h \nu_{l \mathrm{~km}}\right)=\frac{I_{\mathrm{ium}}\left(h \nu_{\mathrm{km}}\right)}{2}\left(1-\frac{P_{\gamma}\left(h \nu_{l u m}\right)}{S}\right) .
$$

These two equations may be used to deconvolve the room temperature lumidescence spectra obtained for the strained layer GaAs samples into separate spectra for transitions to each of the $J=3 / 2$ valence bands. The only parameter is the value of $S$ for a given sample's lumizescence circular polarization curve. For Iuminescence circular polarization curves which show a full twofold enhancement in polarization, $S$ is taken simply as the maximum polarization value reached. For curves which do not show a full twofold enhancement in polarization, $S$ is chosen as twice the polarization level observed in the platean region where $P_{h}$ is believed to correspond to approximately $50 \%$.

Room temperature lominescence spectra from the eight strained layer GaAs samples were deconvolved using eqs. (6.4) and (6.5). The spectra and correspond- 


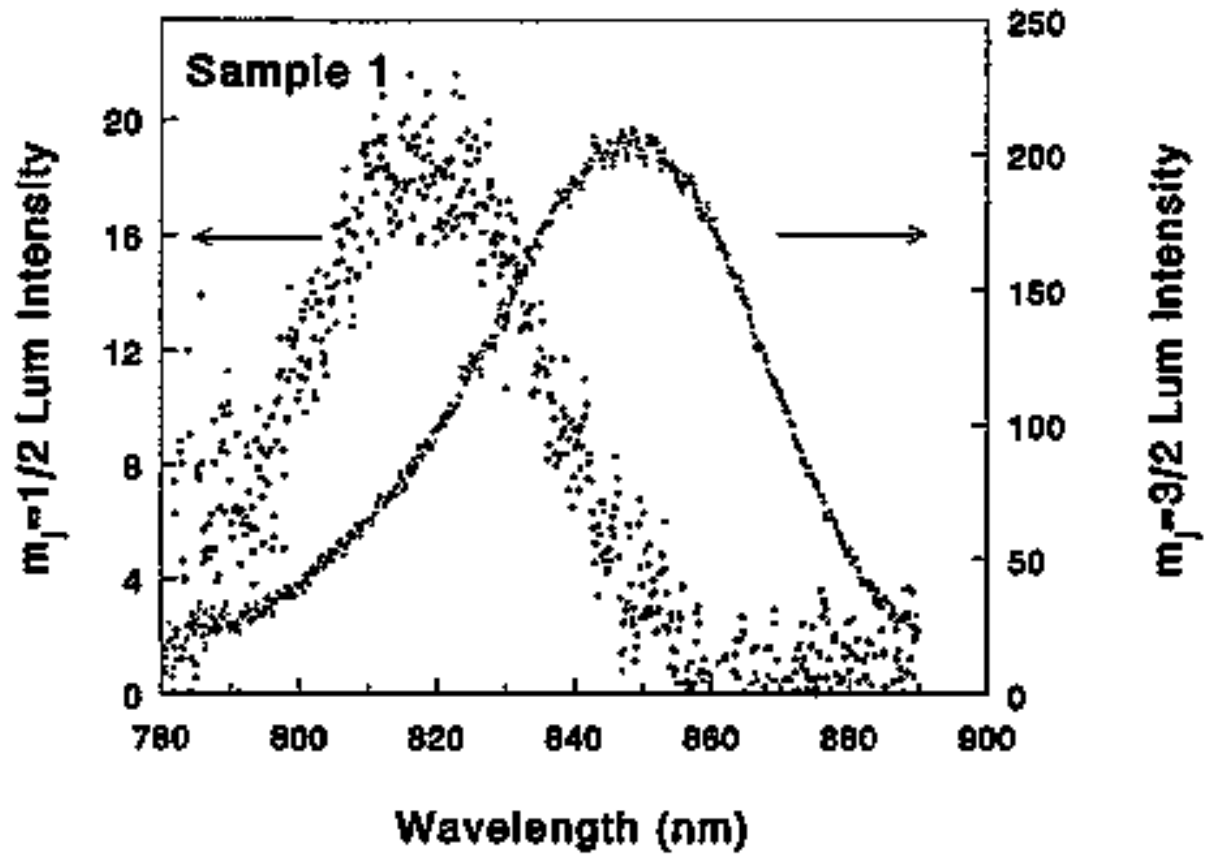

Figure 6.1: Deconvolved luminescence spectra from the two $J=3 / 2$ valence bands for sample \#1 at room temperature. 


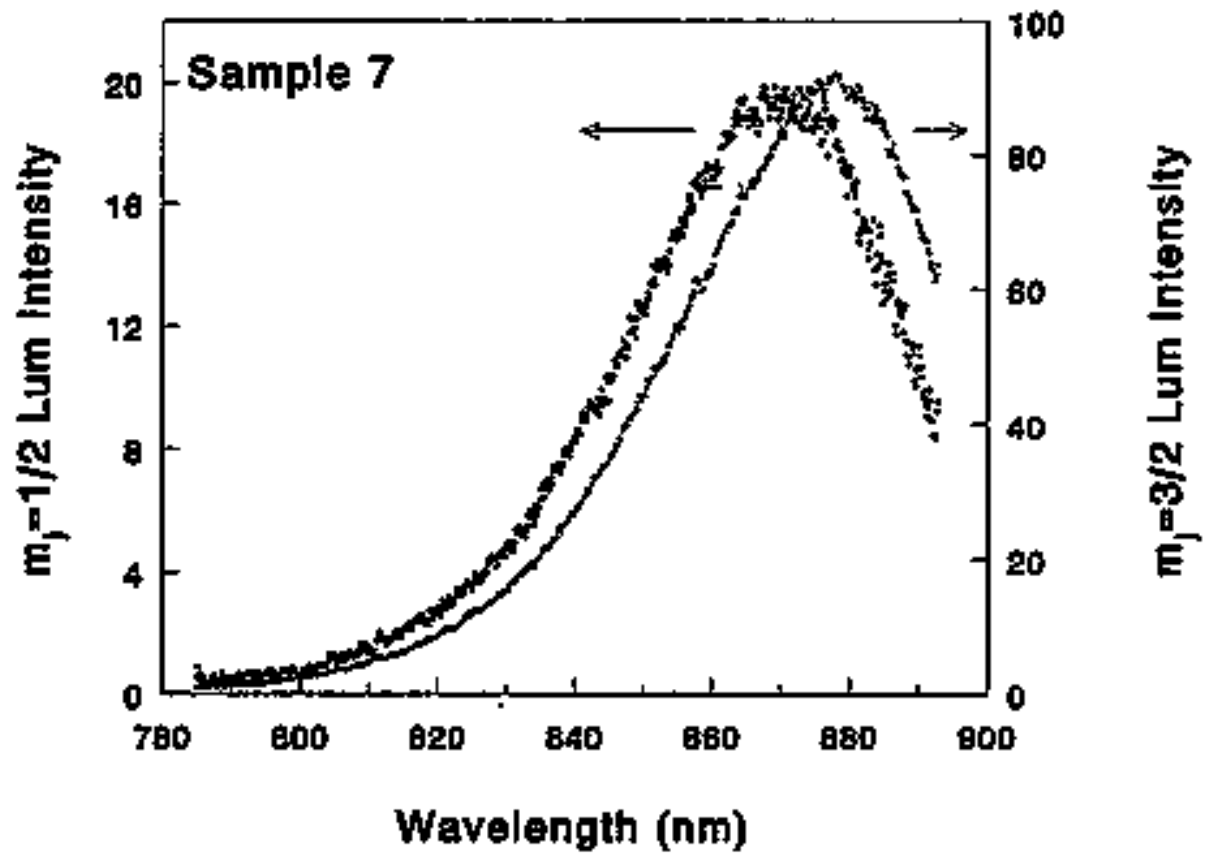

Figure 6.2: Deconvolved luminescence spectra from the two $J=3 / 2$ valence.bands for sample \#7 at room temperature. 
ing circular polarization curves used were those shown in Figures 5.9 through 5.17 (excluding those for sample \#8). Table 6.1 lists the values for $S$ used in the calculations and comments on the method of choice for $S$. Figures 6.1 and 6.2 show deconvolved spectra for the most highly strained sample \#1 and the least strained sample \#7, respectively. It is clear that for each of these extremes the deconvolution provides line-shapes for the indjvidual spectra which are similar to each other and to the general line-shape of the non-strained GaAs sample (\#8) shown in Figure 5.16. The deconvolved luminescence spectra for each sample are fit with Log-Normal curves in order to determine the peak positions. However, no attempt was made to assign peak positions to the deconvolved spectra for sample \#2 because of the interference pattern present in the luminescence spectrum.

\subsection{Calculation of Deformation Potentials for GaAs}

\subsubsection{Deformation Potential b}

The peak positions of the deconvolved luminescence spectsa from the previous section may be used to determine the deformation potential related to the valeace band splitting under a biaxial compressive strain. Using the Gamma point energies of the strain split $J=3 / 2$ valence bands given in Chapter 2, the energy splitting of the bands is

$$
E_{3 / 2,3 / 2}-E_{3 / 2,1 / 2}=-\delta_{s}\left(1+\frac{\delta_{s}}{2 \Delta}\right),
$$

where $\delta_{t}$ was given in eq. $(2.28)$ as

$$
\delta_{s}=-2 b \frac{c_{11}+c_{12}}{c_{11}} \epsilon_{12 x} .
$$




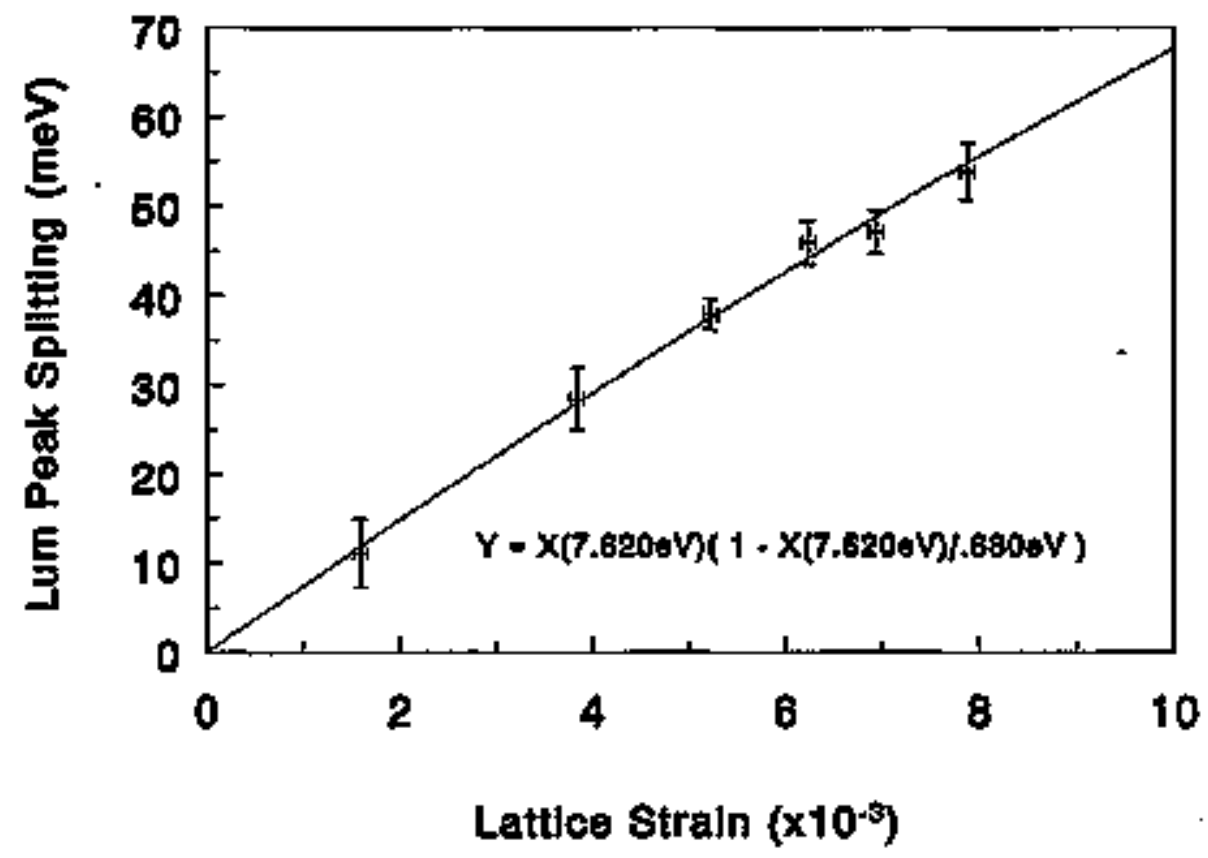

Figure 6.3: Measured $\left.\} \frac{3}{2}, \frac{1}{2}\right\rangle-\left\{\frac{3}{2}, \frac{3}{2}\right\rangle$ luminescence peak splitting plotted against biaxial compressive strain for six of the $\mathrm{GaAs} / \mathrm{GaAs}_{(1-x)} \mathrm{P}_{x}$ samples. The solid line is a weighted least squares fit of eg. (6.6) to the data.

Figure 6.3 shows a plot of the measured splitting of the deconvolved luminescence spectra v8. the in-plane lattice strain as measured by X-ray diffraction. There are data points for jix of the seven GaAs/GaAs(1-x) $\mathrm{P}_{x}$ photocathode samples. Sample \#2 was not used because of the interference pattern superimposed on the luminescence spectrum. The data is plotted with an uncertainty in lattice strain of $\pm 7 \times 10^{-5}$ which is due to an estimated uncertainty in (004) X-ray diffraction curve splitting of $\pm .005^{\circ}$ in $2 \theta$. The uncertajnty in luminescence peak splitting is calculated as the sum of the estimated $\pm 1.4 \mathrm{meV}$ uncertainty (random) in luminescence peak energy difference and the observed sensitivity in splitting energy (systematic) to choice of $S$. The solid line (and equation) shown in Figure 6.3 is a least squares 
fit of a curve with the form of eq. (6.6). The value of $\Delta$ was taken as $340 \mathrm{meV}[59]$ for the purpose of determining the fit. Keeping in mind that the strains plotted in Figure 6.3 are actually segative, we find a value of $(-7.620 \mathrm{eV}) \epsilon_{x x}$ for $\delta_{s}$. Using values of $c_{11}=11.88 \times 10^{11} \mathrm{dyn} / \mathrm{cm}^{2}$, and $c_{12}=5.38 \times 10^{11} \mathrm{dyn} / \mathrm{cm}^{2}[59]$ and solving eq. (6.7), we find a value for the GaAs deformation potential of $b=-2.00 \pm .05$ $\mathrm{eV}$. This is in excellent agreement with pablished values for $b$ obtained from room temperature measurements.[72] Table 6.3 may be referred to for a summary of several published values.

\subsubsection{Deformation Potential a}

The hydrostatic deformation potential for GaAs may also be determined frem the deconvolved luminescence spectra. The Gamma point band gap energies associated with the two $J=3 / 2$ valence bands are averaged as

$$
E_{c}-\left(\frac{E_{3 / 2,3 / 2}+E_{3 / 2,1 / 2}}{2}\right)=E_{g}(\epsilon=0)+\delta_{h}-\frac{\delta_{s}^{2}}{4 \Delta},
$$

where $E_{y}(c=0)$ is the zero-strain band gap energy. The hydrostatic term was given in eq. (2.27) as

$$
\delta_{k}=2 a \frac{c_{11}-c_{12}}{c_{11}} \epsilon_{s x}
$$

The strain dependence of the averaged $J=3 / 2$ luminescence peak energy is plotted in Figure 6.4. The same six strained GaAs samples used in Figure 6.3 are used here. The peak position for the non-strained sample \#8 is also used in this plot as the zero-strain data point. Also shown in Figure 6.4, is a least squares fit to the data which uses an equation of the form of eq. (6.8) and incorporates the 


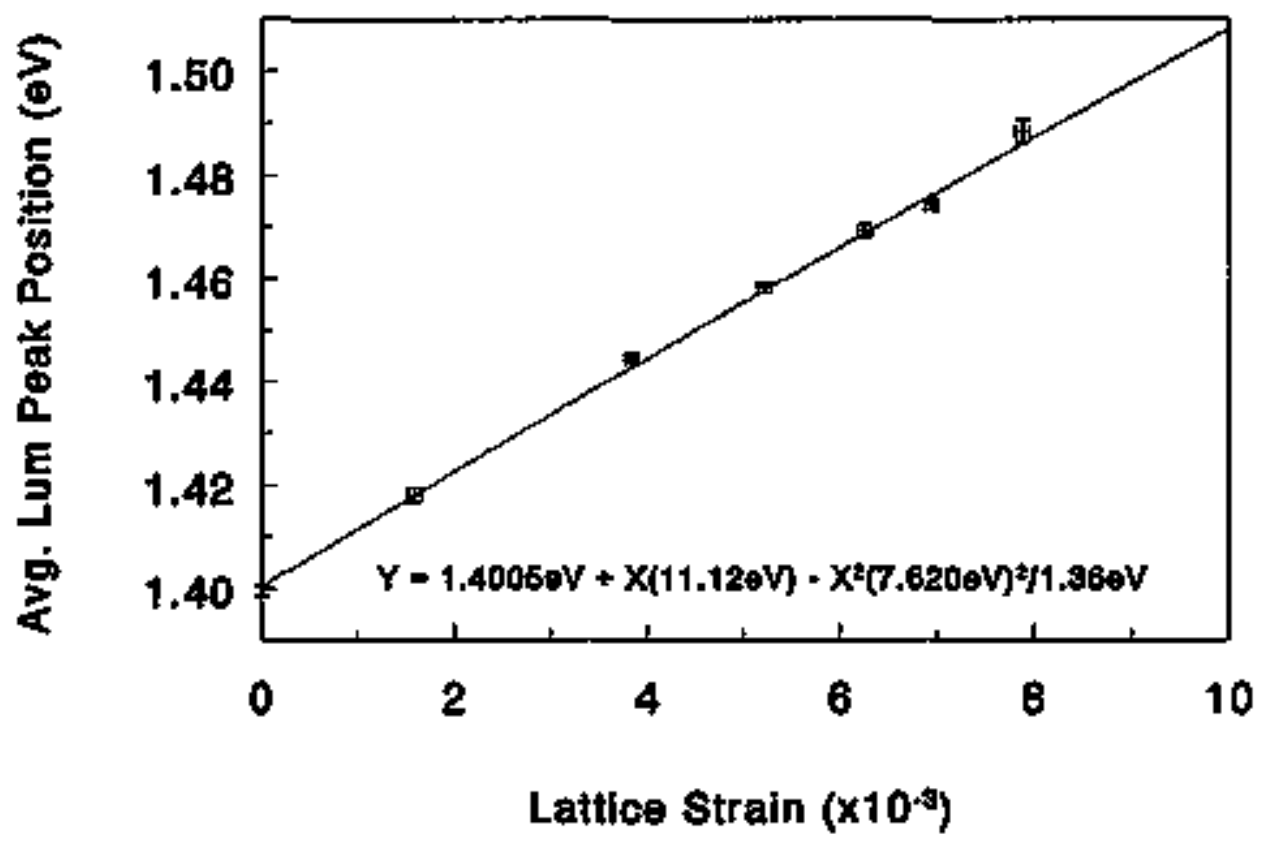

Figure 6.4: Measured $\left|\frac{3}{2}, \frac{3}{3}\right\rangle-\left|\frac{3}{2}, \frac{1}{2}\right\rangle$ average luminescence peak energy plotted against biaxial compressive strain for six of the $\mathrm{GaAs} / \mathrm{GaAs} \mathrm{s}_{(1-x)} \mathrm{P}_{x}$ samples and the non-strajned GaAs sample. The solid line is a weighted least squares fit of eq. (6.8) to the data. 
value of $\delta_{\varepsilon}$ obtained from the plot in Figure 6.3. Using the value for $\delta_{A}$ from the fit (remembering once again that the plotted lattice strains are actnally negative) and solving equation (6.9), we find a value for the hydrostatic deformation potential of $a=-10.16 \pm .2 \mathrm{l} \mathrm{eV}$. Here, the uncertainty is determined by the least squares fit. This is in good agreenent with the published value of $a=-9.77 \pm .08 \mathrm{eV}$.[25]

\subsubsection{Deformation Potential $d$}

Appendix $\mathrm{C}$ discusses the presence of in-plane shear strains in the strained layer photocathodes and the linearly polarized luminescence asymmetry which results from such a shear. Measurements of the in-plaze shear and linearly polarized luminescence asymmetry are also provided. These measurements may be used in eq. (C.6) to calculate a value for the shear deformation potential $d$. The maximum asymmetry for a sample is taken as the magnitude of the $\sin 2 \phi$ fit to the linearly polarized luminescence asymmetry data. The uncertainty in this value is estimated to be $\pm 5 \times 10^{-3}$ (absolute) based upon measurements from all eight etched samples. The valence band energy splittings needed for $\delta_{1}$ and $\delta_{2}$ in eqs. (C.7) and (C.8) are $E_{3 / 2,3 / 2}-E_{3 / 2,1 / 2}=-\delta_{s}-\delta_{s}^{2} / 2 \Delta$ and $E_{3 / 2,3 / 2}-E_{1 / 2,1 / 2}=\Delta-\delta_{s} / 2+\delta_{4}^{2} / 2 \Delta$, where $\delta$, was defined in eq. (2.28). When calculating $d$, the value of the deformation potential $b$ found in Section 6.2.I is used.

The measurements of linear polarization asymmetry and in-plane shear for sample \#4 yield a value of $d=-5.04 \pm .92 \mathrm{eV}$, while those for sample \#5 yjeld a value of $d=-4.85 \pm .31 \mathrm{eV}$. The uncertainty associated with each value is determined by the uncertainties in $b, \epsilon_{x y}$ and $A^{\text {max }}$. The combined value for the 
deformation potential $d$ of GaAs is $d=-4.87 \pm .29 \mathrm{eV}$, which is in reasonable agreement with other published values from room temperature measurements.[72] Table 6.3 may be referred to for a summary of several published values.

\subsection{Characteristics of the Spin Relaxation Factor $R$}

Comparison of room temperature Juminescence circular polarization data with 78 $\mathrm{K}$ and $12 \mathrm{~K}$ data in Chapter 5 implied that the spin relaxation factor $R$ is temperature independent for the GaAs strained layer samples. In order to directly compare values for different samples, the room temperature spin relaxation factor is calculated for each of the samples studied. The values for $R$ are estimated from the room temperature luminescence circular polarization data shown in Figures 5.19 through 5.27. Also calculated for each sample is the normalized luminescence intensity. This quantity is taken as the observed luminescence peak maximum corrected for total run time, pump power and approximate pump beam absorption. As defined, it is proportional to luminescence efficiency. For the absorption correction, the nominal active layer thicknesses are used and the absorption coefficient is taken as $1.5 / \mu \mathrm{m}[73]$ to represent pump wavelengths in the vicinity of 750 nm to $780 \mathrm{~nm}$. Table 6.2 lists the values of the quantities $R$, normalized intensity and lattice strain $\left(\epsilon_{x s}\right)$ for the nine samples studied. The measured spin relaxation factors for the various samples are believed to be independent of the sample preparation technique of substrate etching and application of DAG because the non-thinned sample (\#9) exhibits a relaxation factor which falls in the range of those given for etched samples of similar strain and luminescence efficiency. 
Normalized Lattice Strain

\begin{tabular}{cccc} 
Sample \# & $R$ & Lum. Intensity & $\left(\times 10^{-3}\right)$ \\
\hline 1 & .636 & 394 & -7.88 \\
2 & .615 & 189 & -6.99 \\
3 & .750 & 903 & -6.94 \\
4 & .660 & 304 & -5.22 \\
5 & .680 & 381 & -6.24 \\
6 & .735 & 713 & -3.84 \\
7 & .815 & 7260 & -1.59 \\
8 & .832 & 2952 & 0.0 \\
9 & .690 & 468 & -4.2 \\
\hline
\end{tabular}

Table 6.2: Estimated values of the spin relaxation factor $R$, the normalized luminescence peak intensity and the lattice strain $\left(\epsilon_{x x}\right)$ for the photocathode samples. The $\left(^{*}\right)$ indicates the value of lattice strain for sample \#9 is that estimated in Appendix B. 
One may make a rough estimate of the expected effect of known spin relaxation rates for 'bulk' p-type GaAs on the thin GaAs layers. Values of spin relaxation time and electron lifetime have been experimentaliy determined for GaAs doped

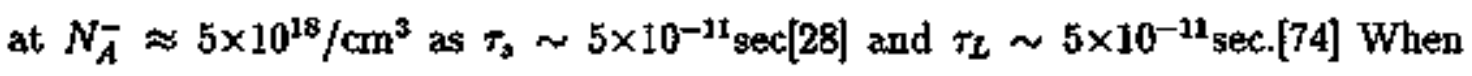
one considers the order of magnitude estimate of electron lifetime in an epitaxial GaAs sample of $100 \mathrm{~nm}$ thickness to be approximately $\tau_{Z}\left(\right.$ epi) $/ \tau_{Z}$ (bulk) $\sim 0.01$ as evidenced in relative luminescence efficiencies, the spin relaxation factor expected from eq. (3.28) is $R \sim .99$. Therefore, the degree of spin relaxation observed for the samples in this study cannot be explained by the spin relaxation rates given for 'bulk' GaAs.

Figure 6.5 shows the relaxation factor plotted against normalized luminescence intensity. It is very interesting to note that $R$ actually appears to increase with increasing normalized luminescence intensity. This is counter-intuitive to the classic relaxation mechanisms which are characterized by a spin decay time $\tau_{s}$. This can be seen by considering the expressions for $R$ and luminescence efficiency ( $\epsilon_{i t m}$ ). First, the relaxation factor for a classic 'bulk' process is expressible as

$$
R=\frac{\tau_{s}}{\tau_{s}+\tau_{L}},
$$

and the luminescence efficiency as

$$
\epsilon_{\mathrm{lum}}=\frac{\tau_{L}}{\tau_{R}}
$$

Here, $\tau_{L}$ is the minority electron lifetime and $\tau_{R}$ is the radiative recombination lifetime. The expression for $R$ may be rewritten as

$$
R=\frac{\tau_{s}}{\tau_{s}+\tau_{R^{\prime}} \epsilon_{\text {lum }}}
$$


where $\tau_{R}$ may be considered a constant for fixed doping level. Equation (6.12) shows that, for a given relaxation mechanism (and $\tau_{s}$ ), one expects greater relaxation (smaller $R$ ) with greater luminescence efficiency. Because Figure 6.5 shows an opposite trend, it does not support the notion of a 'bulk' spin relaxation process characterized by a spin decay time. Rather, it supports the idea of a spin relaxation process associated with the surface or the surface depletion region. This is because the samples with lower luminesceace efficiency presumably have a larger fraction of radiatively recombining electrons which have spent time near the surface of the sample. Here, the relative luminescence efficiency is dependent upon a combination of active layer thickness and surface recombination velocities at both the surface and the $\mathrm{GaAs}_{\mathrm{S}} / \mathrm{GaAs}_{(1-x)} \mathrm{P}_{x}$ interface as was discussed in Section 4.3.2. The surface depletion region (band bending region) for a NEA activated photocathode is seen in Figure 1.1 presented in Chapter 1. Similar band bending occurs at the surface of p-type GaAs without NEA activation as a result of a high density of surface states near mid-gap related to adsorbed oxygen.[75]

Figure 6.6 shows the relaxation factor plotted against lattice strain. This plat appears to show a trend of increasing spin relaxation (smaller $R$ ) with increasing strain. However, deviations from the trend are numerous in this plot. One might attempt to attribute the scatter in the plot to variation of $\tau_{L}$. For example, given two samples of similar strain, a difference in $R$ might be due to a difference in $\tau_{L}$ rather than the (presumably, here) strain dependent $\tau_{s}$. According to eq. (6.12), the sample exhibiting less spin relaxation (larger $R$ ) would need to exhibit a shorter electron lifetime, observable as a smaller luminescence efficiency, for this argument 


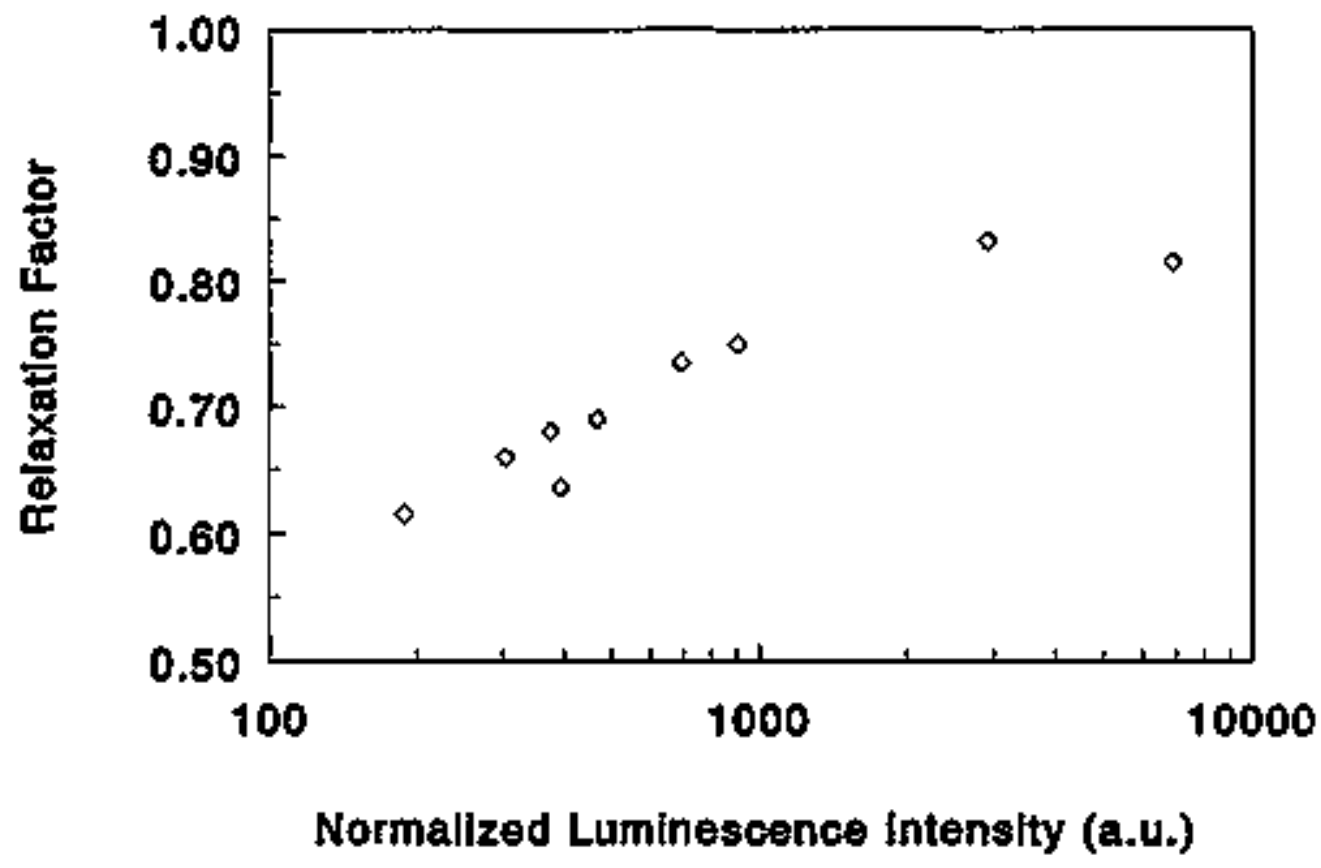

Figure 6.5: Spin relaxation factor $R$ plotted against the normalized luminescence intensity (proportional to luminescence efficiency) for the photocathode samples. 


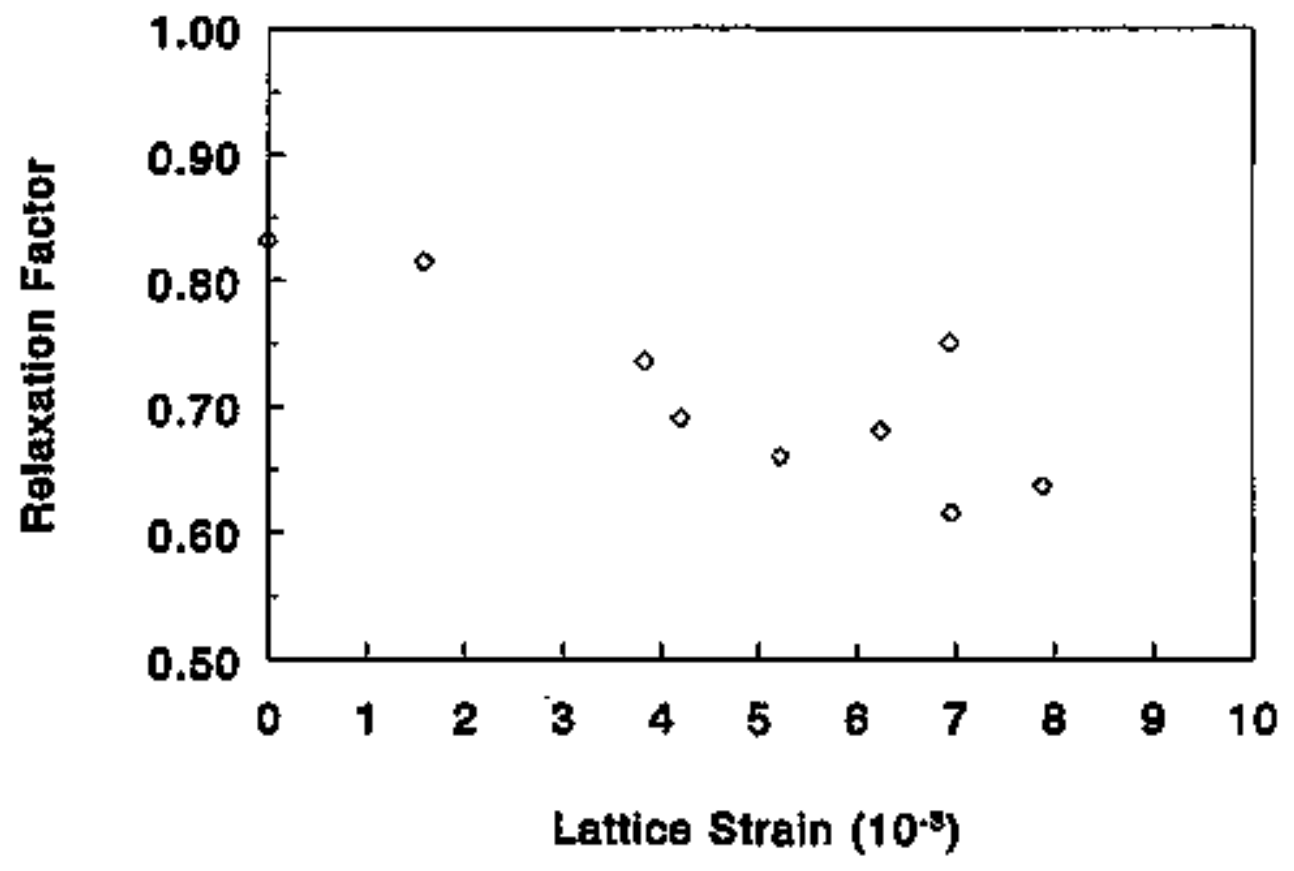

Figure 6.6: Spin relaxation factor $R$ plotted against lattice strain $\epsilon_{x x}$ for the photocathode samples. 
to be valid. However, upon inspection of the data given in Table 6.2, this is not the case. In fact, compensation for variation in $\tau_{L}$ acts to increase the scatter observed in Figure 6.6. Therefore, the dat do not support a strain dependent spin relaxation factor.

\subsection{Conclusions}

The luminescence data presented within this work support a model for strainenhanced electron spin polarization in which the strain split valence bands exhibit polarizations of $\pm 100 \%$ under the condition of optical pumping with circularly polarized light in the vicinity of band edge. This appears valid for photocathode samples doped at the level of $N_{\mathcal{A}}^{-}=5 \times 10^{18} / \mathrm{cm}^{3}$ with lattice strains as small as $\epsilon_{x x}=-1.59 \times 10^{-3}$. Clearly, the near- $\Gamma$ valence band wave functions and associated electron spin polarization values must approach those for the non-strained case as the lattice strain goes to zero. The way in which this transition occurs, however, is likely dependent upon doping level and the associated band tailing.

The presence of band tails destroys the parabolicity of the bands and affects the wave functions near $\vec{k}=0$ with electron localization effects.[62] This makes any calculation of the expected electron spin polarization extremely difficult in the limit where strain dependent and $\vec{k}$ dependent terms in the $k \cdot p$ matrix are comparable. An estimate of the $\vec{k}$ dependent effects in the absence of band tailing has been made and it was determined that the electron polarization for transitions from the $m_{j}=3 / 2$ valence band was not strongly affected $\left(P_{\mathrm{t}}-\geq 95 \%\right)$ for photon energies less than the $m_{j}=1 / 2$ basd gap. One could argue that the presence of band 
tails actually reduces the effects of $\vec{k}$ dependent terms competing agajnst straindependent terms because the potential fuctuations which make up the tails destroy the free electron wave nature of low kinetic energy valence electrons. Luminescence experiments performed with strained layer photocathode sets of varied doping level might prove useful in better understanding the role of band tailing in strained layer photocathodes.

With the assumption of $\pm 100 \%$ polarizations from the two uppermost valeace bands, measurements of the deformation potentials $a, b$ and $d$ for GaAs were made. The measured values are shown in Table 6.3 compared with several published values. The published values provided were stmmarized in [76] with the exception of the value for a marked by (*), which was found in [25]. The values determined in this study are in good agreement with those previously published. We believe the method of these measurements to be unique. Provided the methods used to extract the values are valid, the measurement of $b$ is more precise than many previous measurements.

Spin relaxation factors for the photocathode samples were estimated from the luminescence data. Study of the spin relaxation factor is consistent with the hypothesis that the degree of spin relaxation in the samples is temperature insensitive and thus not due to one of the classic relaxation mechanisms associated with the 'bulk'. Sample to sample comparison of the spin relaxation factor indicates that it may be related to relaxation within the depletion region or at the surface. Such surface related spin relaxation has previously been reported.[77] Spin redaxation near the sample surface may also explain observations of a quatum efficiency 


\begin{tabular}{cccc}
$a(\mathrm{eV})$ & $b(\mathrm{eV})$ & $d(\mathrm{eV})$ & \\
\hline$-10.16 \pm .21$ & $-2.00 \pm .05$ & $-4.87 \pm .29$ & this work \\
\hline$-9.77 \pm .08^{*}$ & & & previously published \\
$-8.7 \pm .4$ & $-2.1 \pm .1$ & $-6.5 \pm .3$ & $\cdot$ \\
& $-1.7 \pm .2$ & $-4.4 \pm .5$ &. \\
& $-2.0 \pm .1$ & $-5.4 \pm .3$ &. \\
& $-2.0 \pm .2$ & $-6.0 \pm .4$ &. \\
& $-2.0 \pm .2$ & $-5.3 \pm .4$ &. \\
& $-1.75 \pm .3$ & $-5.55 \pm 1.1$ &. \\
$-8.93 \pm .9$ & $-1.76 \pm .1$ & $-4.59 \pm .25$ &. \\
$-8.14 \pm .8$ & $-1.66 \pm .1$ & $-4.52 \pm .25$ &. \\
\hline
\end{tabular}

Table 6.3: Comparison of measured deformation potentials with previously published values for GaAs. 
(QE) dependence on photo-emitted electron spin polarization.[67][78] In these experiments, the photo-enitted electron spin polarization from GaAs photocathode structures is seen to increase as the QE decreases. Lower $\mathrm{QE}$ may be the result of a higher vacuum level (relative to the Fermi level) which prevents the lower energy electrons that have thernalized (and experienced spin relaxation) in the band bending region from escaping.

The idea that spin relaxation occurs in the surface band bending region is further supported by luminescence circular polarization measurements which have been performed on $100 \mathrm{~nm}$ thick layers of p-type $\left(N_{A}=5 \times 10^{18} / \mathrm{cm}^{3}\right) \mathrm{Al}{ }_{10} \mathrm{Ga} .90 \mathrm{As}$ clad on both sides with $\mathrm{Al}_{.15} \mathrm{Ga}_{\text {.65 }} \mathrm{As}$.[79] These structures were grown by MBE for the purpose of a separate luminescence study. The layers do not have a free surface and thus no band bending region. Room temperature circular polarization measurements performed on these samples, with the same apparatus used for the current study, yjelded values of $P_{\gamma} \geq 23.5 \%$. This corresponds to a spin relaxation factor of $R \approx .94$, which indicates much less spin relaxation than observed for all of the samples of the current study.

A possible explanation for surface related depolatization may be an increase of the efficjency of the 'classic' relaxation mechanisms within the band bending region. For example, the Elliott-Yafet process depends upon the $\vec{k}$ dependent mixing of conduction and valence $m_{j}=1 / 2$ states. In the band bending region, electrons may be accelerated towards the surface and the larger valtes of $\vec{k}$ will cause greater mixing of the conduction and valence states. The strength of this mechanism has a quadratic dependence on kinetic energy, [29] thus increased kinetic 
energy in the band bending region can have a strong effect on the spin relaxation rate. The band bending region may also contribute new relaxation terms not present in the 'bulk'. For example, the electric field $(E)$ within the band bending region should cause Stark-like mixing of the conduction band wave functions with $m_{j}=1 / 2$ valence band wave functions. This mixing is expected to be of the form

$$
\langle i S \dagger(\downarrow)|E z| Z \uparrow(\downarrow)\rangle
$$

and it may result in spin relaxation in the same way the $\tilde{k}$ depentent mixing does within the E-Y mechanism.

\subsubsection{Possible Improvements to Photocathode Structure}

Considering the observations made herein, suggestions may be made with regard to future photocathode design. First, the model used to describe the data accounts for less than $100 \%$ electron polarization from a strained layer photocathode by assuming that the condition of purely selective pumping from the uppermost valence band is not met. This is assumed to be a consequence of the presence of band tails. In the interest of greater obtainable electron spis polarization, the degree of band tailing within a photocathode may be reduced with a reduction in doping level. High p-type doping is necessary, however, for activation to NEA. These competing needs may both be met by designing a photocathode with a reduced doping level (i.e. $5-10 \times 10^{17} / \mathrm{cm}^{3}$ ) in the majority of the active layer and a high doping level $\left(5-10 \times 10^{18} / \mathrm{cm}^{3}\right)$ in approximately the last $100 \AA$. In this way, the majority of the actjve layer contributing to optical absorption has a reduced level of band tailing while the condition of NEA is maintained. Structures with such modulated 
doping profiles bave previously been proposed. In fact, spin polarization measurements from a set of GaAs-AlGaAs (superlattice) photocathode structures of similar design parameters but varied doping level have been made, $[80]$ indicating that the polarization does improve with decreased doping level in the 'bulk' of the photocathode.

A second possible improvement in photocathode design may be to devise a method of reducing or eliminating the conduction band bending region while maintaining a NEA surface. One way to reduce the width of the band bending region is to increase the doping level near the surface with a modulated doping scheme similar to that already described. Another, more exotic, possibility may be to compensate the downward bending of the conduction band by incorporating, at the surface, a thin layer of an increasing band gap alloy such as $\mathrm{Al}_{x} \mathrm{Ga}_{(1-x)} \mathrm{As}$. For example, the Al content can be taken from 0 to $\sim 0.25$ in the last 50-100 Aof the photocathode active layer in order to provide a band gap which increases by $\sim 0.4 \mathrm{eV}$ near the surface. $\mathrm{Al}_{x} \mathrm{Ga}_{(1-x)} \mathrm{As}$ is nearly lattice matched to GaAs, so concerns of strain or strain relaxation induced effects are avoided. Figure 6.7 shows schematically such a structure. The effect of the increasing band gap is to provide a reduced level of conduction band bending while still providing the charge transfer at the surface which is necessary for NEA. The near-surface conduction band edge is shown with a non-srnooth curvature in Figure 6.7 to illestrate imperfect compensation of the band bending by the graded $\mathrm{Al}_{x} \mathrm{Ga}_{(1-x)}$ As layer. This proposed structure is expected to reduce both the width and magnitude of the conduction band bending region. Therefore, if the spin depolarization rate within the band 


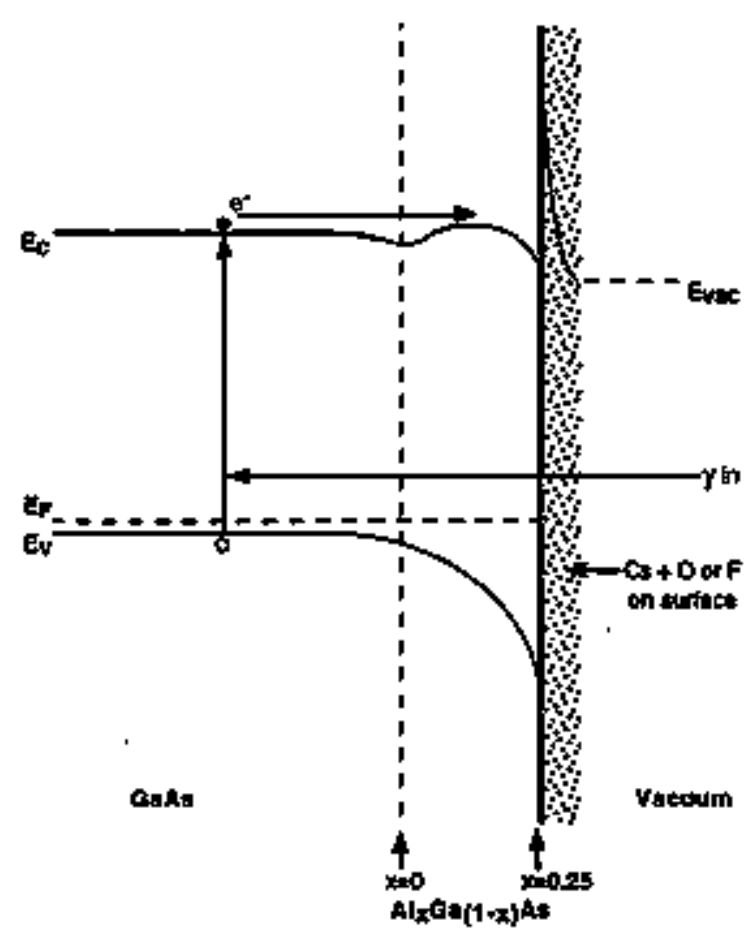

Figure 6.7: Diagram of an NEA surface for a photocathode with a graded $\mathrm{Al}_{x} \mathrm{Ga}_{(1-x)} \mathrm{As}(x=0$ to, 25 ) layer at surface to compensate for downward bending of the conduction band.

bending region depends on the electron kinetic energy, this structure should reduce the level of electron spin depolarization. 


\section{Appendix A}

\section{Calculation of Electron Spin Polarization Ùsing}

\section{Cubic Symmetry}

The $50 \%$ polarization result obtained for non-strajned GaAs in Chapter 3 used an assumption of spherically symmetric constant energy surfaces for the conduction and valence bands in order to facilitate summation over all $\vec{k}$ directions. This assumption is not necessary, though. The cubic symmetry of GaAs is suffeient to insure the $50 \%$ result after summation. This can be shown with a fairly simple argument. The goal is to sum the spin density matrix elements over all allowed transitions for a given photon energy. If one considers an allowed wave vector $\vec{k}=k_{i} \hat{x}+k_{j} \hat{y}+k_{k} \hat{z}$, then cubic symmetzy insures degeneracy with all wave vectors of the form

$$
\begin{aligned}
& \pm k_{i} \hat{x} \pm k_{j} \hat{y} \pm k_{k} \hat{z}, \pm k_{j} \hat{x} \pm k_{k} \hat{y} \pm k_{j} \hat{z}, \pm k_{j} \hat{x} \pm k_{k} \hat{y} \pm k_{i} \hat{z}, \\
& \pm k_{j} \hat{x} \pm k_{i} \hat{y} \pm k_{k} \hat{z}, \pm k_{k} \hat{x} \pm k_{i} \hat{y} \pm k_{j} \hat{z}, \pm k_{k} \hat{x} \pm k_{j} \hat{y} \pm k_{i} \hat{z} .
\end{aligned}
$$

The spin density matrix elements may be summed over these degenerate wave vectors with the knowledge that they each have the same population density. The equation for spin polarjzation may be expressed in a more convenient form for the 
summations that will be made.

$$
\vec{P}_{c^{-}}^{(n)}=\frac{\operatorname{Tr}\left(F^{(n)} \tilde{\sigma}\right)}{\operatorname{Tr}\left(F^{(n)}\right)}=\frac{\sum \operatorname{Tr}\left(f^{(n)} \ddot{\sigma}\right)}{\sum \operatorname{Tr}\left(f^{(n)}\right)}=\frac{\sum_{j}^{\text {sector }} \sum_{\text {degen. }} \operatorname{Tr}\left(f^{(n)} \vec{\sigma}\right)}{\sum_{i}^{\text {sector }} \sum_{\text {degen. }} \operatorname{Tr}\left(f^{(n)}\right)}
$$

The summation $\sum_{\text {degen. }}$ is over all degenerate states from eq. (A.1) for an allowed wave vector of a specific $k_{i}, k_{j}, k_{k}$ and the summation $\sum_{i}^{\text {sector }}$ is over all allowed wave vectors within a full sector of non-degenerate (by symmetry) wave vectors. Therefore, the total summation is over all allowed transitions.

The absorbed photon may be defined along a direction $\hat{z}^{\prime}$, and the spin polarization along this direction will be calculated for each valence band. Combining eqd. (3.9) and (3.10) with eq. (A.2), the spin polarization for transitions from each valence band may be written as

$$
\left(P_{e^{-}}^{(1)}\right)_{\bar{z}^{\prime}}=\frac{\sum_{i}^{\text {sector }} N_{i} \sum_{\text {degan. }}\left(-2 \cos ^{2} \theta\right)}{\sum_{i}^{\text {stetor }} N_{i} \sum_{\text {degen }}\left(1+\cos ^{2} \theta\right)}
$$

and

$$
\left(P_{\mathrm{e}^{-}}^{(2)}\right)_{\hat{i}^{\prime}}=\frac{\sum_{i}^{\text {gector }} N_{t} \sum_{\text {degen. }}\left(2-6 \sin ^{2} \theta\right)}{\sum_{i}^{\text {sector }} N_{i} \sum_{\text {degen. }}\left(2+3 \sin ^{2} \theta\right)}
$$

Here, $\theta$ is the angle between $\hat{z}^{\prime}$ and the wave vector $\vec{k}$. The angular dependesce in eqs. (A.3) and (A.4) may be expressed purely in terms of $\cos ^{2} \theta$ which, in turn, may be expressed as the square of the projection of $\vec{k}$ onto $\hat{z}^{\prime}$. In this manner, the polarizations given in eqs. (A.3) and (A.4) are rewritten as

$$
\left(P_{e^{-}}^{(1)}\right)_{s^{\prime}}=\frac{\sum_{j}^{\text {sector }} N_{i} \sum_{\text {degta. }}\left(-2 k_{z^{\prime}}^{2} / k^{2}\right)}{\sum_{i}^{\text {sector }} N_{i} \sum_{\text {depen. }}\left(1+k_{z^{\prime}}^{2} / k^{2}\right)}
$$

and

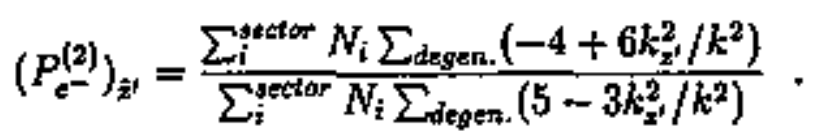


The term $k_{x^{\prime}}$ in eqs. (A.5) and (A.6) is related to the wave vectos in the $\hat{x}, \hat{y}, \hat{z}$ coordinate system by the rotation matrix given in eq. (2.16) and $k_{z^{\prime}}^{2}$ is expressed in terms of the wave vector components as

$$
k_{z^{\prime}}^{2}=k_{z}^{2} \sin ^{2} \theta \cos ^{2} \phi+k_{y}^{2} \sin ^{2} \theta \sin ^{2} \phi+k_{z}^{2} \cos ^{2} \theta
$$

Of course, the subscripts $x, y, z$ which correspond to the wave vector coordinate system will be subject to all permutations of the wave vector subscripts $i, j, k$ when summation is made over a group of degenerate wave vectors with components $k_{i}, k_{j}, k_{k}$.

When eq. (A.7) is used in eqs. (A.5) and (A.6) and summation over the degenerate wave vectors is performed, the result is

$$
P_{e^{-}}^{(1)}=P_{\epsilon-}^{(2)}=\frac{\sum_{i}^{\text {yector }} N_{i}\left(-c_{i}\right)}{\sum_{i}^{\text {sector }} N_{i}\left(2 c_{j}\right)}=-0.5 \frac{\sum_{i}^{\text {sector }} N_{i} c_{i}}{\sum_{i}^{\text {settor }} N_{i} c_{i}}=-0.5 .
$$

The terms $c_{i}$ represent the results of the summations over groups of allowed degenerate wave vectors and are always simple numeric constants. Equation (A.8) shows that the cubic symmetry of GaAs provides the appropriate degeneracies, as listed in eq. (A.1), to insure the $50 \%$ spin polarization result without need for simplifying assumptions of the nature of the constant energy surfaces. 


\section{Appendix B}

\section{Remarks About Non-Thinned Strained Layer}

Sample \#9

Sample \#9 was included in this study because it was grown on a substrate of $\mathrm{GaP}$ rather than GaAs and luminescence from the active layer could be observed without the need for substrate etching. Most luminescence data from this sample was similar to that found for the other strained layer photocathodes. The magnitude of luminescence polarization observed was consistent with other samples indicating that the necessary process of thinging and subsequent application of DAG did not affect observed polarization levels. This sample also showed the observed 'dip' region in the low temperature pump wavelength dependent polarization curves, indicating that this phenomenon was not caused by sample preparation techniques.

One difference between the data from this sample and that from the thinned samples was the position of the luminescence peak when considering the magnitude of $\mathrm{X}$-ray measured lattice strain. The peak appeared at larger energy (shorter wavelengths) than expected. In order to explain the observed deviation, deconvolution of the luminescence spectra was performed as described in Section 6.1. Then, using the approximate $\left|\frac{3}{2}, \frac{3}{2}\right\rangle-\left|\frac{3}{2}, \frac{1}{2}\right\rangle$ peak splitting and the deformation po- 
tential $b$ determined in Section 6.2 .1 , a necessary lattice strain of $\epsilon_{x z} \approx-4.2 \times 10^{-3}$ was inferred. Next, the average $\left|\frac{3}{2}, \frac{3}{2}\right\rangle-\left|\frac{3}{2}, \frac{1}{2}\right\rangle$ luminescence peak energy was compared to that expected from the inferred lattice strain. The discrepancy could be explained by assuming a luminescence peak shift due to an approximate $2 \%$ phosphorus content in the active layer ( $\mathrm{GaAs} .98 \mathrm{P} .02$ rather than GaAs). Interpretation of the X-ray data ((004) Bragg reflection) is also affected by a small phosphorus content in the active layer because the strain is calculated by assuming a relaxed lattice constant of that for GaAs. It was found that the X-ray data was also consistent with a $2 \%$ phosphorus contamination and a lattice strain of $\epsilon_{x x} \approx-4.2 \times 10^{-3}$. The structure of the sample consists of a GaAs (nominally) active layer grown on a bufter layer of InGaP, so that phosphorus contamination might be possible in the growth transition from buffer to active layer. This possibility was substantiated by the person who grew the sample, and could be explained by the presence of a broken shutter on the gas phase MBE apparatus when the sample was grown.[81] 


\section{Appendix C}

\section{In-Plane Shear Strain and Linearly Polarized}

\section{Luminescence Asymmetry}

The pseudomorphic growth of a thin layer of (001) GaAs on a layer of material of slightly different lattice constant results, ideally, in a pure biaxial compression of the GaAs layer. It has been found, however, that partially relaxed layers of GaAs may exhibit an anisotropic strain in the growth plane due to asymmetric generation of misfit dislocations along the ortbogonal [110] and [110] directions.[82] This in-plane shear strain $\left(\epsilon_{x y}\right)$ causes mixing between the $\Gamma$ point valence band wave functions. Valence states of $m_{j}= \pm 3 / 2 \mathrm{mix}$ with $m_{j}=\mp 1 / 2$ for non-zero $\epsilon_{\text {wh }}$, and this has been shown to result in an anisotropy in the optical pumping rate with linearly polarized light.[83] The anisotropy is carried in the electric dipole matrjx element for valence band to conduction band transitions and is therefore also present in the corresponding radiative recombination transitions. It is observable as a measured linear polarization component in the luminescence spectrum. The linear polarization asymmetry $(A)$ is defined as

$$
A(\phi)=\frac{I(\phi)-I\left(\phi+\frac{\pi}{2}\right)}{I(\phi)+I\left(\phi+\frac{\pi}{2}\right)}
$$


where $I(\phi)$ is the fuminescence intensity measured while analyzing for a linear polarization component in the direction defined by the polar angle $\phi$. The asymmetries related to transitions to each of the $J=3 / 2$ valence bands are given as[83]

$$
A^{(1)}(\phi)=\frac{2\left(\delta_{1}+\delta_{2}\right) \sin 2 \phi}{\left(1+\left(\delta_{1}+\delta_{2}\right)^{3}\right)} \cong 2\left(\delta_{1}+\delta_{2}\right) \sin 2 \phi
$$

and

$$
A^{(2)}(\phi)=\frac{-6 \delta_{1} \sin 2 \phi}{\left(1+\delta_{1}^{2}\right)} \cong-6 \delta_{1} \sin 2 \phi,
$$

where $\delta_{1}=d \cdot \epsilon_{x y} /\left(\sqrt{3}\left(E_{3 / 2,3 / 2}-E_{3 / 2,1 / 2}\right)\right)$ and $\delta_{2}=2 d+\epsilon_{x y} /\left(\sqrt{3}\left(E_{3 / 2,3 / 2}-E_{1 / 2,1 / 2}\right)\right)$. The superscripts (1) and (2) zefer, respectively, to the $\left\langle\frac{3}{2}, \frac{3}{2}\right\rangle$ and $\left|\frac{3}{2}, \frac{1}{2}\right\rangle$ valence bands. The two asymmetry terms are opposite in sign suck that, when both transitions are contributing, the asymmetry magnitude will be smaller than for the case of luminescence exclusively from transitions to the $\left.\mid \frac{3}{2}, \frac{3}{2}\right)$ band. In fact, the luminescence wavelength dependence of the linear polarization asymmetry cas be related to that of the circular polarization. The Iuminescence wavelength dependence of the lineas polarization asymmetry is assumed to be due purely to the wavelength dependence of the relative luminescence contribution from each of the $J=3 / 2$ bands, and it is expressed as

$$
A\left(h \nu_{l \mathrm{um}}, \phi\right)=\frac{A^{(1)}(\phi) I^{(1)}\left(h \nu_{l \mathrm{~km}}\right)-A^{(2)}(\phi) I^{(2)}\left(h \nu_{\text {lum }}\right)}{I^{(1)}\left(h \nu_{l \mathrm{~km}}\right)+I^{(2)}\left(h \nu_{\mathrm{lum}}\right)} .
$$

Combining eqs. (C.2)-(C.4) with eqs. (6.3), (6.4) and (6.5), one obtains

$$
A\left(h \nu_{\text {lum }}, \phi\right)=2 \delta_{1}\left(2 \frac{P_{\gamma}\left(h \nu_{\text {lum }}\right)}{S}-1\right) \sin 2 \phi+\delta_{2}\left(\frac{P_{\gamma}\left(h \nu_{l u m}\right)}{S}+1\right) \sin 2 \phi .
$$

The maximum linear polarization asymmetry will occur for $\sin 2 \phi=1$ at the luminescence photon energies (wavelengths) where the circular polarization is also 
maximum. It is given as

$$
A^{\mathrm{max}}=2\left(\delta_{1}+\delta_{2}\right)
$$

Because $\delta_{1}$ and $\delta_{2}$ depend on the shear strain $\epsilon_{x y}$ and the deformation potential $d$, eq. (C.6) may be used to extract a value for $d$ by relating measurements of $\epsilon_{x y}$ and the linear polarization asymmetry.

The terms $\delta_{1}$ and $\delta_{2}$ given in [83] were calculated without consideration of the mixing of the $m_{j}=1 / 2$ valence state induced by the biaxial strain (see eq. (2.26)). The effect of this mixing has only been considered, thus far in this study, in calculation of the Gamma point energies of the valence bands. Because the expected electron spin polazizations from each $m_{j}=1 / 2$ valence band are equivalent under biaxial compressive strain, recalculation of the $m_{j}=1 / 2$ valence states to include the mixing was unnecessary. However, in the interest of performing an accurate meastrement of the deformation potential $d$, the mixing must be considered. This is because the linearly polarized luminescence asymmetry used to measure $d$ depends upon the magnitude of mixing of $(X \pm i Y) \uparrow(t)$ states with the $(X \mp i Y)+(\uparrow)$ components of the $m_{j}=1 / 2$ states. Recalculation of $\delta_{1}$ and $\delta_{2}$ after first order perturbative correction of the $m_{j}=1 / 2$ valence states yields

$$
\delta_{1} \Rightarrow \delta_{1} \cdot\left(\frac{\left[1-\delta_{s} /\left(\Delta+\delta_{s} / 2\right)\right]^{2}}{1+\delta_{s}^{2} / 2\left(\Delta+\delta_{s} / 2\right)^{2}}\right) \cong \frac{d \cdot \epsilon_{x y}}{\sqrt{3\left(E_{3 / 2,3 / 2}-E_{3 / 2,1 / 2}\right)}}\left(1-2 \delta_{s} / \Delta\right)
$$

and

$$
\delta_{2} \Rightarrow \delta_{2} \cdot\left(\frac{\left[1+\delta_{s} / 2\left(\Delta-\delta_{s} / 2\right)\right]^{2}}{1+\delta_{s}^{2} / 2\left(\Delta+\delta_{s} / 2\right)^{2}}\right) \cong \frac{2 d \cdot \epsilon_{x y}}{\sqrt{3}\left(E_{3 / 2,3 / 2}-E_{1 / 2,1 / 2}\right)}\left(1+\delta_{s} / \Delta\right),
$$

where only first order terms of $\delta, / \Delta$ are kept. Equations (C.6), (C.7) and (C.8) are used in Section 6.2 .3 for calculation of the deformation potential $d$. 


\section{C.1 Measurement of In-Plane Shear}

The in-plane shear strain of a sample can be measured with X-ray diffraction techniques. It is defined as

$$
\epsilon_{x y}=\frac{a_{[110]}-a_{[1 \bar{\lambda} 0]}}{a_{[110]}+a_{[1[0]}},
$$

where $a_{[110]}$ and $a_{[1 \bar{z} 0]}$ are the effective lattice constants in the [110] and [110] directions. The growth plane is (001) here. Epilayer (224) reflections used in conjunction with (004) refections provide a measurement of the in-plane lattice constants just as was done for the $\mathrm{GaAs}_{(1-s)} \mathrm{P}_{x}$ layers in Chapter 5. Reciprocal lattice maps, consisting of a series of $\omega / 2 \theta$ scans acquired for different $\omega$ offsets,[84] are used to accurately locate the GaAs epilayer peak relative to the substrate peak. Four reciprocal lattice maps were acquired for each sample being measured: (224), ( $(\overline{2} \overline{2} 4),(2 \overline{2} 4)$, and ( $\overline{2} 24)$, with each providing a value of $d_{h k l}$ from the Bragg equation. The values of $\frac{1}{d k k !}$ are averaged in two pairs in order to cancel out contributions from possible shear strains $\epsilon_{y x}$ and $\epsilon_{x x}$ in eq. (4.6). In this way, $\frac{1}{2}\left(\frac{1}{d_{224}^{2}}+\frac{1}{d_{224}^{2}}\right)$ yields a value of $\frac{1}{d^{2}}$ for calculation of $a_{[120]}$ while $\frac{1}{2}\left(\frac{1}{d_{234}^{2}}+\frac{1}{d_{224}^{2}}\right)$ yields a value for calculation of $\left.a_{[1} \bar{i} i\right]$.

Measurements of $\epsilon_{x y}$ were made for two of the strained layer samples. inplane shear strains of $(1.50 \pm .15) \times 10^{-4}$ and $(5.04 \pm .15) \times 10^{-4}$ were measured for samples \#4 and \#5 respectively. The quoted uncertainties are due to the estimated uncertainties in the difference between (224)/(224) and $(224) /(224)$ epilayer peak positions. The uncertainty in $a_{\perp}$ from the (004) reflection has negligible effect on $\epsilon_{x y}$ 


\section{C.2 Linearly Polarized Luminescence Asymmetry}

The linearly polarized luminescence asymmetry was measured for samples \#1 through \#8. However, only the data from samples \#4 and \#5 are presented because these samples were analyzed for in-plase sbear. The asymmetry is defined by eq. (C.1), so that for any specific $\phi$ it involves luminescence intensity measurements at $\phi$ and $\left(\phi+\frac{\pi}{2}\right)$. In order to analyze linearly polazized luminescence, a second $\lambda / 4$ retarder was placed in the luminescence optics line immediately 'upstream' from the existing retarder/polarizer pair. The angle $\phi$ of the linear polarization analyzer was then determined by the rotation angle of the 'upstream' retarder, and $\left(\phi+\frac{\pi}{2}\right)$ could be easily reached by subsequent $90^{\circ}$ rotation of the 'downstream' retarder. This arrangement allowed for analysis of linearly polarized luminescence while maintaining a fixed polarization state of the luminescence entering the spectrometers. This was necessary because the efficiencies of the spectrometer gratings and mirrors are polarization dependent.

Two types of asymmetry measurement were performed. First, the asymmetry for fixed pump and Juminescence wavelengths was measured for varied orientation ( $\phi$ ) of the linear polarization analyzer. The polarization state of the pump laser was fixed as $\sigma^{+}$and the recorded luminescence wavelength was that which corresponded to the maximum asymmetry. This type of measurement yielded plots which could be fit with $\sin 2 \phi$ carves. Figure C.1 shows plots of this type for samples \#4 and \#5. The samples were aligned relative to the linear polarization analyzer such that $\phi=45^{\circ} / 135^{\circ}$ cotresponded nominally to linear polarization analysis along the crystalline $[1 \mathrm{I} 0] /[110]$ directions, respectively. 

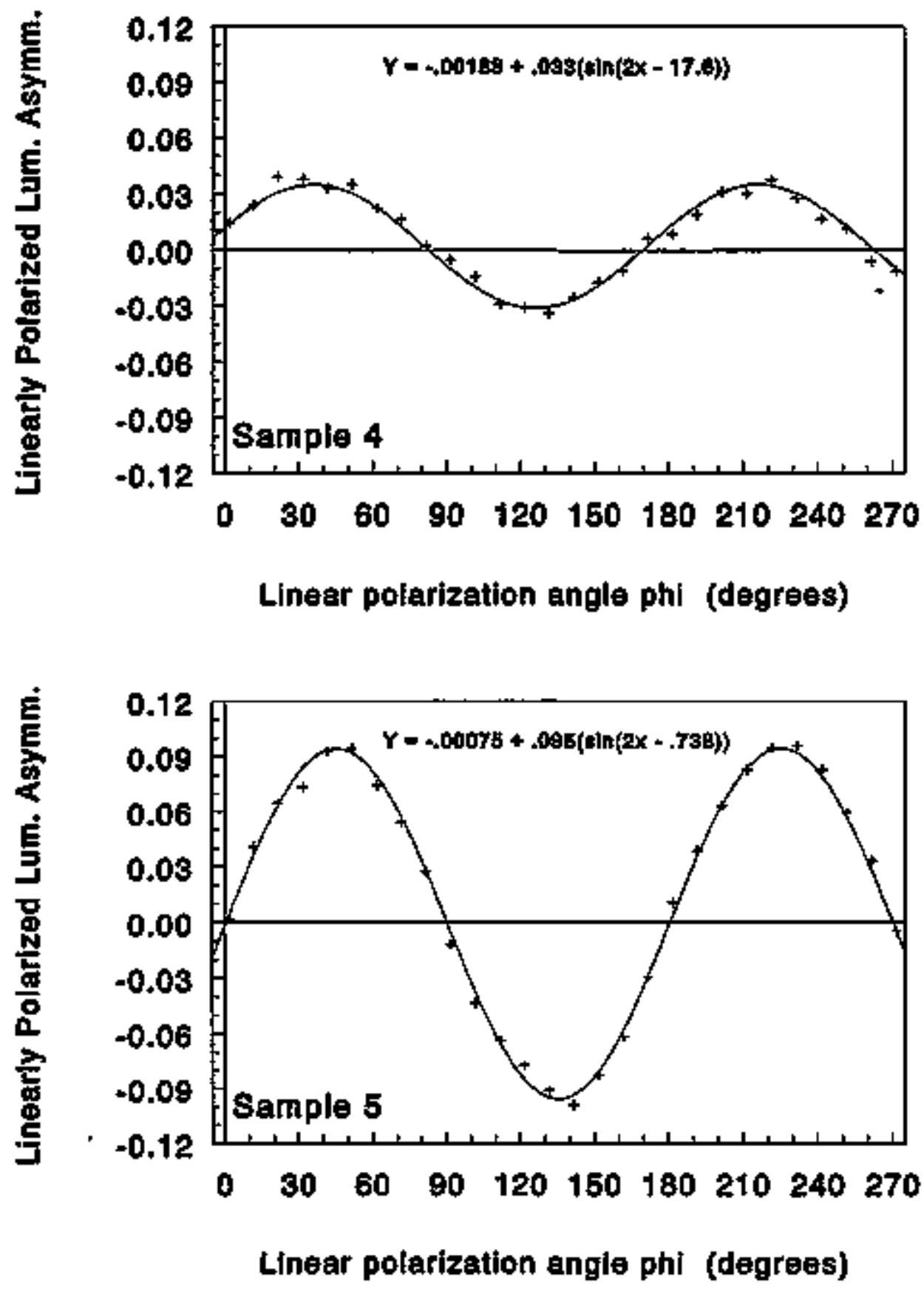

Figure C.1: Linearly polarized luminescence asymmetry for samples \#4 and \#5 plotted against the angle $\phi$ messured relative to the in-plane crystalline axes of the samples. The asyrnmetry is defined by eq. (C.1). Equations and solid lines represent fits to the data. 


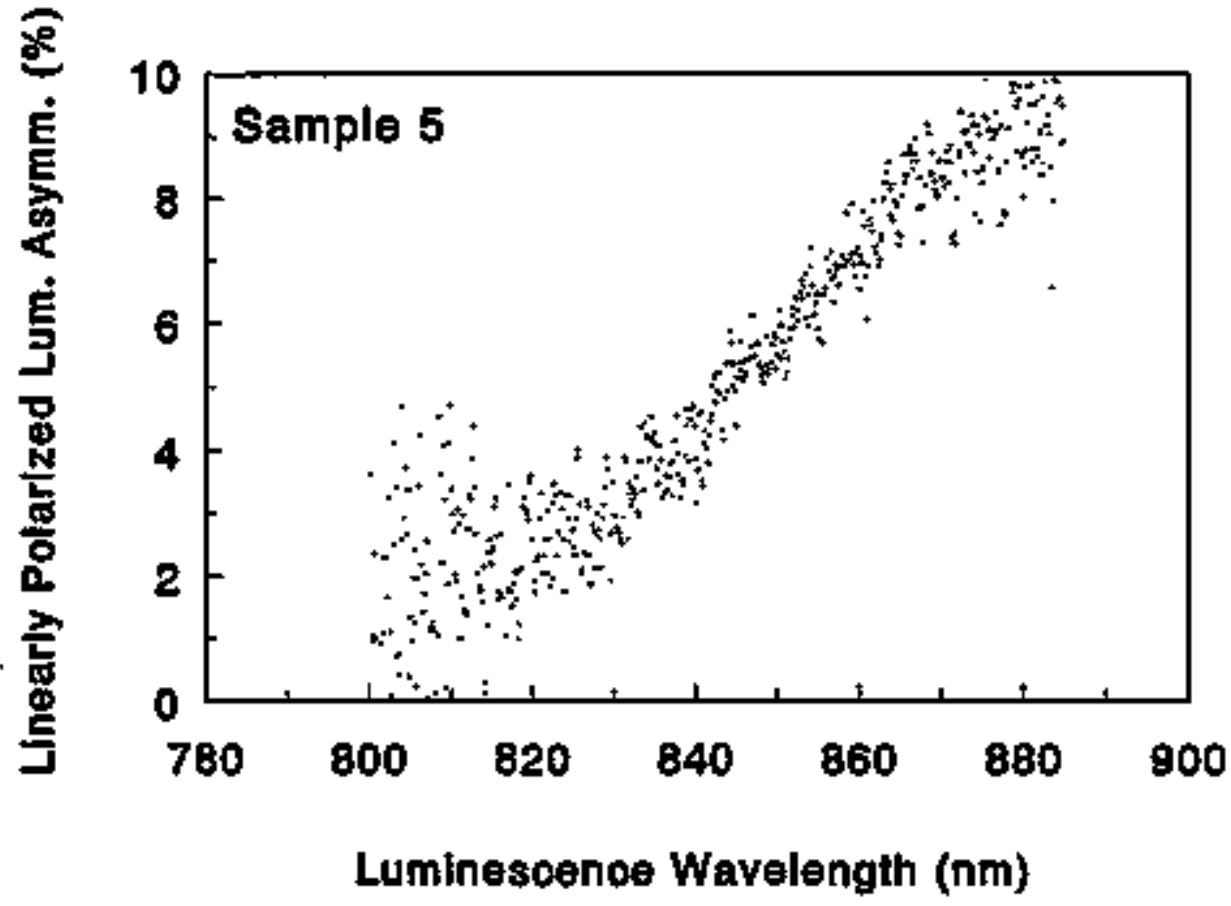

Figure C.2: Luminescence wavelength dependeace of the linearly polarized luminescence asymmetry for sample \#5. 
The second type of measurement consisted of recording the luminescence wavelength dependence of the linearly polarized luminescence asymmetry for the analyzer orientation corresponding to the maximum asymmetry. An example is shown in Figure C.2. Here, the luminescence wavelength depeodent asymmetry for sample \#5 is seen to have a similar structure to the circular polarization curve (Fig. 5.13) as expected from the relationship given in eq. (C.5). The asymmetry is near zero at the shorter wavelengths where the circular polarization is $\approx 50 \%$ (scaled), and it increases with increasing wavelength to its maximun in a manner similar to the circular polarization curve. 


\section{Bibliography}

[1] J. Kessler, Polarized Electrons (Springer, Berlin, 1985), 2nd ed.

[2] W.E. Spicer, Appl. Phys. 12, 115 (1977).

[3] S.F. Alvarado et al, Z. Phys. B 44, 259 (1981).

[4] T. Maruyama et al, Appl. Phys. Lett. 55, 1686 (1989).

[5] A.V. Aleksandrov et al, Phys. Rev. E 51, 1449 (1995).

[6] R. Prepost, in Proceedings of the Twenty First SLAC Summer Institute on Particle Physics, edited by L. DePorcel and C. Dunwoodie (SLAC Report 444, Stanford, 1994), p. 57.

[7] D.T. Pierce and R.J. Celotta, in Optical Orientation, edited by F. Mejer and B.P. Zakharchenya (North-Holland, Amsterdam, 1984), p. 259.

[8] P. Zurcher and F. Meier, J. Appl. Phys. 50, 3687 (1979).

[9] M.I. Dyakonov and V.I. Perel, in Optical Orientation edited by F. Meier and B.P. Zakharchenya (North-Holland, Amsterdam, 1984), p. 11.

[10] P. Zorabedian, Ph.D. Thesis, Stanford University, 1982.

[11] 3.H. van der Merwe, J. Appl. Phys. 34, 123 (1962).

[12] J.W. Matthews and A.E. Blakeslee, J. Cyyst. Growth, 27, 118 (1974).

[13] J.C. Bean et al, J. Vac. Sci. Technol. A 2, 436 (1984).

[14] P.J. Orders and B.F. Usher, Appl. Phys. Lett. 50, 980 (1987).

[15] R. People and J.C. Bean, Appl..Phys. Lett. 47, 322 (1985).

[16] T. Maruyama et al, Phys. Rev. Lett. 66, 2376 (1991).

[17] T. Nakanishi et al; Phys. Lett. A 158, 345 (1991).

[18] G.E. Pikus and G.L. Bir, Sov. Phys--Solid State 1, 1502 (1959). 
[19] See for example, C. Kittel, Quantum Theory of Solids (John Wiley and Sons, New York, 1987), 2nd ed.

[20] M.I. Dyakonov and V.I. Perel, Soviet Phys. JETP 33, 1053 (1971).

[21] E.O. Kane, J. Phys. Chem. Solids 1, 249 (1957).

[22] P.-O. Löwdin, J. Chem. Phys. 19, 1396 (1951).

[23] See for example, A.L. Mears and R.A. Stradling, J. Phys C 4, L22 (1971); J.M. Chamberlain and R.A. Strading, Solid State Commun., 7, 1275 (1969); M.S. Skolnick et al, J. Phys. C 9, 2809 (1976).

[24] H. Ehrenreich, J. Appl. Phys. 32 Suppl., 2155 (1961).

[25] Semiconductors, New Series III/17a of Landolt-Börnstein, edited by 0 . Madelung (Springer-Verlag, New York, 1982).

[26] G. Lasher and F. Stern, Phys. Rev. 133, A553 (1964).

[27] W. Van Roosbroeck and W. Shockley, Phys. Rev. 94, 1558 (1954).

[28] G.E. Pikus and A.N. Titkov, in Optical Orientation, edited by F. Meier and B.P. Zakbarchenya (North-Holland, Amsterdam, 1984), p. 73.

[29] G. Fishman and G. Lampel, Phys. Rev. B 16, 820 (1977).

[30] G.L. Bir, A.G. Aronov and G.E. Pikus, Sov. Phys,-JETP 42, 705 (1976).

[31] Spire Corposation, Bedford, MA 01730.

[32] S.M. Vernon et al, IEEE Photovoltaic Specialist's Conf. 19, 109 (1987).

[33] M.S. Abrahams et al, J. Mat. Sci. 4, 223 (1969).

[34] B. Schwartz et al, J. Electrochem. Soc. 123, 1089 (1976).

[35] T.M. Kerr, D.C. Peacock and C.E.C. Wood, J. Appl. Phys. 63, 1494 (1988).

[36] G. Duggan and G.B. Scott, J. Appl. Phys. 52, 407 (1981).

[37] R.A. Gottscho et al, J. Appl. Phys. 68, 460 (1990).

[38] D.J. Olego, R. Schachter and J.A. Baumann, Appl. Phys. Lett. 45, 1127 (1984).

[39] S.A. Chambers and V.S. Sundram, Appl. Phys. Lett. 57, 2342 (1990). 
[40] X.Y. Hou et al, Appl. Phys. Lett. 60, 2252 (1992).

[41] B.J. Skromme et al, Appl. Phys. Lett. 51, 2022 (1987).

[42] E.J. Thrush, J. Phys E 7, 493 (1974).

[43] C. Juang, K.J. Kuhn and R.B. Darling, J. Vac. Sci. Technol. B 8, 1122 (1990).

[44] C.L.H. Ashby and R.M. Biefeld, Appl. Phys. Lett. 47, 62 (1985). .

[45] R.M. Osgood Jr. et al, Appl. Phys. Lett. 40, 391 (1982).

[46] D.V. Podlesnik et of, Appl. Phys. Lett. 43, 1083 (1983).

[47] D. Moutonoet, Materials Lett. 6, 34 (1987).

[48] F. Kuhn-Kuhnenfeld, J. Electrochem. Soc. 119, 1063 (1972).

[49] Spraylat Corporation, Los Angeles, CA 90016.

[50] Acheson Colloids, Port Huron, MI 48060.

[51] Omega Engineering, Inc., Stamford, CT 06906.

[52] Spectra Physics - Laser Products Division, Mountain View, CA 94039.

[53] Instrument SA, Inc. - JY Optical Systems Division, Metuchen, NJ O8840.

[54] Princeton Instruments, Inc., Trenton, NJ 08619.

[55] J.D. Jackson, Classical Electrodynamics (John Wiley and Sons, New York, 1975), 2nd ed.

[56] Harold P. Klug and Leroy E. Alexander, X-Ray Diffraction Procedures (John Wiley and Sons, New York, 1954).

[57] Philips Analytical X-Ray B.V., Almelo, the Netherlands.

[58] J. Hornstra and W.J. Bartels, J. Cryst. Growth 44, 513 (1978).

[59] S. Adachi, J. Appl. Phys. 53, 8775 (1982).

[60] H.C. Casey, Jr. and M.B. Panish, Heterostructure Lasers, Part B (Academic, New York, 1978).

[61] R. Prepost and T. Maruyama, Annu. Rev. Nucl. Part. Sci. 45, 41 (1995).

[62] H.C. Casey, Jr. and F. Stern, J. Appl. Phys. 47, 631 (1976). 
[63] J.I. Pankove, Phys. Rev. 140, A2059 (1965).

[64] B.I. Halperin and M. Lax, Phys. Rev. 148, 722 (1966).

[65] E.O. Kane, Phys. Rev. 131, 79 (1963).

[66] C.K. Sinclais et al, in Proceedings of the Argonne Symposium on High Energy Physics with Polarized Beams and Targets, edited by M.L. Marshak (American Institute of Physics, New York, 1976), p.424.

[67] G. Mulhollan, in Proceedings of the Workshop on Photocathodes for Polarized Electron Sources for Accelerators, edited by M. Chatwell, J. Clendenin, T. Maruyama, and D. Schultz (SLAC Report 432, Stanford, 1994), p. 211.

[68] Y.P. Varshni, Physica 34, 149 (1967).

[69] D. Olego and M. Cardona, Phys. Rev. B 22, 886 (1980).

[70] G.L. Bir and G.E. Pikus, Symmetry and Strain-Induced Effects in Semiconductors (John Wiley and Sons, New York, 1974).

[71] J.S. Blakemore, Semiconductor Statistics, Vol. 3 of International Series of Monographs on Semiconductors, edited by H.K. Henisch (Pergamon Press, New York, 1962).

[72] See for example, F.H. Pollak et al, Phys. Rev. Lett. 16, 942 (1966); F.H. Pollak et al, Phys. Rev. 172, 816 (1968); I. Balslev, Phys. Rev. 177, 1173 (1969).

[73] D.E. Aspnes and A.A. Studna, Phys. Rev. B 27, 985 (1983).

[74] G.B. Scott, G. Duggan and P. Dawson, J. Appl. Phys. 52, 6888 (1981).

[75] See for example, W.E. Spicer et al, J. Vac. Sci. Technol. 16, 1422 (1979); T.E. Kazior, J. Lagowski and H.C. Gatos, J. Appl. Phys. 54, 2533 (1983).

[76] P. Pfeffer and 1. Gorczyca, Solid State Commun. 51, 179 (1984).

[77] D.Z. Garbuzov et al, Sovjet Physics - Solja State 14, 1481 (1972).

[78] R. Aliey et al, Nucl. Instr. and Methods in Physics Reseasch A 365, 1 (1995).

[79] R.A. Mair, 1995, unpublished data.

[80] T. Omori et al, int. J. Mod. Phys. A (Proc, Suppl) 2A, 157 (1993).

[8I] W. Bi - University of Californja at San Diego, 1995, private communication. 
[82] See for exareple, M.S. Abrahans et al, Appl. Phys. Lett. 21, 185 (1972); K.L. Kavanagh et af, J. Appl. Phys. 64, 4843 (1988); M. Grundmann et al, J. Vac. Sci. Technol. B8, 751 (1990); A. Benstada et al, J. Crystal Growth 130, 433 (1993).

[83] R.A. Majr et al, Phys. Lett. A 212, 231 (1996).

[84] P.F. Fewster, Appl. Surf. Sci. 50, 9 (1991). 